Prepared for the U.S. Department of Energy under Contract DE-AC05-76RL01830

\title{
Analytical Data Report of Grab Samples Collected From BP-5 Operable Unit O Well (C5852)
}

Michael Lindberg

September 2008

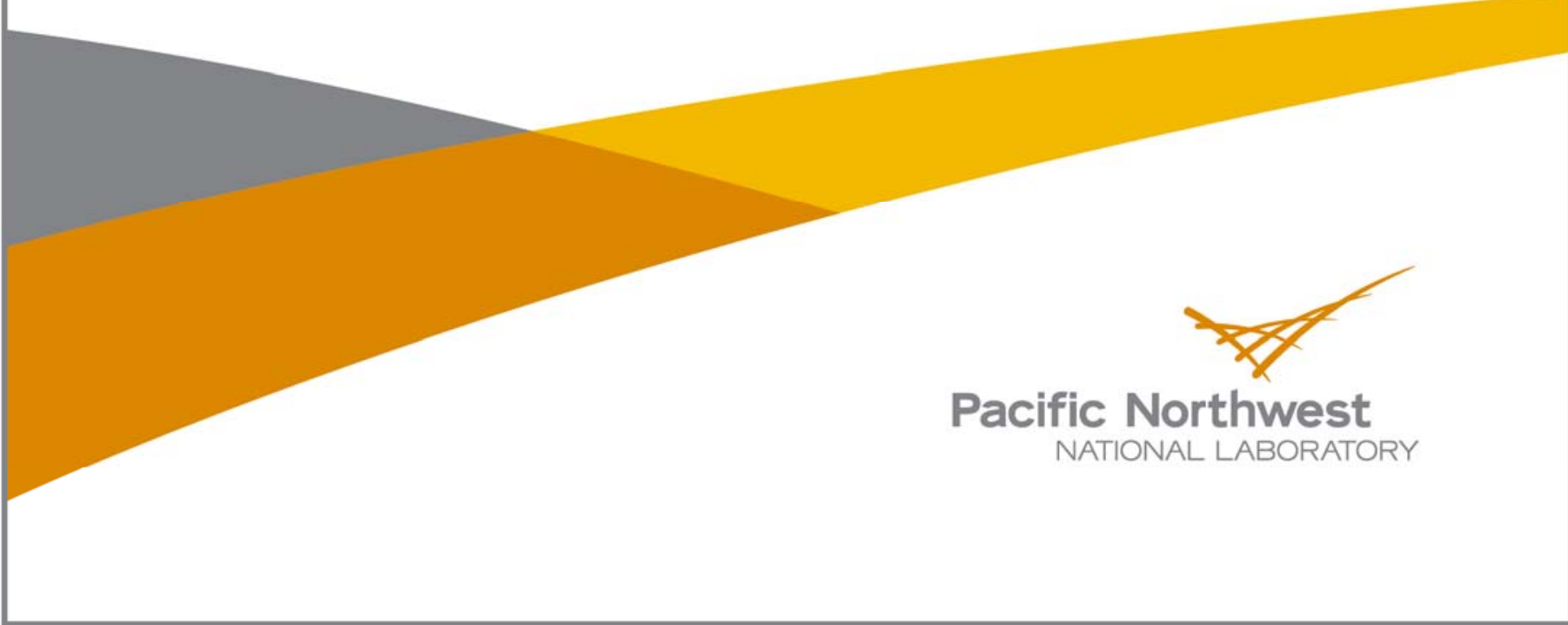


08/28/08 15:40

To: Dana Widrig

From: Michael J. Lindberg

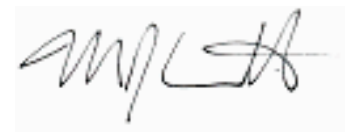

Environmental Sciences Laboratory

Energy and Environment Directorate, Pacific Northwest National Laboratory

Subject: Analytical Data Report of Grab Samples Collected From BP-5 Operable Unit O Well (C5852), Sample Delivery Group ESL070005, SAF Number F07-069

This letter contains the following information for sample delivery group ESL070005

- Cover Sheet

- Narrative

- Analytical Results

- Quality Control

- Geologic Photos

- Chain of Custodies 


\section{Introduction}

Between October 12, 2007 and October 28, 2007 soil cores and grab samples were received from BP-5 Operable Unit O Well (C5852) for geochemical studies.

\section{Analytical Results/Methodology}

The analyses for this project were performed at the 325 building located in the 300 Area of the Hanford Site. The analyses were performed according to Pacific Northwest National Laboratory (PNNL) approved procedures and/or nationally recognized test procedures. The data sets include the sample identification numbers, analytical results, estimated quantification limits (EQL), and quality control data.

\section{Quality Control}

The preparatory and analytical quality control requirements, calibration requirements, acceptance criteria, and failure actions are defined in the on-line QA plan “Conducting Analytical Work in Support of Regulatory Programs” (CAW). This QA plan implements the Hanford Analytical Services Quality Assurance Requirements Documents (HASQARD) for PNNL.

\section{Definitions}

Dup Duplicate

RPD Relative Percent Difference

\section{Sample Receipt}

Samples were received with a chain of custody (COC) and were analyzed according to the sample identification numbers supplied by the client. All Samples were refrigerated upon receipt until prepared for analysis.

All samples were received with custody seals intact unless noted in the Case Narrative.

\section{Holding Times}

Holding time is defined as the time from sample preparation to the time of analyses. The prescribed holding times were met for all analytes unless noted in the Case Narrative.

\section{Analytical Results}

All reported analytical results meet the requirements of the CAW or client specified SOW unless noted in the case narrative. 


\section{Case Narrative Report}

Hold time:

No discrepancies noted.

Preparation Blank (PB):

No discrepancies noted.

\section{Duplicate (DUP):}

No discrepancies noted.

\section{Laboratory control samples (LCS):}

No discrepancies noted.

Post spike (PS) and post spike duplicate (PSD):

Post-Spike Recovery for Sodium (126\%) was outside acceptance limits (75-125) in 8E01004-PS1 for ICP-OES Vadose-WE

The concentration of the analyte in the original sample is greater than $1000 \mathrm{ppm}$. Therefore, spikes are not required per HASQARD.

Matrix spike (MS) and matrix spike duplicate (MSD):

Not Applicable

\section{Other QC Criteria:}

No discrepancies noted.

\section{DISCLAIMER}

This report was prepared as an account of work sponsored by an agency of the United States Government. Neither the United States Government nor any agency thereof, nor Battelle Memorial Institute, nor any of their employees, makes any warranty, express or implied, or assumes any legal liability or responsibility for the accuracy, completeness, or usefulness of any information, apparatus, product, or process disclosed, or represents that its use would not infringe privately owned rights. Reference herein to any specific commercial product, process, or service by trade name, trademark, manufacturer, or otherwise does not necessarily constitute or imply its endorsement, recommendation, or favoring by the United States Government or any agency thereof, or Battelle Memorial Institute. The views and opinions of authors expressed herein do not necessarily state or reflect those of the United States Government or any agency thereof. 


\section{SAMPLES INCLUDED IN THIS REPORT}

\section{BP 5 OU, C5852 O-Well VZ}

$\begin{array}{lll}\text { HEIS No. } & \begin{array}{l}\text { Laboratory ID } \\ 0802019-01\end{array} & \text { Matrix } \\ \text { B1PMY9 } & 0802019-03 & \text { SOIL } \\ \text { B1PN01 } & 0802020-01 & \text { SOIL } \\ \text { B1PN03 } & 0802020-03 & \text { SOIL } \\ \text { B1PN05 } & 0802020-05 & \text { SOIL } \\ \text { B1PN07 } & 0802020-09 & \text { SOIL } \\ \text { B1PN09 } & 0802020-11 & \text { SOIL } \\ \text { B1PN11 } & 0802020-17 & \text { SOIL } \\ \text { B1R100 } & 0802023-09 & \text { SOIL } \\ \text { B1PN17 } & 0802023-10 & \text { SOIL } \\ \text { B1PN18 } & 0802023-19 & \text { SOIL } \\ \text { B1PN25 } & 0802023-20 & \text { SOIL } \\ \text { B1PN26 } & & \end{array}$

\begin{tabular}{llll} 
Date Collected & \multicolumn{2}{c}{ Date Received } \\
10/11/07 $13: 50$ & 10/12/07 $09: 00$ \\
10/11/07 & $14: 32$ & $10 / 12 / 07$ & $09: 00$ \\
10/12/07 & $07: 48$ & $10 / 17 / 07$ & $08: 55$ \\
10/12/07 & $09: 16$ & $10 / 17 / 07$ & $08: 55$ \\
10/12/07 & $10: 33$ & $10 / 17 / 07$ & $08: 55$ \\
10/12/07 & $14: 35$ & $10 / 17 / 07$ & $08: 55$ \\
10/15/07 & $08: 10$ & $10 / 17 / 07$ & $08: 55$ \\
10/16/07 & $09: 42$ & $10 / 17 / 07$ & $08: 55$ \\
10/18/07 & $14: 20$ & $10 / 24 / 07$ & $08: 30$ \\
10/19/07 & $14: 00$ & $10 / 24 / 07$ & $08: 30$ \\
10/23/07 & $14: 05$ & $10 / 23 / 07$ & $15: 00$ \\
10/24/07 & $12: 50$ & $10 / 25 / 07$ & $12: 50$
\end{tabular}


The following analyses were performed on the following samples included in this report:

Metals 1:1 DI Water Extract by ICPMS

1:1 DI Water Extract

Alkalinity, Titrimetic ( $\mathrm{pH} 4.5)$

Anions By Ion Chromatography

geological description

Metals 1:1 Water Extract by ICPOES

Moisture Content

pH of Waters By Electrode

Specific Conductance

Tc_U 1:1 DI Water Extract by ICPMS

\section{SAMPLES ANALYZED IN THIS REPORT}

$\begin{array}{lll}\text { HEIS No. } & \begin{array}{l}\text { Laboratory ID } \\ 0802019-01\end{array} & \begin{array}{l}\text { Matrix } \\ \text { SOIL }\end{array} \\ \text { B1PMY9 } & 0802019-03 & \text { SOIL } \\ \text { B1PN01 } & 0802020-01 & \text { SOIL } \\ \text { B1PN03 } & 0802020-03 & \text { SOIL } \\ \text { B1PN05 } & 0802020-05 & \text { SOIL } \\ \text { B1PN07 } & 0802020-09 & \text { SOIL } \\ \text { B1PN09 } & 0802020-11 & \text { SOIL } \\ \text { B1PN11 } & 0802020-17 & \text { SOIL } \\ \text { B1R100 } & 0802023-09 & \text { SOIL } \\ \text { B1PN17 } & 0802023-10 & \text { SOIL } \\ \text { B1PN18 } & 0802023-19 & \text { SOIL } \\ \text { B1PN25 } & 0802023-20 & \text { SOIL } \\ \text { B1PN26 } & & \end{array}$

$\begin{array}{ll}\text { Date Collected } \\ \text { 10/11/07 } & 13: 50 \\ \text { 10/11/07 } & 14: 32 \\ \text { 10/12/07 } & 07: 48 \\ \text { 10/12/07 } & 09: 16 \\ 10 / 12 / 07 & 10: 33 \\ 10 / 12 / 07 & 14: 35 \\ 10 / 15 / 07 & 08: 10 \\ 10 / 16 / 07 & 09: 42 \\ 10 / 18 / 07 & 14: 20 \\ 10 / 19 / 07 & 14: 00 \\ 10 / 23 / 07 & 14: 05 \\ 10 / 24 / 07 & 12: 50\end{array}$

Date Received 10/12/07 09:00 10/12/07 09:00 10/17/07 08:55 10/17/07 08:55 10/17/07 08:55 10/17/07 08:55 10/17/07 08:55 10/17/07 08:55 10/24/07 08:30 10/24/07 08:30 10/23/07 15:00 10/25/07 12:50 


\section{Wet Chemistry}

\begin{tabular}{|c|c|c|c|c|c|}
\hline $\begin{array}{l}\text { Alkalinity } \\
\text { Lab ID }\end{array}$ & $\begin{array}{c}\text { CaCO3 } \\
\text { HEIS No. }\end{array}$ & $\begin{array}{l}\text { Methoc } \\
\text { Results }\end{array}$ & $\begin{array}{c}\text { OB } \\
\text { EQL }\end{array}$ & Analyzed & Batch \\
\hline 0802019-01 & В1PMY9 & 4.50E1 & 2.49E1 & $11 / 29 / 07$ & 8D07003 \\
\hline 0802019-03 & B1PN01 & $4.18 \mathrm{E} 1$ & 2.35E1 & $4 / 29 / 08$ & 8D07003 \\
\hline 0802020-01 & B1PN03 & 3.83E1 & 2.42E1 & 11/29/07 & 8D07003 \\
\hline 0802020-03 & B1PN05 & 5.03E1 & 2.55E1 & $11 / 29 / 07$ & 8D07003 \\
\hline 0802020-05 & B1PN07 & 3.59E1 & 2.36E1 & $11 / 29 / 07$ & 8D07003 \\
\hline 0802020-09 & B1PN09 & 5.13E1 & 2.37E1 & $11 / 29 / 07$ & 8D07003 \\
\hline 0802020-11 & B1PN11 & 4.85E1 & 2.42E1 & 11/29/07 & 8D07003 \\
\hline 0802020-17 & B1R100 & $5.10 \mathrm{E} 1$ & 2.36E1 & 11/29/07 & 8D07003 \\
\hline 0802023-09 & B1PN17 & 4.34E1 & 2.35E1 & $11 / 29 / 07$ & 8D07003 \\
\hline 0802023-10 & B1PN18 & 4.88E1 & 2.36E1 & $11 / 29 / 07$ & 8D07003 \\
\hline 0802023-19 & B1PN25 & 4.42E1 & $2.40 \mathrm{E} 1$ & 11/29/07 & 8D07003 \\
\hline 0802023-20 & B1PN26 & 4.44E1 & 2.41E1 & $11 / 29 / 07$ & 8D07003 \\
\hline
\end{tabular}




\section{Wet Chemistry}

\begin{tabular}{|c|c|c|c|c|c|}
\hline \multicolumn{6}{|c|}{ Specific Conductance (EC) (mS/cm) by EPA 120.1} \\
\hline Lab ID & HEIS No. & Results & EQL & Analyzed & Batch \\
\hline 0802019-01 & B1PMY9 & $2.71 \mathrm{E}-1$ & $1.00 \mathrm{E}-2$ & 11/28/07 & 8D07003 \\
\hline 0802019-03 & B1PN01 & $3.22 \mathrm{E}-1$ & $1.00 \mathrm{E}-2$ & $11 / 28 / 07$ & 8D07003 \\
\hline 0802020-01 & B1PN03 & $2.41 \mathrm{E}-1$ & $1.00 \mathrm{E}-2$ & $11 / 28 / 07$ & 8D07003 \\
\hline 0802020-03 & B1PN05 & $2.11 \mathrm{E}-1$ & $1.00 \mathrm{E}-2$ & $11 / 28 / 07$ & 8D07003 \\
\hline 0802020-05 & B1PN07 & $2.21 \mathrm{E}-1$ & $1.00 \mathrm{E}-2$ & $11 / 28 / 07$ & 8D07003 \\
\hline 0802020-09 & B1PN09 & 2.46E-1 & $1.00 \mathrm{E}-2$ & $11 / 28 / 07$ & 8D07003 \\
\hline 0802020-11 & B1PN11 & $2.87 \mathrm{E}-1$ & $1.00 \mathrm{E}-2$ & $11 / 28 / 07$ & 8D07003 \\
\hline 0802020-17 & B1R100 & $1.37 \mathrm{E}-1$ & $1.00 \mathrm{E}-2$ & $11 / 28 / 07$ & 8D07003 \\
\hline 0802023-09 & B1PN17 & $1.07 \mathrm{E}-1$ & $1.00 \mathrm{E}-2$ & $11 / 28 / 07$ & 8D07003 \\
\hline 0802023-10 & B1PN18 & $1.06 \mathrm{E}-1$ & $1.00 \mathrm{E}-2$ & 11/28/07 & 8D07003 \\
\hline 0802023-19 & B1PN25 & $1.05 \mathrm{E}-1$ & $1.00 \mathrm{E}-2$ & 11/28/07 & 8D07003 \\
\hline 0802023-20 & B1PN26 & $1.02 \mathrm{E}-1$ & $1.00 \mathrm{E}-2$ & $11 / 28 / 07$ & 8D07003 \\
\hline
\end{tabular}




\section{Wet Chemistry}

Moisture Content (\% by Weight) by AGG-WC-001

\begin{tabular}{llcccc} 
Lab ID & HEIS No. & Results & EQL & Analyzed & Batch \\
\hline $0802019-01$ & B1PMY9 & $4.49 \mathrm{E} 0$ & N/A & $4 / 21 / 08$ & $8 \mathrm{D} 08004$ \\
$0802019-03$ & B1PN01 & $4.33 \mathrm{E} 0$ & N/A & $4 / 21 / 08$ & $8 \mathrm{D} 08004$ \\
$0802020-01$ & B1PN03 & $4.09 \mathrm{E} 0$ & N/A & $4 / 21 / 08$ & $8 \mathrm{D} 08004$ \\
$0802020-03$ & B1PN05 & $2.74 \mathrm{E} 0$ & N/A & $4 / 21 / 08$ & $8 \mathrm{D} 08004$ \\
$0802020-05$ & B1PN07 & $3.23 \mathrm{E} 0$ & N/A & $4 / 21 / 08$ & $8 \mathrm{D} 08004$ \\
$0802020-09$ & B1PN09 & $2.42 \mathrm{E} 0$ & N/A & $4 / 21 / 08$ & $8 \mathrm{D} 08004$ \\
$0802020-11$ & B1PN11 & $4.56 \mathrm{E} 0$ & N/A & $4 / 21 / 08$ & $8 \mathrm{D} 08004$ \\
$0802020-17$ & B1R100 & $9.19 \mathrm{E} 0$ & N/A & $4 / 21 / 08$ & $8 \mathrm{D} 08004$ \\
$0802023-09$ & B1PN17 & $7.43 \mathrm{E} 0$ & N/A & $4 / 21 / 08$ & $8 \mathrm{D} 08004$ \\
$0802023-10$ & B1PN18 & $8.44 \mathrm{E} 0$ & N/A & $4 / 21 / 08$ & $8 \mathrm{D} 08004$ \\
$0802023-19$ & B1PN25 & $6.59 \mathrm{E} 0$ & N/A & $4 / 21 / 08$ & $8 \mathrm{D} 08004$ \\
$0802023-20$ & B1PN26 & $6.81 \mathrm{E} 0$ & N/A & 8D08004 \\
\hline
\end{tabular}




\section{Wet Chemistry}

pH (pH Units) by AGG-pH-001

\begin{tabular}{llcccc|} 
Lab ID & HEIS No. & Results & EQL & Analyzed & Batch \\
\hline $0802019-01$ & B1PMY9 & $7.16 \mathrm{E} 0$ & N/A & $11 / 28 / 07$ & $8 \mathrm{D} 30002$ \\
$0802019-03$ & B1PN01 & $7.23 \mathrm{E} 0$ & N/A & $12 / 05 / 07$ & $8 \mathrm{D} 30002$ \\
$0802020-01$ & B1PN03 & $7.45 \mathrm{E} 0$ & N/A & $12 / 05 / 07$ & $8 \mathrm{D} 30002$ \\
$0802020-03$ & B1PN05 & $7.56 \mathrm{E} 0$ & N/A & $11 / 28 / 07$ & $8 \mathrm{D} 30002$ \\
$0802020-05$ & B1PN07 & $7.09 \mathrm{E} 0$ & N/A & $11 / 28 / 07$ & $8 \mathrm{D} 30002$ \\
$0802020-09$ & B1PN09 & $7.71 \mathrm{E} 0$ & N/A & $11 / 28 / 07$ & $8 \mathrm{D} 30002$ \\
$0802020-11$ & B1PN11 & $7.47 \mathrm{E} 0$ & N/A & $11 / 28 / 07$ & $8 \mathrm{D} 30002$ \\
$0802020-17$ & B1R100 & $7.60 \mathrm{E} 0$ & N/A & $11 / 28 / 07$ & $8 \mathrm{D} 30002$ \\
$0802023-09$ & B1PN17 & $7.44 \mathrm{E} 0$ & N/A & $11 / 28 / 07$ & $8 \mathrm{D} 30002$ \\
$0802023-10$ & B1PN18 & $7.28 \mathrm{E} 0$ & N/A & $11 / 28 / 07$ & 8D30002 \\
$0802023-19$ & B1PN25 & $7.47 \mathrm{E} 0$ & N/A & $11 / 28 / 07$ & 8D30002 \\
$0802023-20$ & B1PN26 & $7.39 \mathrm{E} 0$ & N/A & 8D30002 \\
\hline
\end{tabular}




\section{Anions by Ion Chromatography}

\begin{tabular}{|c|c|c|c|c|c|c|c|}
\hline CAS \# & Analyte & Results & Units & EQL & Analyzed & Batch & Method \\
\hline HEIS No. & B1PMY9 & \multicolumn{3}{|c|}{ Lab ID: $\quad$ 0802019-01 } & & & \\
\hline $16984-48-8$ & Fluoride & $<2.12 \mathrm{E} 0$ & ug/g dry & 2.12E0 & $11 / 28 / 07$ & 8D25002 & AGG-IC-001 \\
\hline $14797-65-0$ & Nitrite & $<1.06 \mathrm{E} 1$ & ug/g dry & $1.06 \mathrm{E} 1$ & $11 / 28 / 07$ & 8D25002 & AGG-IC-001 \\
\hline $14797-55-8$ & Nitrate & 8.05E0 & ug/g dry & $1.06 \mathrm{E} 0$ & $12 / 05 / 07$ & 8D25002 & AGG-IC-001 \\
\hline 14808-79-8 & Sulfate & $6.76 \mathrm{E} 1$ & ug/g dry & $1.59 \mathrm{E} 1$ & $11 / 28 / 07$ & 8D25002 & AGG-IC-001 \\
\hline 14265-44-2 & Phosphate & $<1.59 \mathrm{E} 1$ & ug/g dry & $1.59 \mathrm{E} 1$ & $11 / 28 / 07$ & 8D25002 & AGG-IC-001 \\
\hline HEIS No. & B1PN01 & \multicolumn{3}{|c|}{ Lab ID: $\quad$ 0802019-03 } & & & \\
\hline $16984-48-8$ & Fluoride & $<2.00 \mathrm{E} 0$ & ug/g dry & $2.00 \mathrm{E} 0$ & $11 / 28 / 07$ & 8D25002 & AGG-IC-001 \\
\hline $14797-65-0$ & Nitrite & $<1.00 \mathrm{E} 1$ & ug/g dry & $1.00 \mathrm{E} 1$ & $11 / 28 / 07$ & 8D25002 & AGG-IC-001 \\
\hline $14797-55-8$ & Nitrate & 9.45E0 & ug/g dry & $1.00 \mathrm{E} 0$ & $12 / 05 / 07$ & 8D25002 & AGG-IC-001 \\
\hline 14808-79-8 & Sulfate & $8.41 \mathrm{E} 1$ & ug/g dry & $1.50 \mathrm{E} 1$ & $11 / 28 / 07$ & 8D25002 & AGG-IC-001 \\
\hline 14265-44-2 & Phosphate & $<1.50 \mathrm{E} 1$ & ug/g dry & $1.50 \mathrm{E} 1$ & $11 / 28 / 07$ & 8D25002 & AGG-IC-001 \\
\hline HEIS No. & B1PN03 & \multicolumn{3}{|c|}{$\begin{array}{ll}\text { Lab ID: } & \text { 0802020-01 }\end{array}$} & & & \\
\hline $16984-48-8$ & Fluoride & $<2.06 \mathrm{E} 0$ & ug/g dry & 2.06E0 & $11 / 28 / 07$ & 8D25002 & AGG-IC-001 \\
\hline $14797-65-0$ & Nitrite & $<1.03 \mathrm{E} 1$ & ug/g dry & 1.03E1 & $11 / 28 / 07$ & 8D25002 & AGG-IC-001 \\
\hline 14797-55-8 & Nitrate & $7.50 \mathrm{E} 0$ & ug/g dry & $1.03 \mathrm{E} 0$ & $12 / 05 / 07$ & 8D25002 & AGG-IC-001 \\
\hline $14808-79-8$ & Sulfate & 5.92E1 & ug/g dry & $1.54 \mathrm{E} 1$ & $11 / 28 / 07$ & 8D25002 & AGG-IC-001 \\
\hline 14265-44-2 & Phosphate & $<1.54 \mathrm{E} 1$ & ug/g dry & $1.54 \mathrm{E} 1$ & $11 / 28 / 07$ & 8D25002 & AGG-IC-001 \\
\hline HEIS No. & B1PN05 & \multicolumn{3}{|c|}{ Lab ID: $\quad$ 0802020-03 } & & & \\
\hline $16984-48-8$ & Fluoride & $<2.17 \mathrm{E} 0$ & ug/g dry & 2.17E0 & $11 / 28 / 07$ & 8D25002 & AGG-IC-001 \\
\hline $14797-65-0$ & Nitrite & $<1.09 \mathrm{E} 1$ & ug/g dry & $1.09 \mathrm{E} 1$ & $11 / 28 / 07$ & 8D25002 & AGG-IC-001 \\
\hline $14797-55-8$ & Nitrate & 5.58E0 & ug/g dry & $1.09 \mathrm{E} 0$ & $12 / 05 / 07$ & 8D25002 & AGG-IC-001 \\
\hline $14808-79-8$ & Sulfate & 4.82E1 & ug/g dry & 1.63E1 & $11 / 28 / 07$ & 8D25002 & AGG-IC-001 \\
\hline $14265-44-2$ & Phosphate & $<1.63 \mathrm{E} 1$ & ug/g dry & $1.63 \mathrm{E} 1$ & $11 / 28 / 07$ & 8D25002 & AGG-IC-001 \\
\hline HEIS No. & B1PN07 & \multicolumn{3}{|c|}{ Lab ID: $\quad$ 0802020-05 } & & & \\
\hline 16984-48-8 & Fluoride & $<2.01 \mathrm{E} 0$ & ug/g dry & 2.01E0 & $11 / 28 / 07$ & 8D25002 & AGG-IC-001 \\
\hline $14797-65-0$ & Nitrite & $<1.00 \mathrm{E} 1$ & ug/g dry & $1.00 \mathrm{E} 1$ & $11 / 28 / 07$ & 8D25002 & AGG-IC-001 \\
\hline $14797-55-8$ & Nitrate & 5.72E0 & ug/g dry & $1.00 \mathrm{E} 0$ & $12 / 05 / 07$ & 8D25002 & AGG-IC-001 \\
\hline 14808-79-8 & Sulfate & $4.84 \mathrm{E} 1$ & ug/g dry & $1.51 \mathrm{E} 1$ & $11 / 28 / 07$ & 8D25002 & AGG-IC-001 \\
\hline $14265-44-2$ & Phosphate & $<1.51 \mathrm{E} 1$ & ug/g dry & $1.51 \mathrm{E} 1$ & $11 / 28 / 07$ & 8D25002 & AGG-IC-001 \\
\hline HEIS No. & B1PN09 & \multicolumn{3}{|c|}{$\begin{array}{ll}\text { Lab ID: } & \text { 0802020-09 }\end{array}$} & & & \\
\hline $16984-48-8$ & Fluoride & $<2.02 \mathrm{E} 0$ & ug/g dry & $2.02 \mathrm{E} 0$ & $11 / 28 / 07$ & 8D25002 & AGG-IC-001 \\
\hline $14797-65-0$ & Nitrite & $<1.01 \mathrm{E} 1$ & ug/g dry & $1.01 \mathrm{E} 1$ & $11 / 28 / 07$ & 8D25002 & AGG-IC-001 \\
\hline $14797-55-8$ & Nitrate & 4.94E0 & ug/g dry & $1.01 \mathrm{E} 0$ & $12 / 05 / 07$ & 8D25002 & AGG-IC-001 \\
\hline 14808-79-8 & Sulfate & 4.79E1 & ug/g dry & $1.51 \mathrm{E} 1$ & $11 / 28 / 07$ & 8D25002 & AGG-IC-001 \\
\hline 14265-44-2 & Phosphate & $<1.51 \mathrm{E} 1$ & ug/g dry & $1.51 \mathrm{E} 1$ & $11 / 28 / 07$ & 8D25002 & AGG-IC-001 \\
\hline HEIS No. & B1PN11 & \multicolumn{3}{|c|}{ Lab ID: $\quad$ 0802020-11 } & & & \\
\hline $16984-48-8$ & Fluoride & $<2.06 \mathrm{E} 0$ & ug/g dry & 2.06E0 & $11 / 28 / 07$ & 8D25002 & AGG-IC-001 \\
\hline $14797-65-0$ & Nitrite & $<1.03 \mathrm{E} 1$ & ug/g dry & $1.03 \mathrm{E} 1$ & $11 / 28 / 07$ & 8D25002 & AGG-IC-001 \\
\hline $14797-55-8$ & Nitrate & 8.91E0 & ug/g dry & $1.03 \mathrm{E} 0$ & $12 / 05 / 07$ & 8D25002 & AGG-IC-001 \\
\hline 14808-79-8 & Sulfate & 7.09E1 & ug/g dry & $1.54 \mathrm{E} 1$ & $11 / 28 / 07$ & 8D25002 & AGG-IC-001 \\
\hline 14265-44-2 & Phosphate & $<1.54 \mathrm{E} 1$ & ug/g dry & $1.54 \mathrm{E} 1$ & $11 / 28 / 07$ & 8D25002 & AGG-IC-001 \\
\hline HEIS No. & B1R100 & \multicolumn{3}{|c|}{ Lab ID: $\quad$ 0802020-17 } & & & \\
\hline $16984-48-8$ & Fluoride & $<2.00 \mathrm{E} 0$ & ug/g dry & $2.00 \mathrm{E} 0$ & $11 / 28 / 07$ & 8D25002 & AGG-IC-001 \\
\hline $14797-65-0$ & Nitrite & $<1.00 \mathrm{E} 1$ & ug/g dry & $1.00 \mathrm{E} 1$ & $11 / 28 / 07$ & 8D25002 & AGG-IC-001 \\
\hline $14797-55-8$ & Nitrate & $<1.00 \mathrm{E} 1$ & ug/g dry & $1.00 \mathrm{E} 1$ & $11 / 28 / 07$ & 8D25002 & AGG-IC-001 \\
\hline $14808-79-8$ & Sulfate & $1.86 \mathrm{E} 1$ & ug/g dry & $1.50 \mathrm{E} 1$ & 11/28/07 & 8D25002 & AGG-IC-001 \\
\hline 14265-44-2 & Phosphate & $<1.50 \mathrm{E} 1$ & ug/g dry & $1.50 \mathrm{E} 1$ & $11 / 28 / 07$ & 8D25002 & AGG-IC-001 \\
\hline
\end{tabular}




\section{Anions by Ion Chromatography}

\begin{tabular}{|c|c|c|c|c|c|c|c|}
\hline CAS \# & Analyte & Results & Units & EQL & Analyzed & Batch & Method \\
\hline HEIS No. & B1PN17 & \multicolumn{3}{|c|}{ Lab ID: $\quad$ 0802023-09 } & & & \\
\hline 16984-48-8 & Fluoride & $<2.00 \mathrm{E} 0$ & ug/g dry & $2.00 \mathrm{E} 0$ & $11 / 28 / 07$ & 8D25002 & AGG-IC-001 \\
\hline 14797-65-0 & Nitrite & $<1.00 \mathrm{E} 1$ & ug/g dry & $1.00 \mathrm{E} 1$ & $11 / 28 / 07$ & 8D25002 & AGG-IC-001 \\
\hline 14797-55-8 & Nitrate & $<1.00 \mathrm{E} 0$ & ug/g dry & $1.00 \mathrm{E} 0$ & $12 / 05 / 07$ & 8D25002 & AGG-IC-001 \\
\hline 14808-79-8 & Sulfate & $<1.50 \mathrm{E} 1$ & ug/g dry & $1.50 \mathrm{E} 1$ & $11 / 28 / 07$ & 8D25002 & AGG-IC-001 \\
\hline $14265-44-2$ & Phosphate & $<1.50 \mathrm{E} 1$ & ug/g dry & $1.50 \mathrm{E} 1$ & $11 / 28 / 07$ & 8D25002 & AGG-IC-001 \\
\hline HEIS No. & B1PN18 & \multicolumn{3}{|c|}{ Lab ID: $\quad$ 0802023-10 } & & & \\
\hline $16984-48-8$ & Fluoride & $<2.01 \mathrm{E} 0$ & ug/g dry & $2.01 \mathrm{E} 0$ & 11/28/07 & 8D25002 & AGG-IC-001 \\
\hline 14797-65-0 & Nitrite & $<1.00 \mathrm{E} 1$ & ug/g dry & $1.00 \mathrm{E} 1$ & $11 / 28 / 07$ & 8D25002 & AGG-IC-001 \\
\hline 14797-55-8 & Nitrate & $1.58 \mathrm{E} 0$ & ug/g dry & $1.00 \mathrm{E} 0$ & $12 / 05 / 07$ & 8D25002 & AGG-IC-001 \\
\hline 14808-79-8 & Sulfate & $1.52 \mathrm{E} 1$ & ug/g dry & $1.51 \mathrm{E} 1$ & $11 / 28 / 07$ & 8D25002 & AGG-IC-001 \\
\hline $14265-44-2$ & Phosphate & $<1.51 \mathrm{E} 1$ & ug/g dry & $1.51 \mathrm{E} 1$ & $11 / 28 / 07$ & 8D25002 & AGG-IC-001 \\
\hline HEIS No. & B1PN25 & \multicolumn{3}{|c|}{ Lab ID: $\quad$ 0802023-19 } & & & \\
\hline 16984-48-8 & Fluoride & $<2.04 \mathrm{E} 0$ & ug/g dry & 2.04E0 & $11 / 28 / 07$ & 8D25002 & AGG-IC-001 \\
\hline $14797-65-0$ & Nitrite & $<1.02 \mathrm{E} 1$ & ug/g dry & $1.02 \mathrm{E} 1$ & $11 / 28 / 07$ & 8D25002 & AGG-IC-001 \\
\hline $14797-55-8$ & Nitrate & $<1.02 \mathrm{E} 0$ & ug/g dry & $1.02 \mathrm{E} 0$ & $12 / 05 / 07$ & 8D25002 & AGG-IC-001 \\
\hline 14808-79-8 & Sulfate & $<1.53 \mathrm{E} 1$ & ug/g dry & $1.53 \mathrm{E} 1$ & $11 / 28 / 07$ & 8D25002 & AGG-IC-001 \\
\hline $14265-44-2$ & Phosphate & $<1.53 \mathrm{E} 1$ & ug/g dry & $1.53 \mathrm{E} 1$ & $11 / 28 / 07$ & 8D25002 & AGG-IC-001 \\
\hline HEIS No. & B1PN26 & \multicolumn{3}{|c|}{ Lab ID: $\quad$ 0802023-20 } & & & \\
\hline 16984-48-8 & Fluoride & $<2.05 \mathrm{E} 0$ & ug/g dry & 2.05E0 & 11/29/07 & 8D25002 & AGG-IC-001 \\
\hline 14797-65-0 & Nitrite & $<1.02 \mathrm{E} 1$ & ug/g dry & $1.02 \mathrm{E} 1$ & 11/29/07 & 8D25002 & AGG-IC-001 \\
\hline $14797-55-8$ & Nitrate & $1.29 \mathrm{E} 0$ & ug/g dry & $1.02 \mathrm{E} 0$ & $12 / 05 / 07$ & 8D25002 & AGG-IC-001 \\
\hline 14808-79-8 & Sulfate & $<1.54 \mathrm{E} 1$ & ug/g dry & $1.54 \mathrm{E} 1$ & 11/29/07 & 8D25002 & AGG-IC-001 \\
\hline 14265-44-2 & Phosphate & $<1.54 \mathrm{E} 1$ & ug/g dry & $1.54 \mathrm{E} 1$ & 11/29/07 & 8D25002 & AGG-IC-001 \\
\hline
\end{tabular}


Total Metals by PNNL-AGG-ICP-AES/Water Extract

\begin{tabular}{|c|c|c|c|c|c|c|c|}
\hline CAS \# & Analyte & Results & Units & EQL & Analyzed & Batch & Method \\
\hline HEIS No. & B1PMY9 & \multicolumn{3}{|c|}{ Lab ID: $\quad$ 0802019-01 } & & & \\
\hline $7429-90-5$ & Aluminum & $<9.08 \mathrm{E}-2$ & ug/g dry & $9.08 \mathrm{E}-2$ & $12 / 05 / 07$ & 8E01004 & PNNL-AGG-ICP-AES \\
\hline $7440-70-2$ & Calcium & 2.02E1 & ug/g dry & $4.10 \mathrm{E}-1$ & $12 / 05 / 07$ & 8E01004 & PNNL-AGG-ICP-AES \\
\hline $7440-47-3$ & Chromium & $<3.52 \mathrm{E}-2$ & ug/g dry & $3.52 \mathrm{E}-2$ & $12 / 05 / 07$ & 8E01004 & PNNL-AGG-ICP-AES \\
\hline $7440-09-7$ & Potassium & 5.83E0 & ug/g dry & $2.46 \mathrm{E} 0$ & $12 / 05 / 07$ & 8E01004 & PNNL-AGG-ICP-AES \\
\hline 7439-95-4 & Magnesium & $4.90 \mathrm{E} 0$ & ug/g dry & 8.83E-2 & $12 / 05 / 07$ & 8E01004 & PNNL-AGG-ICP-AES \\
\hline 7439-96-5 & Manganese & $<1.81 \mathrm{E}-2$ & ug/g dry & $1.81 \mathrm{E}-2$ & $12 / 05 / 07$ & 8E01004 & PNNL-AGG-ICP-AES \\
\hline $7440-23-5$ & Sodium & 1.89E1 & ug/g dry & 7.08E-1 & $12 / 05 / 07$ & 8E01004 & PNNL-AGG-ICP-AES \\
\hline HEIS No. & B1PN01 & \multicolumn{3}{|c|}{ Lab ID: $\quad$ 0802019-03 } & & & \\
\hline $7429-90-5$ & Aluminum & $<8.58 \mathrm{E}-2$ & ug/g dry & $8.58 \mathrm{E}-2$ & $12 / 05 / 07$ & 8E01004 & PNNL-AGG-ICP-AES \\
\hline $7440-70-2$ & Calcium & 2.12E1 & ug/g dry & 3.87E-1 & $12 / 05 / 07$ & 8E01004 & PNNL-AGG-ICP-AES \\
\hline $7440-47-3$ & Chromium & $<3.33 \mathrm{E}-2$ & ug/g dry & 3.33E-2 & $12 / 05 / 07$ & 8E01004 & PNNL-AGG-ICP-AES \\
\hline 7440-09-7 & Potassium & 7.32Е0 & ug/g dry & 2.33Е0 & $12 / 05 / 07$ & 8E01004 & PNNL-AGG-ICP-AES \\
\hline 7439-95-4 & Magnesium & $5.11 \mathrm{E} 0$ & ug/g dry & $8.34 \mathrm{E}-2$ & $12 / 05 / 07$ & 8E01004 & PNNL-AGG-ICP-AES \\
\hline 7439-96-5 & Manganese & $<1.71 \mathrm{E}-2$ & ug/g dry & $1.71 \mathrm{E}-2$ & $12 / 05 / 07$ & 8E01004 & PNNL-AGG-ICP-AES \\
\hline $7440-23-5$ & Sodium & 2.61E1 & ug/g dry & $6.69 \mathrm{E}-1$ & $12 / 05 / 07$ & 8E01004 & PNNL-AGG-ICP-AES \\
\hline HEIS No. & B1PN03 & \multicolumn{3}{|c|}{$\begin{array}{ll}\text { Lab ID: } & \text { 0802020-01 }\end{array}$} & & & \\
\hline $7429-90-5$ & Aluminum & $<8.82 \mathrm{E}-2$ & ug/g dry & $8.82 \mathrm{E}-2$ & $12 / 05 / 07$ & 8E01004 & PNNL-AGG-ICP-AES \\
\hline $7440-70-2$ & Calcium & $1.78 \mathrm{E} 1$ & ug/g dry & $3.98 \mathrm{E}-1$ & $12 / 05 / 07$ & 8E01004 & PNNL-AGG-ICP-AES \\
\hline 7440-09-7 & Potassium & $5.18 \mathrm{E} 0$ & ug/g dry & 2.39E0 & $12 / 05 / 07$ & 8E01004 & PNNL-AGG-ICP-AES \\
\hline 7439-95-4 & Magnesium & 4.27E0 & ug/g dry & $8.58 \mathrm{E}-2$ & $12 / 05 / 07$ & 8E01004 & PNNL-AGG-ICP-AES \\
\hline 7439-96-5 & Manganese & $<1.76 \mathrm{E}-2$ & ug/g dry & $1.76 \mathrm{E}-2$ & $12 / 05 / 07$ & 8E01004 & PNNL-AGG-ICP-AES \\
\hline $7440-23-5$ & Sodium & $1.52 \mathrm{E} 1$ & ug/g dry & $6.88 \mathrm{E}-1$ & $12 / 05 / 07$ & 8E01004 & PNNL-AGG-ICP-AES \\
\hline HEIS No. & B1PN05 & \multicolumn{3}{|c|}{$\begin{array}{ll}\text { Lab ID: } & \text { 0802020-03 }\end{array}$} & & & \\
\hline $7429-90-5$ & Aluminum & $<9.31 \mathrm{E}-2$ & ug/g dry & $9.31 \mathrm{E}-2$ & $12 / 05 / 07$ & 8E01004 & PNNL-AGG-ICP-AES \\
\hline $7440-70-2$ & Calcium & 1.44E1 & ug/g dry & $4.20 \mathrm{E}-1$ & $12 / 05 / 07$ & 8E01004 & PNNL-AGG-ICP-AES \\
\hline 7440-09-7 & Potassium & $5.42 \mathrm{E} 0$ & ug/g dry & 2.53E0 & $12 / 05 / 07$ & 8E01004 & PNNL-AGG-ICP-AES \\
\hline 7439-95-4 & Magnesium & $3.54 \mathrm{E} 0$ & ug/g dry & $9.05 \mathrm{E}-2$ & $12 / 05 / 07$ & 8E01004 & PNNL-AGG-ICP-AES \\
\hline $7439-96-5$ & Manganese & $<1.86 \mathrm{E}-2$ & ug/g dry & $1.86 \mathrm{E}-2$ & $12 / 05 / 07$ & 8E01004 & PNNL-AGG-ICP-AES \\
\hline $7440-23-5$ & Sodium & 1.64E1 & ug/g dry & $7.26 \mathrm{E}-1$ & $12 / 05 / 07$ & 8E01004 & PNNL-AGG-ICP-AES \\
\hline HEIS No. & B1PN07 & \multicolumn{3}{|c|}{$\begin{array}{ll}\text { Lab ID: } & 0802020-05\end{array}$} & & & \\
\hline $7429-90-5$ & Aluminum & $<8.61 \mathrm{E}-2$ & ug/g dry & $8.61 \mathrm{E}-2$ & $12 / 05 / 07$ & 8E01004 & PNNL-AGG-ICP-AES \\
\hline $7440-70-2$ & Calcium & 1.49E1 & ug/g dry & $3.88 \mathrm{E}-1$ & $12 / 05 / 07$ & 8E01004 & PNNL-AGG-ICP-AES \\
\hline 7440-09-7 & Potassium & $5.36 \mathrm{E} 0$ & ug/g dry & 2.34E0 & $12 / 05 / 07$ & 8E01004 & PNNL-AGG-ICP-AES \\
\hline 7439-95-4 & Magnesium & 3.67E0 & ug/g dry & 8.37E-2 & $12 / 05 / 07$ & 8E01004 & PNNL-AGG-ICP-AES \\
\hline $7439-96-5$ & Manganese & $<1.72 \mathrm{E}-2$ & ug/g dry & $1.72 \mathrm{E}-2$ & $12 / 05 / 07$ & 8E01004 & PNNL-AGG-ICP-AES \\
\hline $7440-23-5$ & Sodium & $1.51 \mathrm{E} 1$ & ug/g dry & $6.71 \mathrm{E}-1$ & $12 / 05 / 07$ & 8E01004 & PNNL-AGG-ICP-AES \\
\hline HEIS No. & B1PN09 & \multicolumn{3}{|c|}{ Lab ID: $\quad$ 0802020-09 } & & & \\
\hline $7429-90-5$ & Aluminum & $<8.65 \mathrm{E}-2$ & ug/g dry & 8.65E-2 & $12 / 05 / 07$ & 8E01004 & PNNL-AGG-ICP-AES \\
\hline $7440-70-2$ & Calcium & 1.44E1 & ug/g dry & $3.90 \mathrm{E}-1$ & $12 / 05 / 07$ & 8E01004 & PNNL-AGG-ICP-AES \\
\hline 7440-09-7 & Potassium & 7.23E0 & ug/g dry & $2.35 \mathrm{E} 0$ & $12 / 05 / 07$ & 8E01004 & PNNL-AGG-ICP-AES \\
\hline 7439-95-4 & Magnesium & 3.81E0 & ug/g dry & $8.41 \mathrm{E}-2$ & $12 / 05 / 07$ & 8E01004 & PNNL-AGG-ICP-AES \\
\hline $7439-96-5$ & Manganese & $<1.73 \mathrm{E}-2$ & ug/g dry & $1.73 \mathrm{E}-2$ & $12 / 05 / 07$ & 8E01004 & PNNL-AGG-ICP-AES \\
\hline $7440-23-5$ & Sodium & 2.03E1 & ug/g dry & $6.74 \mathrm{E}-1$ & $12 / 05 / 07$ & 8E01004 & PNNL-AGG-ICP-AES \\
\hline HEIS No. & B1PN11 & \multicolumn{3}{|c|}{$\begin{array}{ll}\text { Lab ID: } & \text { 0802020-11 }\end{array}$} & & & \\
\hline $7429-90-5$ & Aluminum & $<8.83 \mathrm{E}-2$ & ug/g dry & 8.83E-2 & $12 / 05 / 07$ & 8E01004 & PNNL-AGG-ICP-AES \\
\hline $7440-70-2$ & Calcium & $2.44 \mathrm{E} 1$ & ug/g dry & $3.98 \mathrm{E}-1$ & $12 / 05 / 07$ & 8E01004 & PNNL-AGG-ICP-AES \\
\hline 7440-09-7 & Potassium & 6.94E0 & ug/g dry & 2.39E0 & $12 / 05 / 07$ & 8E01004 & PNNL-AGG-ICP-AES \\
\hline 7439-95-4 & Magnesium & 6.47E0 & ug/g dry & $8.58 \mathrm{E}-2$ & $12 / 05 / 07$ & 8E01004 & PNNL-AGG-ICP-AES \\
\hline
\end{tabular}


Total Metals by PNNL-AGG-ICP-AES/Water Extract

\begin{tabular}{|c|c|c|c|c|c|c|c|}
\hline CAS \# & Analyte & Results & Units & EQL & Analyzed & Batch & Method \\
\hline HEIS No. & B1PN11 & \multicolumn{3}{|c|}{ Lab ID: $\quad$ 0802020-11 } & & & \\
\hline $7439-96-5$ & Manganese & $<1.76 \mathrm{E}-2$ & ug/g dry & $1.76 \mathrm{E}-2$ & $12 / 05 / 07$ & 8E01004 & PNNL-AGG-ICP-AES \\
\hline $7440-23-5$ & Sodium & $1.28 \mathrm{E} 1$ & ug/g dry & $6.88 \mathrm{E}-1$ & $12 / 05 / 07$ & 8E01004 & PNNL-AGG-ICP-AES \\
\hline HEIS No. & B1R100 & \multicolumn{3}{|c|}{ Lab ID: $\quad 0802020-17$} & & & \\
\hline $7429-90-5$ & Aluminum & $<8.60 \mathrm{E}-2$ & ug/g dry & $8.60 \mathrm{E}-2$ & $12 / 05 / 07$ & 8E01004 & PNNL-AGG-ICP-AES \\
\hline $7440-70-2$ & Calcium & 7.90E0 & ug/g dry & 3.88E-1 & $12 / 05 / 07$ & 8E01004 & PNNL-AGG-ICP-AES \\
\hline 7440-09-7 & Potassium & 4.59E0 & ug/g dry & 2.33E0 & $12 / 05 / 07$ & 8E01004 & PNNL-AGG-ICP-AES \\
\hline 7439-95-4 & Magnesium & 2.10E0 & ug/g dry & 8.36E-2 & $12 / 05 / 07$ & 8E01004 & PNNL-AGG-ICP-AES \\
\hline 7439-96-5 & Manganese & $<1.72 \mathrm{E}-2$ & ug/g dry & $1.72 \mathrm{E}-2$ & $12 / 05 / 07$ & 8E01004 & PNNL-AGG-ICP-AES \\
\hline $7440-23-5$ & Sodium & 1.02E1 & ug/g dry & $6.71 \mathrm{E}-1$ & $12 / 05 / 07$ & 8E01004 & PNNL-AGG-ICP-AES \\
\hline HEIS No. & B1PN17 & \multicolumn{3}{|c|}{ Lab ID: $\quad$ 0802023-09 } & & & \\
\hline 7429-90-5 & Aluminum & $<8.59 \mathrm{E}-2$ & ug/g dry & 8.59E-2 & $12 / 05 / 07$ & 8E01004 & PNNL-AGG-ICP-AES \\
\hline $7440-70-2$ & Calcium & 6.65E0 & ug/g dry & 3.88E-1 & $12 / 05 / 07$ & 8E01004 & PNNL-AGG-ICP-AES \\
\hline 7440-09-7 & Potassium & 3.07E0 & ug/g dry & 2.33E0 & $12 / 05 / 07$ & 8E01004 & PNNL-AGG-ICP-AES \\
\hline 7439-95-4 & Magnesium & 1.77E0 & ug/g dry & 8.35E-2 & $12 / 05 / 07$ & 8E01004 & PNNL-AGG-ICP-AES \\
\hline 7439-96-5 & Manganese & $<1.72 \mathrm{E}-2$ & ug/g dry & $1.72 \mathrm{E}-2$ & $12 / 05 / 07$ & 8E01004 & PNNL-AGG-ICP-AES \\
\hline $7440-23-5$ & Sodium & 7.40E0 & ug/g dry & $6.70 \mathrm{E}-1$ & $12 / 05 / 07$ & 8E01004 & PNNL-AGG-ICP-AES \\
\hline HEIS No. & B1PN18 & \multicolumn{3}{|c|}{ Lab ID: $\quad$ 0802023-10 } & & & \\
\hline $7429-90-5$ & Aluminum & $<8.61 \mathrm{E}-2$ & ug/g dry & 8.61E-2 & $12 / 05 / 07$ & 8E01004 & PNNL-AGG-ICP-AES \\
\hline $7440-70-2$ & Calcium & 6.47E0 & ug/g dry & 3.89E-1 & $12 / 05 / 07$ & 8E01004 & PNNL-AGG-ICP-AES \\
\hline 7440-09-7 & Potassium & 3.07E0 & ug/g dry & $2.34 \mathrm{E} 0$ & $12 / 05 / 07$ & 8E01004 & PNNL-AGG-ICP-AES \\
\hline 7439-95-4 & Magnesium & $1.70 \mathrm{E} 0$ & ug/g dry & 8.37E-2 & $12 / 05 / 07$ & 8E01004 & PNNL-AGG-ICP-AES \\
\hline 7439-96-5 & Manganese & $<1.72 \mathrm{E}-2$ & ug/g dry & $1.72 \mathrm{E}-2$ & $12 / 05 / 07$ & 8E01004 & PNNL-AGG-ICP-AES \\
\hline $7440-23-5$ & Sodium & 7.31E0 & ug/g dry & $6.72 \mathrm{E}-1$ & $12 / 05 / 07$ & 8E01004 & PNNL-AGG-ICP-AES \\
\hline HEIS No. & B1PN25 & \multicolumn{3}{|c|}{ Lab ID: $\quad$ 0802023-19 } & & & \\
\hline 7429-90-5 & Aluminum & $<8.76 \mathrm{E}-2$ & ug/g dry & 8.76E-2 & $12 / 05 / 07$ & 8E01004 & PNNL-AGG-ICP-AES \\
\hline $7440-70-2$ & Calcium & 7.47E0 & ug/g dry & 3.95E-1 & $12 / 05 / 07$ & 8E01004 & PNNL-AGG-ICP-AES \\
\hline $7440-09-7$ & Potassium & 3.30E0 & ug/g dry & $2.38 \mathrm{E} 0$ & $12 / 05 / 07$ & 8E01004 & PNNL-AGG-ICP-AES \\
\hline 7439-95-4 & Magnesium & 1.95E0 & ug/g dry & $8.51 \mathrm{E}-2$ & $12 / 05 / 07$ & 8E01004 & PNNL-AGG-ICP-AES \\
\hline 7439-96-5 & Manganese & $<1.75 \mathrm{E}-2$ & ug/g dry & $1.75 \mathrm{E}-2$ & $12 / 05 / 07$ & 8E01004 & PNNL-AGG-ICP-AES \\
\hline $7440-23-5$ & Sodium & 6.04E0 & ug/g dry & $6.83 \mathrm{E}-1$ & $12 / 05 / 07$ & 8E01004 & PNNL-AGG-ICP-AES \\
\hline HEIS No. & B1PN26 & \multicolumn{3}{|c|}{ Lab ID: $\quad 0802023-20$} & & & \\
\hline 7429-90-5 & Aluminum & $<8.79 \mathrm{E}-2$ & ug/g dry & 8.79E-2 & $12 / 05 / 07$ & 8E01004 & PNNL-AGG-ICP-AES \\
\hline $7440-70-2$ & Calcium & 6.92E0 & ug/g dry & $3.96 \mathrm{E}-1$ & $12 / 05 / 07$ & 8E01004 & PNNL-AGG-ICP-AES \\
\hline 7440-09-7 & Potassium & 3.27E0 & ug/g dry & 2.39E0 & $12 / 05 / 07$ & 8E01004 & PNNL-AGG-ICP-AES \\
\hline 7439-95-4 & Magnesium & $1.80 \mathrm{E} 0$ & ug/g dry & $8.54 \mathrm{E}-2$ & $12 / 05 / 07$ & 8E01004 & PNNL-AGG-ICP-AES \\
\hline $7439-96-5$ & Manganese & $<1.75 \mathrm{E}-2$ & ug/g dry & $1.75 \mathrm{E}-2$ & $12 / 05 / 07$ & 8E01004 & PNNL-AGG-ICP-AES \\
\hline $7440-23-5$ & Sodium & 6.40E0 & ug/g dry & $6.85 \mathrm{E}-1$ & $12 / 05 / 07$ & 8E01004 & PNNL-AGG-ICP-AES \\
\hline
\end{tabular}




\section{Radionuclides by ICP-MS/Water Extract}

\begin{tabular}{|c|c|c|c|c|c|c|c|}
\hline CAS \# & Analyte & Results & Units & EQL & Analyzed & Batch & Method \\
\hline \multirow{3}{*}{$\begin{array}{l}\text { HEIS No. } \\
\text { 14133-76-7 }\end{array}$} & В1PMY9 & \multicolumn{3}{|c|}{ Lab ID: $\quad$ 0802019-01 } & & & \\
\hline & Technetium-99 & $<9.74 \mathrm{E}-6$ & ug/g dry & $9.74 \mathrm{E}-6$ & $11 / 30 / 07$ & 8D22006 & PNNL-AGG-415 \\
\hline & Uranium 238 & 3.71E-4 & ug/g dry & $2.39 \mathrm{E}-4$ & $11 / 30 / 07$ & 8D22006 & PNNL-AGG-415 \\
\hline \multirow{3}{*}{$\begin{array}{l}\text { HEIS No. } \\
\text { 14133-76-7 }\end{array}$} & B1PN01 & \multicolumn{3}{|c|}{ Lab ID: $\quad$ 0802019-03 } & & & \\
\hline & Technetium-99 & $9.21 \mathrm{E}-6$ & ug/g dry & $9.20 \mathrm{E}-6$ & $11 / 30 / 07$ & 8D22006 & PNNL-AGG-415 \\
\hline & Uranium 238 & $3.29 \mathrm{E}-4$ & ug/g dry & $2.25 \mathrm{E}-4$ & $11 / 30 / 07$ & 8D22006 & PNNL-AGG-415 \\
\hline \multirow{3}{*}{$\begin{array}{l}\text { HEIS No. } \\
14133-76-7\end{array}$} & B1PN03 & \multicolumn{3}{|c|}{ Lab ID: $\quad$ 0802020-01 } & & & \\
\hline & Technetium-99 & $<9.46 \mathrm{E}-6$ & ug/g dry & $9.46 \mathrm{E}-6$ & $11 / 30 / 07$ & 8D22006 & PNNL-AGG-415 \\
\hline & Uranium 238 & $<2.32 \mathrm{E}-4$ & ug/g dry & $2.32 \mathrm{E}-4$ & 11/30/07 & 8D22006 & PNNL-AGG-415 \\
\hline \multirow{3}{*}{$\begin{array}{l}\text { HEIS No. } \\
14133-76-7\end{array}$} & B1PN05 & \multicolumn{3}{|c|}{ Lab ID: $\quad 0802020-03$} & & & \\
\hline & Technetium-99 & $<9.98 \mathrm{E}-6$ & ug/g dry & $9.98 \mathrm{E}-6$ & $11 / 30 / 07$ & 8D22006 & PNNL-AGG-415 \\
\hline & Uranium 238 & $<2.45 \mathrm{E}-4$ & ug/g dry & $2.45 \mathrm{E}-4$ & $11 / 30 / 07$ & 8D22006 & PNNL-AGG-415 \\
\hline \multirow{3}{*}{$\begin{array}{l}\text { HEIS No. } \\
14133-76-7\end{array}$} & B1PN07 & \multicolumn{3}{|c|}{ Lab ID: $\quad$ 0802020-05 } & & & \\
\hline & Technetium-99 & $<9.23 \mathrm{E}-6$ & ug/g dry & $9.23 \mathrm{E}-6$ & $11 / 30 / 07$ & 8D22006 & PNNL-AGG-415 \\
\hline & Uranium 238 & $<2.26 \mathrm{E}-4$ & ug/g dry & $2.26 \mathrm{E}-4$ & $11 / 30 / 07$ & 8D22006 & PNNL-AGG-415 \\
\hline \multirow{3}{*}{$\begin{array}{l}\text { HEIS No. } \\
\text { 14133-76-7 }\end{array}$} & B1PN09 & \multicolumn{3}{|c|}{ Lab ID: $\quad$ 0802020-09 } & & & \\
\hline & Technetium-99 & $9.65 \mathrm{E}-6$ & ug/g dry & $9.27 \mathrm{E}-6$ & $11 / 30 / 07$ & 8D22006 & PNNL-AGG-415 \\
\hline & Uranium 238 & $<2.27 \mathrm{E}-4$ & ug/g dry & $2.27 \mathrm{E}-4$ & $11 / 30 / 07$ & 8D22006 & PNNL-AGG-415 \\
\hline \multirow{3}{*}{$\begin{array}{l}\text { HEIS No. } \\
\text { 14133-76-7 }\end{array}$} & B1PN11 & \multicolumn{3}{|c|}{ Lab ID: $\quad$ 0802020-11 } & & & \\
\hline & Technetium-99 & $1.01 \mathrm{E}-5$ & ug/g dry & $9.46 \mathrm{E}-6$ & $11 / 30 / 07$ & 8D22006 & PNNL-AGG-415 \\
\hline & Uranium 238 & $<2.32 \mathrm{E}-4$ & ug/g dry & $2.32 \mathrm{E}-4$ & $11 / 30 / 07$ & 8D22006 & PNNL-AGG-415 \\
\hline \multirow{3}{*}{$\begin{array}{l}\text { HEIS No. } \\
14133-76-7\end{array}$} & B1R100 & \multicolumn{3}{|c|}{ Lab ID: $\quad$ 0802020-17 } & & & \\
\hline & Technetium-99 & $<9.22 \mathrm{E}-6$ & ug/g dry & $9.22 \mathrm{E}-6$ & $11 / 30 / 07$ & 8D22006 & PNNL-AGG-415 \\
\hline & Uranium 238 & $<2.26 \mathrm{E}-4$ & ug/g dry & $2.26 \mathrm{E}-4$ & $11 / 30 / 07$ & 8D22006 & PNNL-AGG-415 \\
\hline \multirow{3}{*}{$\begin{array}{l}\text { HEIS No. } \\
14133-76-7\end{array}$} & B1PN17 & \multicolumn{3}{|c|}{ Lab ID: $\quad$ 0802023-09 } & & & \\
\hline & Technetium-99 & $1.02 \mathrm{E}-5$ & ug/g dry & $9.21 \mathrm{E}-6$ & $11 / 30 / 07$ & 8D22006 & PNNL-AGG-415 \\
\hline & Uranium 238 & $<2.26 \mathrm{E}-4$ & ug/g dry & $2.26 \mathrm{E}-4$ & $11 / 30 / 07$ & 8D22006 & PNNL-AGG-415 \\
\hline \multirow{3}{*}{$\begin{array}{l}\text { HEIS No. } \\
14133-76-7\end{array}$} & B1PN18 & \multicolumn{3}{|c|}{ Lab ID: $\quad$ 0802023-10 } & & & \\
\hline & Technetium-99 & $<9.24 \mathrm{E}-6$ & ug/g dry & $9.24 \mathrm{E}-6$ & $11 / 30 / 07$ & 8D22006 & PNNL-AGG-415 \\
\hline & Uranium 238 & $<2.26 \mathrm{E}-4$ & ug/g dry & $2.26 \mathrm{E}-4$ & $11 / 30 / 07$ & 8D22006 & PNNL-AGG-415 \\
\hline \multirow{3}{*}{$\begin{array}{l}\text { HEIS No. } \\
14133-76-7\end{array}$} & B1PN25 & \multicolumn{3}{|c|}{ Lab ID: $\quad$ 0802023-19 } & & & \\
\hline & Technetium-99 & $<9.39 \mathrm{E}-6$ & ug/g dry & 9.39E-6 & $11 / 30 / 07$ & 8D22006 & PNNL-AGG-415 \\
\hline & Uranium 238 & $<2.30 \mathrm{E}-4$ & ug/g dry & $2.30 \mathrm{E}-4$ & 11/30/07 & 8D22006 & PNNL-AGG-415 \\
\hline \multirow{3}{*}{$\begin{array}{l}\text { HEIS No. } \\
14133-76-7\end{array}$} & B1PN26 & \multicolumn{3}{|c|}{ Lab ID: $\quad$ 0802023-20 } & & & \\
\hline & Technetium-99 & $1.08 \mathrm{E}-5$ & ug/g dry & 9.43E-6 & $11 / 30 / 07$ & 8D22006 & PNNL-AGG-415 \\
\hline & Uranium 238 & $<2.31 \mathrm{E}-4$ & ug/g dry & $2.31 \mathrm{E}-4$ & $11 / 30 / 07$ & 8D22006 & PNNL-AGG-415 \\
\hline
\end{tabular}


RCRA Metals By PNNL-AGG-415/Water Extract

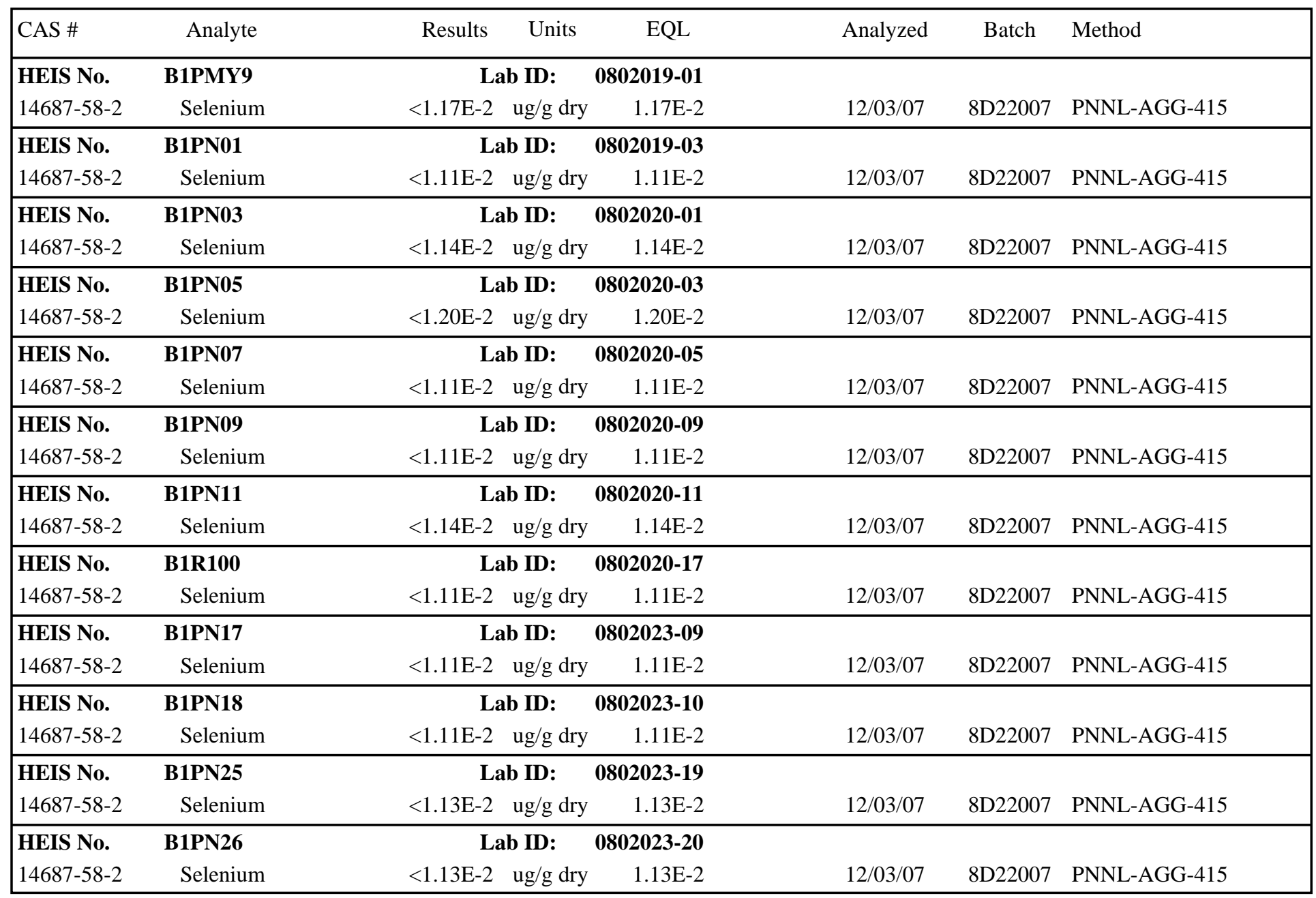


Wet Chemistry - Quality Control

Environmental Science Laboratory

\begin{tabular}{|lrrrrrrrrrrr}
\hline & & Reporting & & Spike & Source & & \%REC & & RPD & & \\
Analyte & Result & Limit & Units & Level & Result & \%REC & Limits & RPD & Limit & Notes \\
\hline
\end{tabular}

Batch 8D07003 - 1:1 Water Extract (pH_EC_Alk)

Blank (8D07003-BLK1)

Alkalinity as $\mathrm{CaCO} 3$

Specific Conductance (EC)

Duplicate (8D07003-DUP1)

Specific Conductance (EC)

Alkalinity as $\mathrm{CaCO} 3$

P1)

Batch 8D30002 - 1:1 Water Extract (pH_EC_Alk)

Duplicate (8D30002-DUP1)

Duplicate (8D30002-DUP2)

$\mathrm{pH}$
Prepared: 11/27/07 Analyzed: 11/29/07

$\begin{array}{rrr}<2.35 \mathrm{E} 1 & 2.35 \mathrm{E} 1 & \mathrm{ug} / \mathrm{g} \text { wet } \\ <1.00 \mathrm{E}-2 & 1.00 \mathrm{E}-2 & \mathrm{mS} / \mathrm{cm}\end{array}$

Source: 0802019-03

Prepared: 11/27/07 Analyzed: 11/28/07

$\begin{array}{lcc}3.22 \mathrm{E}-1 & 0.623 & 35 \\ 4.18 \mathrm{E} 1 & 16.3 & 35\end{array}$

$4.18 \mathrm{E} 1$

35

$\begin{array}{rrr}3.20 \mathrm{E}-1 & 1.00 \mathrm{E}-2 & \mathrm{mS} / \mathrm{cm} \\ 3.55 \mathrm{E} 1 & 2.39 \mathrm{E} 1 & \mathrm{ug} / \mathrm{g} \text { dry }\end{array}$

$\begin{array}{ccc}\text { Source: 0802019-03 } & \text { Prepared \& Analyzed: 11/28/07 } \\ 0 & \text { N/A } \quad \text { pH Units } & 7.23 E 0\end{array}$

\begin{tabular}{ccc} 
Source: $\mathbf{0 8 0 2 0 2 0 - 0 1}$ & Prepared \& Analyzed: 11/28/07 \\
\hline N/A & pH Units & $7.45 E 0$
\end{tabular}

$4.46 \quad 35$

$0.00 \quad 35$


Anions by Ion Chromatography - Quality Control

Environmental Science Laboratory

\begin{tabular}{|c|c|c|c|c|c|c|c|c|c|c|}
\hline Analyte & Result & $\begin{array}{r}\text { porting } \\
\text { Limit }\end{array}$ & Units & $\begin{array}{l}\text { Spike } \\
\text { Level }\end{array}$ & $\begin{array}{l}\text { Source } \\
\text { Result }\end{array}$ & \%REC & $\begin{array}{l}\text { \%REC } \\
\text { Limits }\end{array}$ & RPD & $\begin{array}{l}\text { RPD } \\
\text { Limit }\end{array}$ & Notes \\
\hline
\end{tabular}

Batch 8D25002 - 1:1 Water Extract (IC)

\begin{tabular}{|c|c|c|c|c|c|c|c|c|c|}
\hline \multirow{2}{*}{$\begin{array}{l}\text { Blank (8D25002-BLK1) } \\
\text { Fluoride }\end{array}$} & \multicolumn{9}{|c|}{ Prepared: 11/28/07 Analyzed: 11/29/07 } \\
\hline & $<2.00 \mathrm{E} 0$ & $2.00 \mathrm{E} 0$ & ug/g wet & & & & & & \\
\hline Nitrite & $<1.00 \mathrm{E} 1$ & $1.00 \mathrm{E} 1$ & " & & & & & & \\
\hline Nitrate & $<1.00 \mathrm{E} 1$ & $1.00 \mathrm{E} 1$ & " & & & & & & \\
\hline Sulfate & $<1.50 \mathrm{E} 1$ & $1.50 \mathrm{E} 1$ & " & & & & & & \\
\hline Phosphate & $<1.50 \mathrm{E} 1$ & $1.50 \mathrm{E} 1$ & $"$ & & & & & & \\
\hline LCS (8D25002-BS1) & \multicolumn{9}{|c|}{ Prepared: 11/28/07 Analyzed: 11/29/07 } \\
\hline Fluoride & $1.98 \mathrm{E} 0$ & $2.00 \mathrm{E}-1$ & ug/g wet & $2.00 \mathrm{E} 0$ & & 99.2 & $80-120$ & & \\
\hline Nitrite & $1.02 \mathrm{E} 1$ & $1.00 \mathrm{E} 0$ & $"$ & $1.00 \mathrm{E} 1$ & & 102 & $80-120$ & & \\
\hline Nitrate & $9.75 \mathrm{E} 0$ & $1.00 \mathrm{E} 0$ & $"$ & $1.00 \mathrm{E} 1$ & & 97.2 & $80-120$ & & \\
\hline Sulfate & $1.39 \mathrm{E} 1$ & $1.50 \mathrm{E} 0$ & $"$ & $1.51 \mathrm{E} 1$ & & 92.5 & $80-120$ & & \\
\hline Phosphate & $1.41 \mathrm{E} 1$ & $1.50 \mathrm{E} 0$ & $"$ & $1.51 \mathrm{E} 1$ & & 93.5 & $80-120$ & & \\
\hline Duplicate (8D25002-DUP1) & \multicolumn{3}{|c|}{ Source: 0802019-03 } & \multicolumn{6}{|c|}{ Prepared: 11/28/07 Analyzed: 11/29/07 } \\
\hline Fluoride & $<2.03 \mathrm{E} 0$ & $2.03 \mathrm{E} 0$ & ug/g dry & & ND & & & & 35 \\
\hline Nitrite & $<1.02 \mathrm{E} 1$ & $1.02 \mathrm{E} 1$ & $"$ & & ND & & & & 35 \\
\hline Nitrate & $<1.02 \mathrm{E} 1$ & $1.02 \mathrm{E} 1$ & $"$ & & $9.45 \mathrm{E} 0$ & & & & 35 \\
\hline Sulfate & 9.03E1 & $1.52 \mathrm{E} 1$ & $"$ & & $8.41 \mathrm{E} 1$ & & & 7.11 & 35 \\
\hline Phosphate & $<1.52 \mathrm{E} 1$ & $1.52 \mathrm{E} 1$ & $"$ & & ND & & & & 35 \\
\hline Post Spike (8D25002-PS1) & \multicolumn{3}{|c|}{ Source: 0802023-19 } & \multicolumn{4}{|c|}{ Prepared \& Analyzed: 11/28/07 } & & \\
\hline Fluoride & 4.13E-1 & N/A & $\mathrm{ug} / \mathrm{mL}$ & $4.00 \mathrm{E}-1$ & ND & 105 & $75-125$ & & \\
\hline Nitrite & $2.04 \mathrm{E} 0$ & N/A & $"$ & $2.00 \mathrm{E} 0$ & ND & 102 & $75-125$ & & \\
\hline Nitrate & 2.13E1 & N/A & $"$ & $2.00 \mathrm{E} 1$ & $8.06 \mathrm{E}-1$ & 102 & $75-125$ & & \\
\hline Sulfate & 4.01E0 & N/A & " & $3.00 \mathrm{E} 0$ & $1.43 \mathrm{E} 0$ & 86.1 & $75-125$ & & \\
\hline Phosphate & $3.04 \mathrm{E} 0$ & N/A & $"$ & 3.00E0 & 3.79E-1 & 88.6 & $75-125$ & & \\
\hline
\end{tabular}


Total Metals by PNNL-AGG-ICP-AES/Water Extract - Quality Control

Environmental Science Laboratory

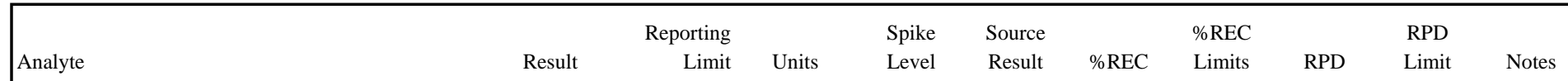

Batch 8E01004 - 1:1 Water Extract (ICP/ICPMS)

Blank (8E01004-BLK1)

Aluminum

Calcium

Chromium

Potassium

Magnesium

Manganese

Sodium

LCS (8E01004-BS1)

Aluminum

Calcium

Chromium

Potassium

Magnesium

Manganese

Sodium

Duplicate (8E01004-DUP1)

1004-BS1)

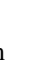

Aluminum

Calcium

Chromium

Potassium

Magnesium

Manganese

Sodium

Post Spike (8E01004-PS1)

Aluminum

Calcium

Potassium

Magnesium

Manganese

Sodium

(8E01004-DUP1)

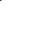

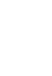




\section{Radionuclides by ICP-MS/Water Extract - Quality Control \\ Environmental Science Laboratory}

\begin{tabular}{|lrrrrrrrrrr}
\hline & & Reporting & & Spike & Source & & \%REC & & RPD & \\
Analyte & Result & Limit & Units & Level & Result & \%REC & Limits & RPD & Limit & Notes \\
\hline
\end{tabular}

Batch 8D22006 - 1:1 Water Extract (ICP/ICPMS)

Blank (8D22006-BLK1)

Technetium-99

Uranium 238

Duplicate (8D22006-DUP1)

Technetium-99

Uranium 238

Post Spike (8D22006-PS1)

Technetium-99

Uranium 238

$\begin{array}{ccc}<9.20 \mathrm{E}-6 & 9.20 \mathrm{E}-6 & \text { ug/g wet } \\ <2.25 \mathrm{E}-4 & 2.25 \mathrm{E}-4 & "\end{array}$

Prepared \& Analyzed: 11/30/07

Source: 0802019-03

$<9.35 \mathrm{E}-6 \quad 9.35 \mathrm{E}-6 \quad$ ug/g dry

3.46E-4

$2.29 \mathrm{E}-4$

Source: 0802019-03

5.05E-1

$6.70 \mathrm{E}-1$
Prepared \& Analyzed: 11/30/07

$\begin{array}{lll}9.21 \mathrm{E}-6 & & 35 \\ 3.29 \mathrm{E}-4 & 5.06 & 35\end{array}$

Prepared \& Analyzed: 11/30/07

$\begin{array}{lccccc}\text { N/A } & \text { ug/L } & 5.00 \mathrm{E}-1 & 4.61 \mathrm{E}-3 & 100 & 75-125 \\ \text { N/A } & " & 5.00 \mathrm{E}-1 & 1.64 \mathrm{E}-1 & 101 & 75-125\end{array}$


RCRA Metals By PNNL-AGG-415/Water Extract - Quality Control

Environmental Science Laboratory

\begin{tabular}{|lrrrrrrrrrrr}
\hline & & Reporting & & Spike & Source & & \%REC & & RPD & & \\
Analyte & Result & Limit & Units & Level & Result & \%REC & Limits & RPD & Limit & Notes \\
\hline
\end{tabular}

Batch 8D22007 - 1:1 Water Extract (ICP/ICPMS)

Blank (8D22007-BLK1)

Selenium

$<1.10 \mathrm{E}-2 \quad 1.10 \mathrm{E}-2 \quad \mathrm{ug} / \mathrm{g}$ wet

Prepared \& Analyzed: 12/03/07

LCS (8D22007-BS1)

Prepared \& Analyzed: 12/03/07

Selenium

4.20E0

1.10E0 ug/g wet $\quad 5.00 \mathrm{E} 0$

80-120

Duplicate (8D22007-DUP1)

Selenium

Source: 0802019-03

Prepared \& Analyzed: 12/03/07

$<1.12 \mathrm{E}-2 \quad 1.12 \mathrm{E}-2 \quad$ ug/g dry

ND 


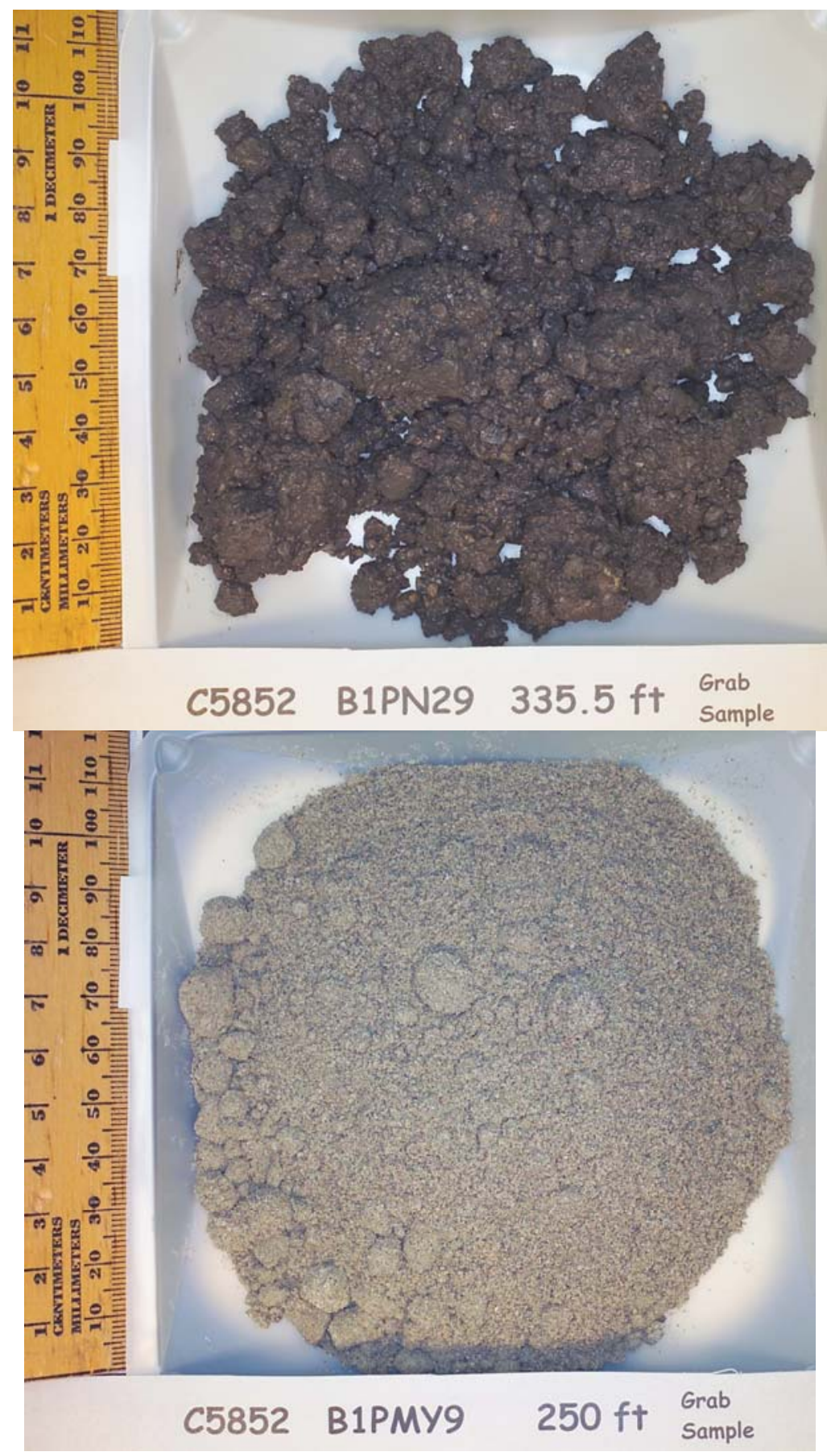



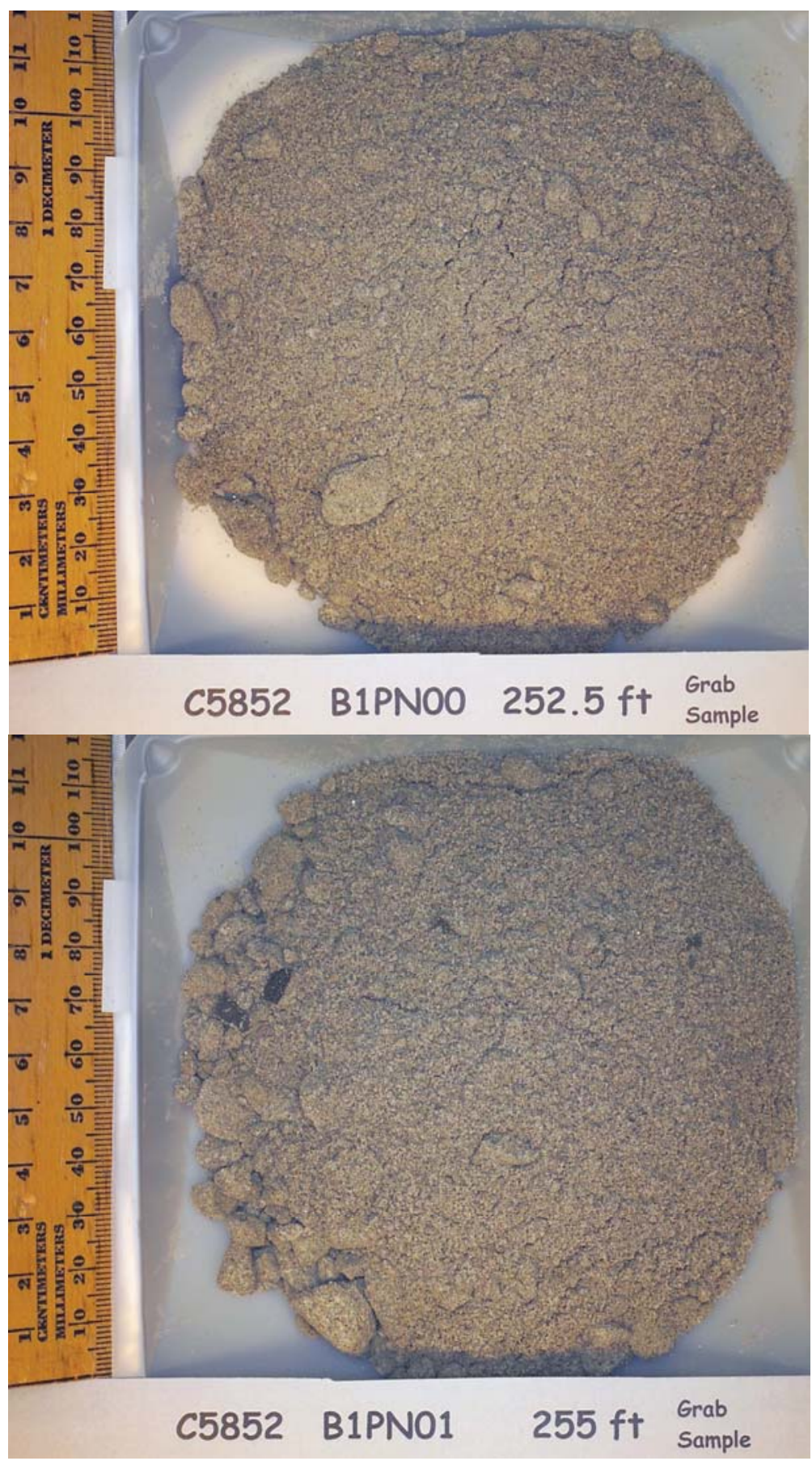


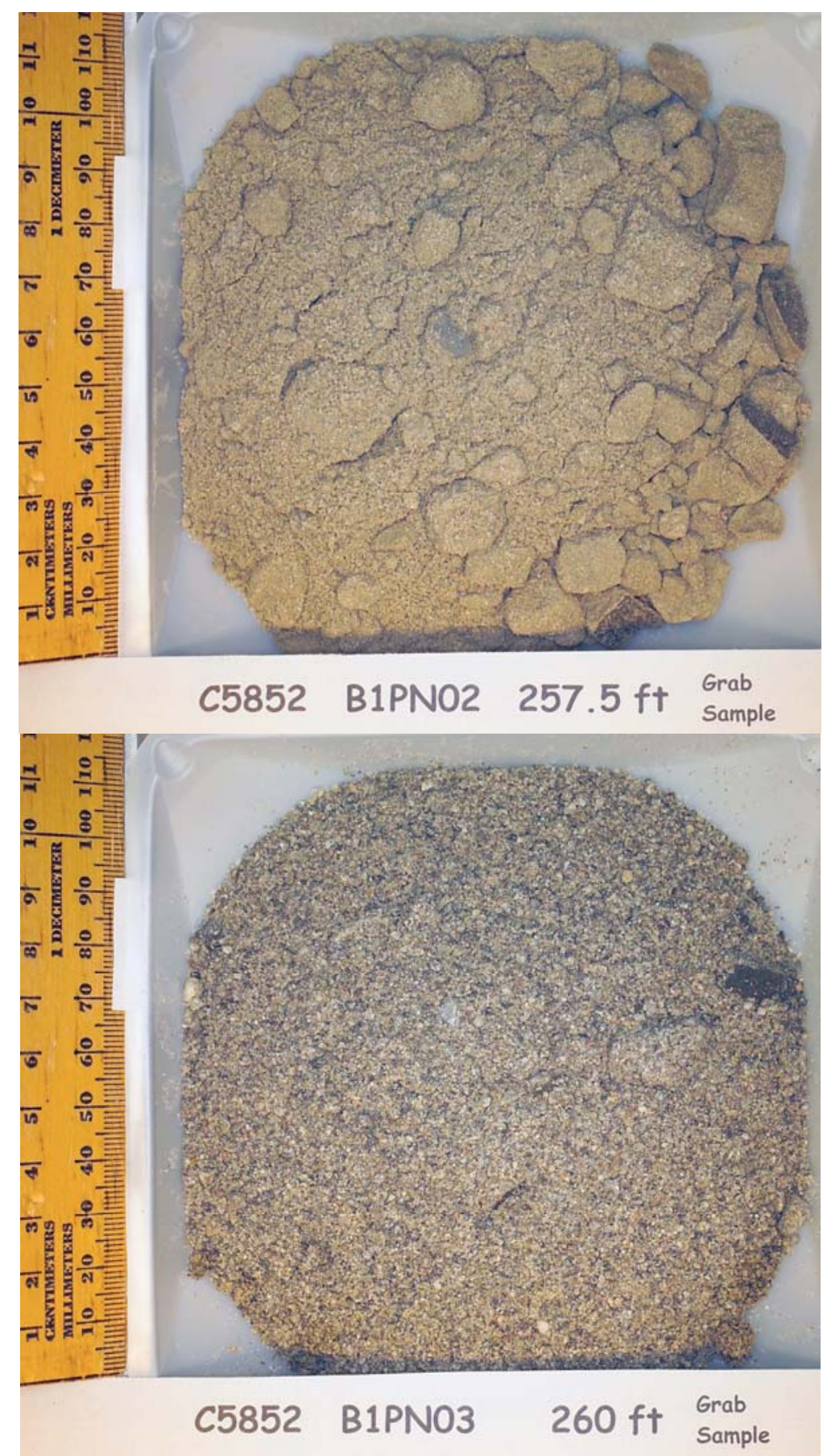




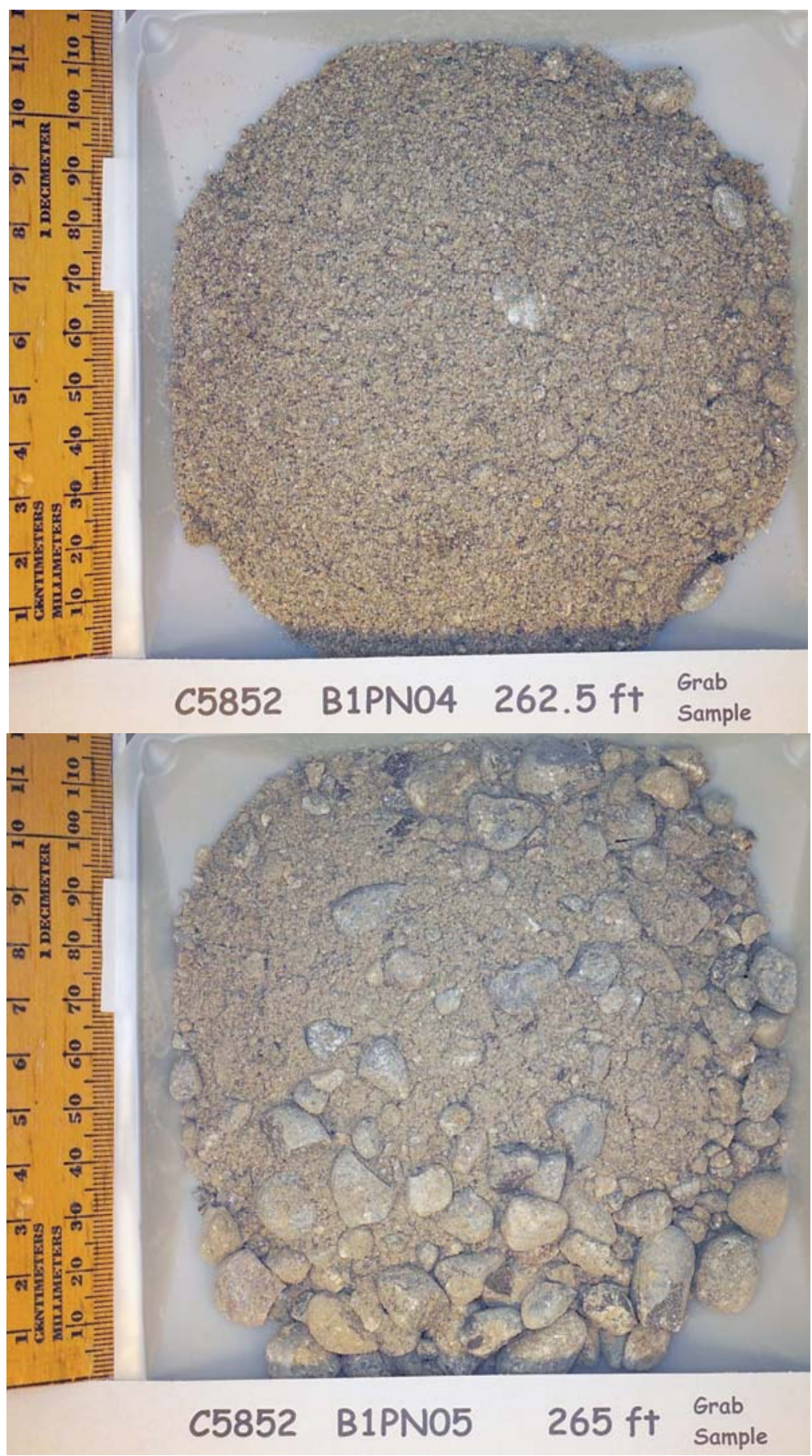




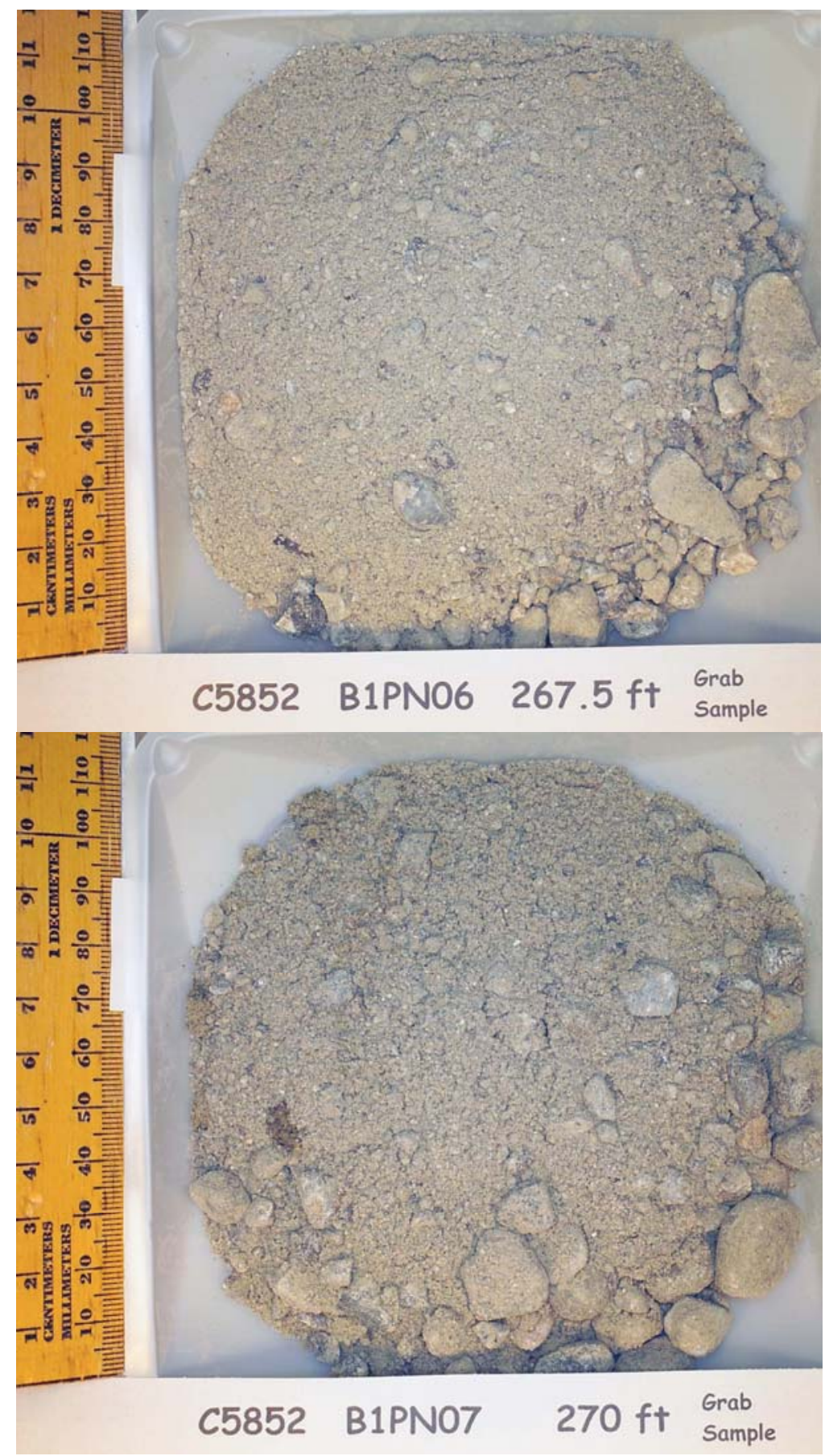




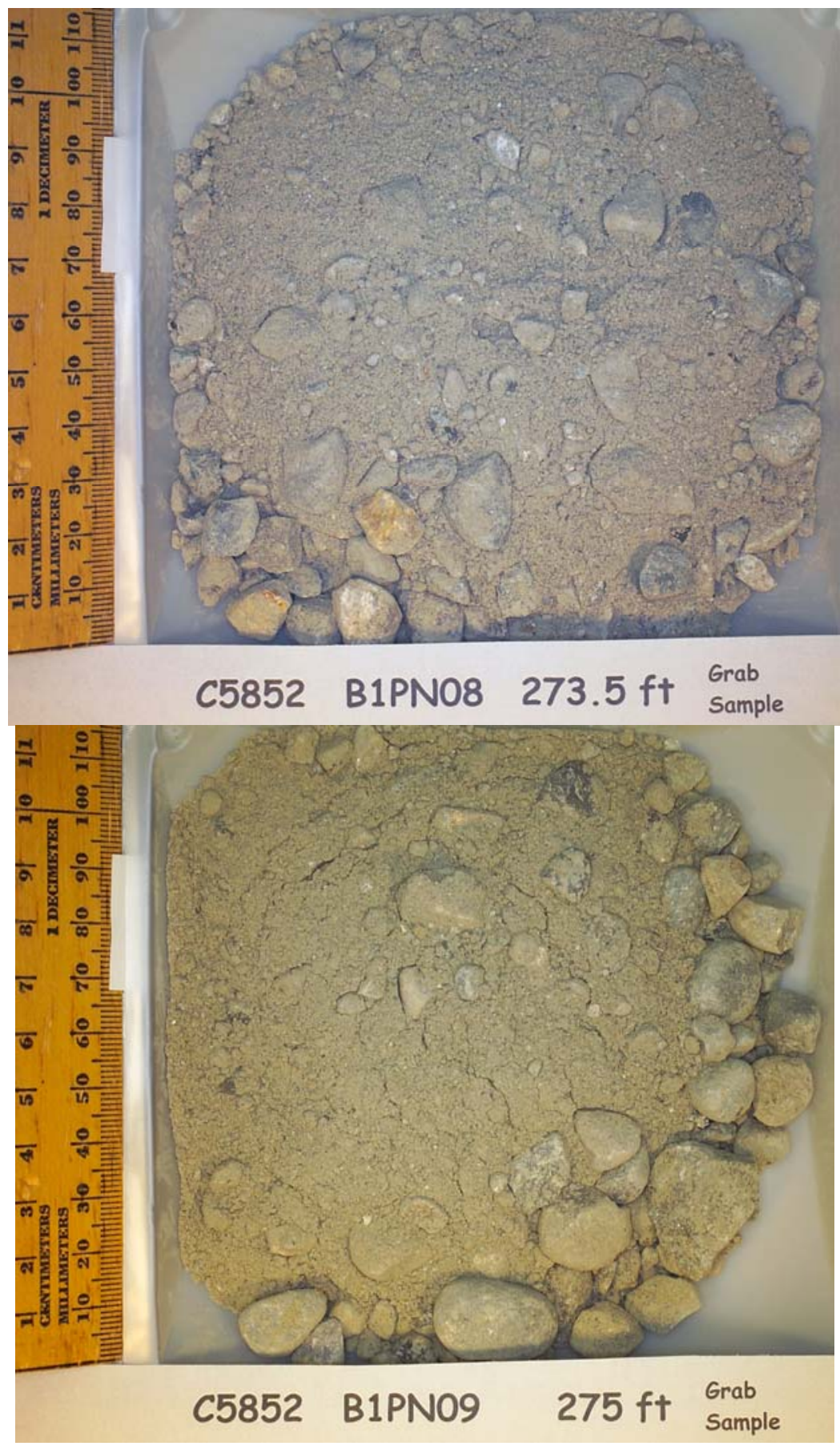




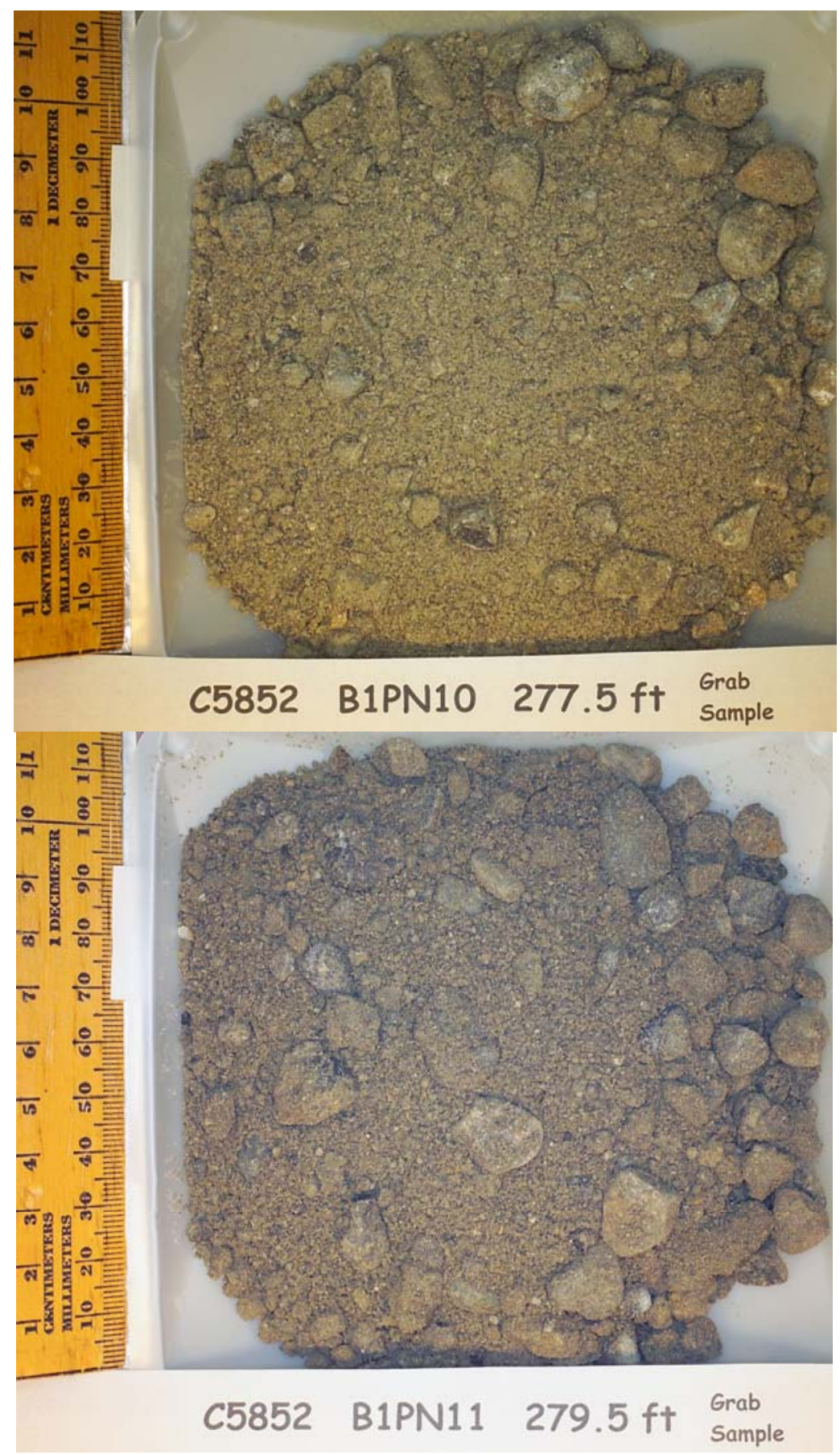




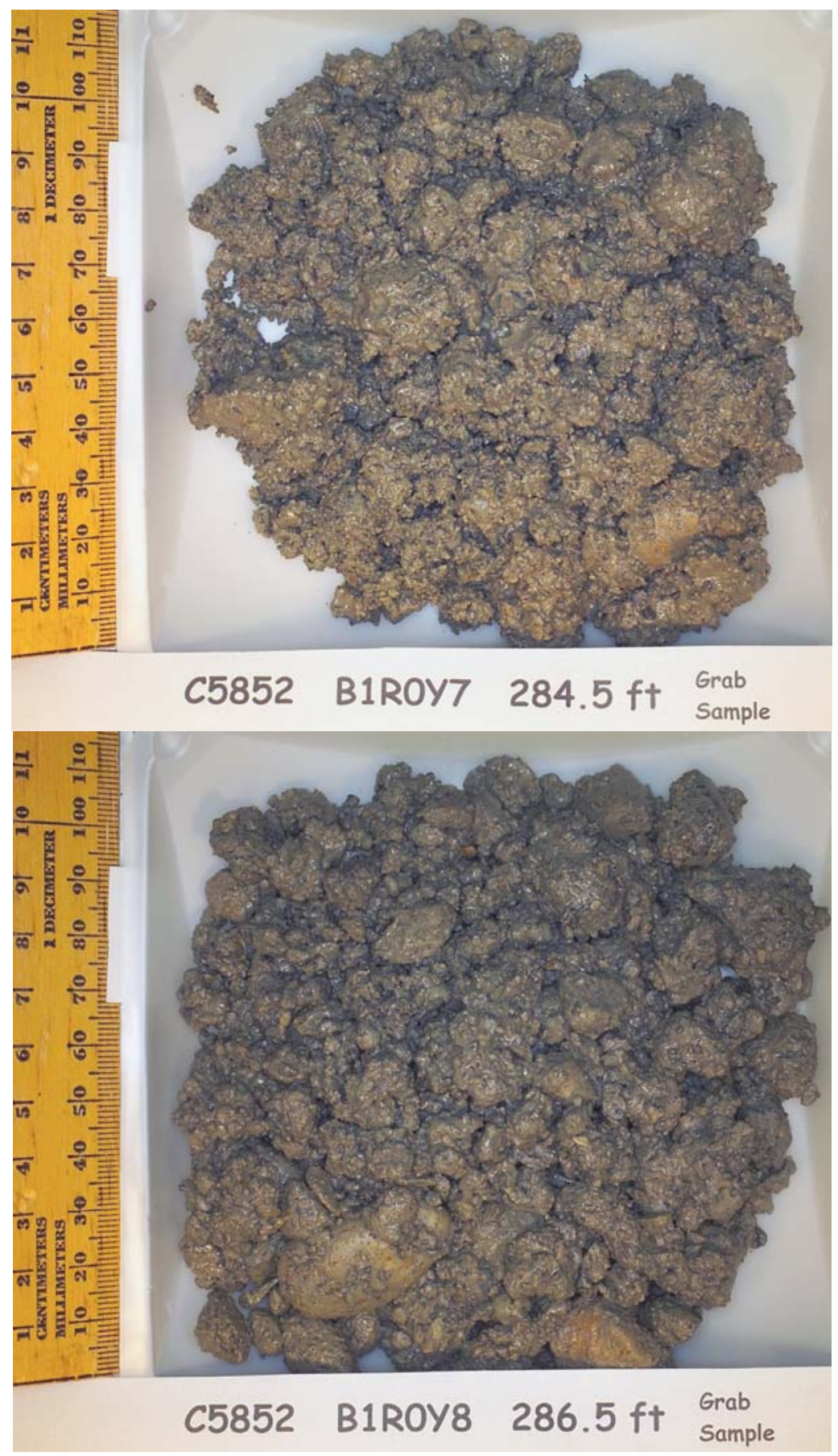




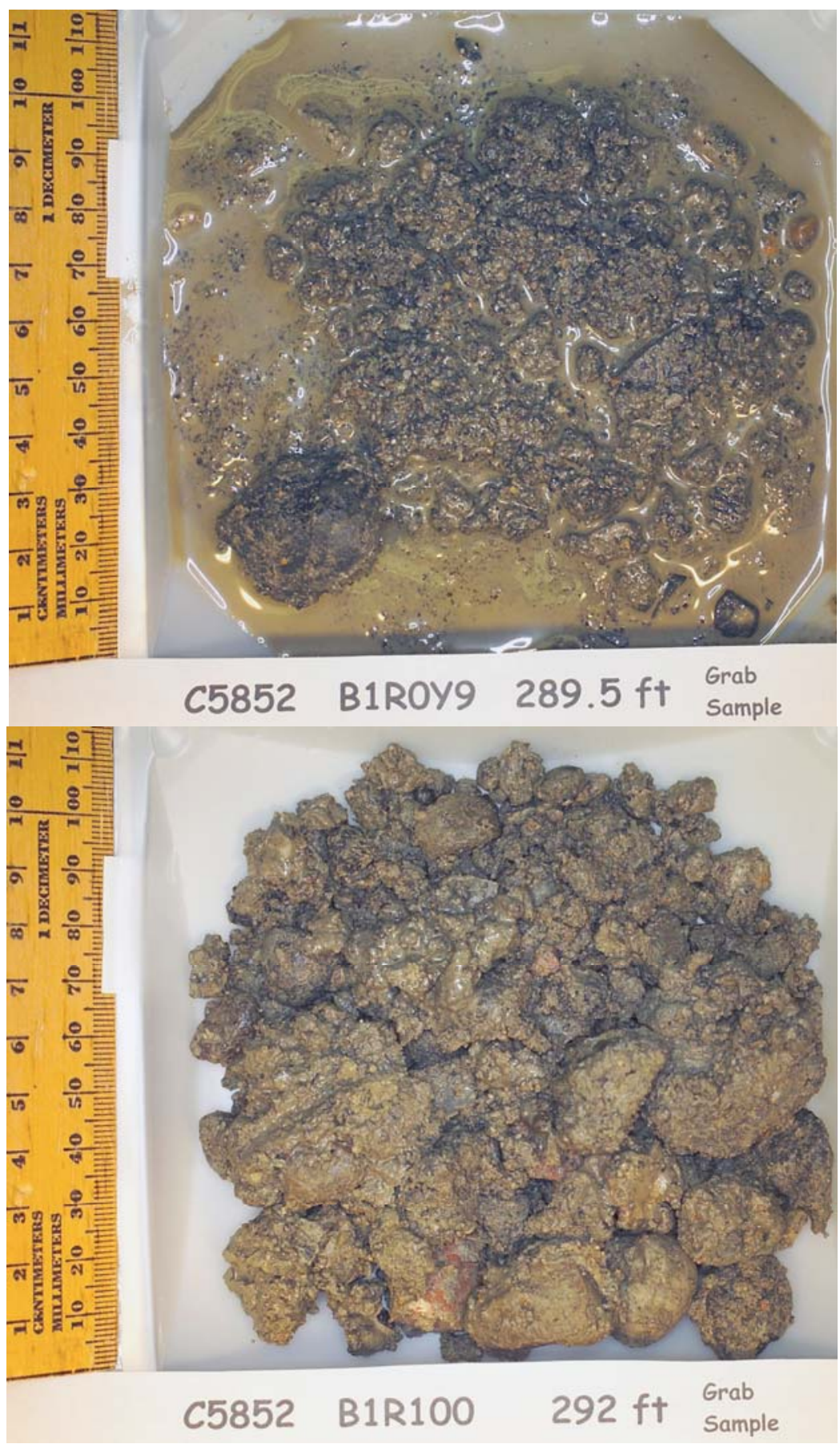




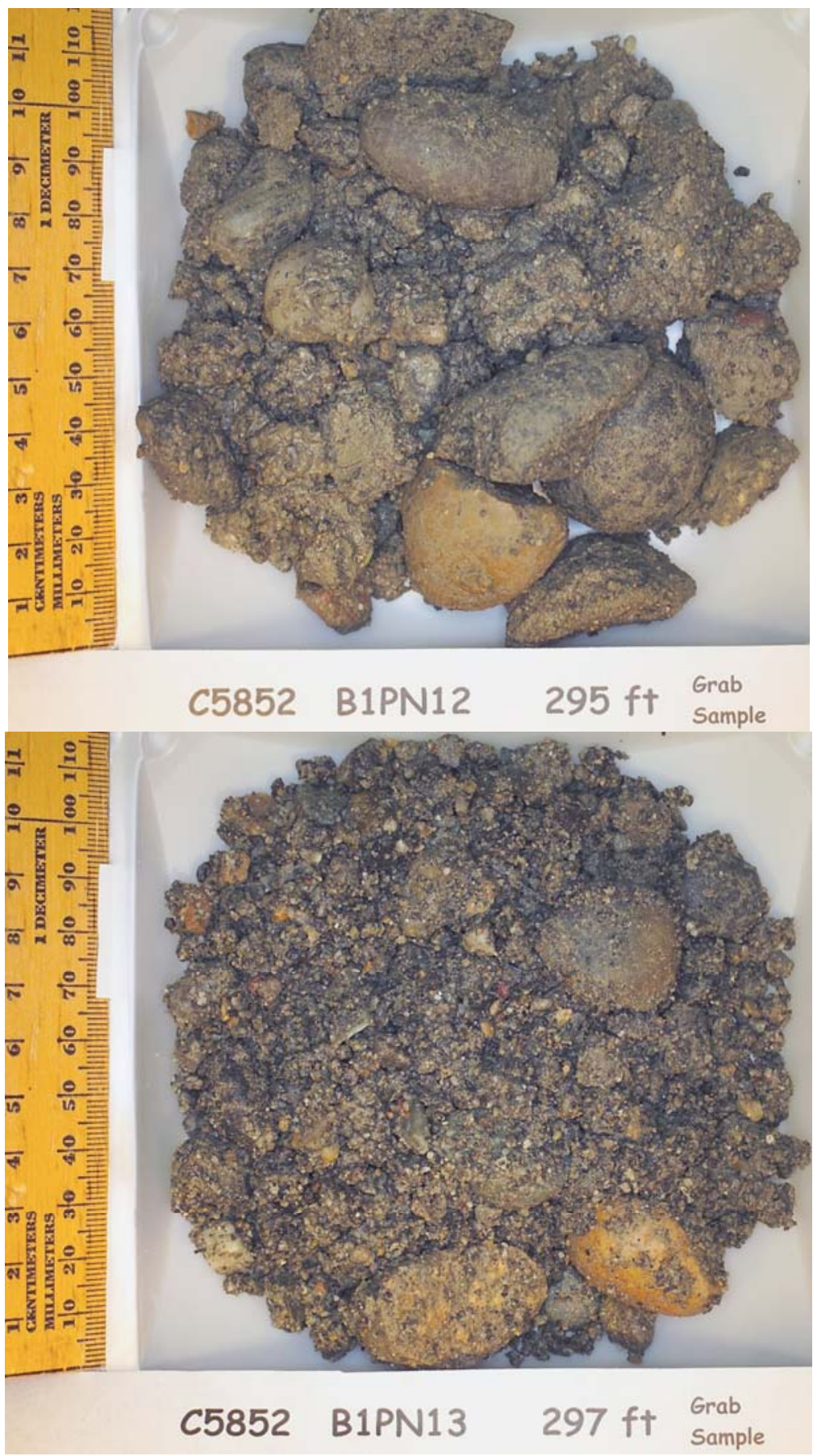




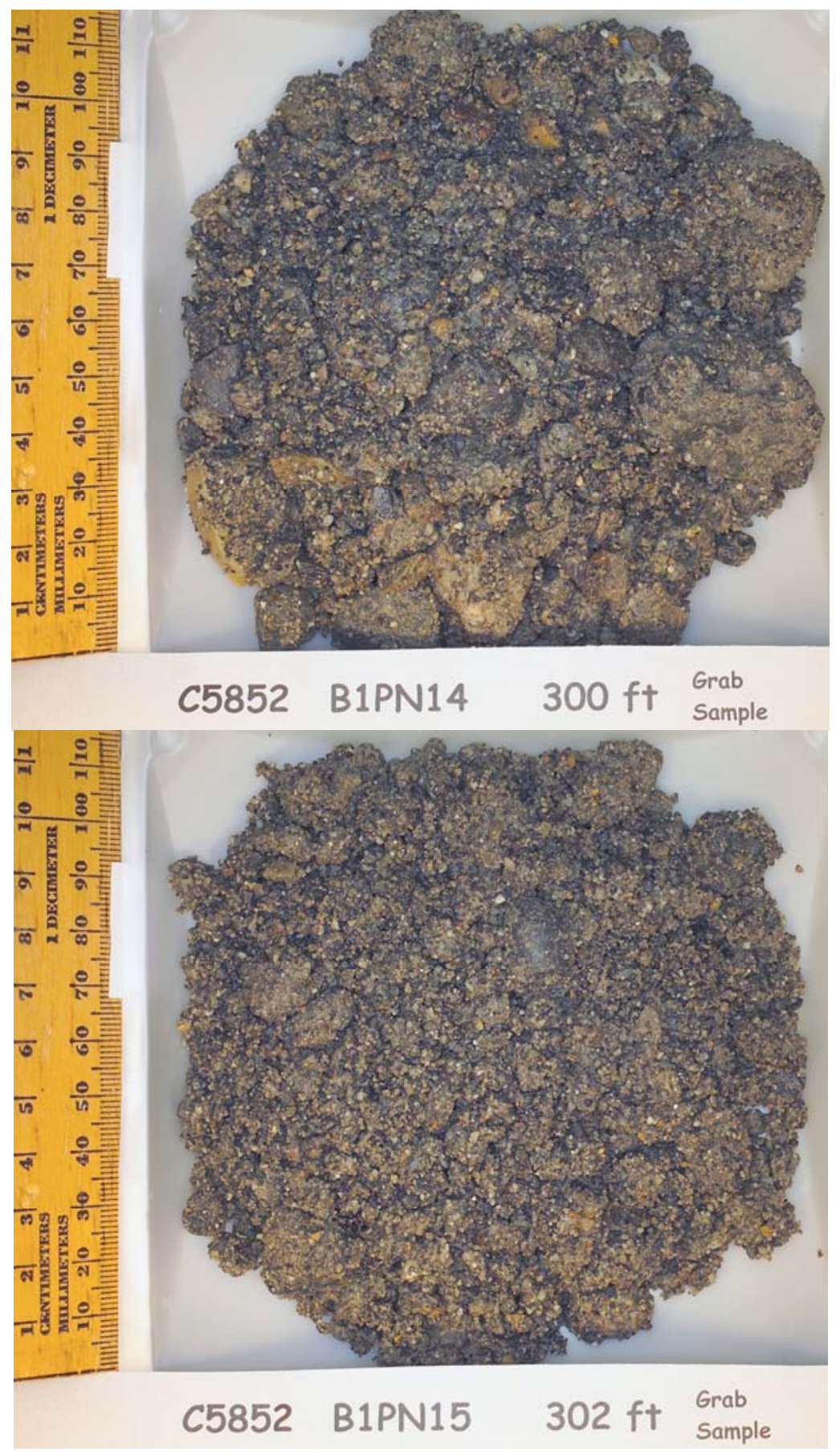




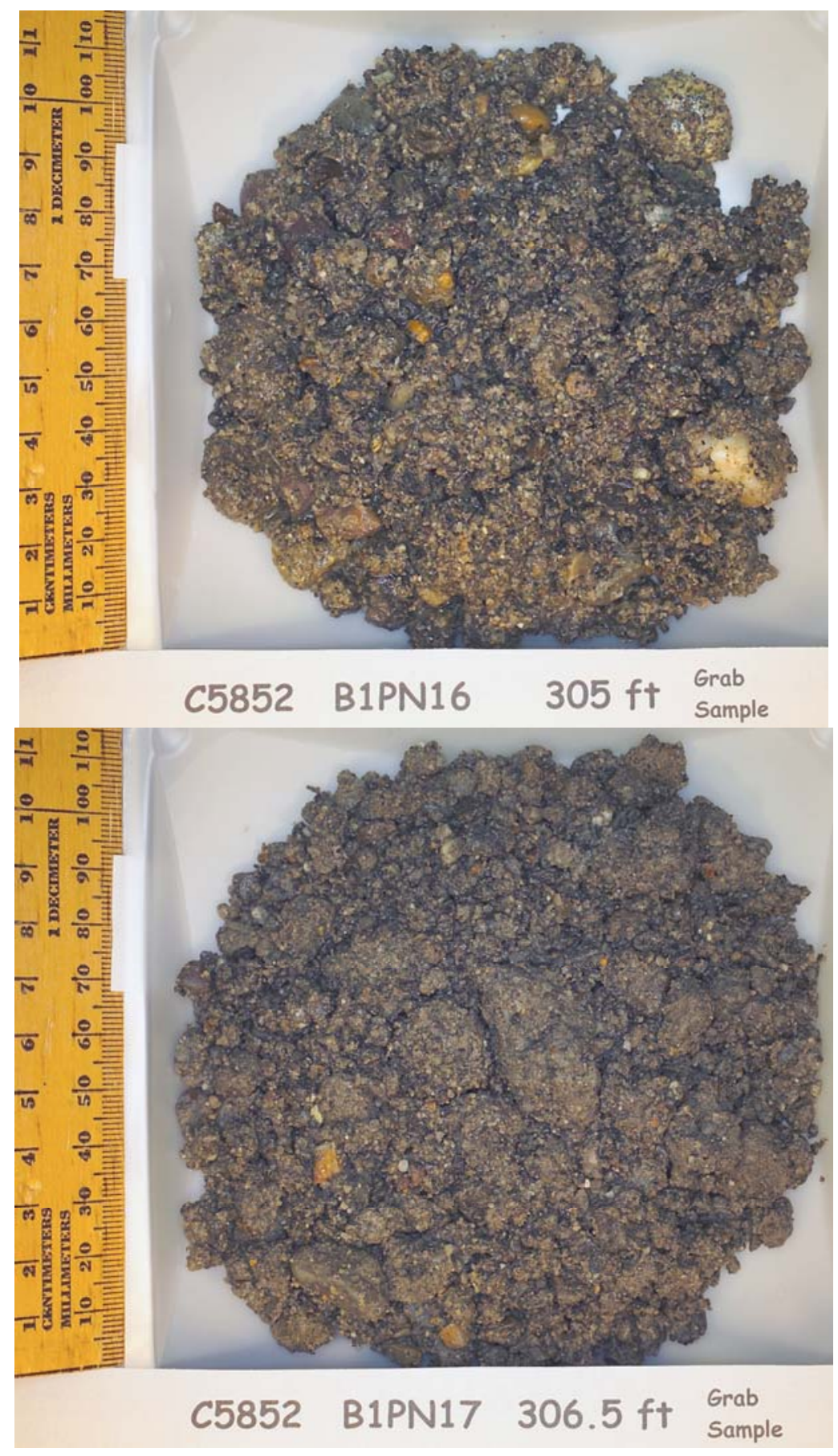




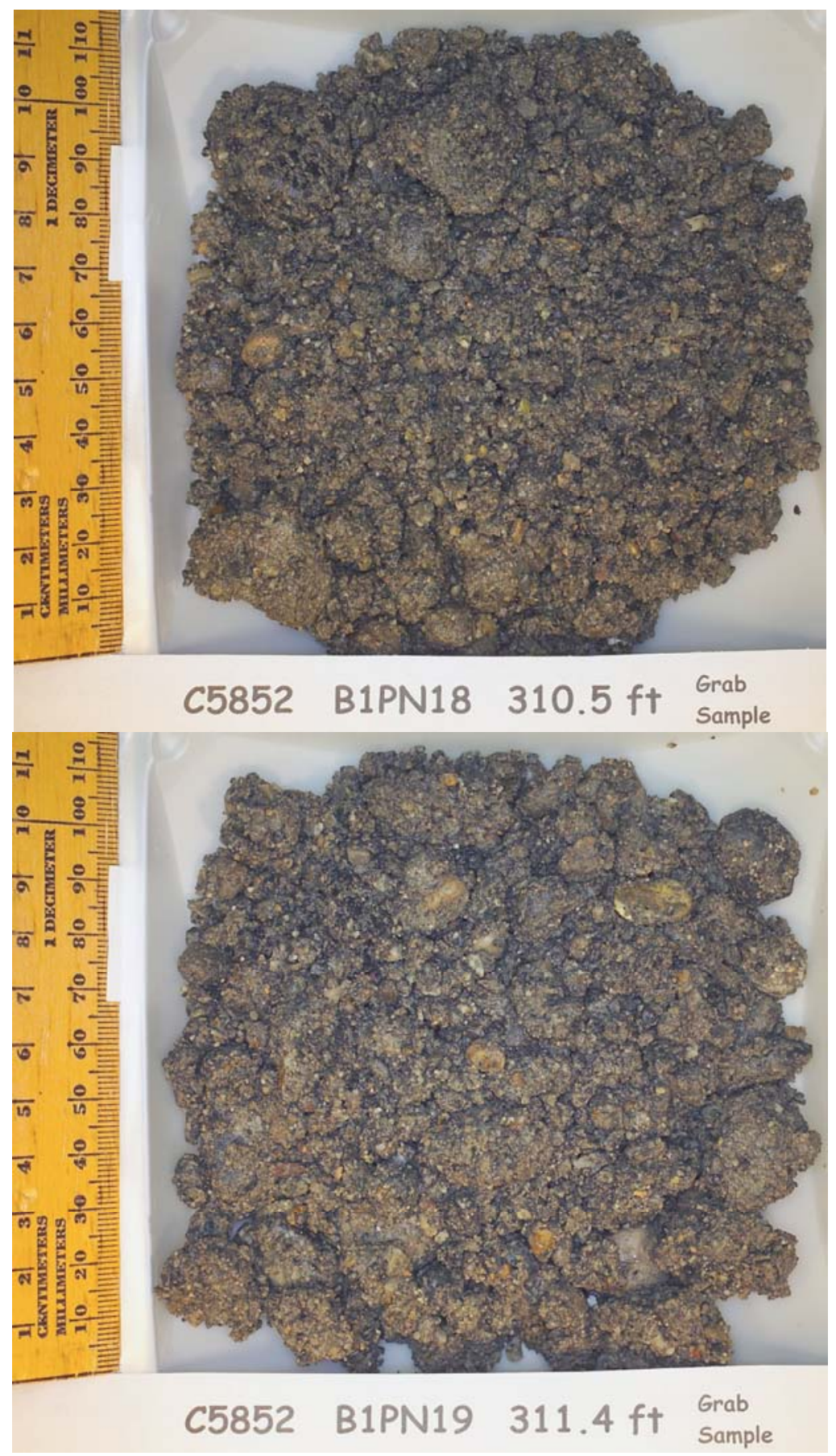




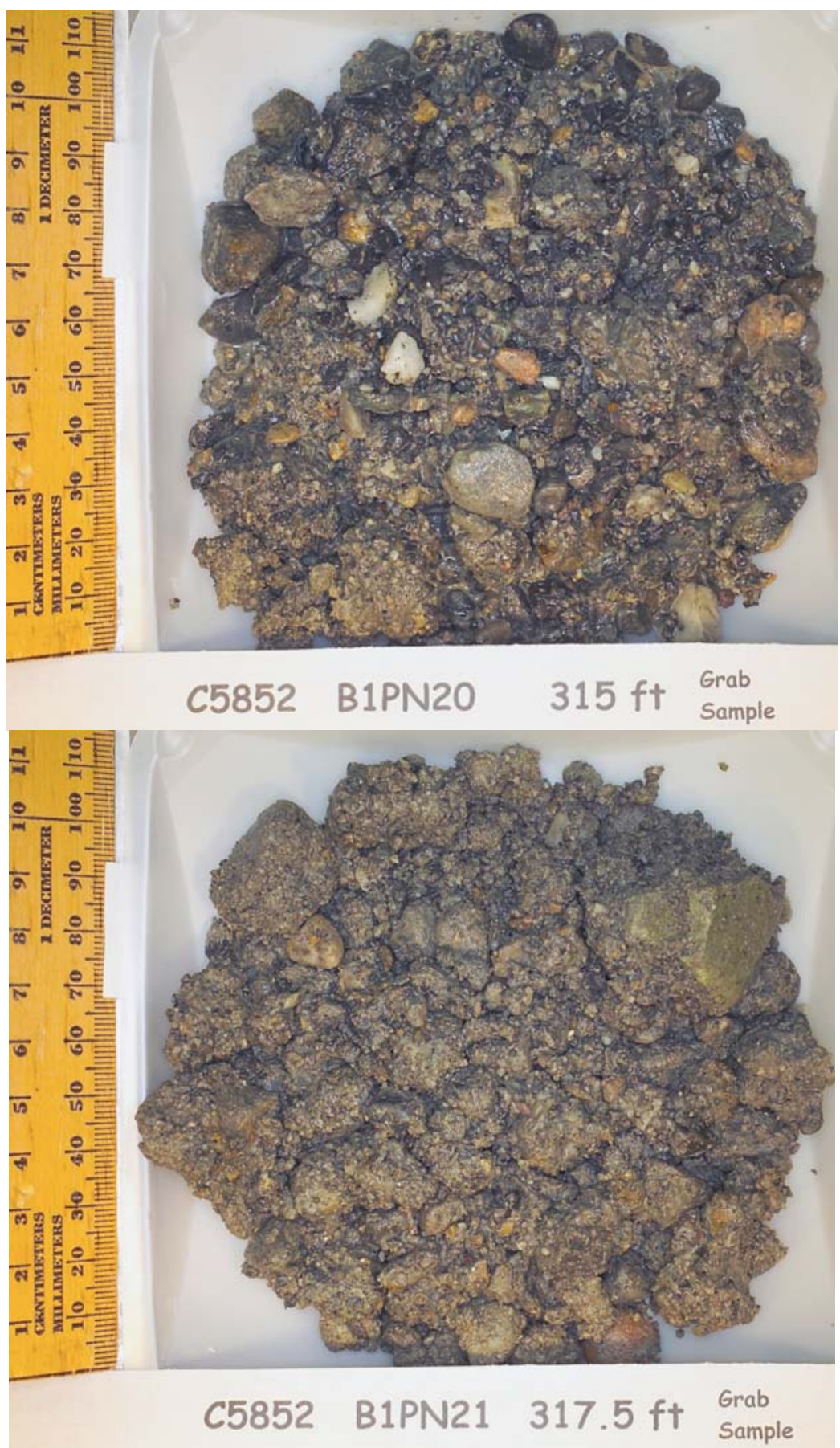




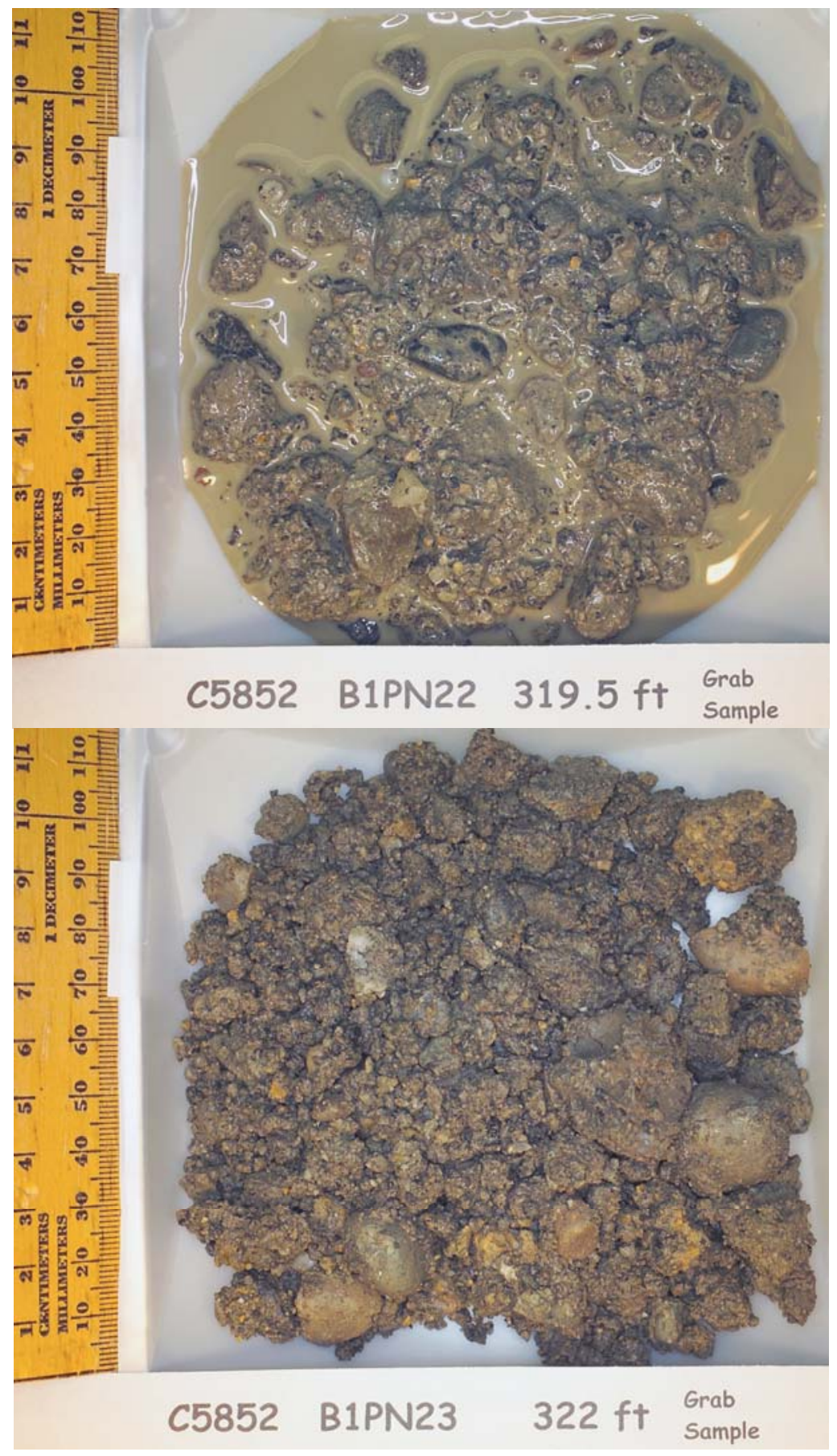




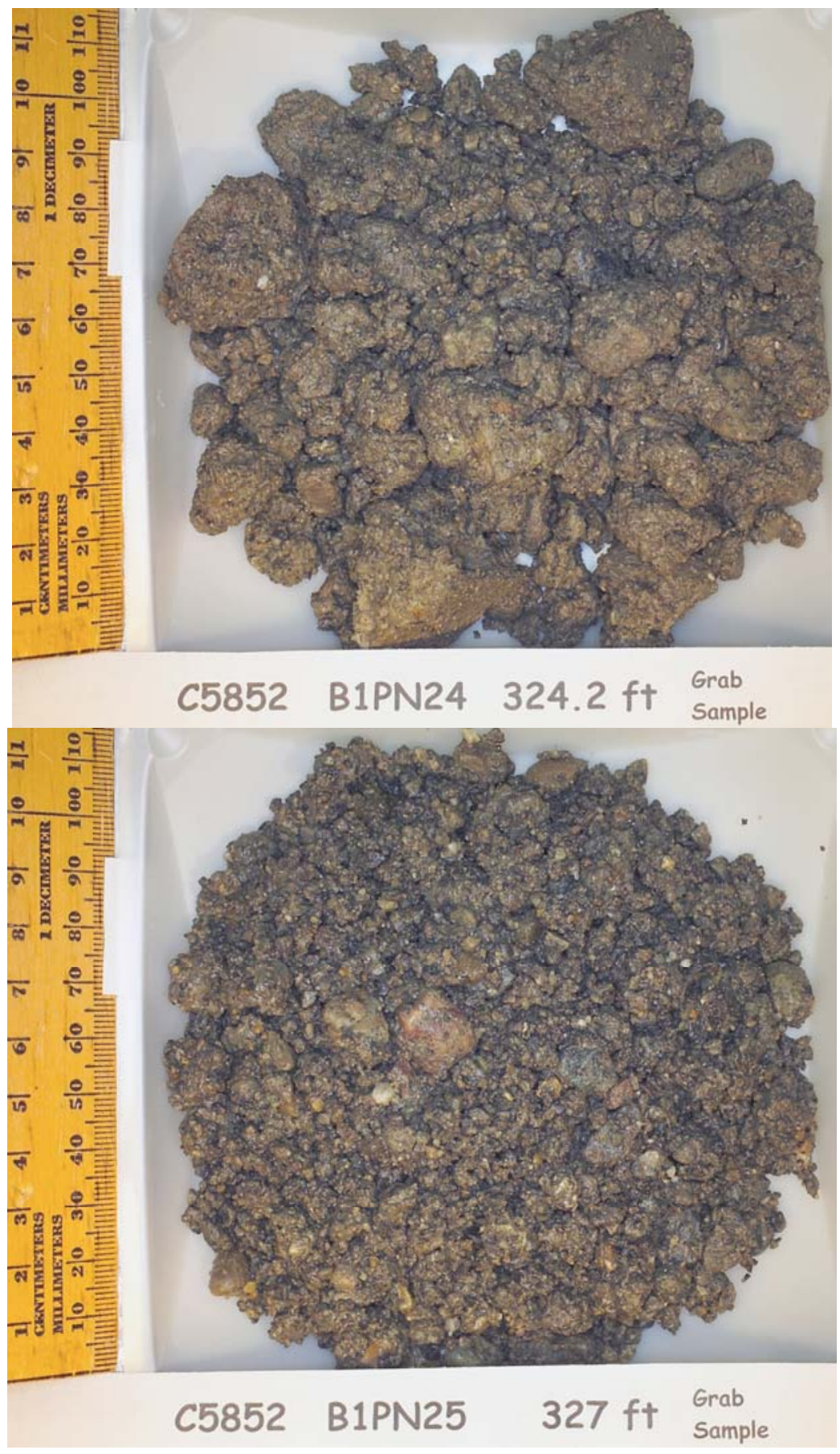




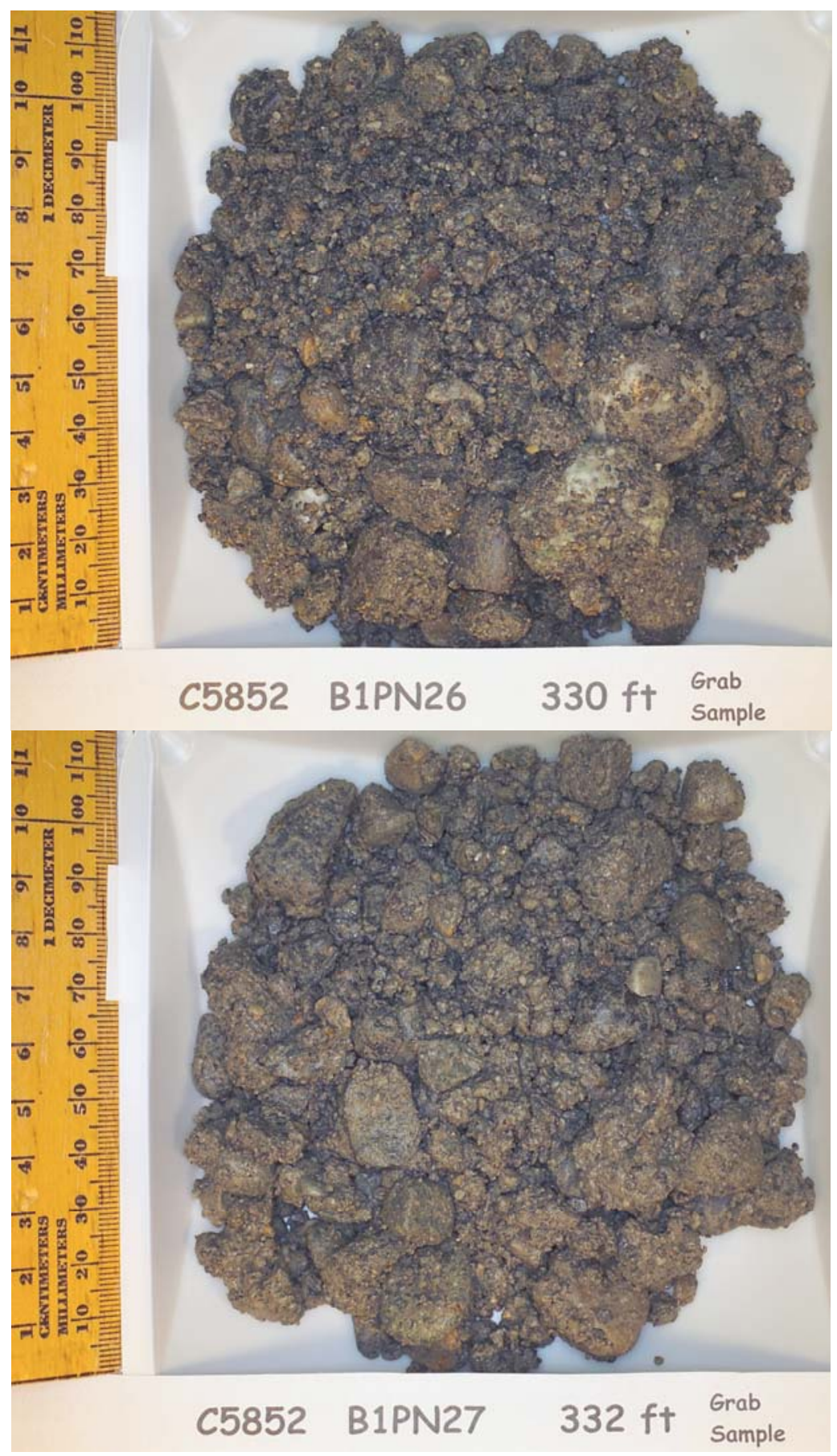



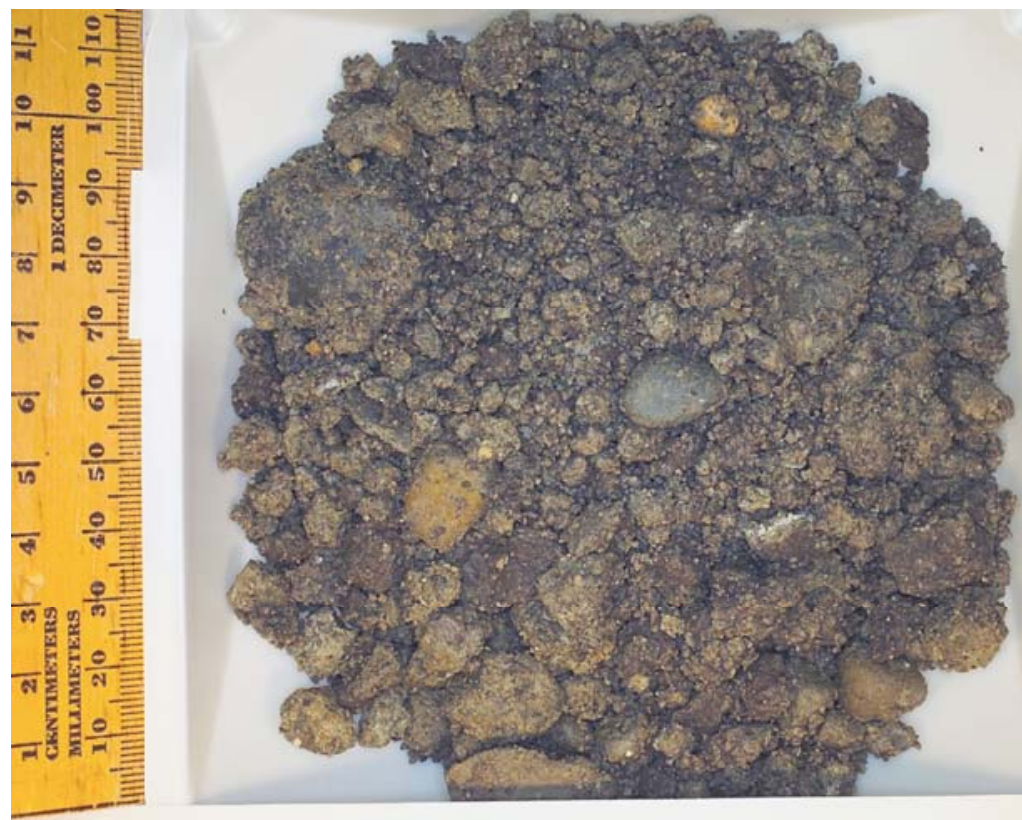

C5852 B1PN28 $334.5 \mathrm{ft} \begin{aligned} & \text { Grab } \\ & \text { Sample }\end{aligned}$ 


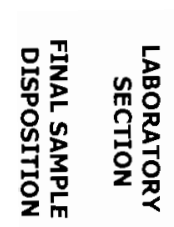
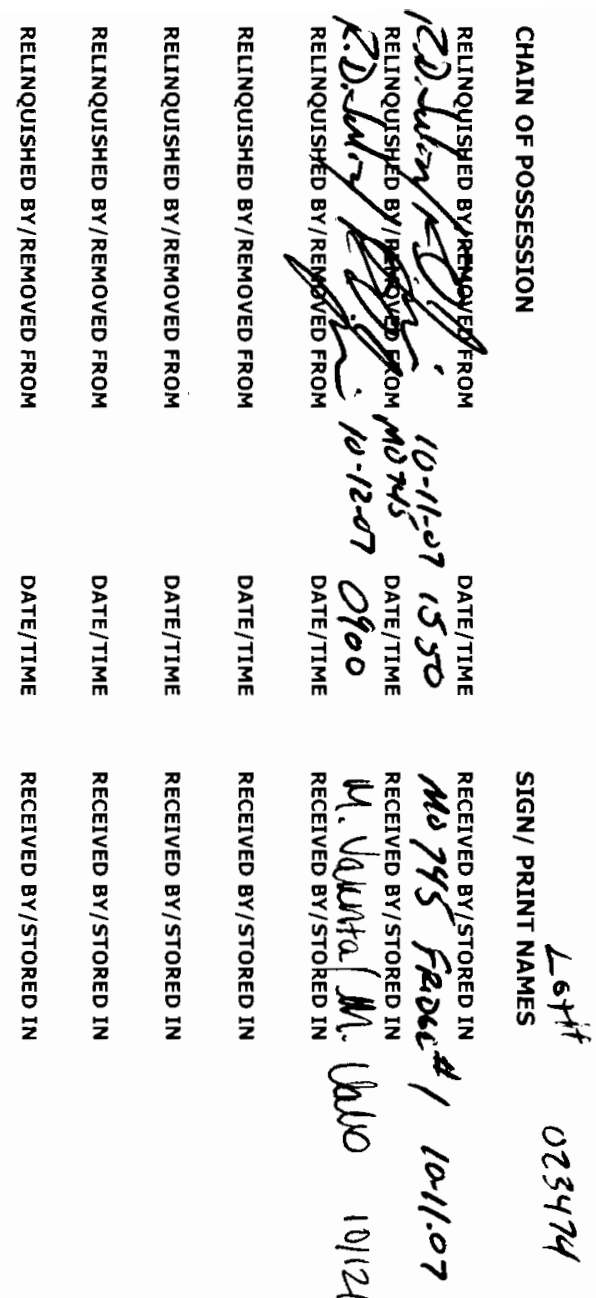

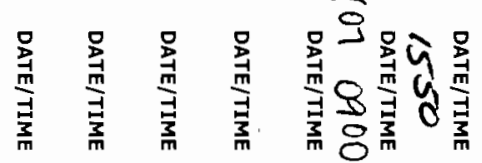

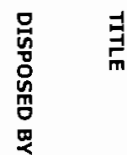

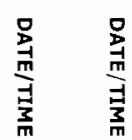

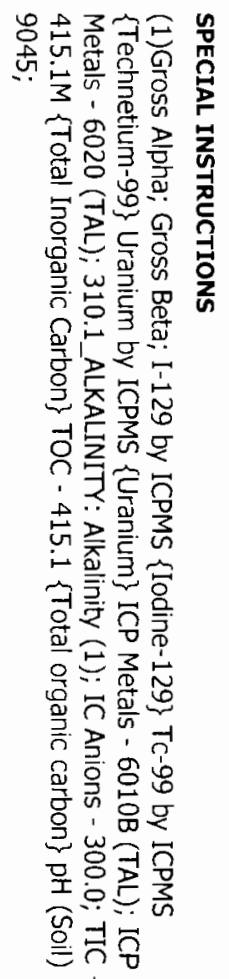

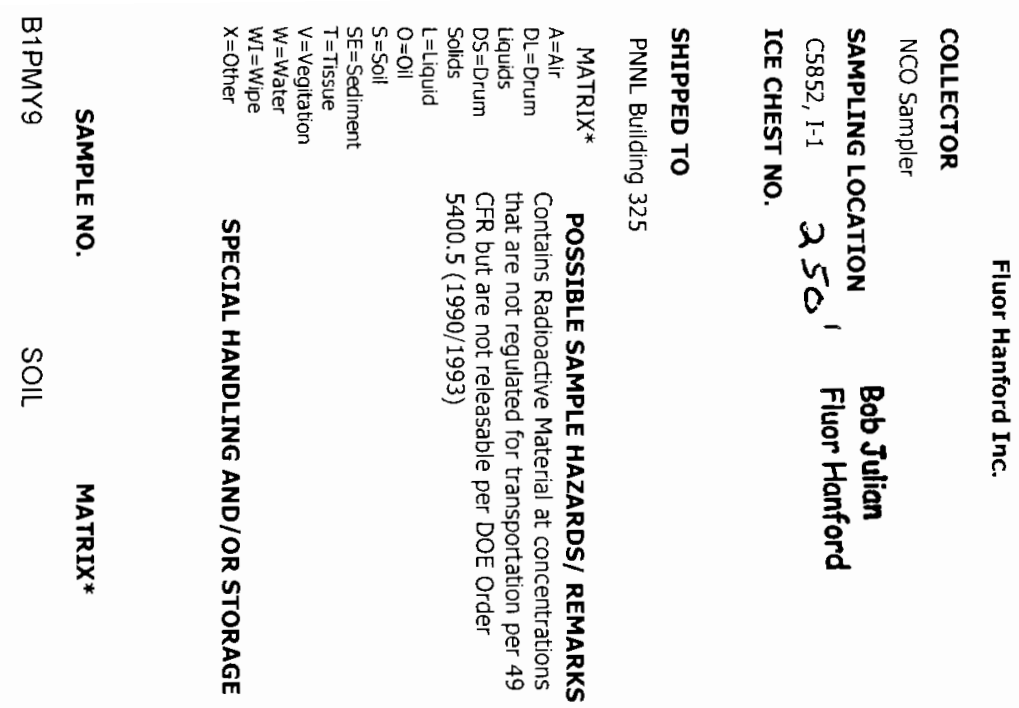

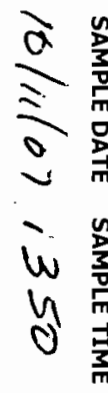

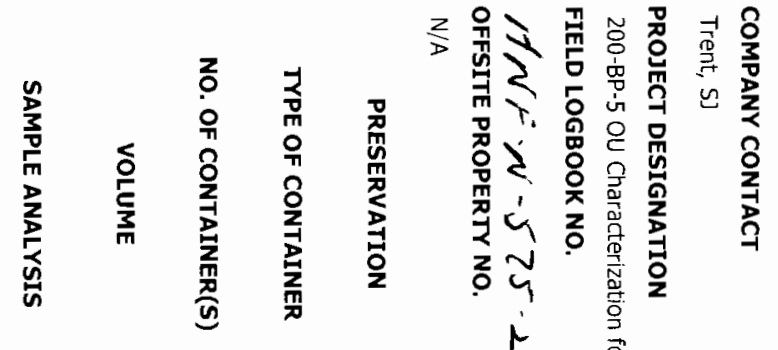

\.

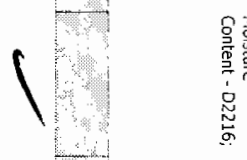

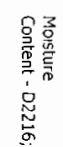

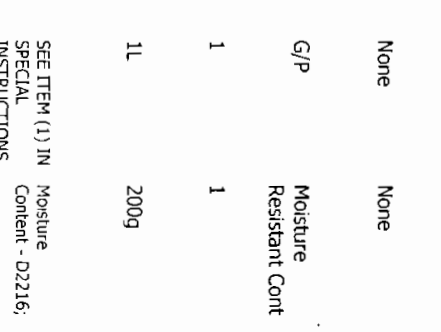




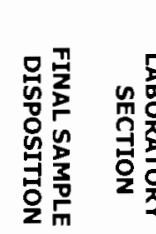

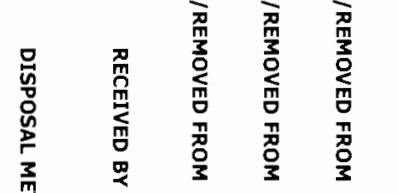

꼼
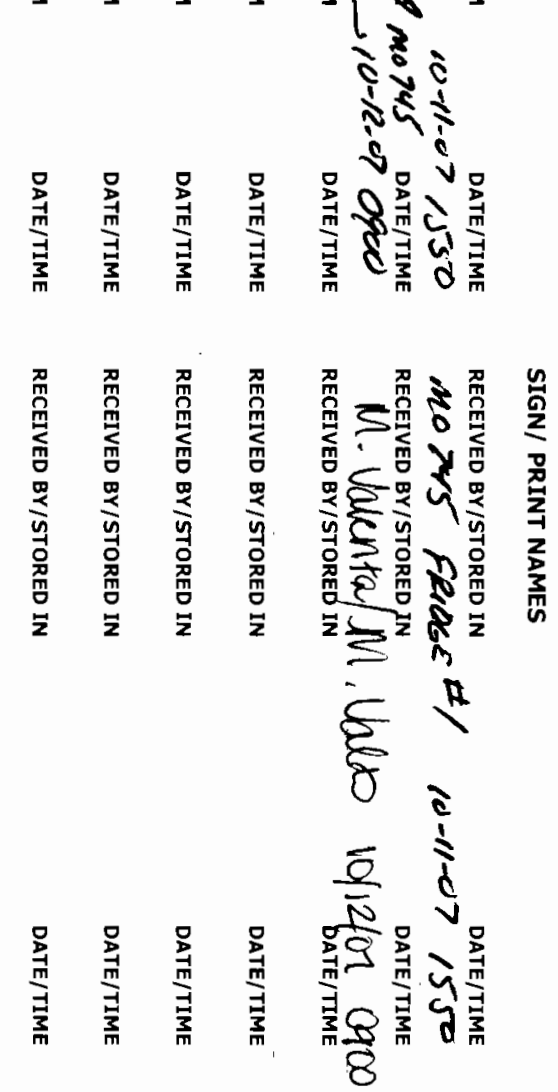

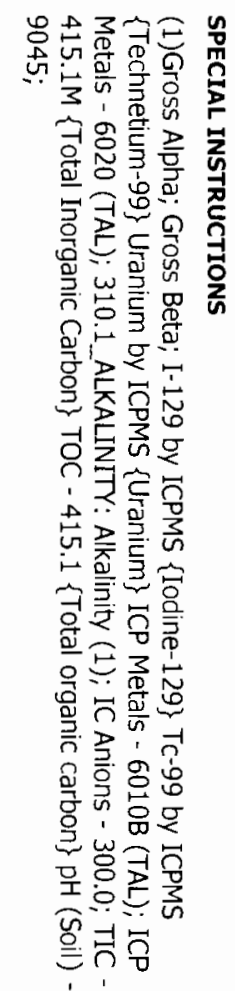

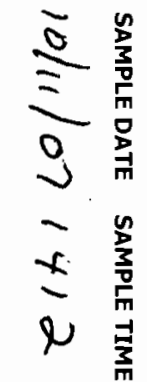

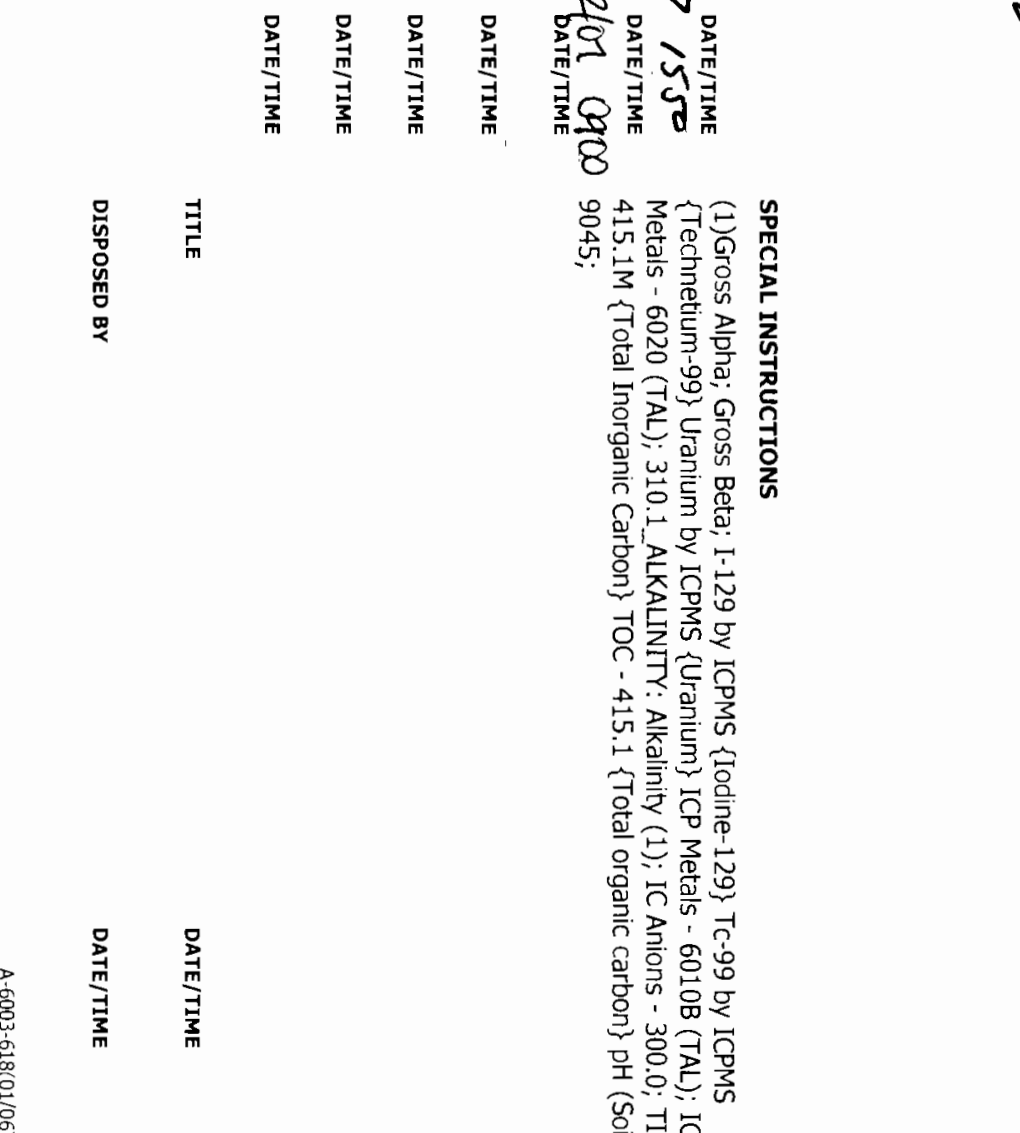
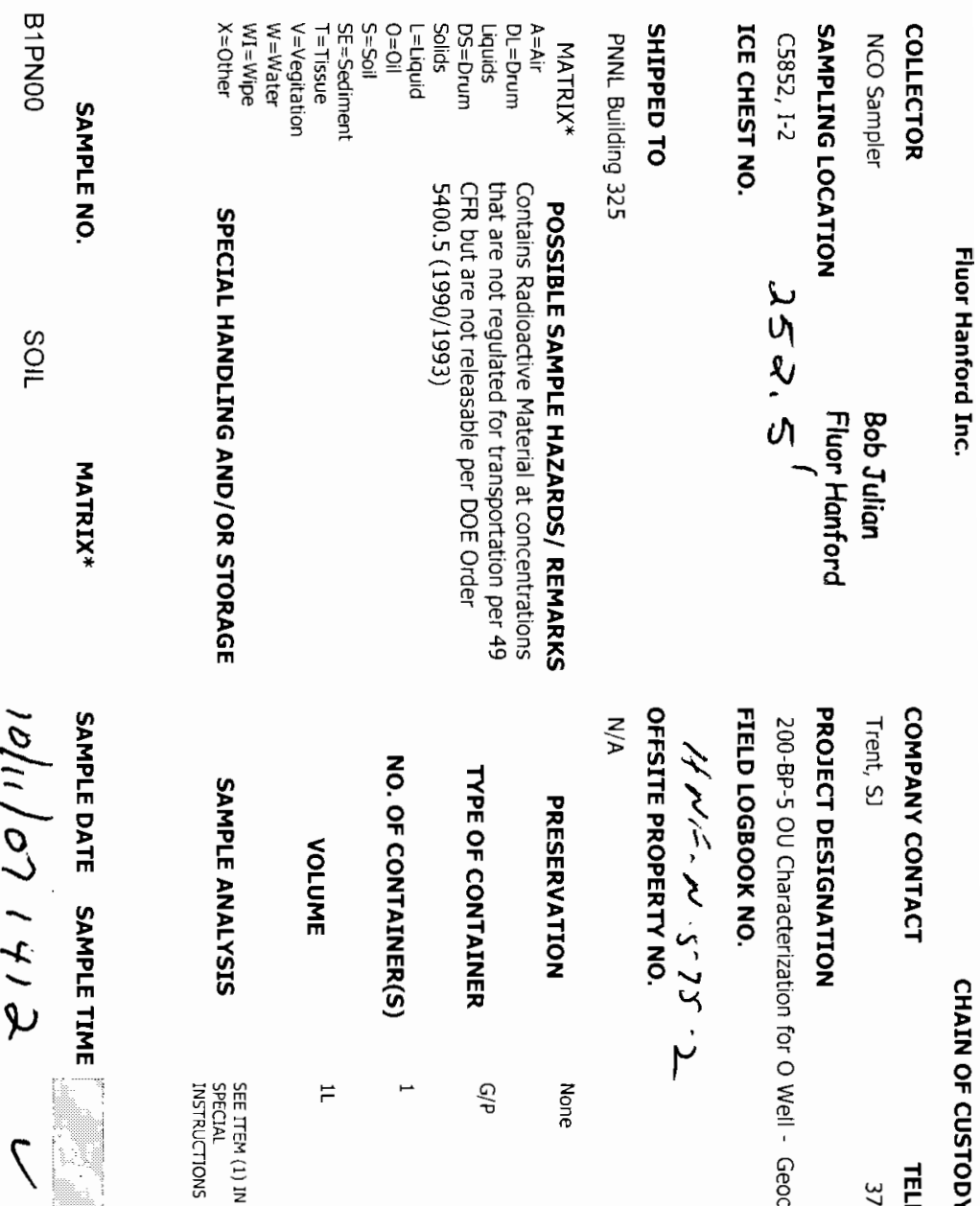

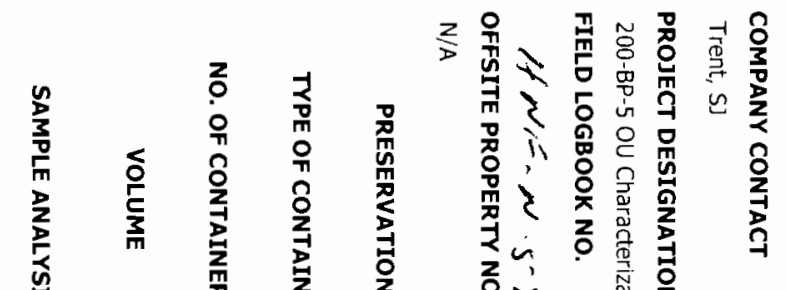

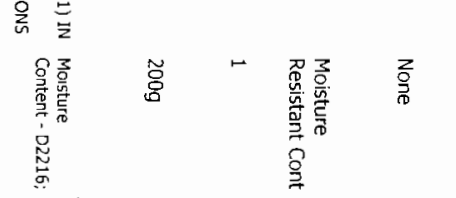


\|\|

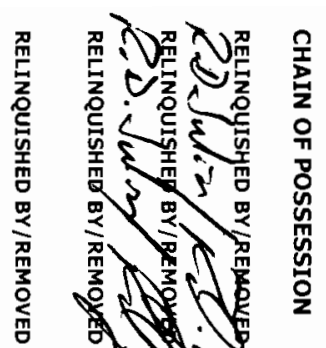

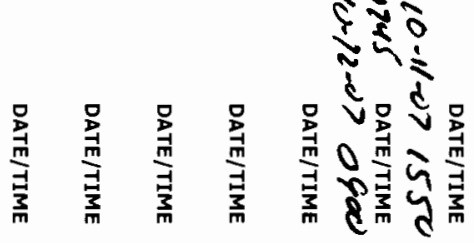
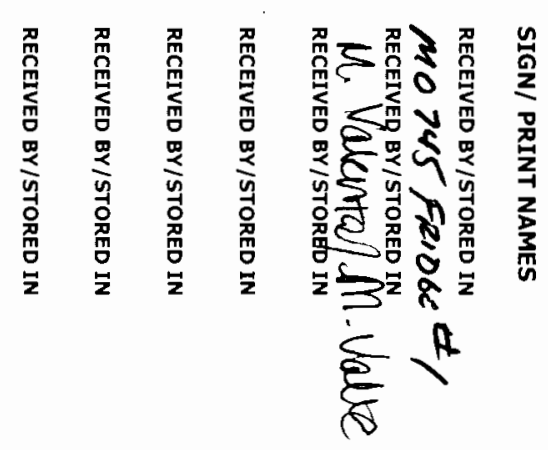

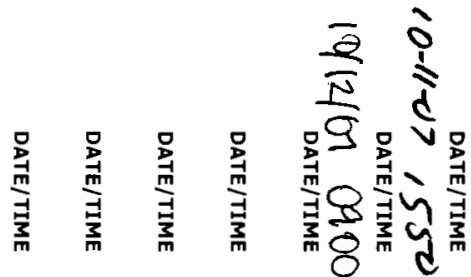

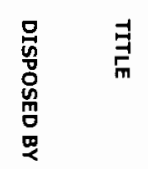

总总

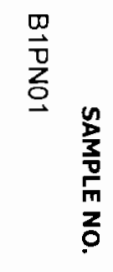

$\stackrel{\text { o }}{=}$

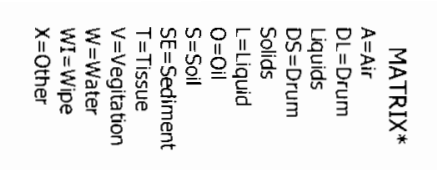

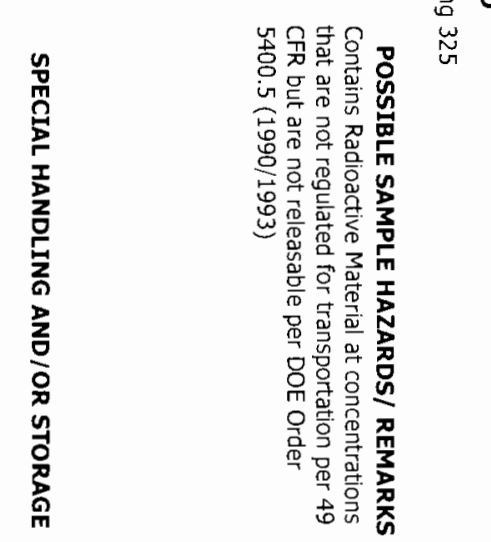

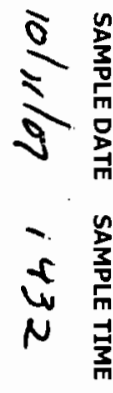

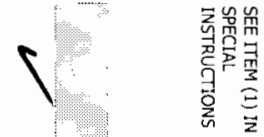

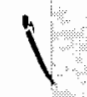

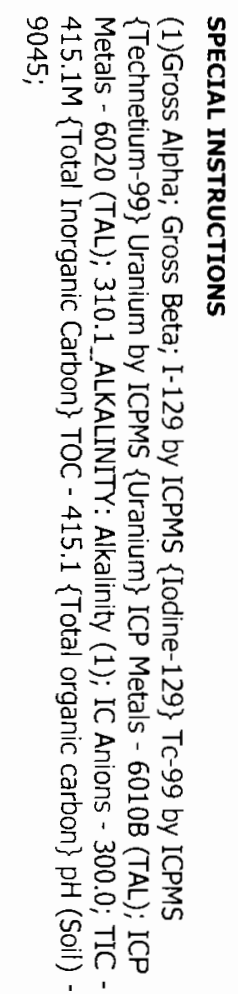

$\underset{*}{\stackrel{3}{7}}$

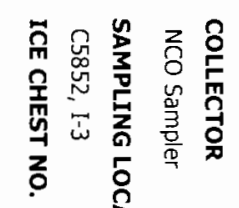

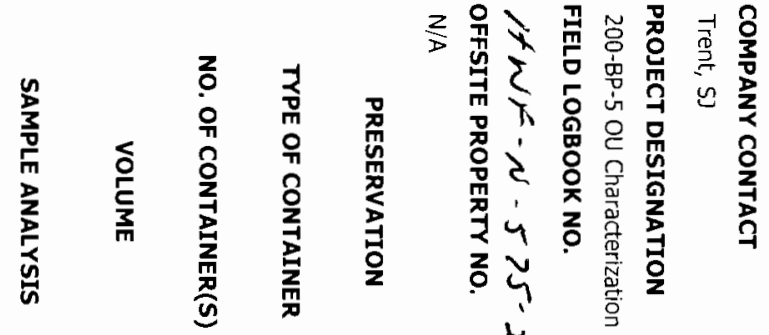

홍

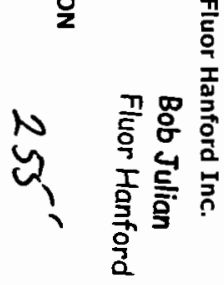

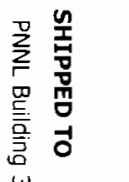<smiles>C1CCCC1</smiles>

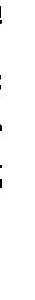




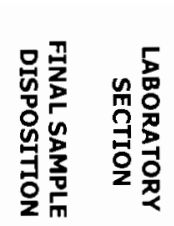

高

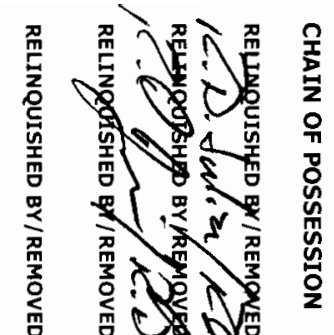

,

टर्ड

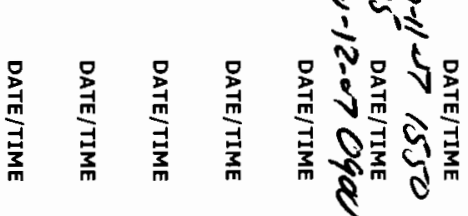

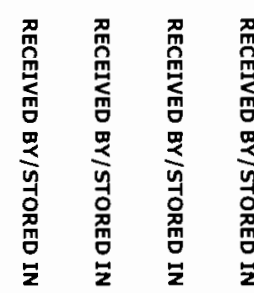

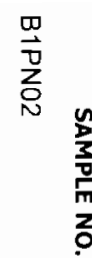

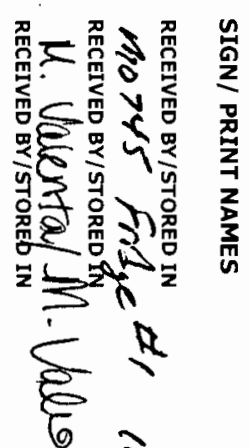

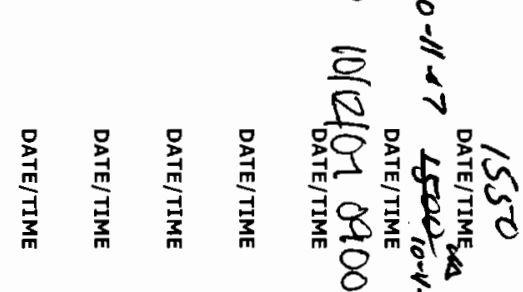

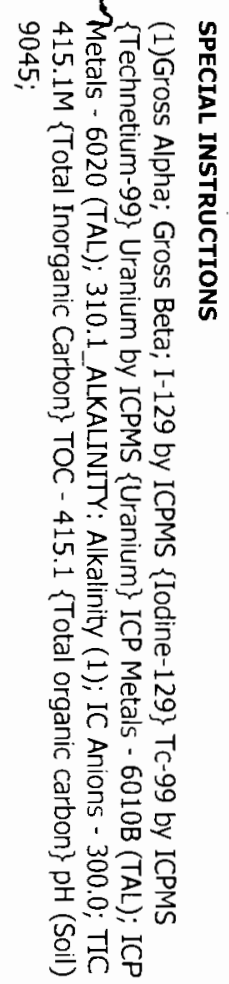

$\stackrel{\infty}{\stackrel{0}{ }}$

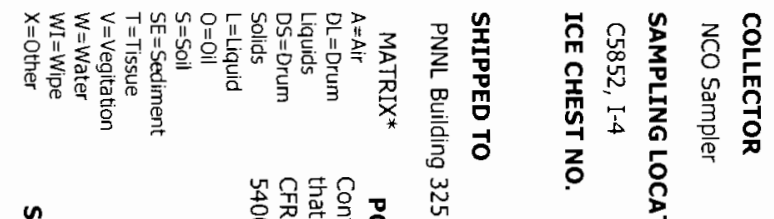

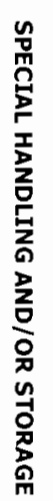

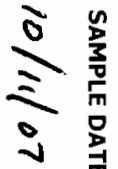

$\begin{array}{ll}\bar{y} & \frac{n}{3} \\ \bar{y} & \frac{5}{7} \\ \frac{3}{3} & \end{array}$

1 高竞
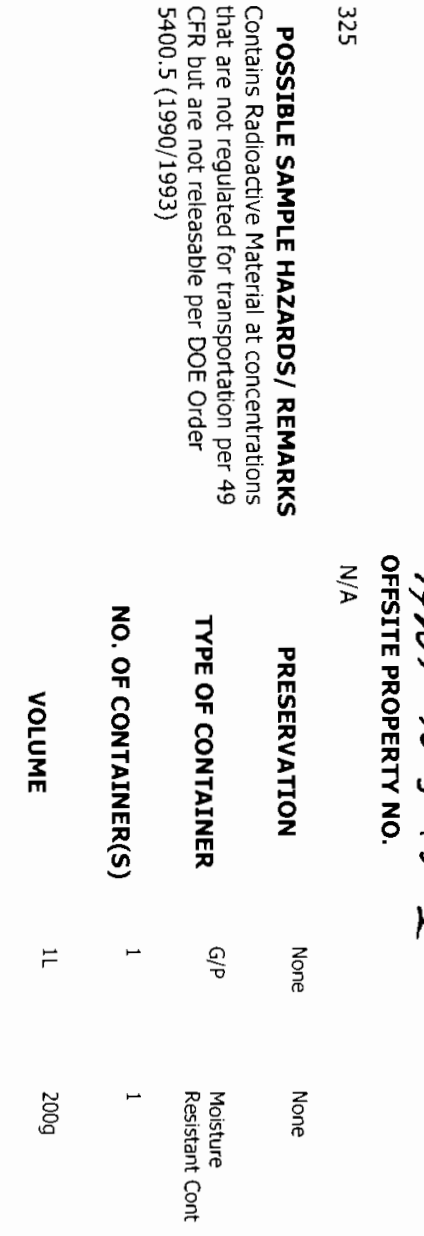

올

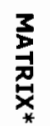

2

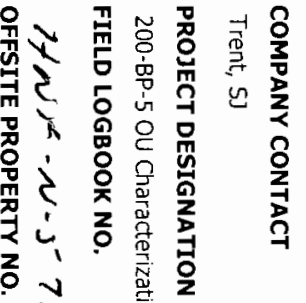

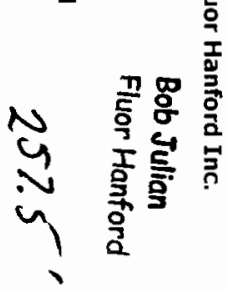

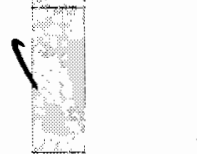<smiles>C1CCCCC1</smiles>
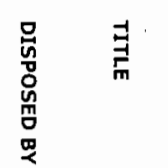

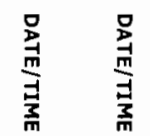

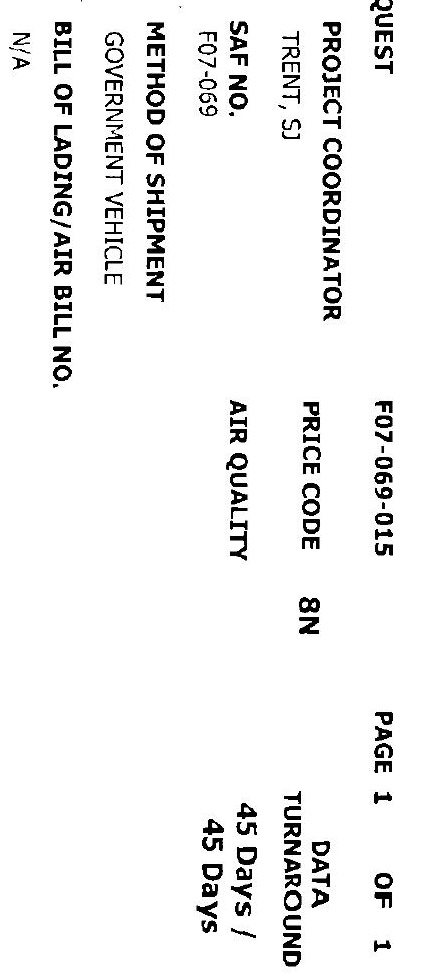




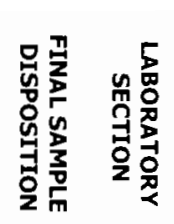

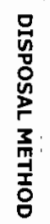

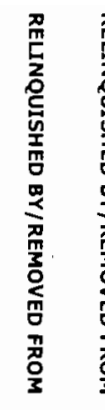
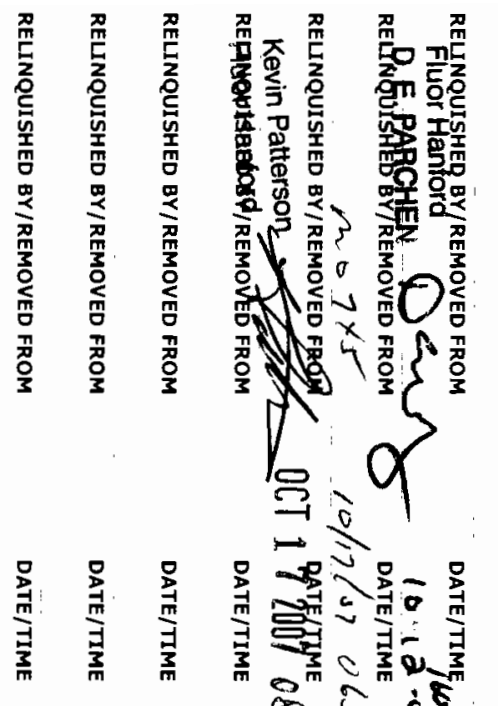

को

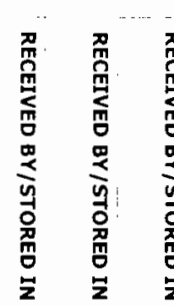

衰
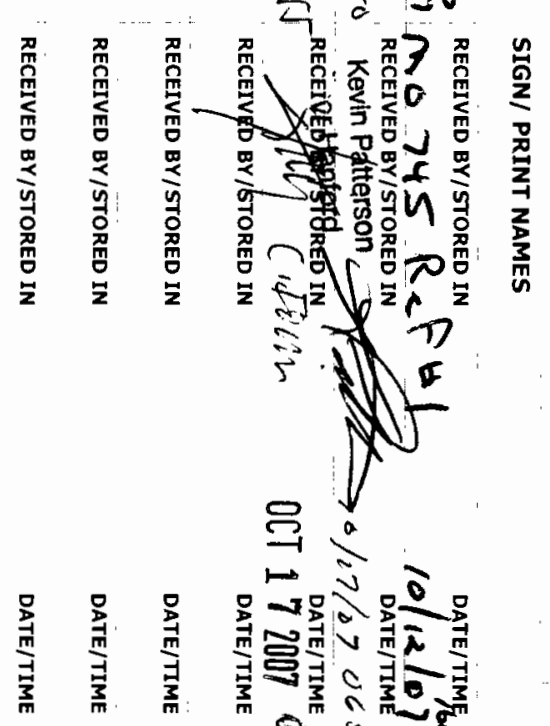

i

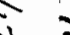

30。

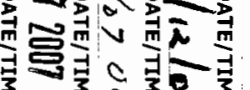

8 造

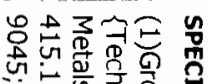

उक के

궁랄

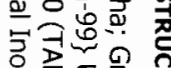

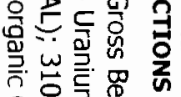

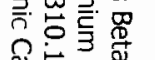

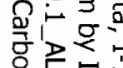

至容它

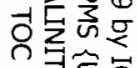

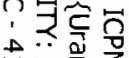

带蛋

刍产总

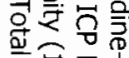

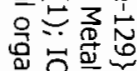

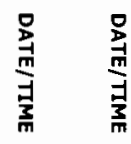

ते ${ }^{-1}$

产院

뭉ㅎㅇ곡

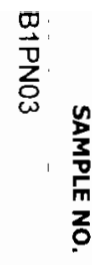

on

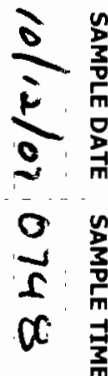

(N)

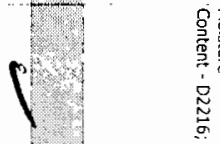

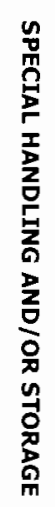

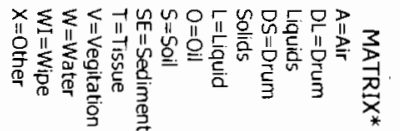

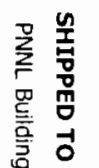

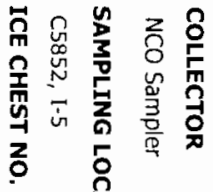

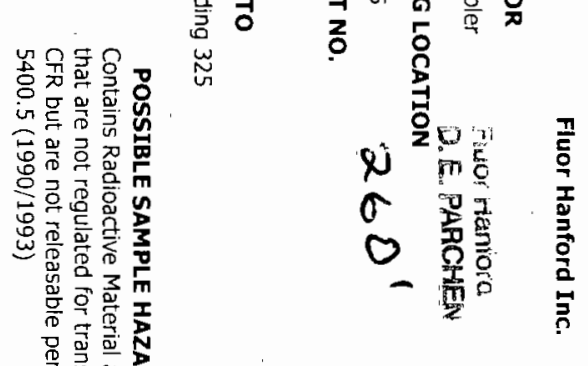

要

鬲举涂

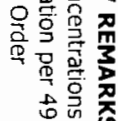

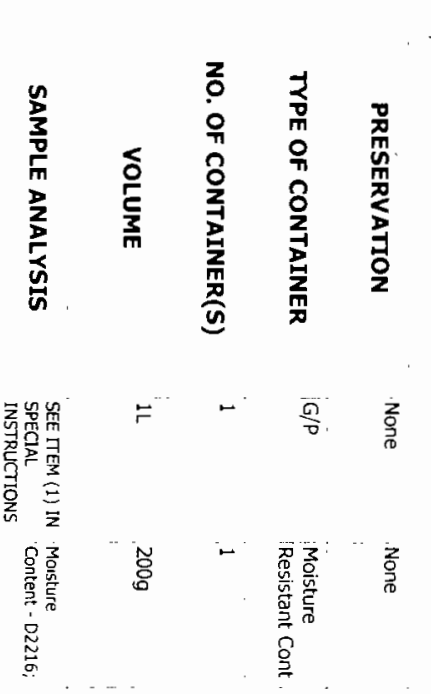

$z$

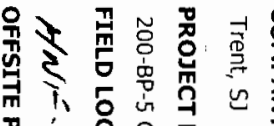

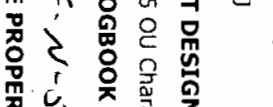

ฟv

人

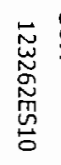

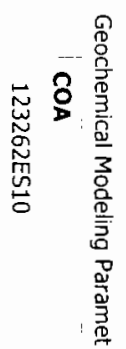

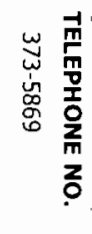

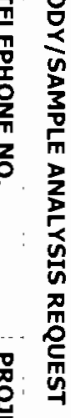

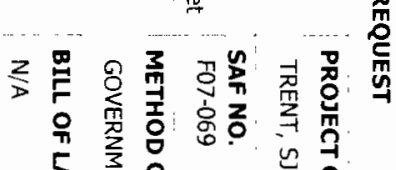

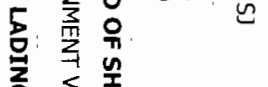

논 孛

吾

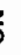

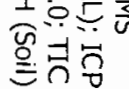




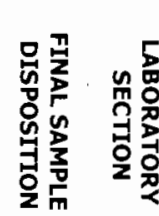
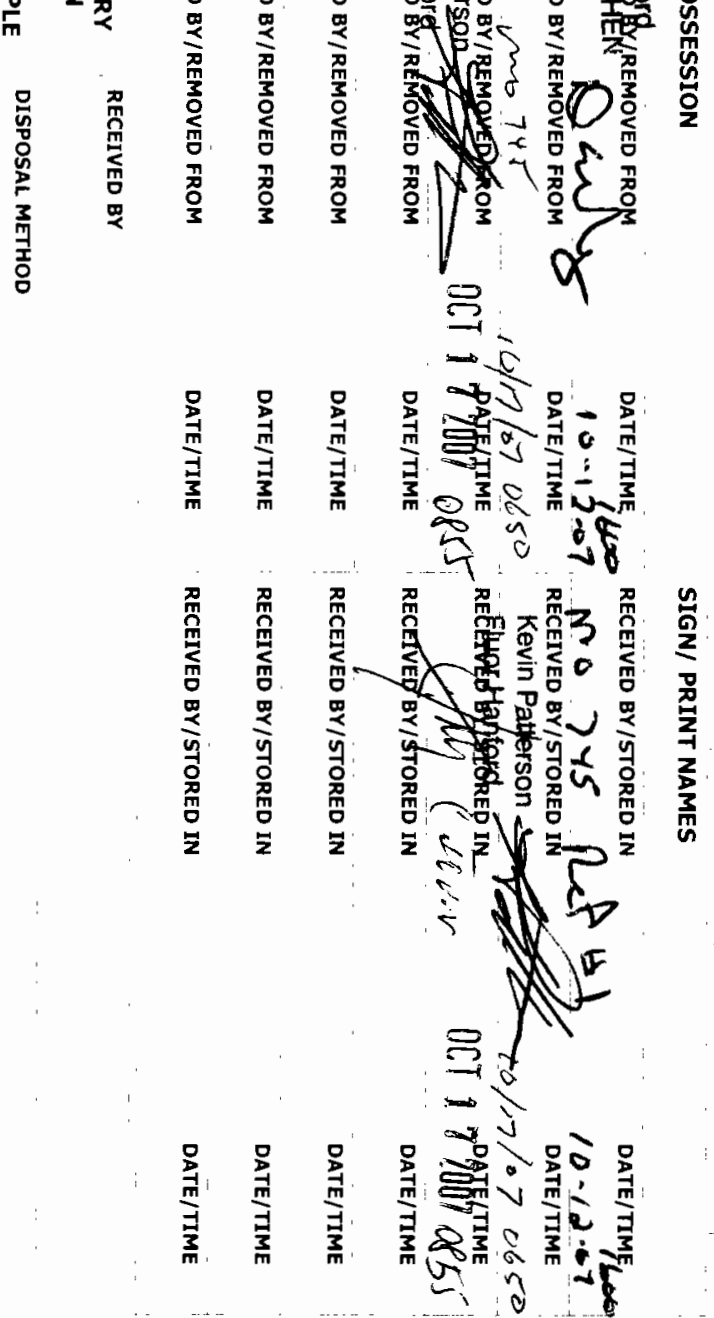

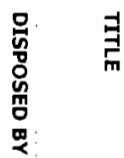

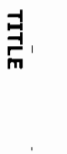

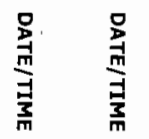

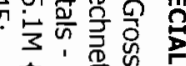
궁류를

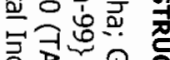

总总高号

言岕焉罾

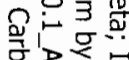

总客完

古害灾产

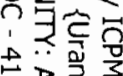

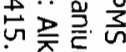

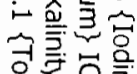

웅ㅎำ

옹 $\frac{3}{0}$

그 ${ }^{-1}$

음 9 ?

금 它产

모영콩뭄

苍뭉
号志要尚已

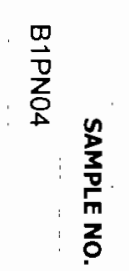

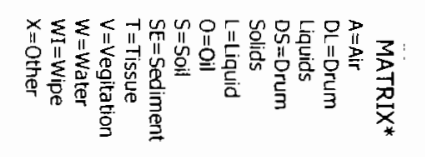

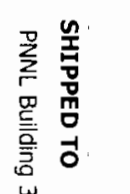

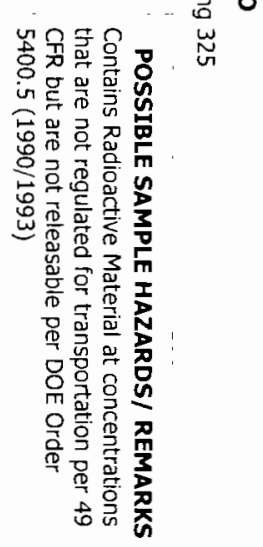

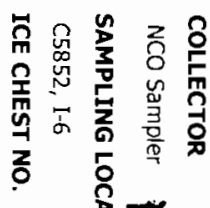

$\stackrel{0}{\circ}$

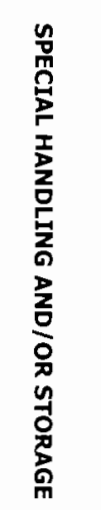

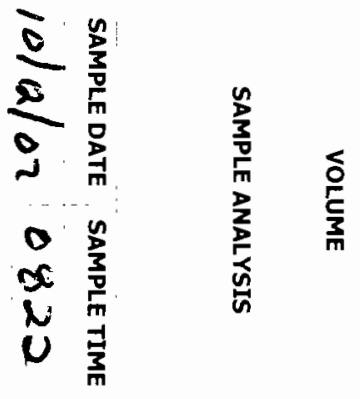

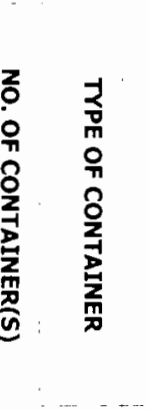

$z$

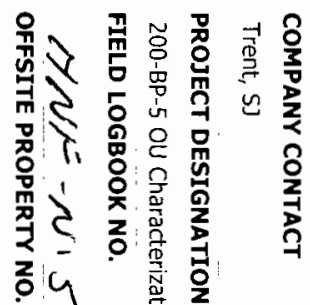

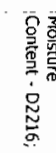

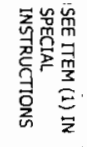 \\ 1}

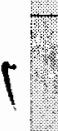

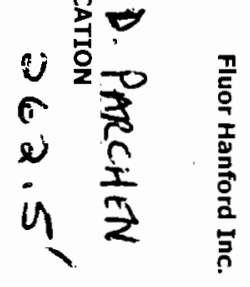



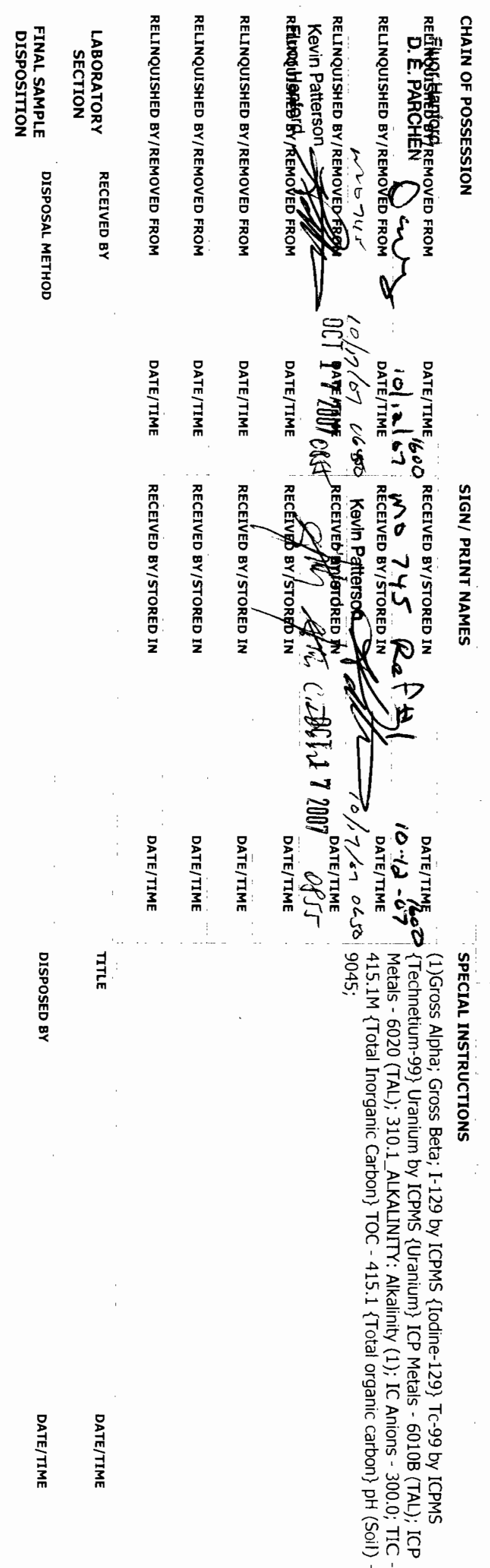

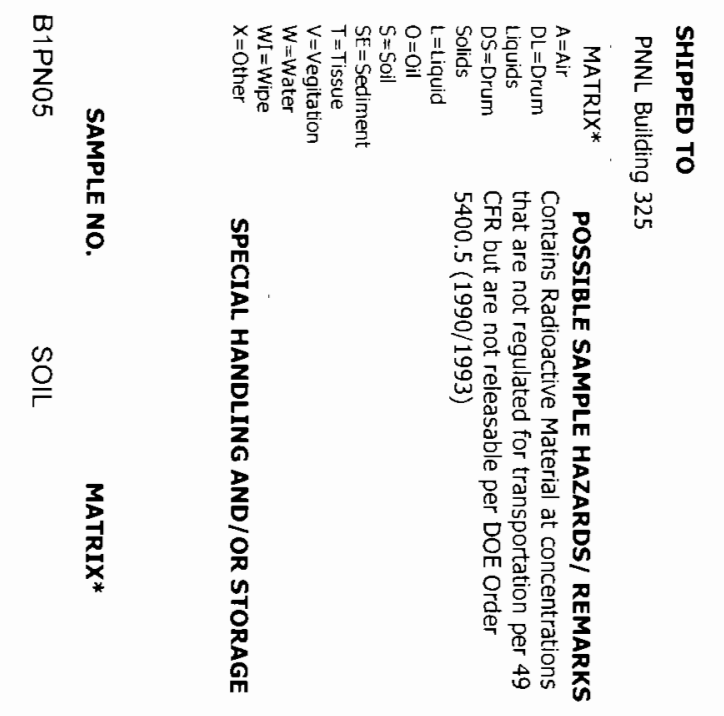

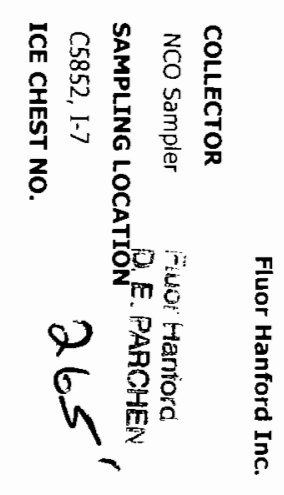

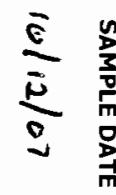

$\frac{1}{2}$

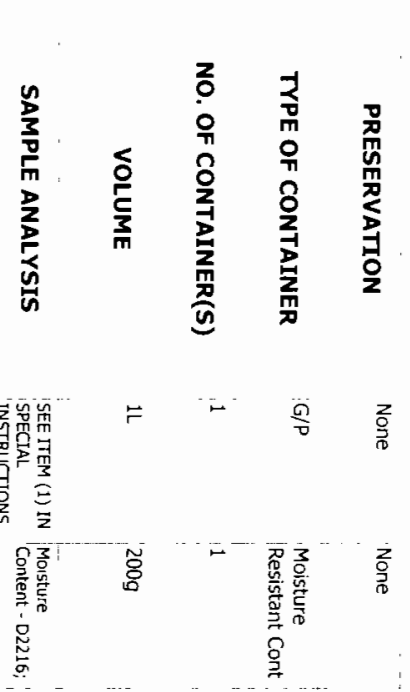

눅 욕

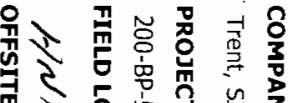

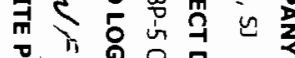

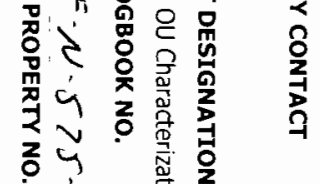

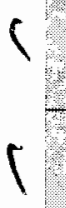

?

$r$

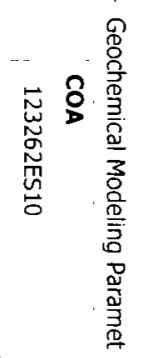

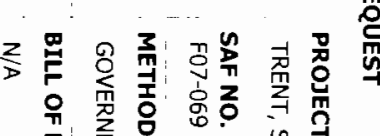

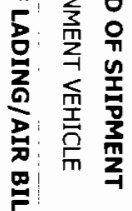

㟧

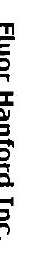

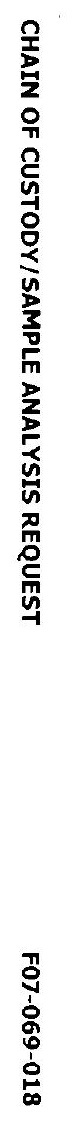
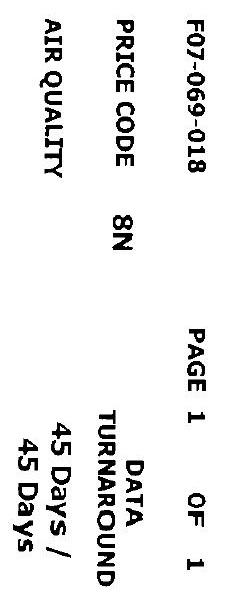

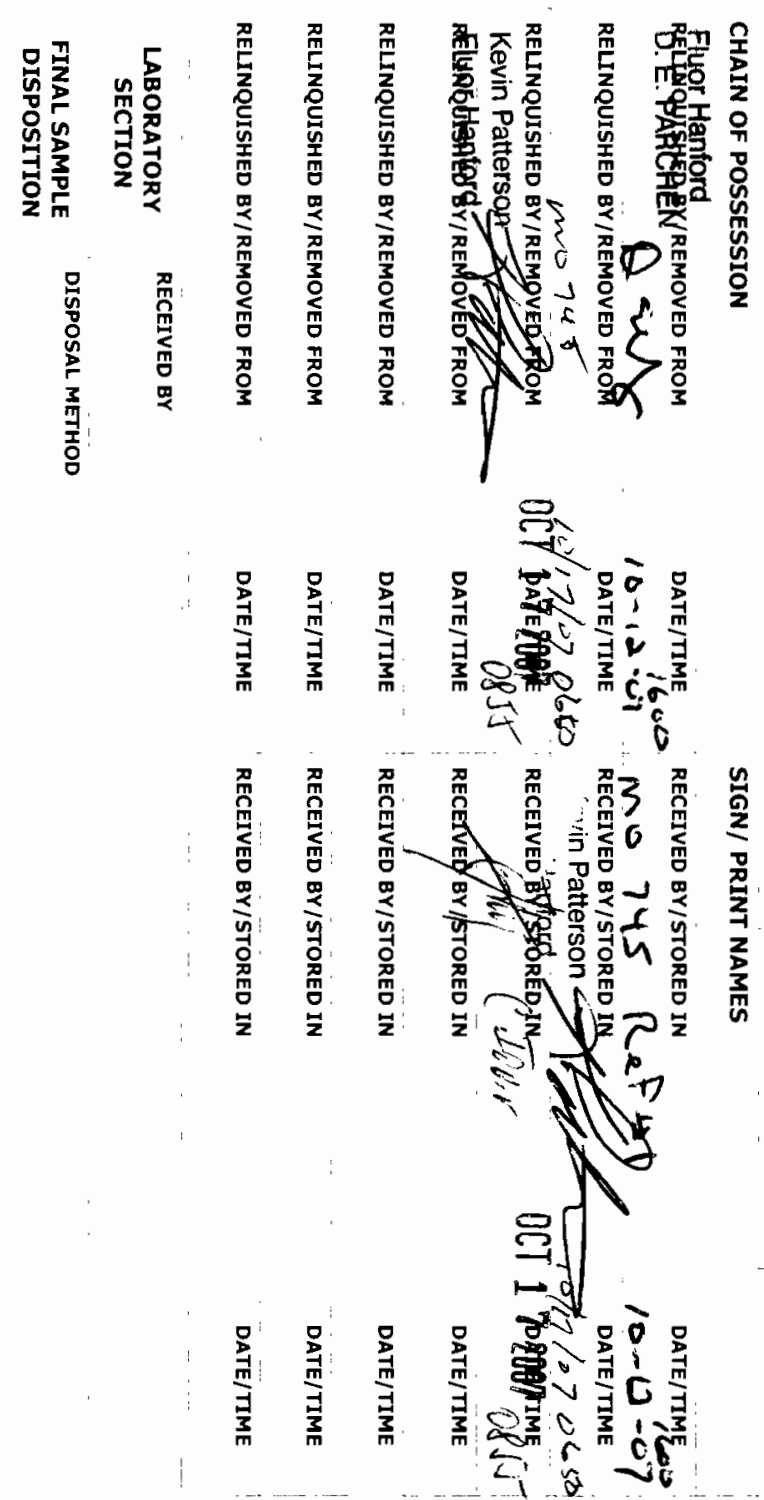

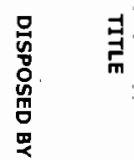

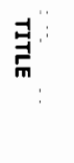

竞
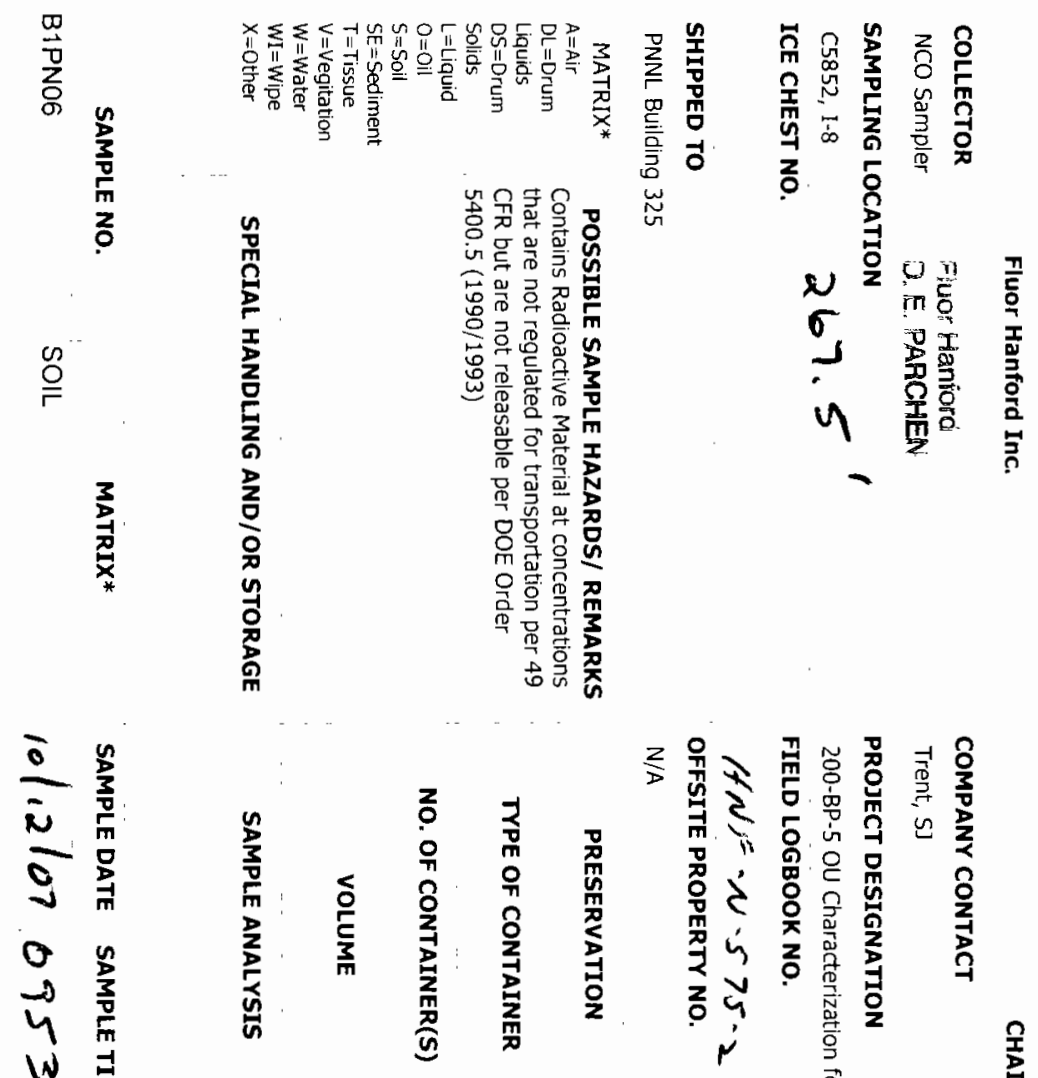

w

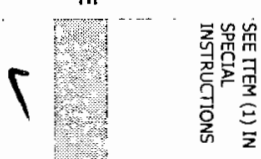

1

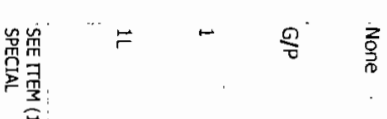

ก 1 -

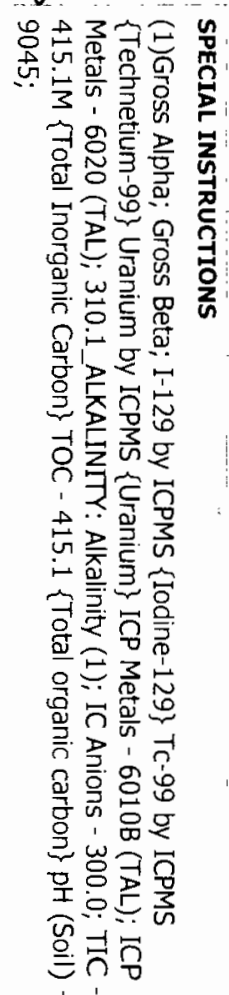

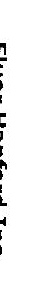



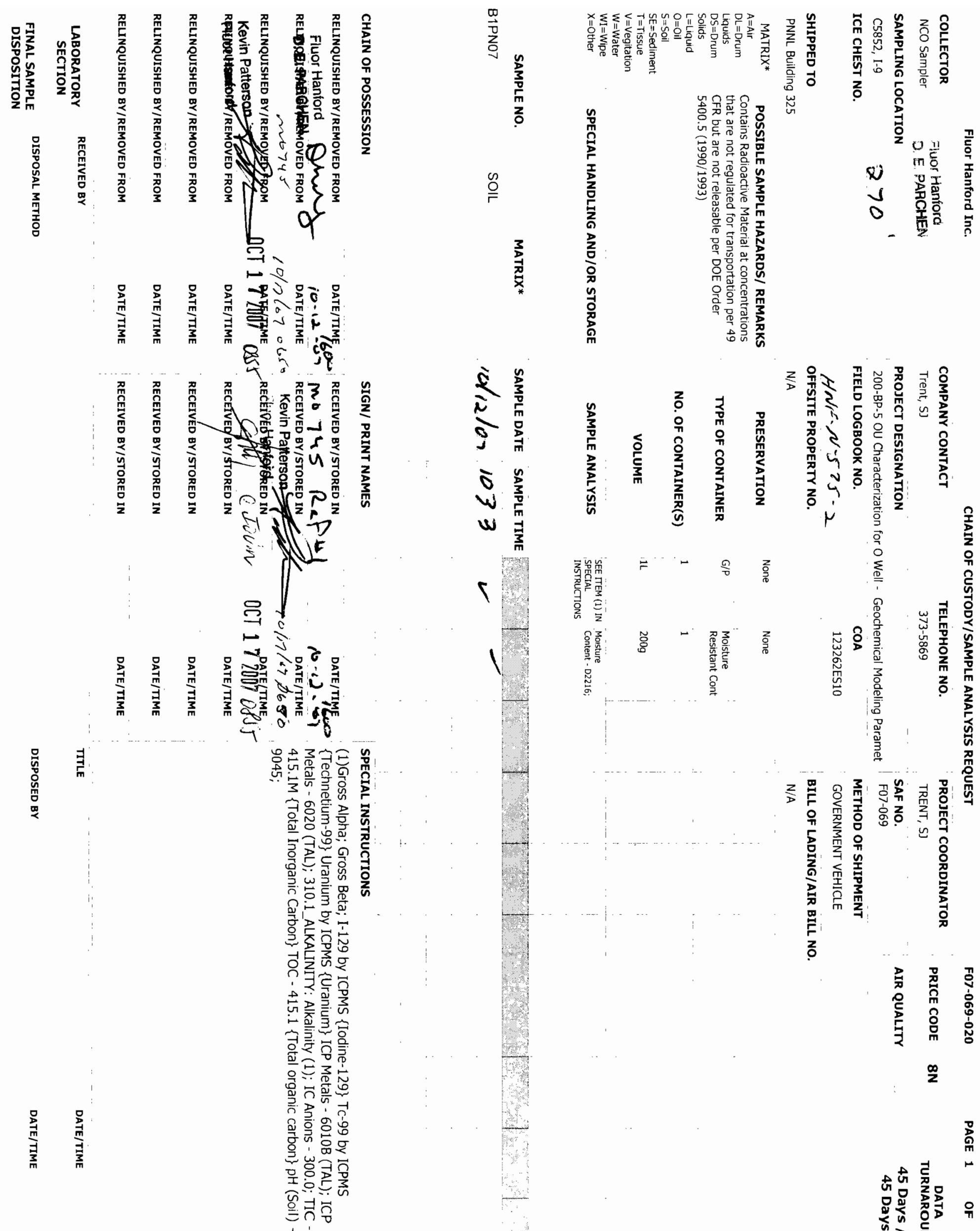

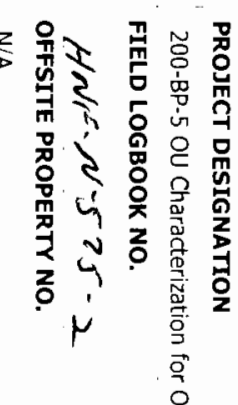
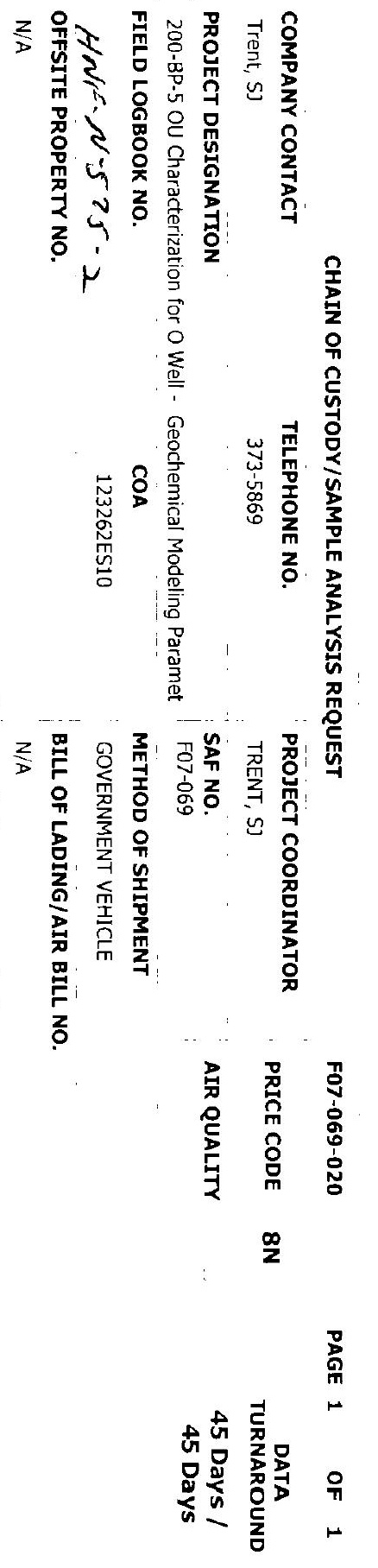


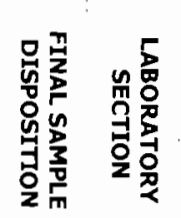

$\frac{n}{11}$

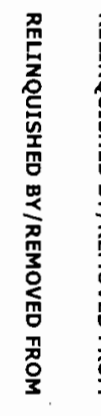
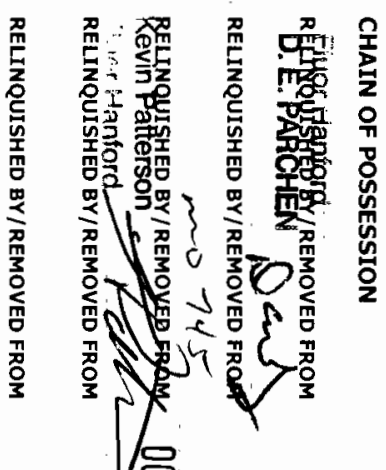

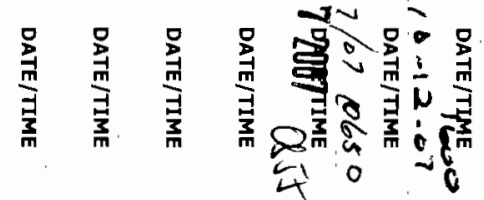

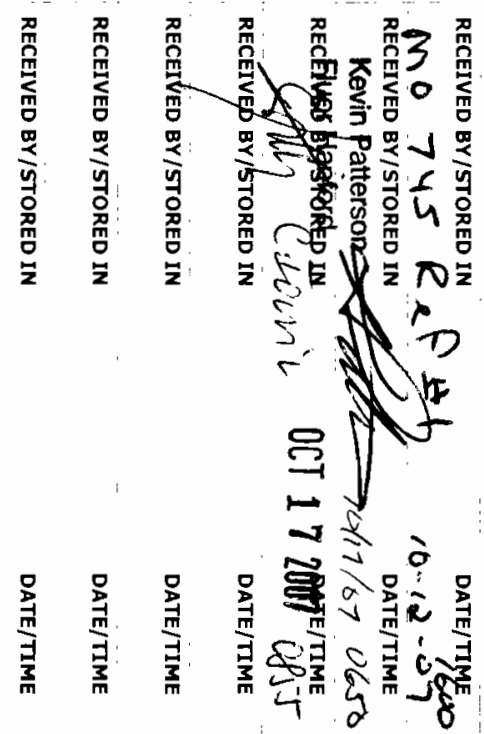

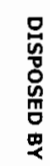

:

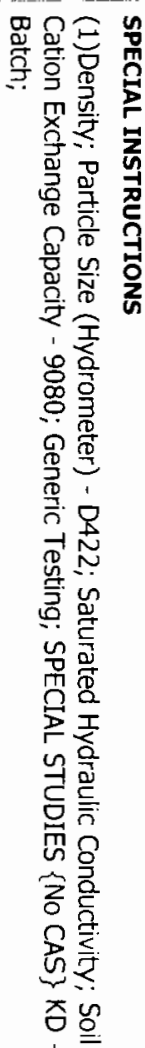

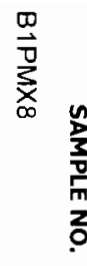

$\stackrel{\stackrel{0}{O}}{\Gamma}$

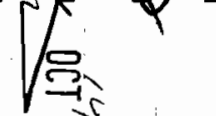

$\stackrel{\infty}{=}$

$\frac{\bar{p}}{i}$
$\dot{j}$
$\bar{j}$

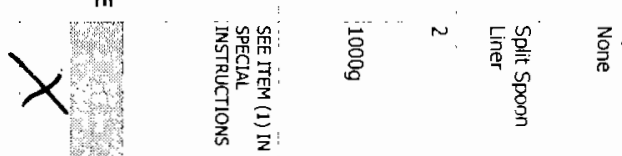

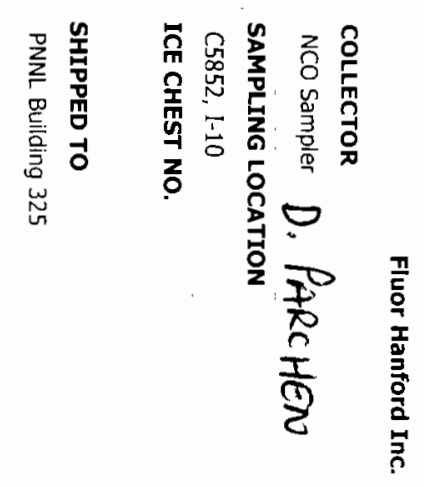

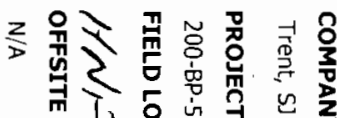

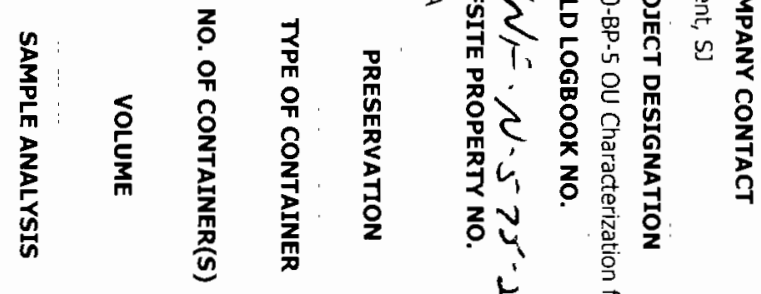

울

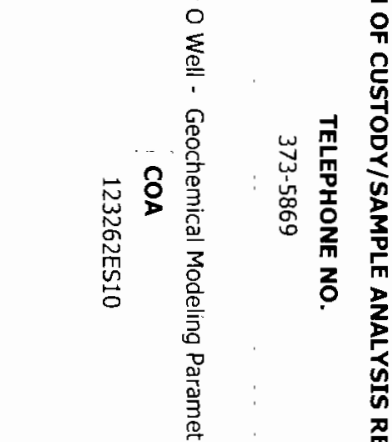

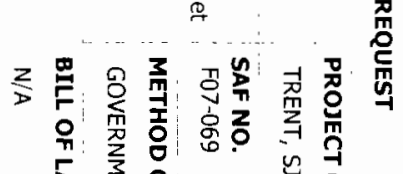

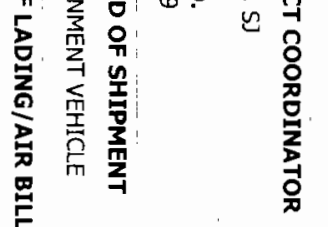

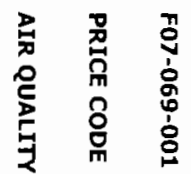

$\frac{\infty}{2}$

章

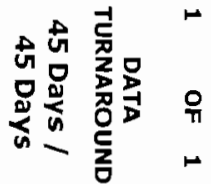




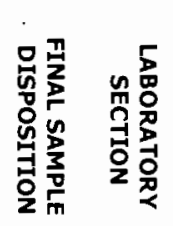

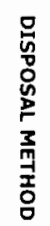

ख
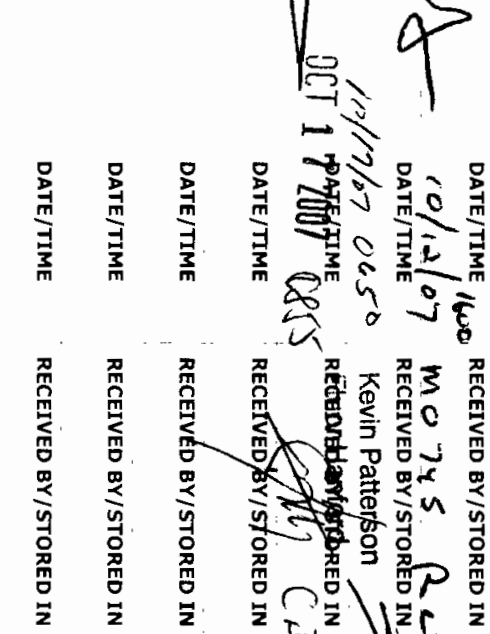

प.

.

\section{音}

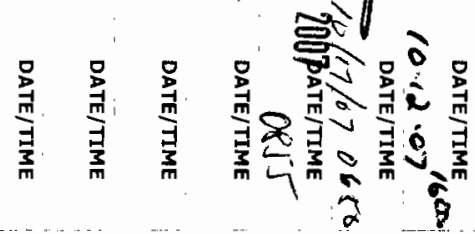

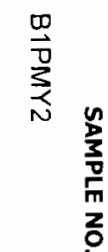

$\stackrel{\infty}{\stackrel{\rho}{ }}$

$\underset{3}{\frac{3}{3}}$

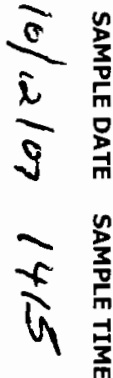

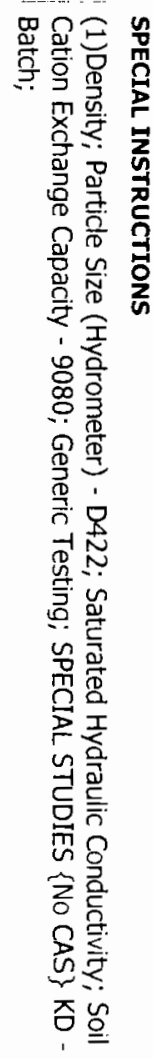

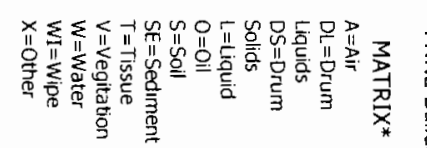

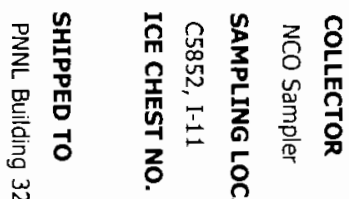

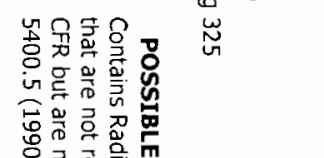

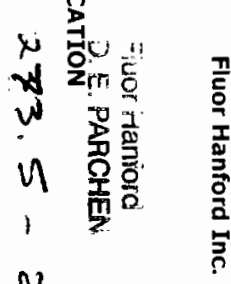

p

牙

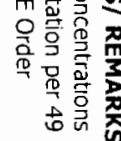

6

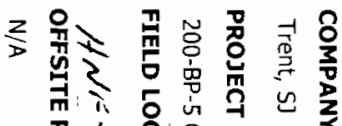

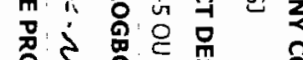

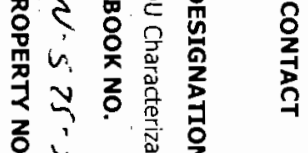

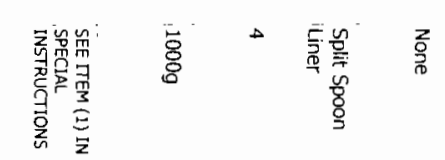

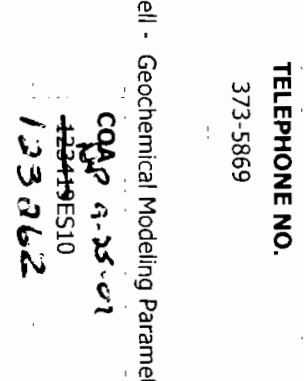

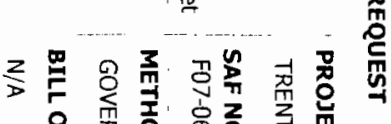

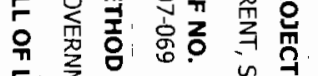

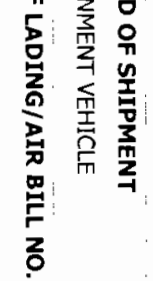

总

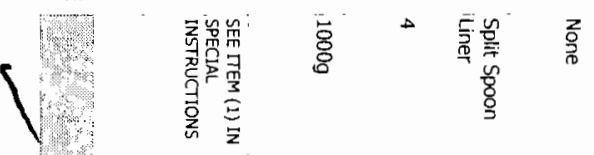




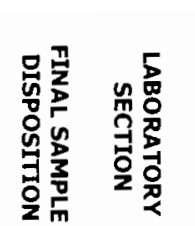

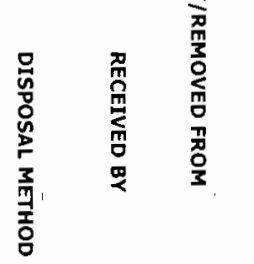

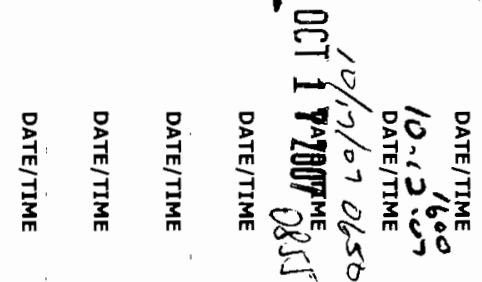

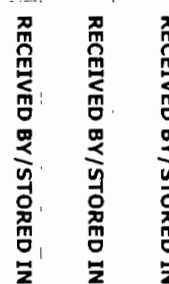
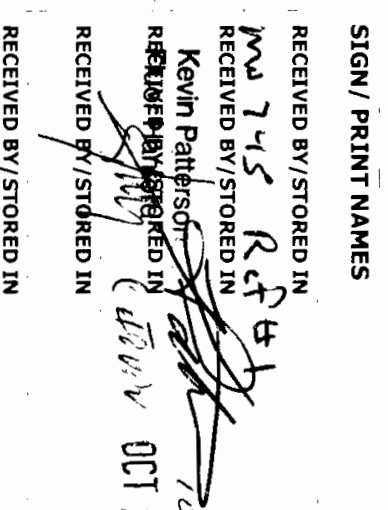

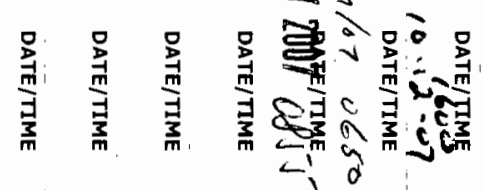

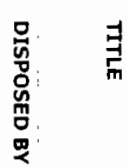

$\begin{array}{ll}\frac{9}{3} & \frac{0}{3} \\ \frac{7}{3} & \frac{7}{3} \\ \frac{3}{3} & \frac{3}{3}\end{array}$

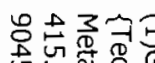

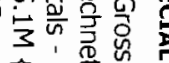

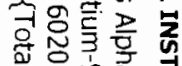

ت3总总

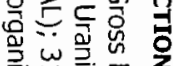

言岩穿思

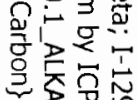

需勇它

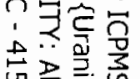

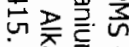

当产告高

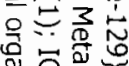

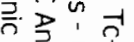

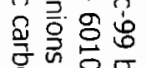

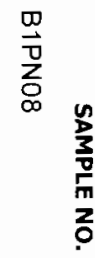

$\stackrel{\oiiint}{\rightleftharpoons}$

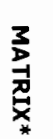

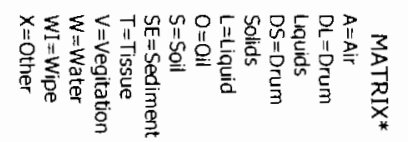

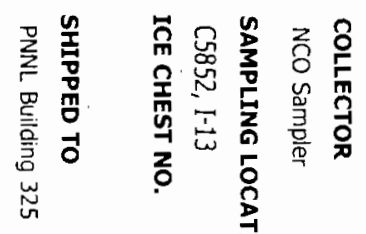

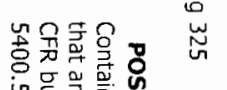

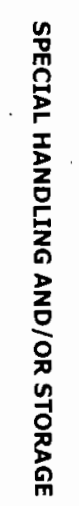

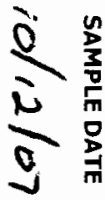

w

U 국

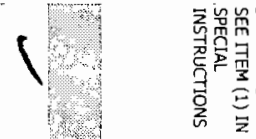

1

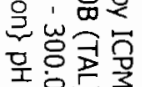

至武它

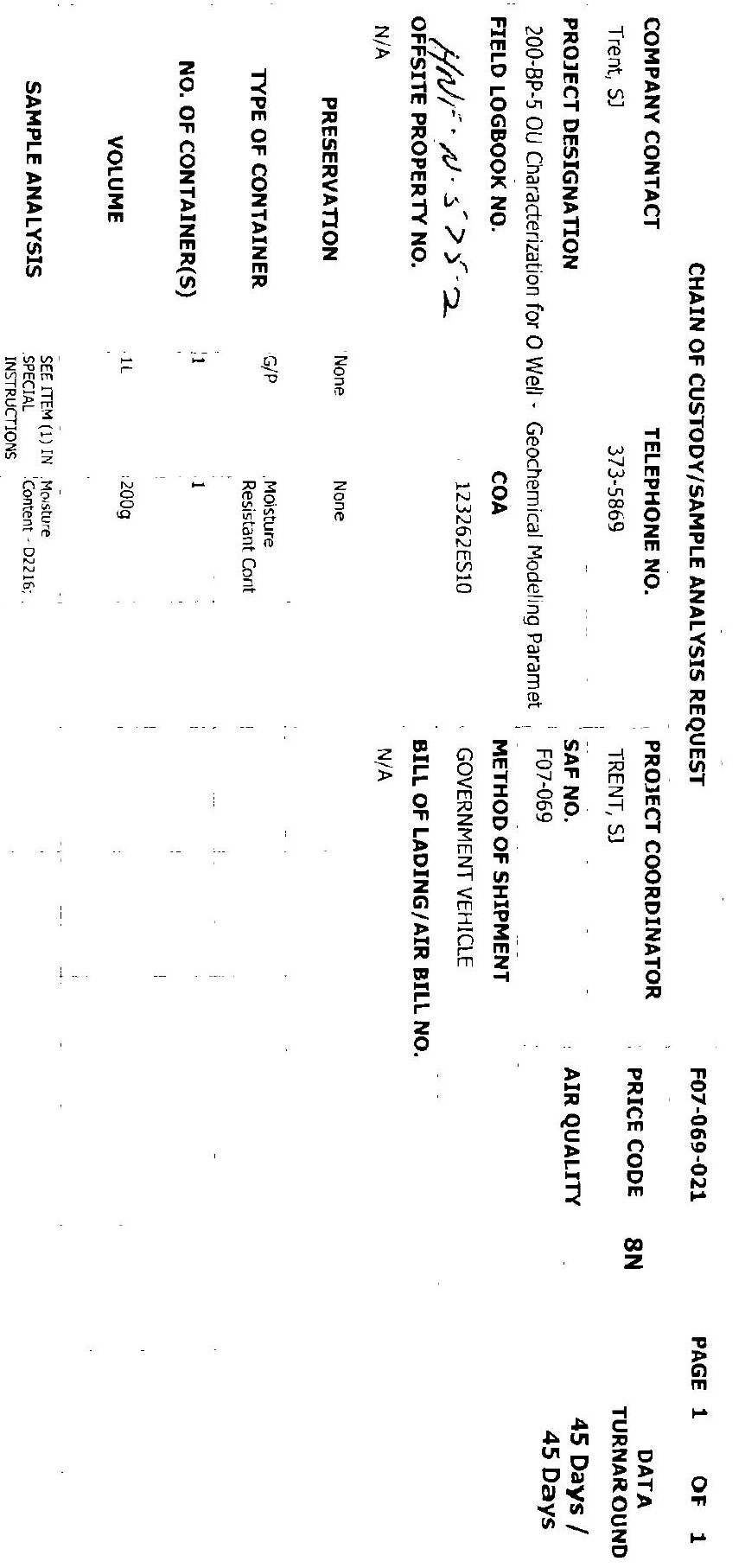




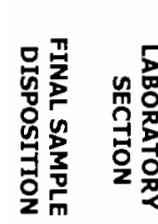

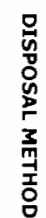

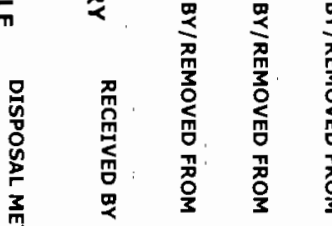

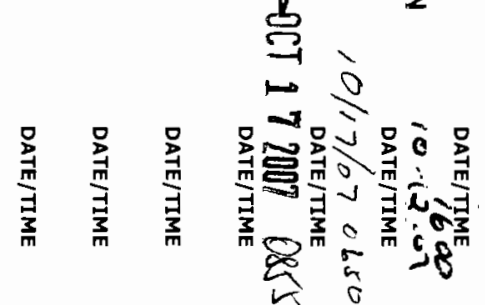

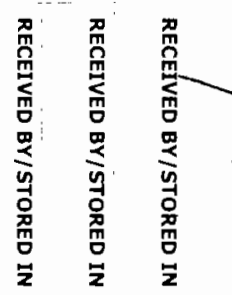

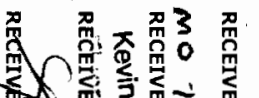

2.

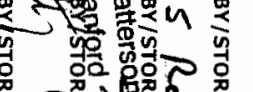

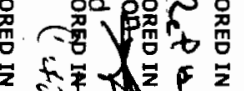

:

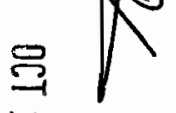

$\rightarrow 2$

웅

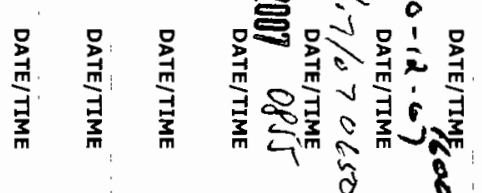

莺 劣

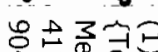

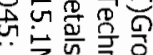

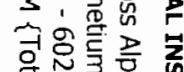

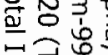

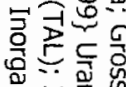

卷岩总罾

Q는

突㲾它

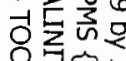

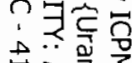

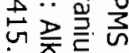

该

을

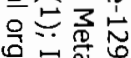

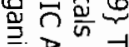

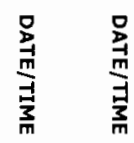

จ흥ㅇㅇ

늘 $\underset{\overrightarrow{0}}{\stackrel{0}{0}}$

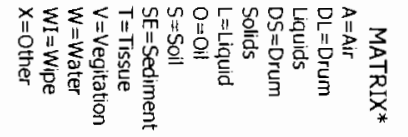

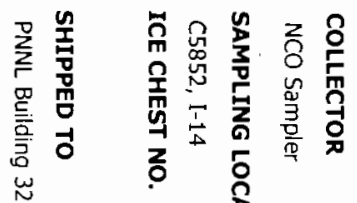

$\stackrel{90}{\stackrel{2}{*}}$

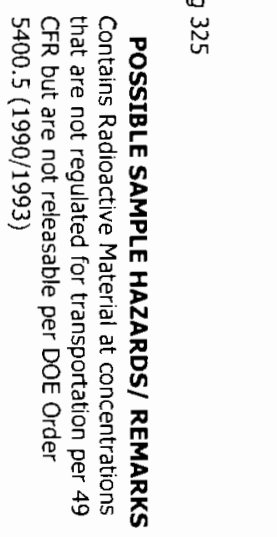

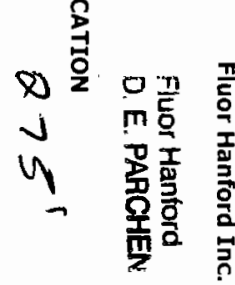

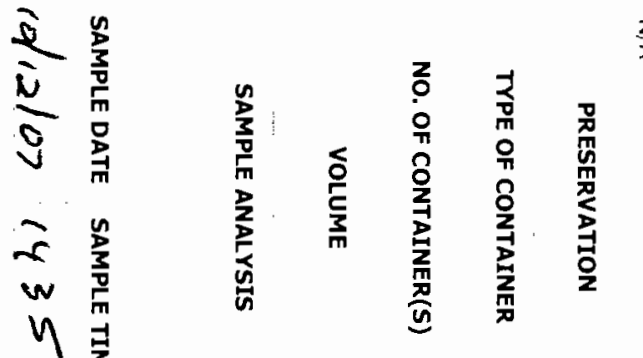

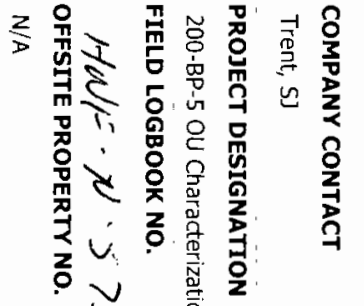

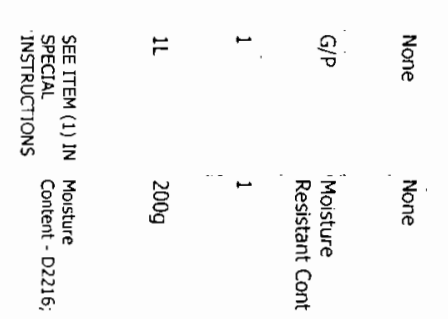

뭉ㅇㅇ고웜

包尓官 


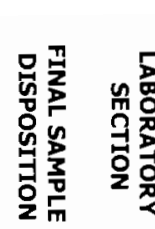

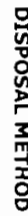
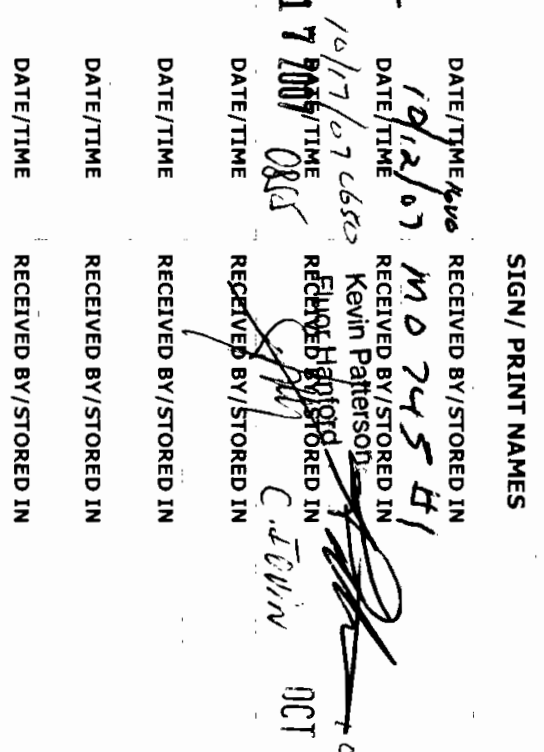

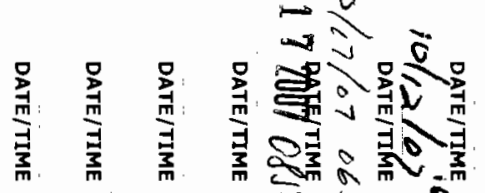

\section{$\underset{m}{\exists}$}

$4 y$ है.

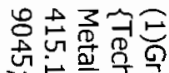

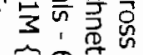

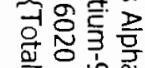

콜웜

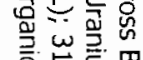

施罗

Q

恶至完

이을

글옹

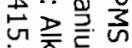

访突灵

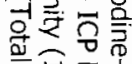

司息

岂 $\frac{\omega}{n}$

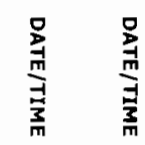

فे

ज列

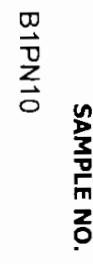

$\stackrel{\mathscr{O}}{\rightleftharpoons}$

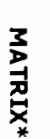

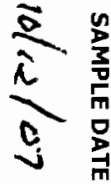

$\begin{array}{ll}4 & \frac{n}{3} \\ 0 & \frac{m}{5} \\ 0 & \frac{\vec{n}}{3}\end{array}$

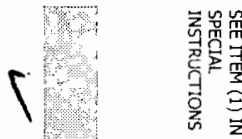

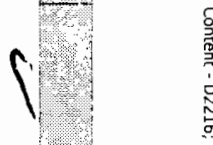

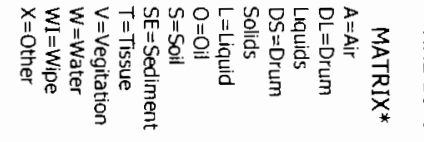

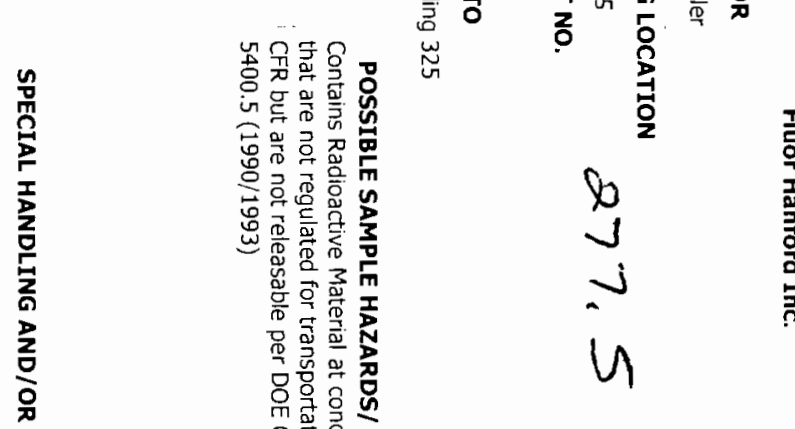

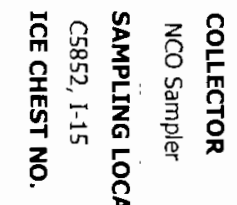

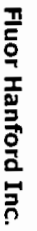

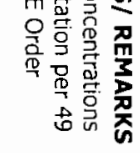

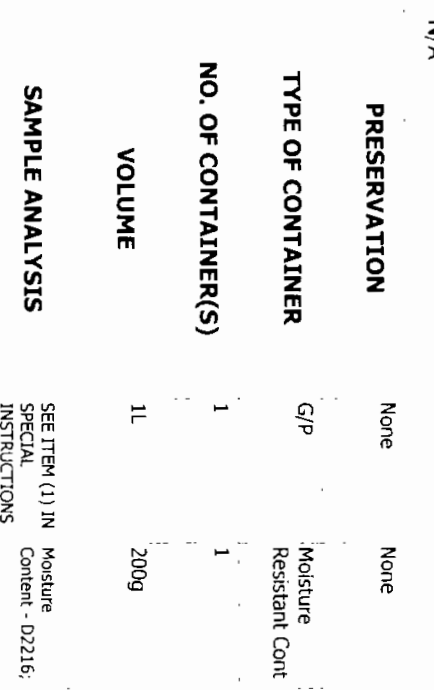

$z \frac{\text { 귝 }}{7}$

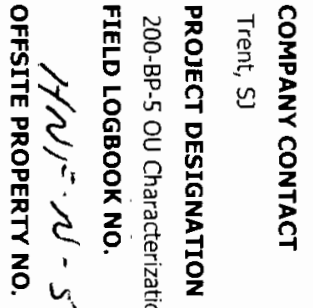

올 ㄴ,

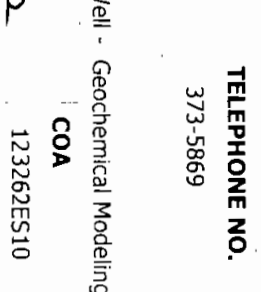

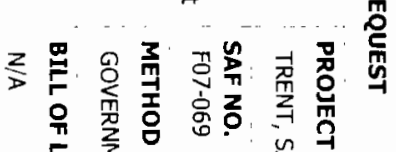

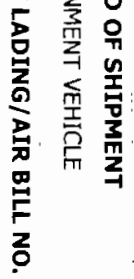

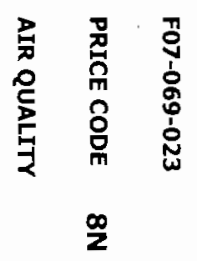

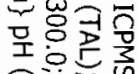

응ㅁํㅇ 


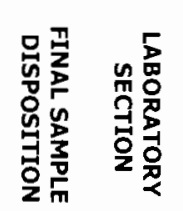 \\ 목 \\ २}

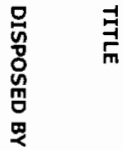

是

훙호를

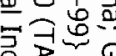

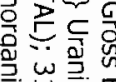

긍.

일

至完它

으를

콩ㅇㅇ

它竞畓

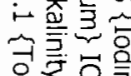

总守宫

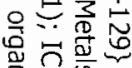

긍ㅎㅇ

学卷是只

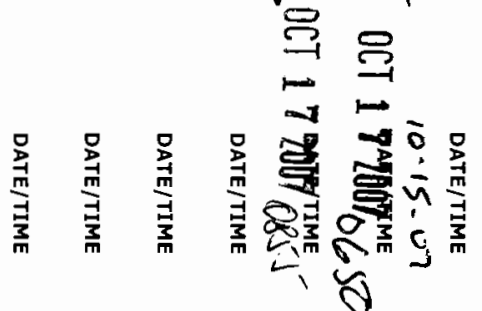
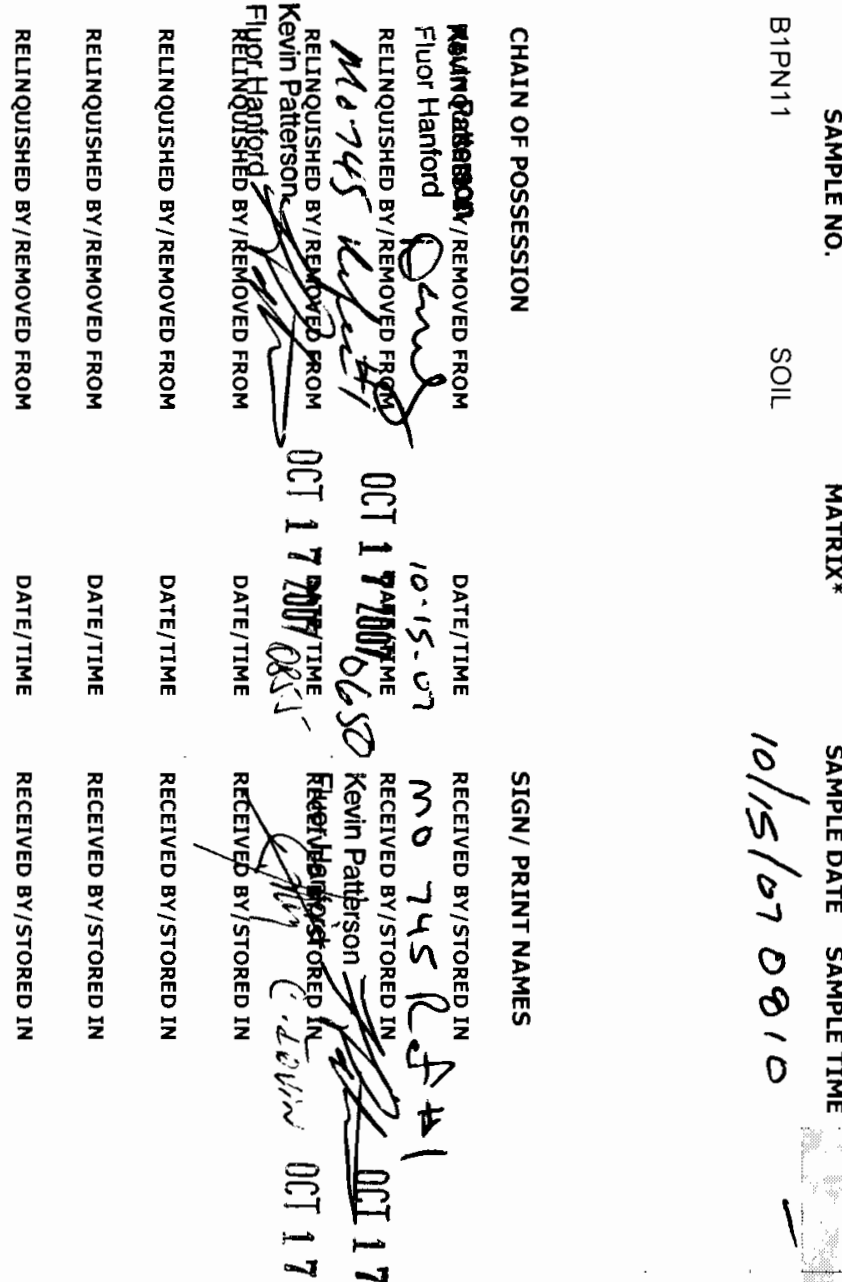

$\stackrel{\infty}{\ominus}$

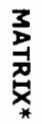

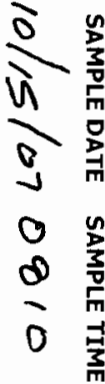

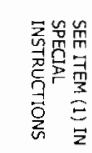

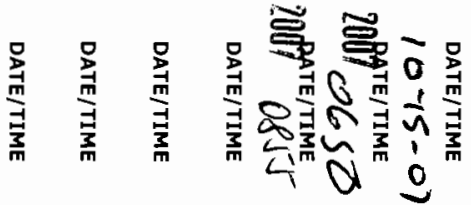

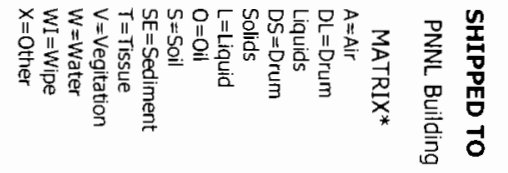

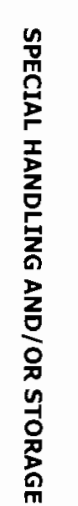

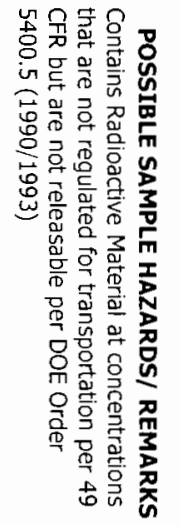

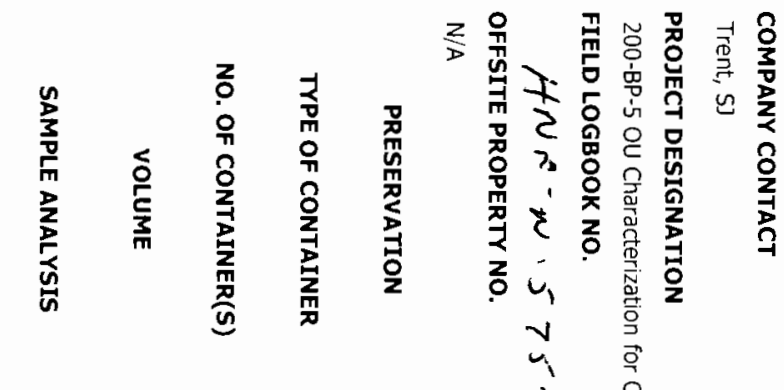

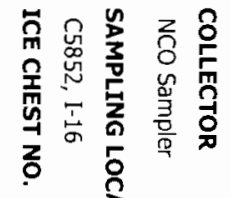

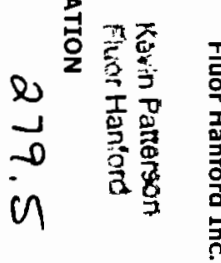

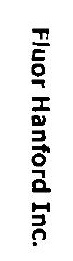

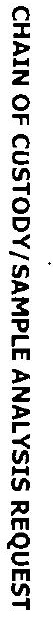

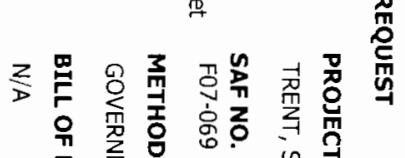

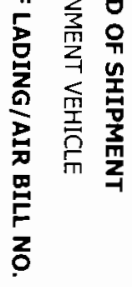

气

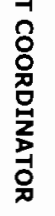

몽ㅇㅇ교귤

品 
II
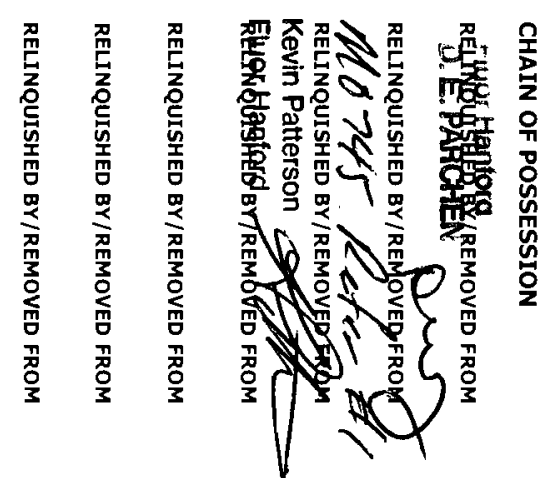

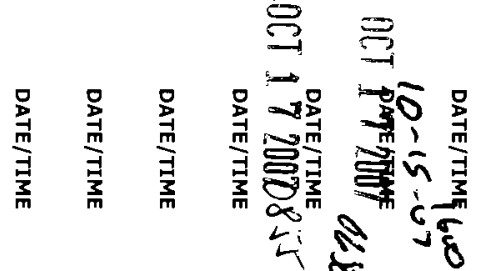

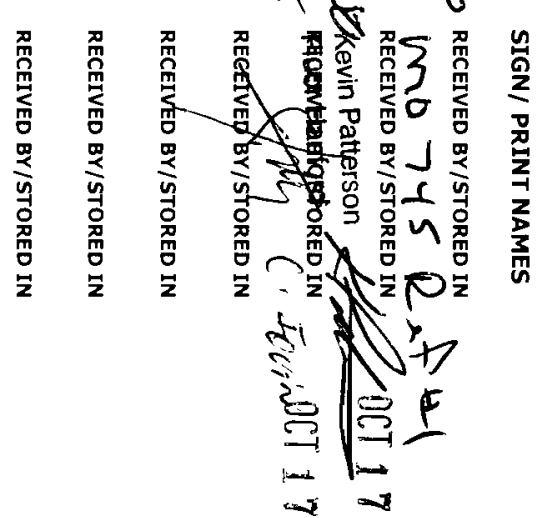

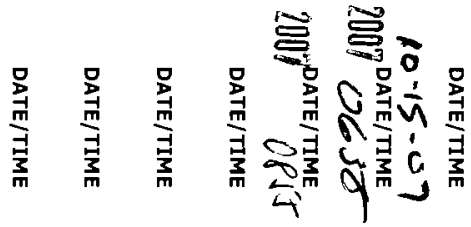

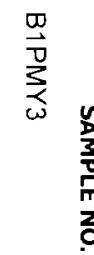

$\stackrel{\circ}{ }$

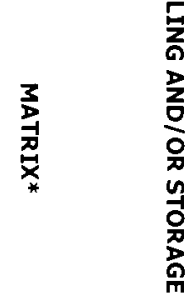

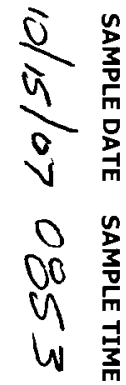

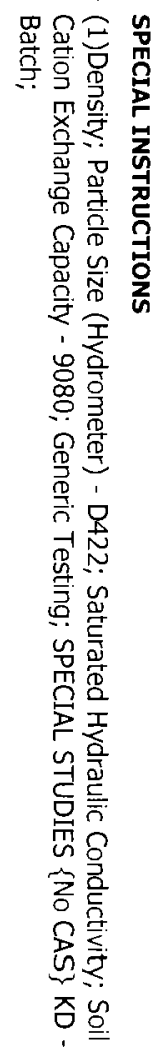

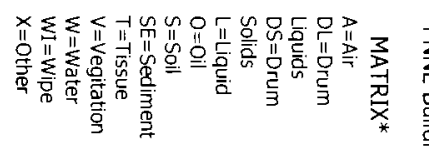

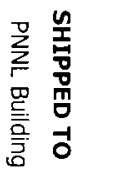

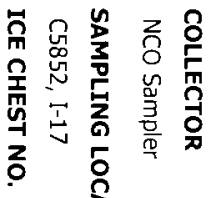
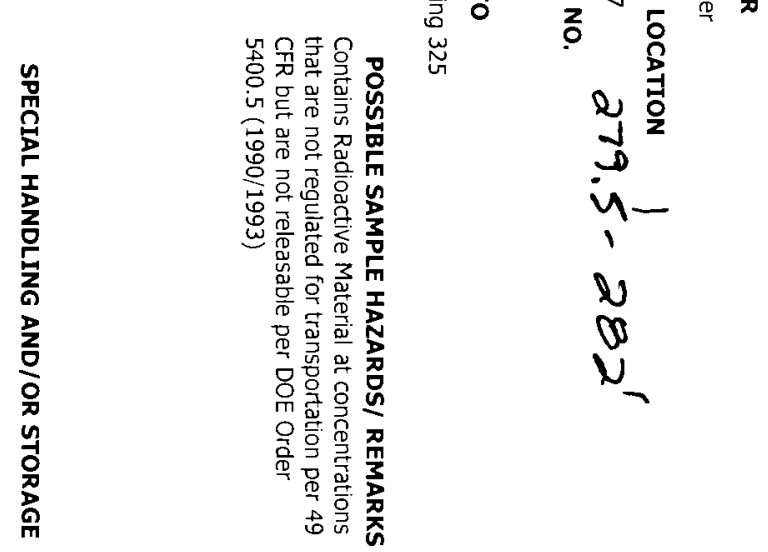

0
1
0
4
1
0
0
$n$

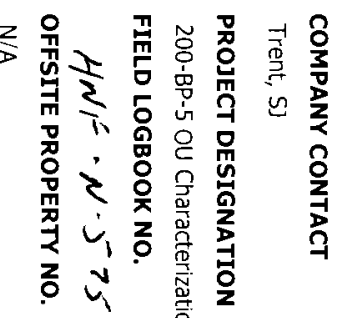

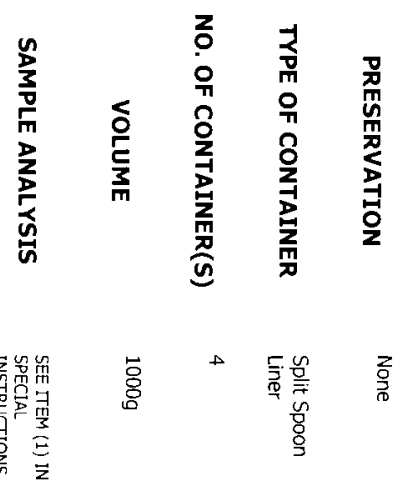

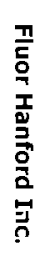
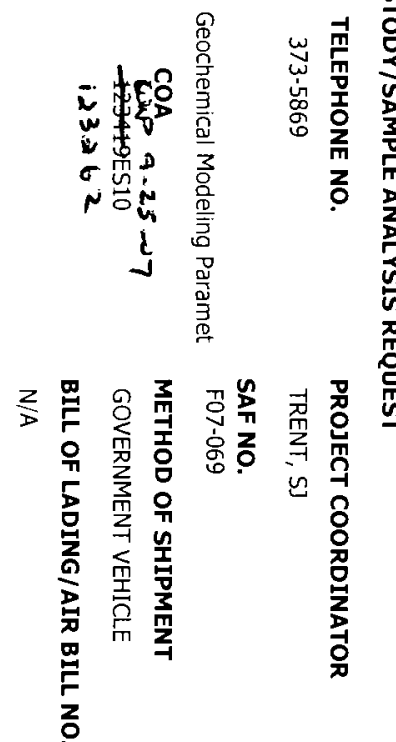

高

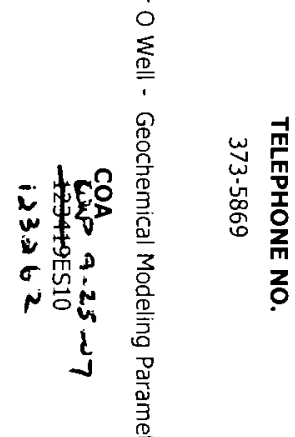

$\pi$

䎡

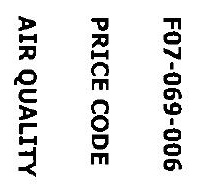

$\stackrel{\infty}{2}$

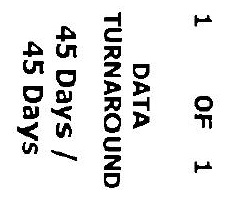



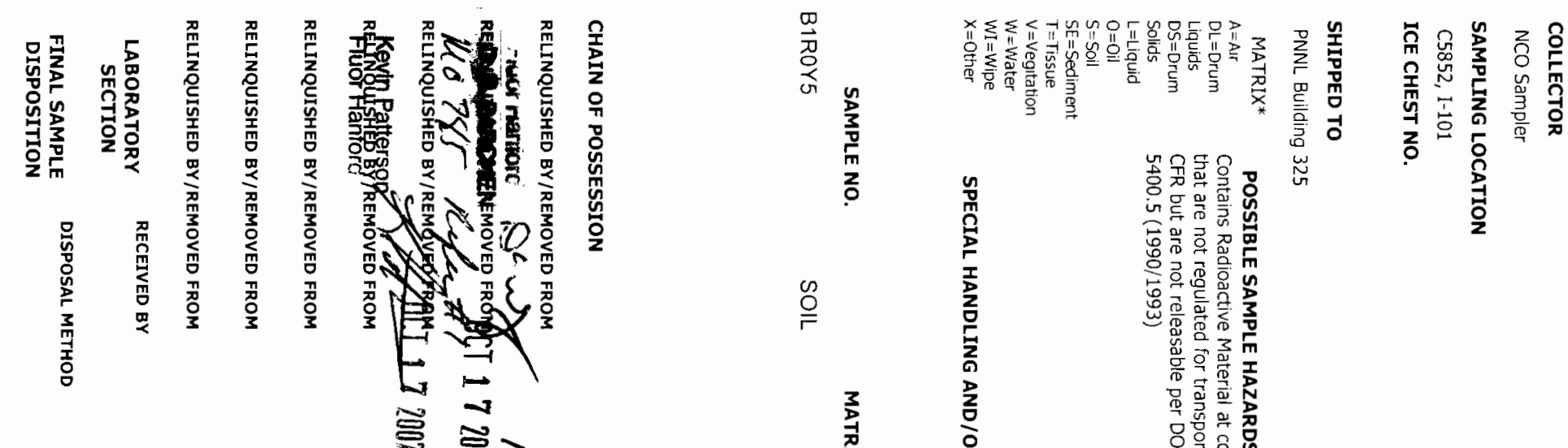

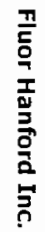

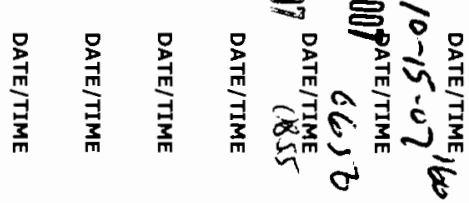

$\stackrel{\infty}{\circ}$

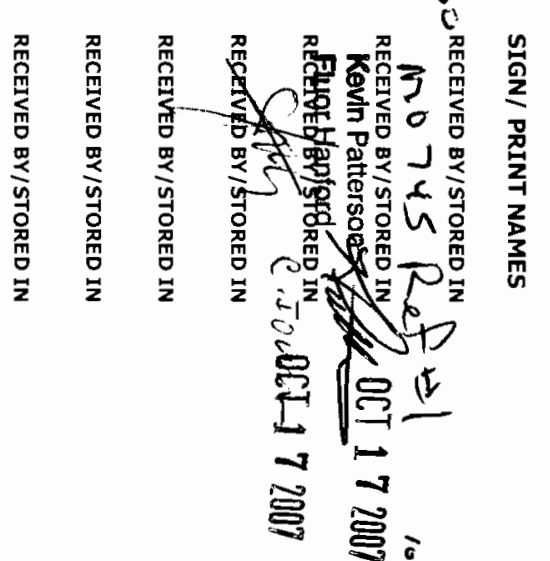

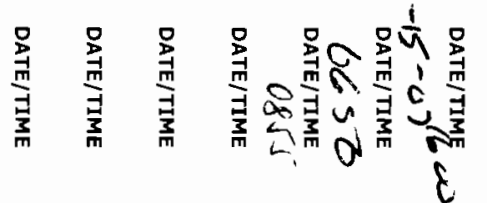

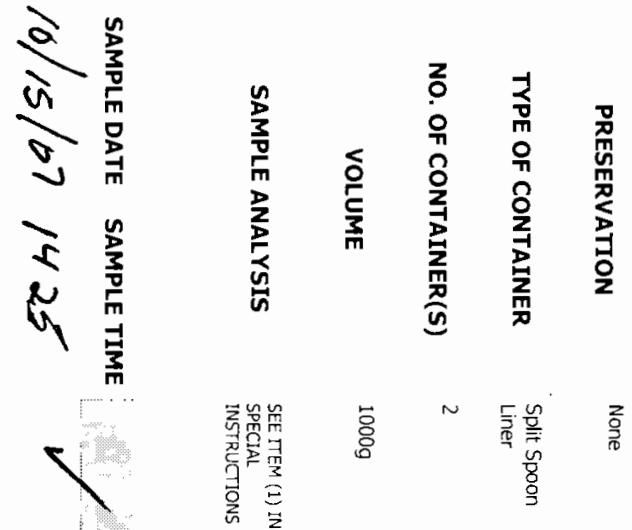

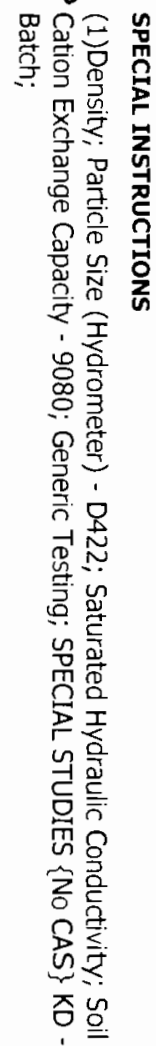

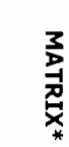

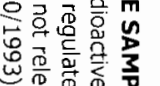

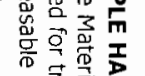

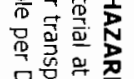

咞㔖施

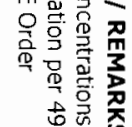

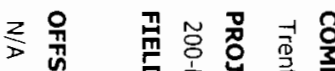

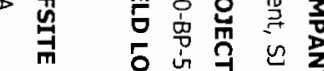

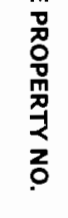

응

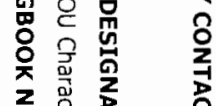

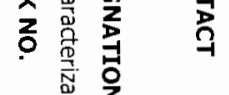

产 2

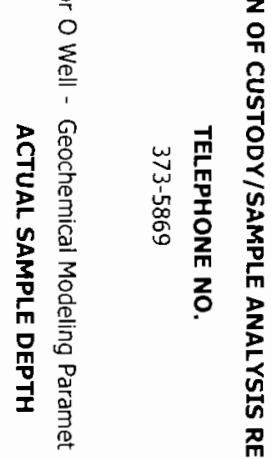

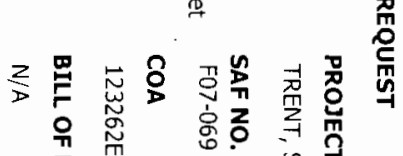

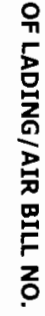

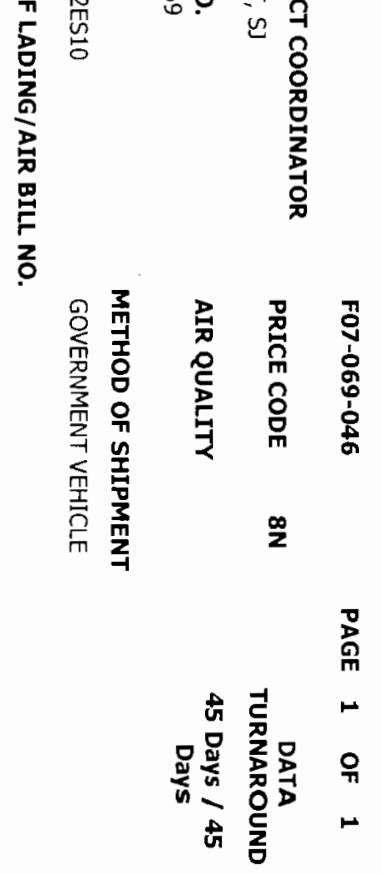



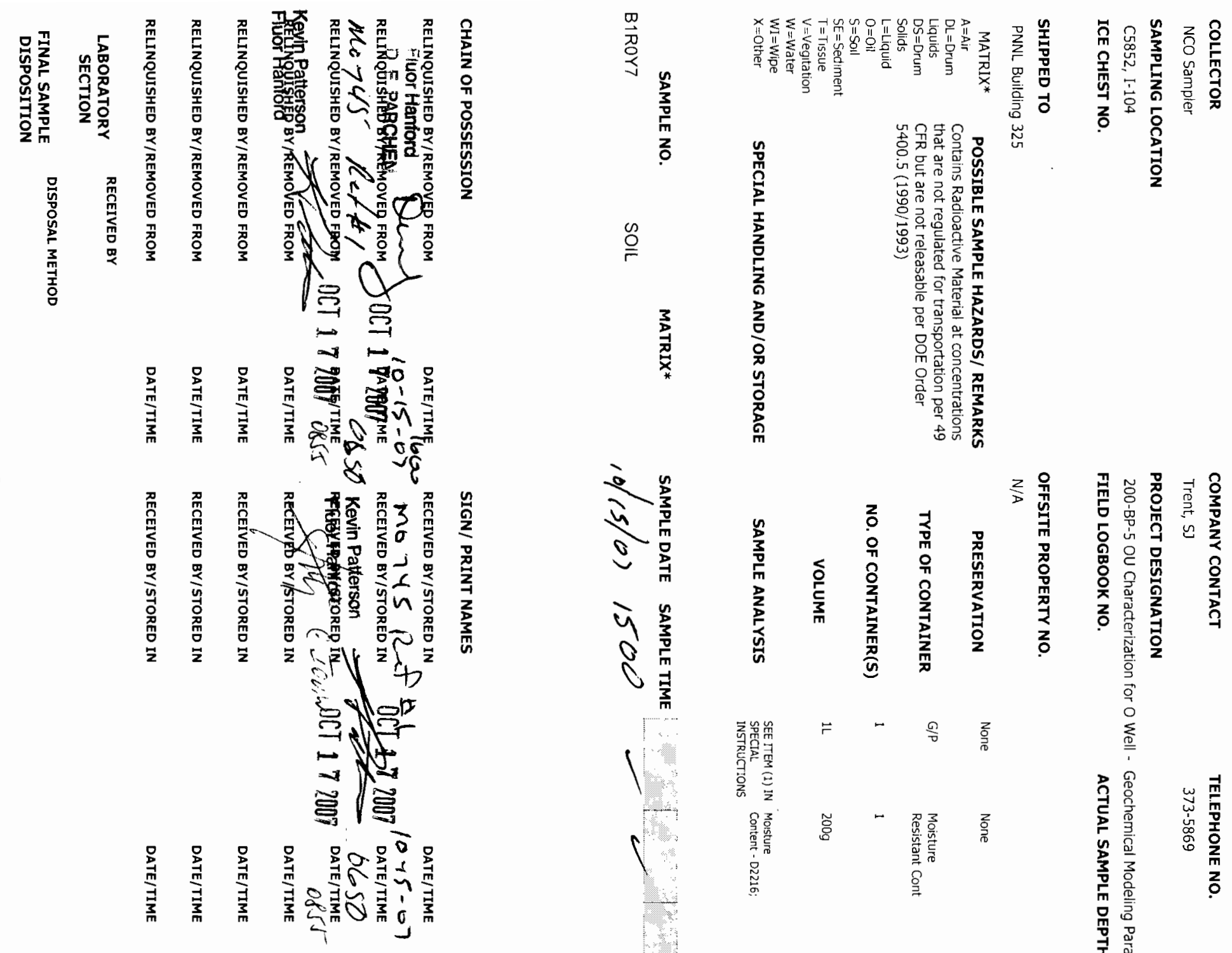

$\frac{\underline{\underline{z}}}{\underline{\underline{z}}}$

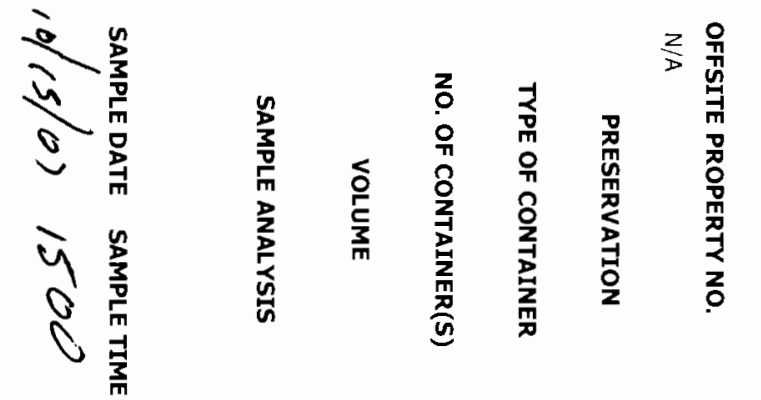

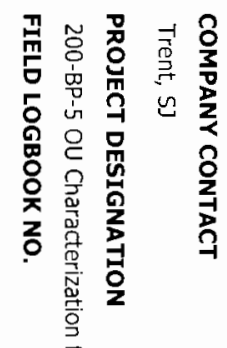

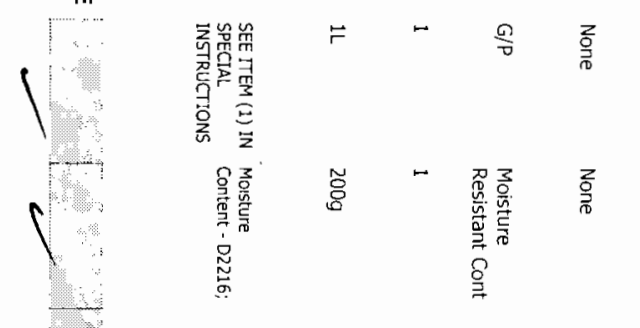

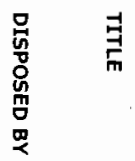
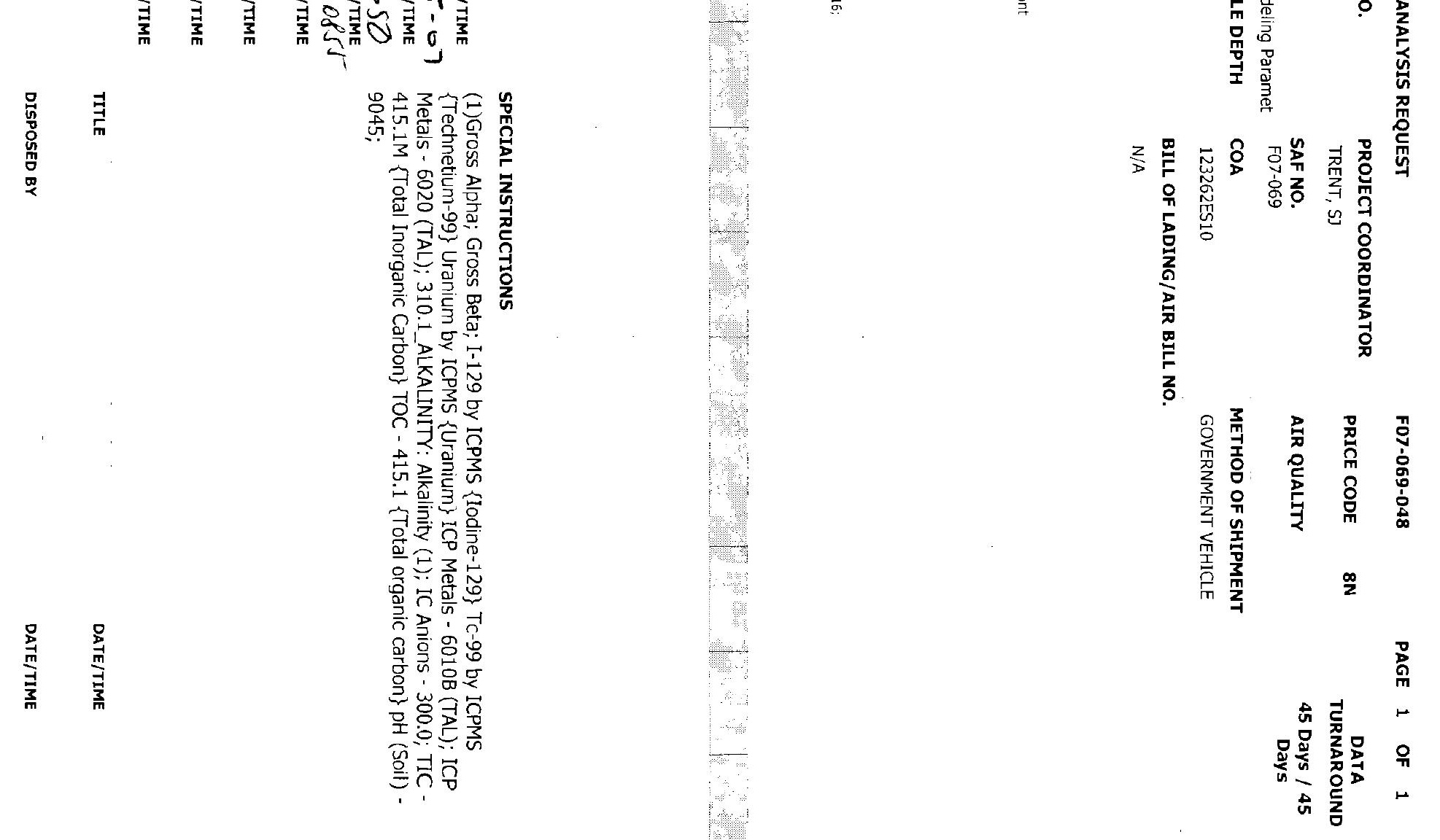


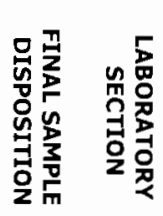

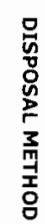

$\$$

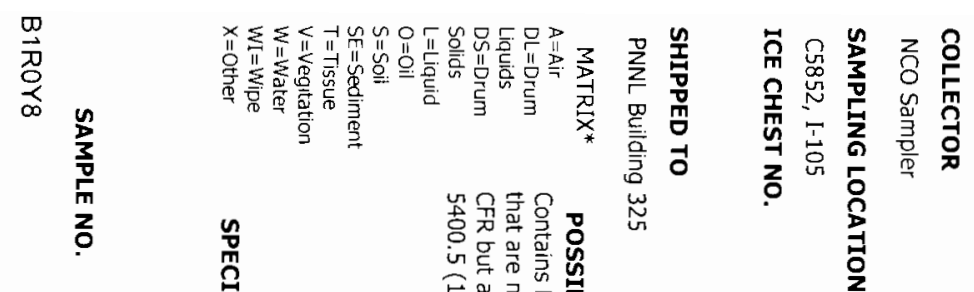

$\stackrel{\leftrightarrow}{\circ}$

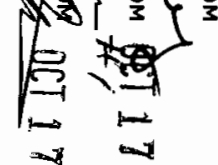

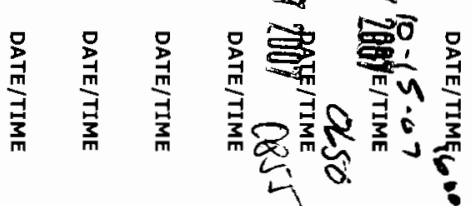

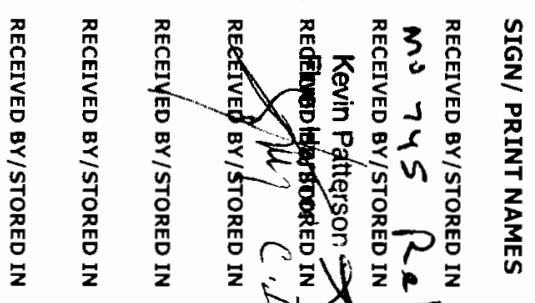

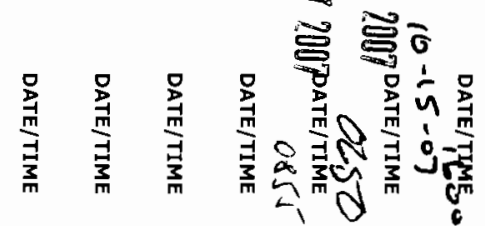

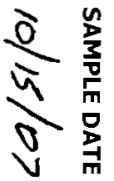

$\hat{4} \frac{\sqrt{\frac{1}{3}}}{\overline{0}}$
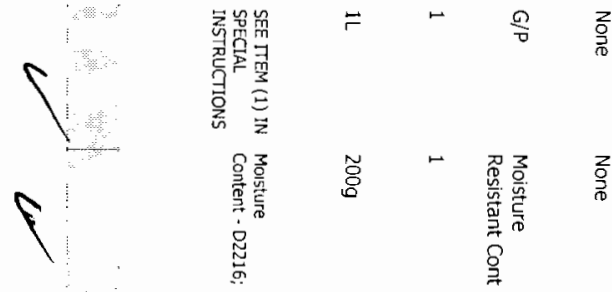

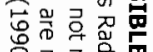

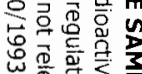

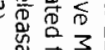

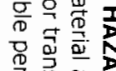

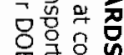

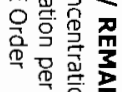

古急赑

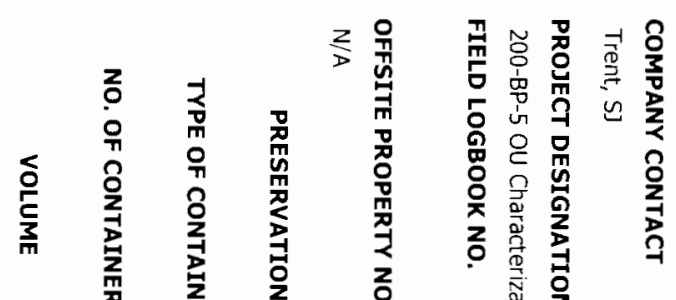

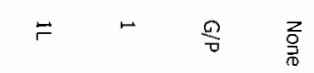

8
0
0
0

$\exists$
$\mathbf{n}$

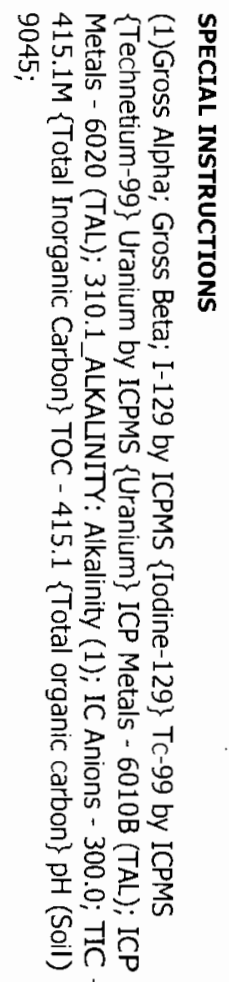

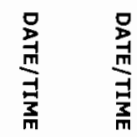

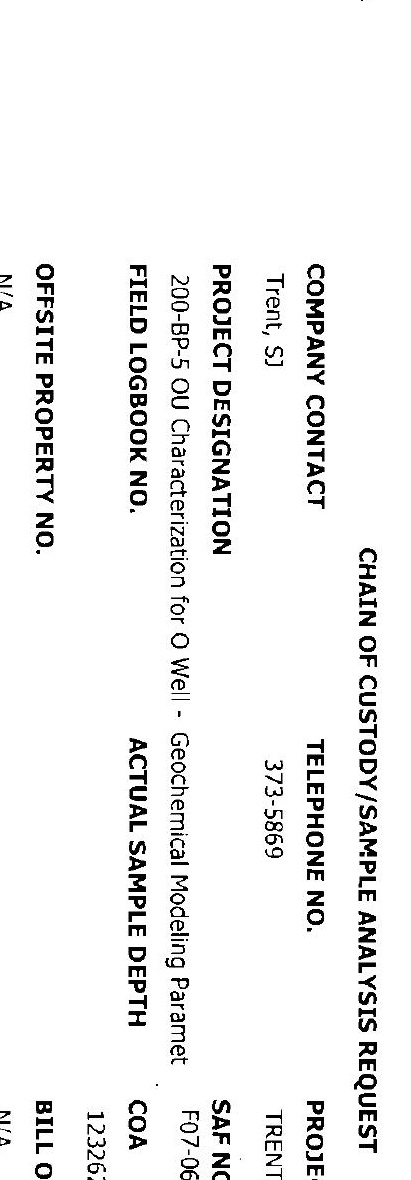

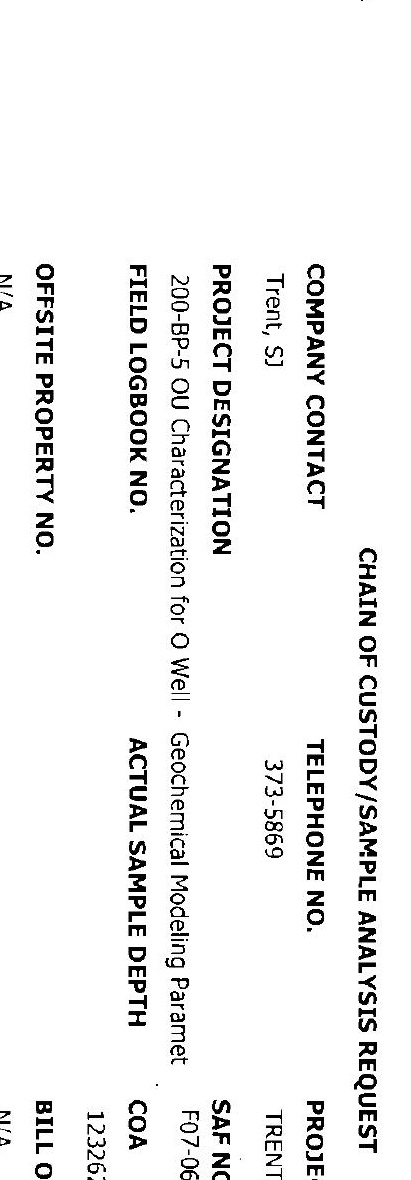

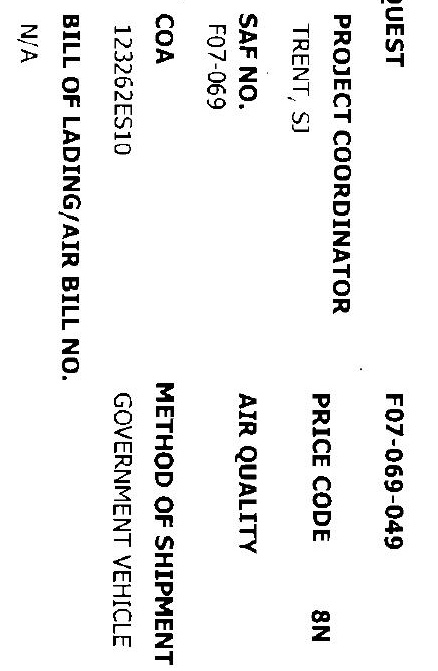



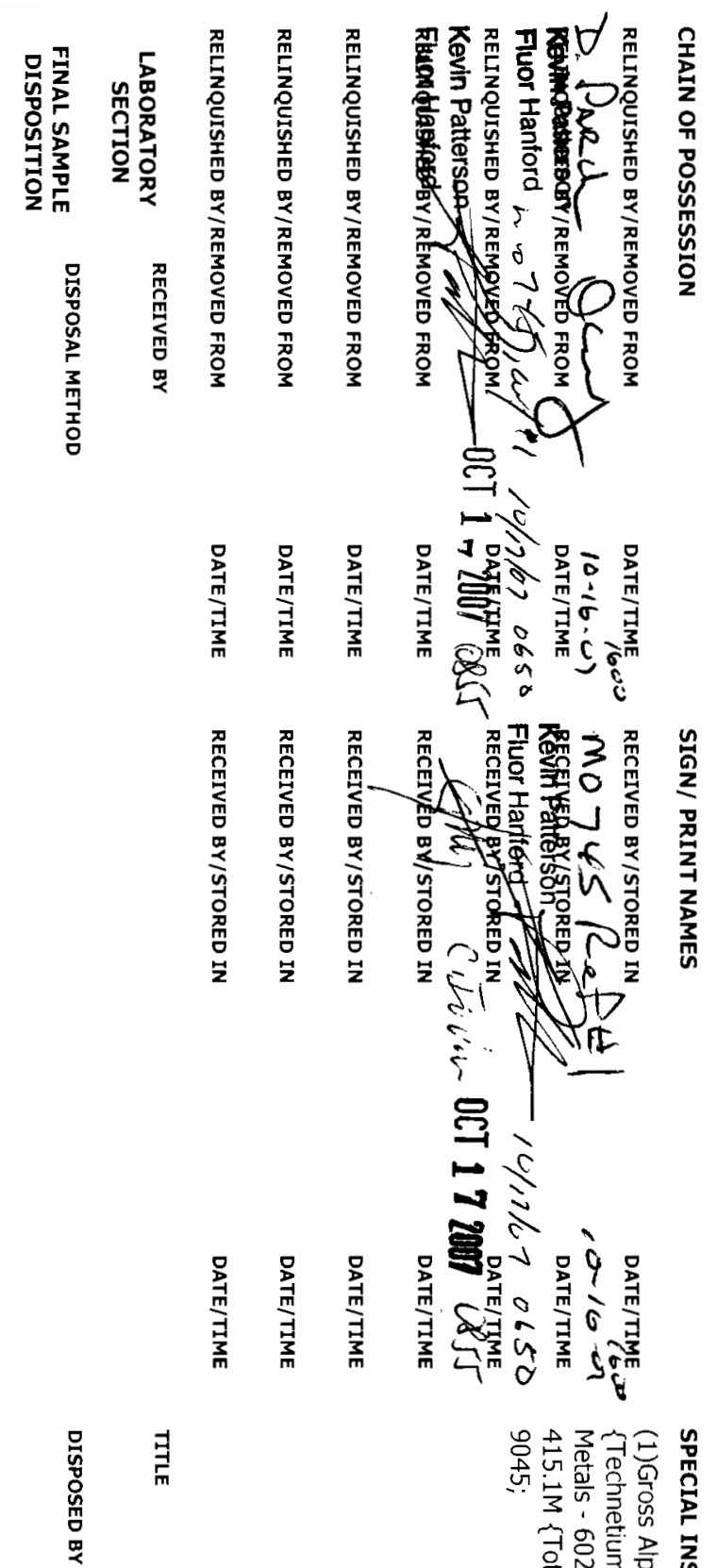

I

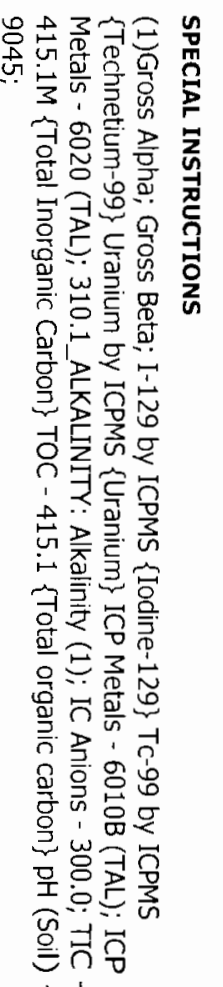

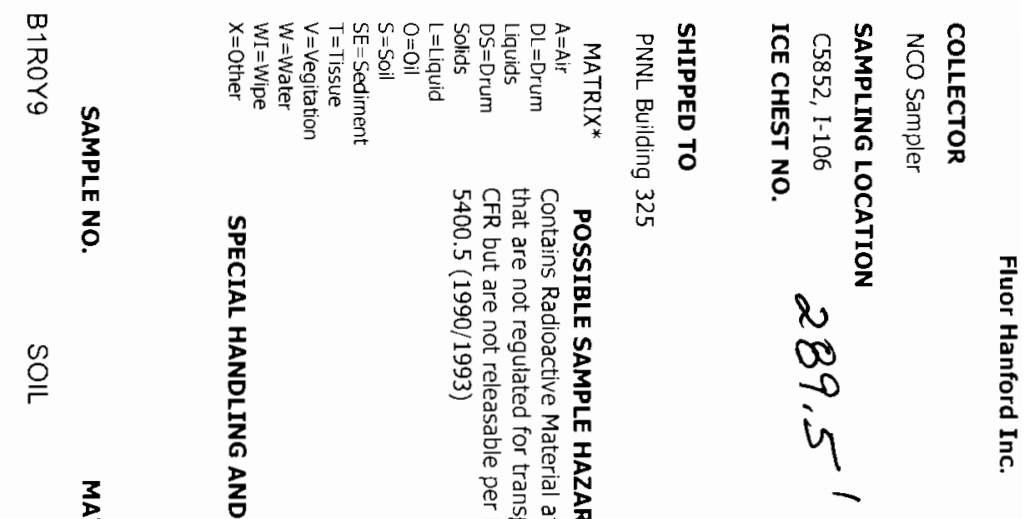

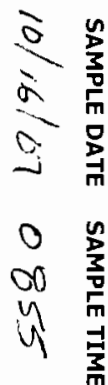
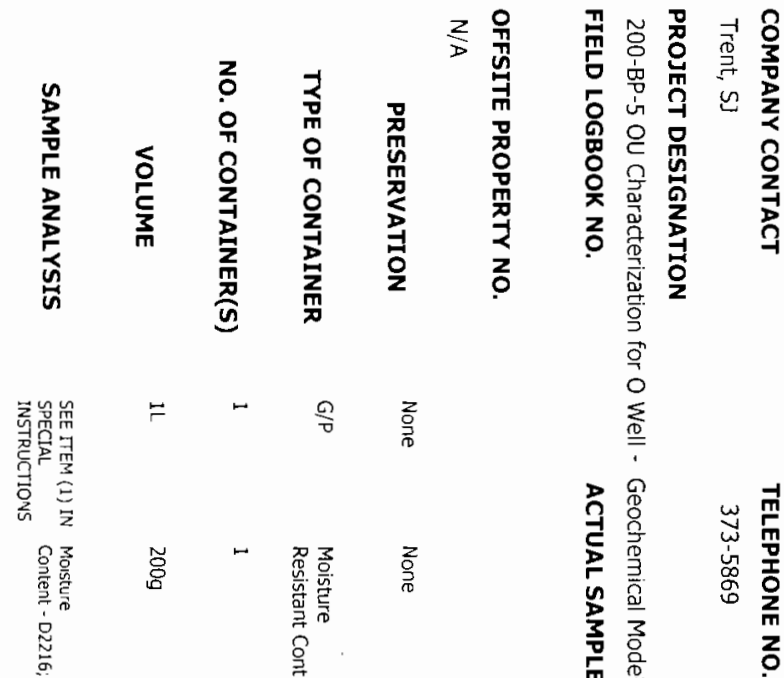

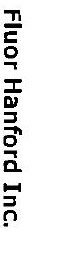




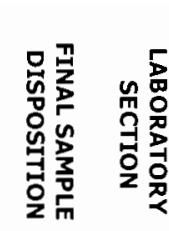

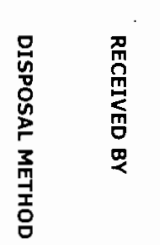

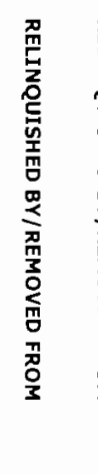

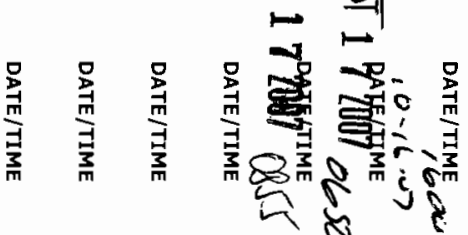

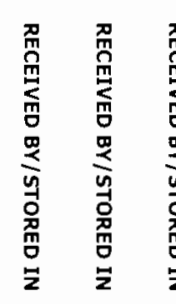

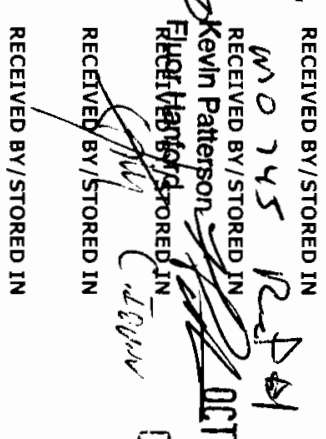

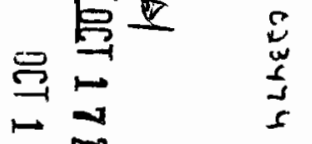

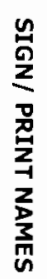

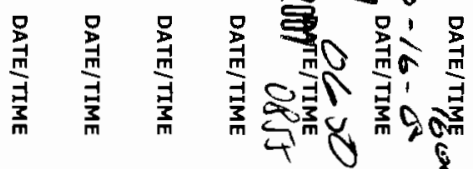

量

$\underset{m}{\overrightarrow{3}}$

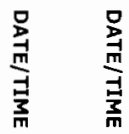

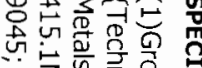

了。家员

궁루를

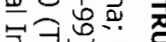

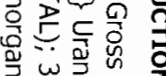

言岂总罾

它范

空宪

응츤

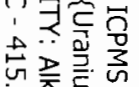

语总

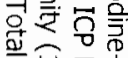

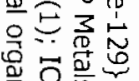

극군

욱용

항

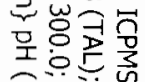

品䓃守
$0 \rightarrow 3 \mathrm{C}$

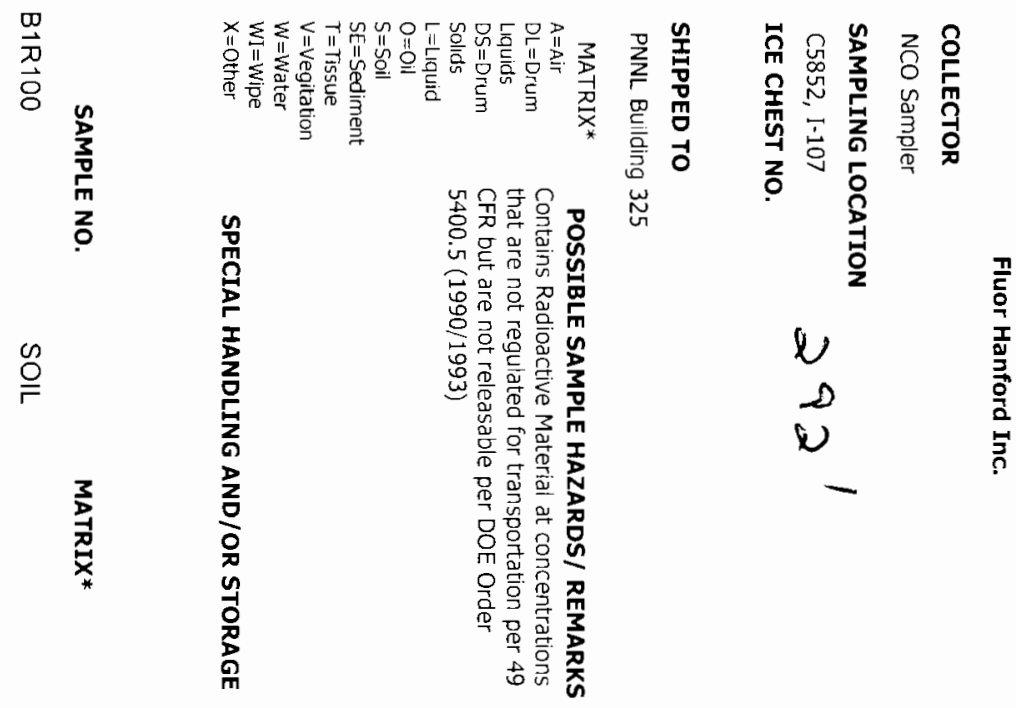

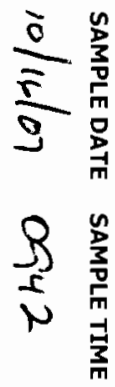

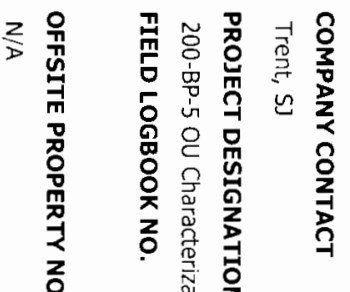

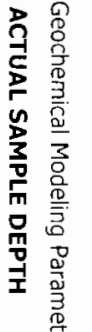

剀

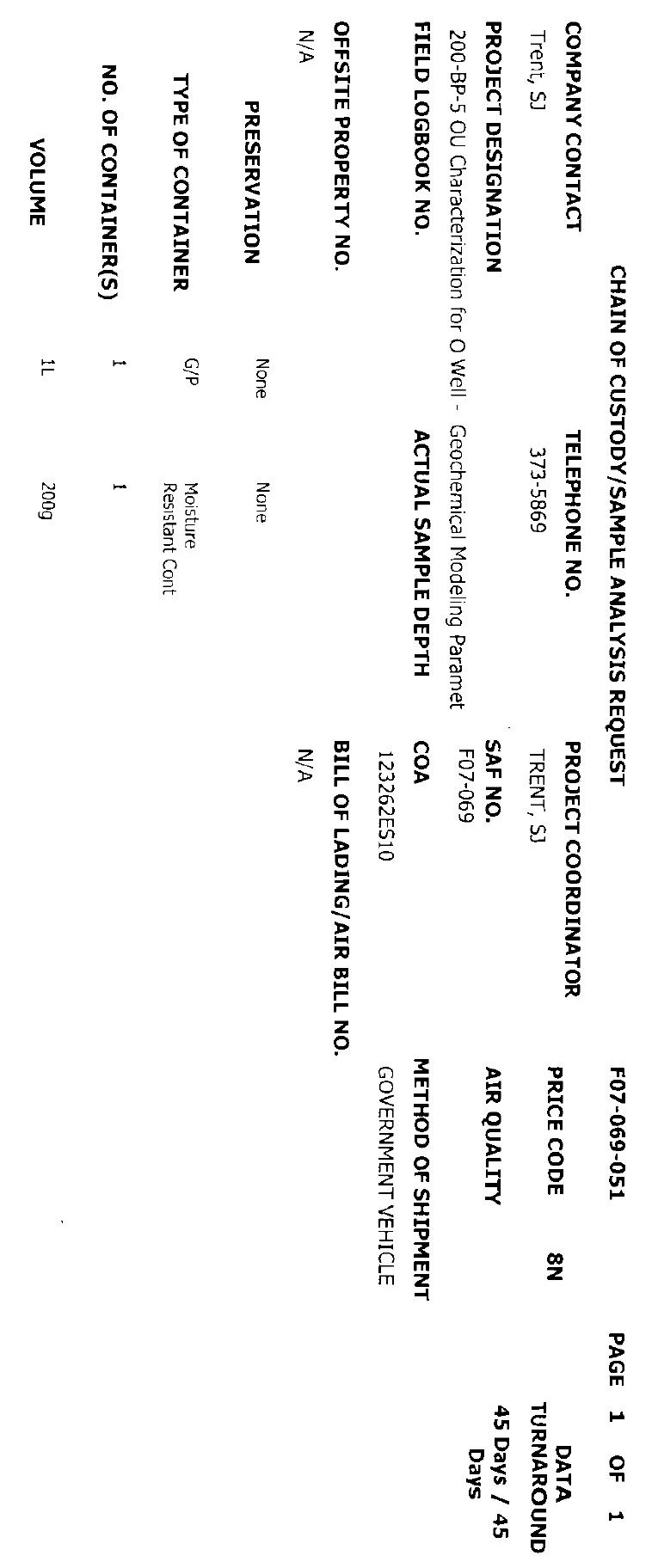




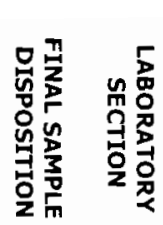

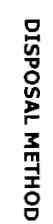

1

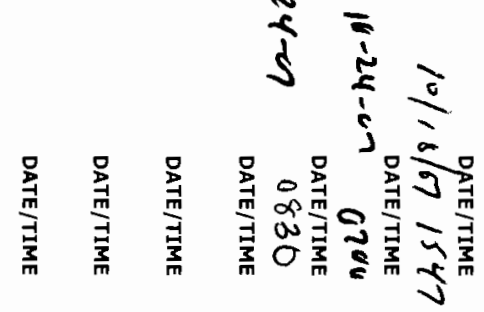

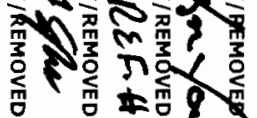

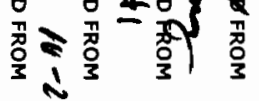

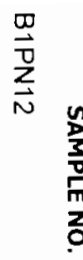

$\stackrel{0}{1}$

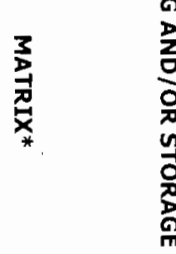

$\frac{3}{2}$

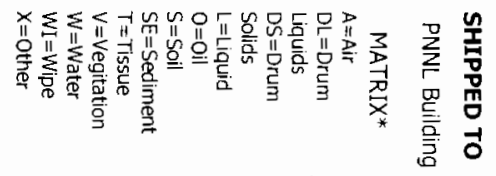

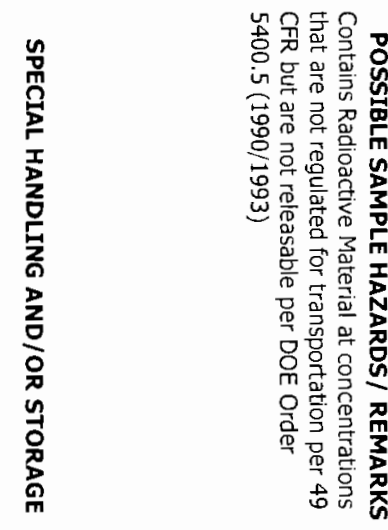

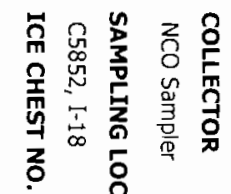

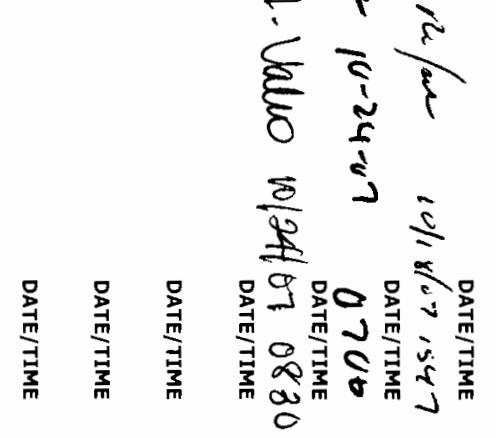

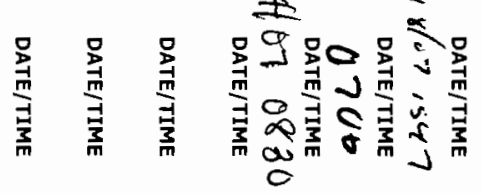

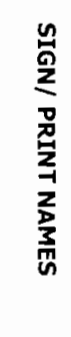

$\frac{\overline{0}}{\frac{0}{0}}$

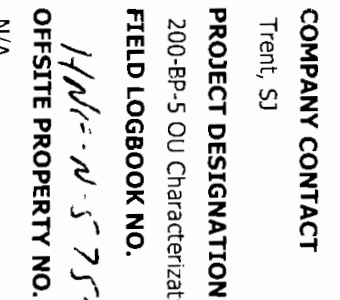

굴
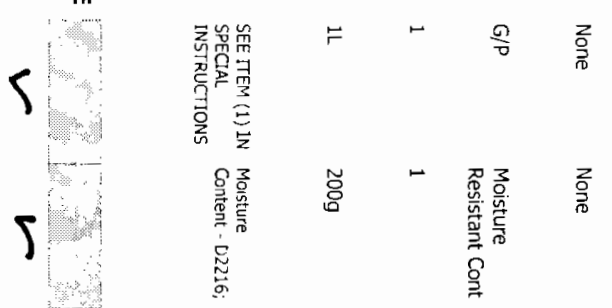

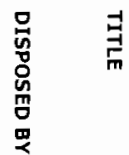

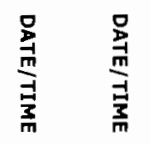
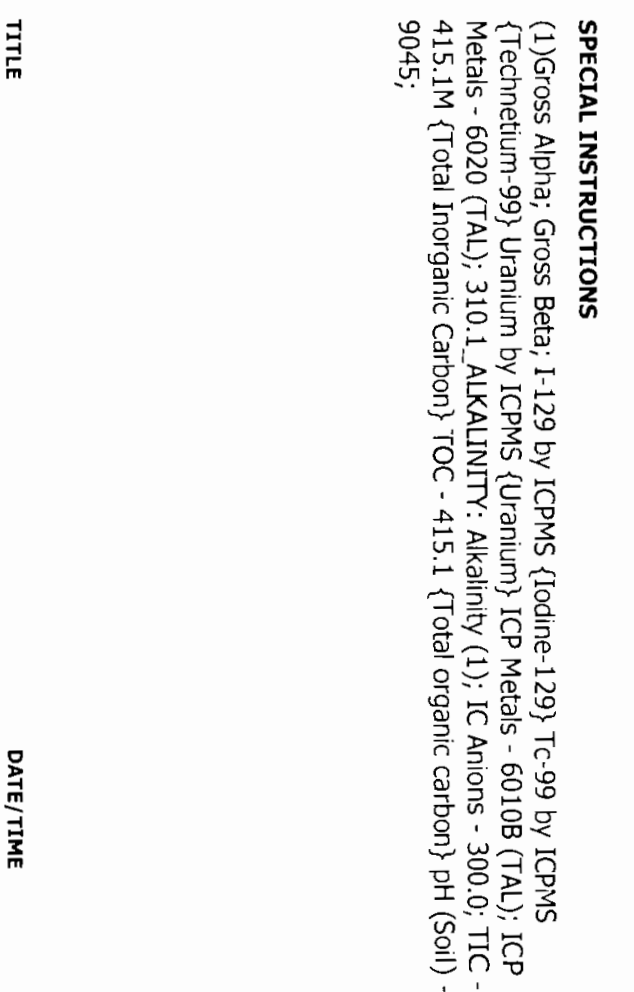
(1) 


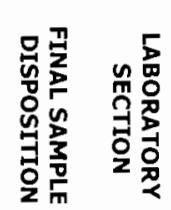
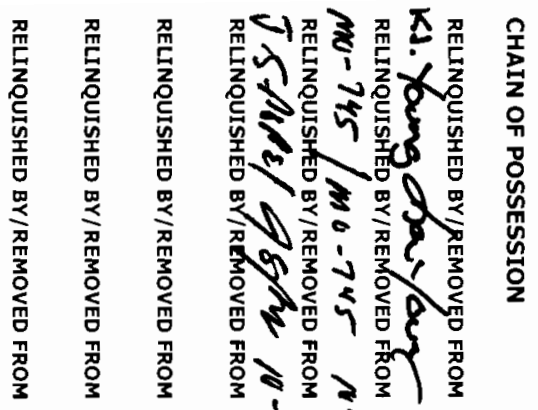

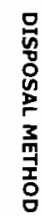

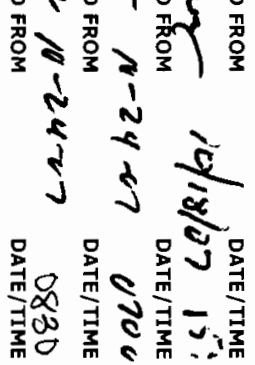

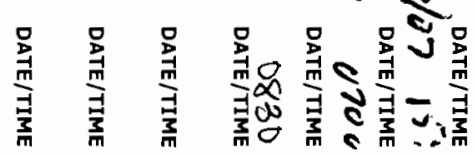
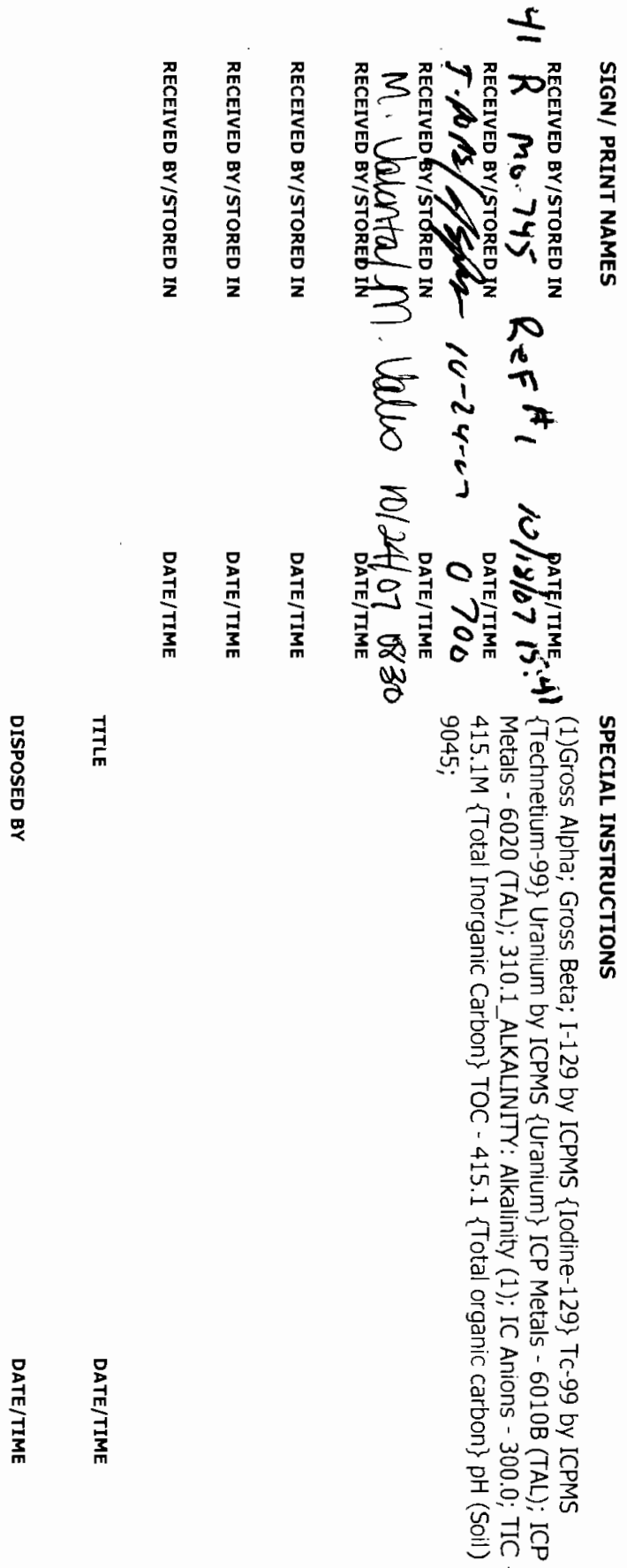

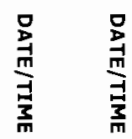

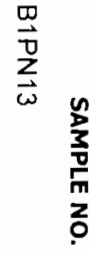

$\stackrel{\leftrightarrow}{\circ}$

$\frac{\overline{0}}{9}$

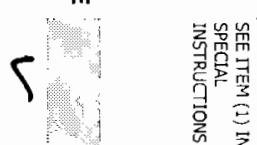

S

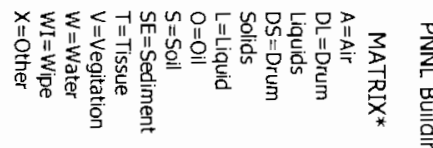

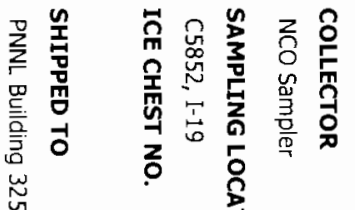

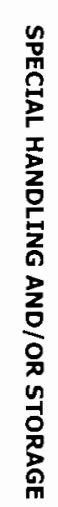

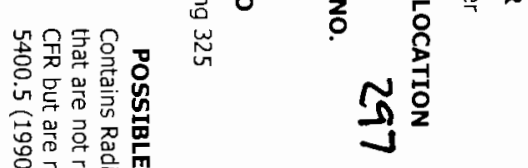

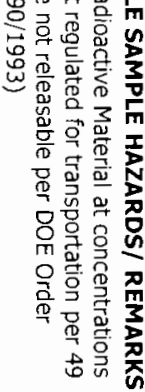

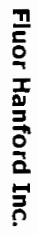

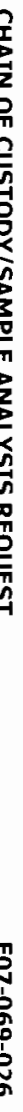

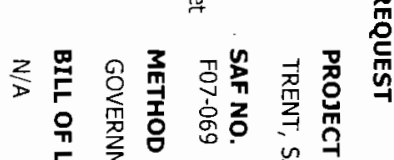

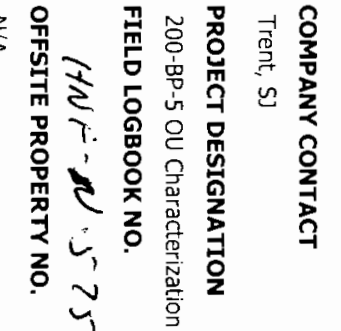

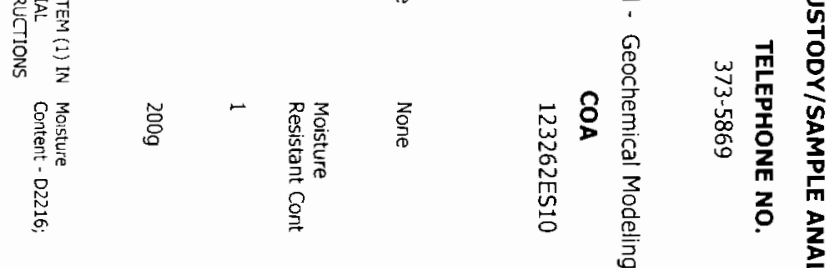

要

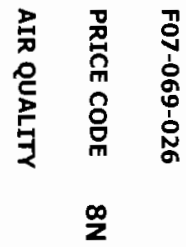

䓪

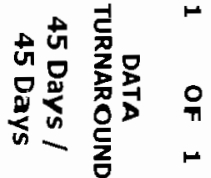




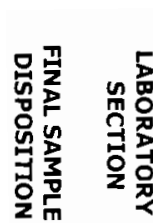

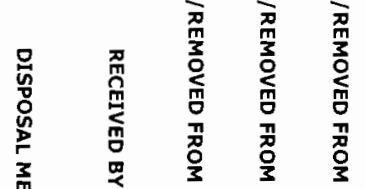

魚

吾

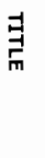

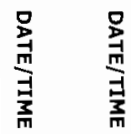

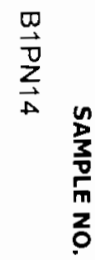

$\stackrel{\oiiint}{\rightleftharpoons}$

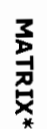

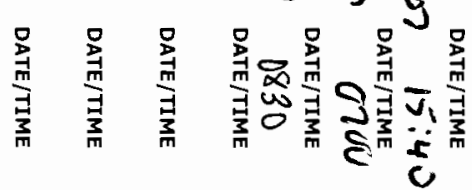

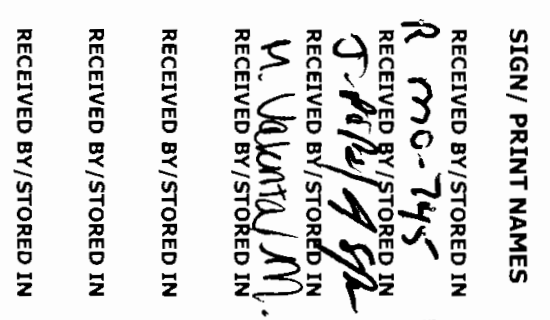

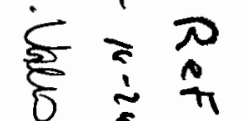

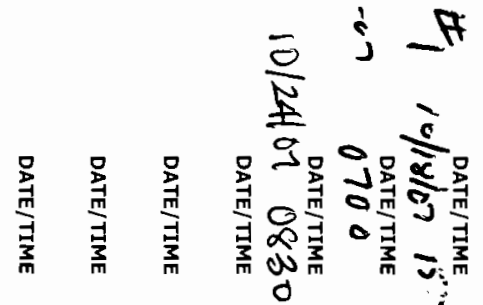

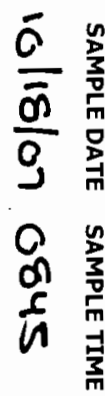

「人

S

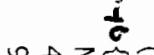

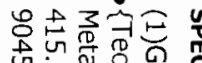

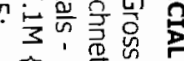

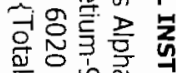

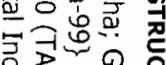

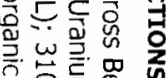

它票塄

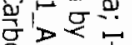

究官灾

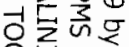

길ㄷำ

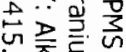

$\because$ 豞

구웡응

옹

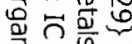

고

응ㅇㅇㅇ

홀 它.

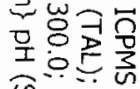

范完

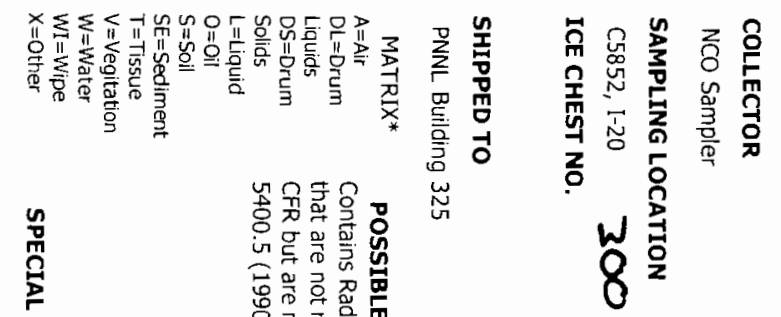

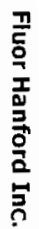

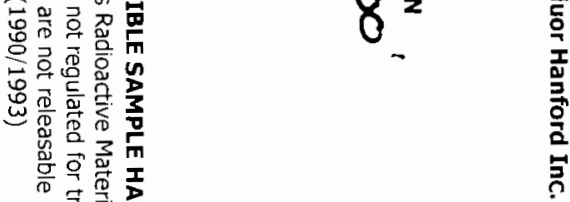

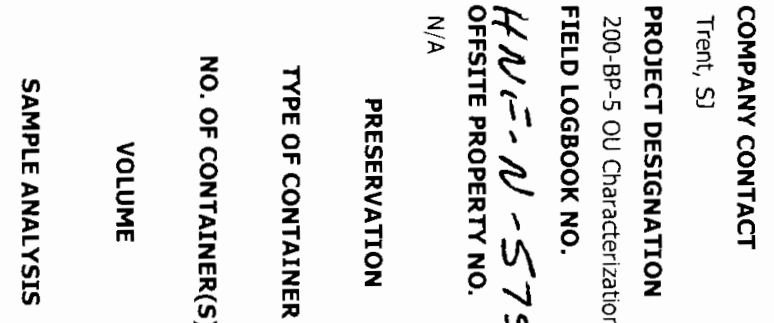

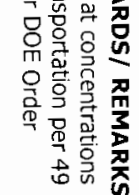

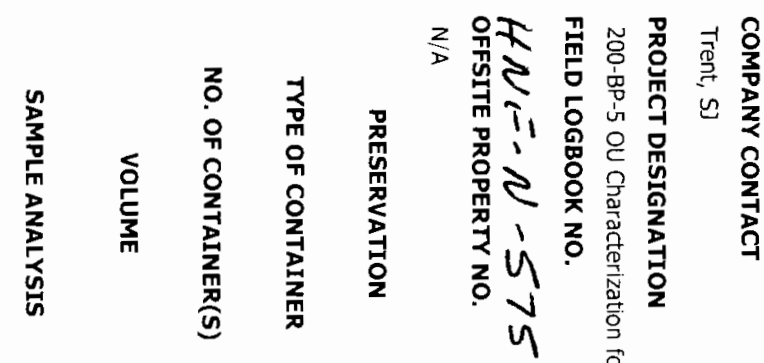

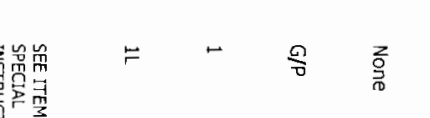

u

竞

范 


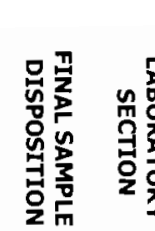

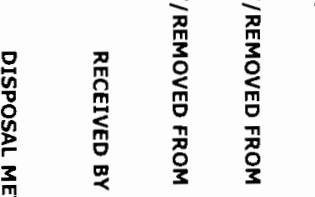



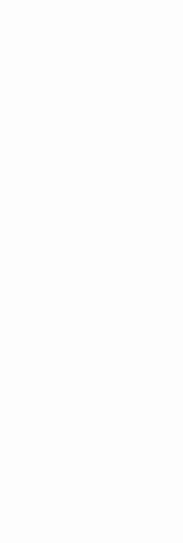

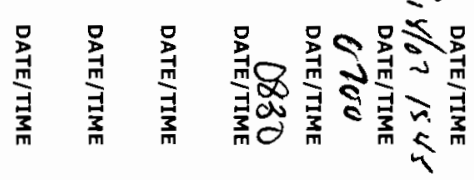

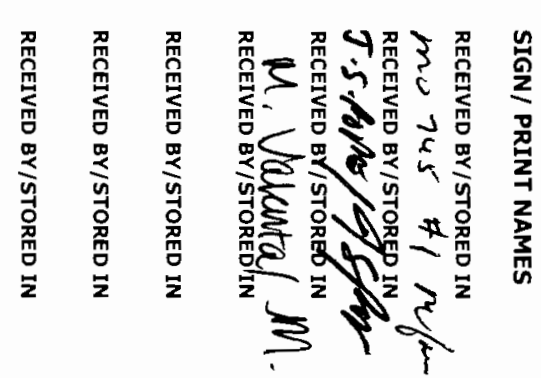

है

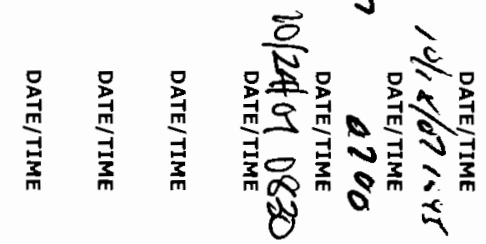

$\vec{\exists}$

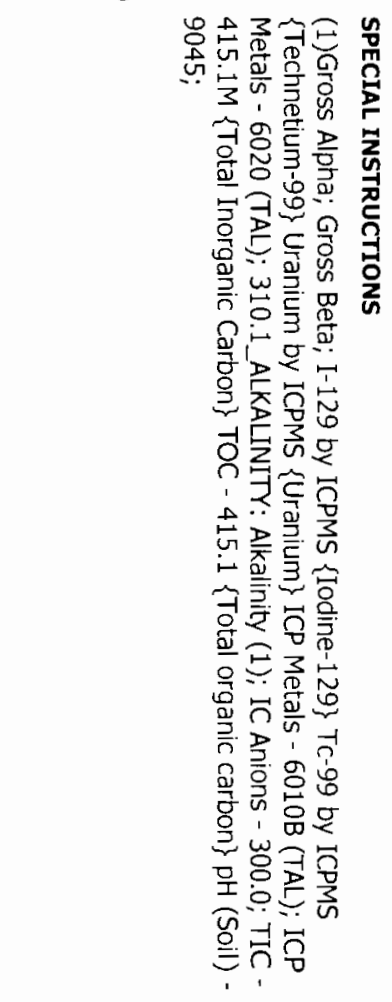

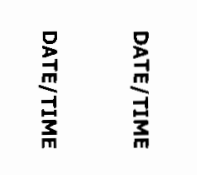

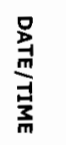

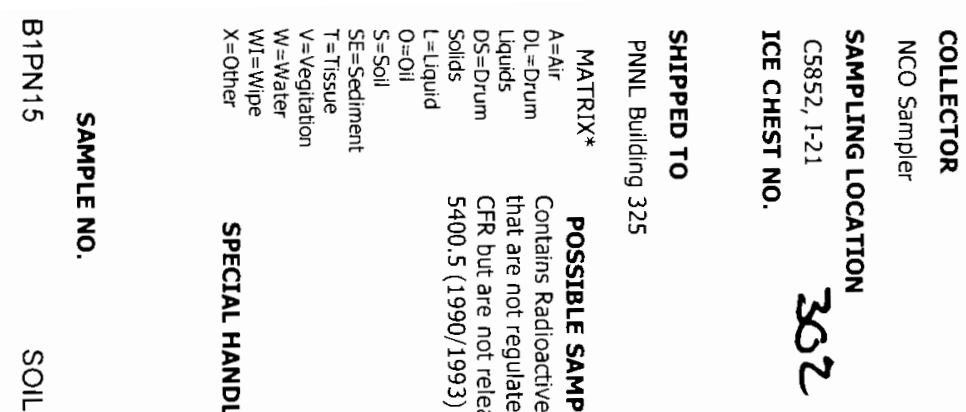

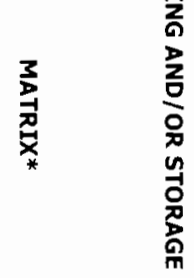

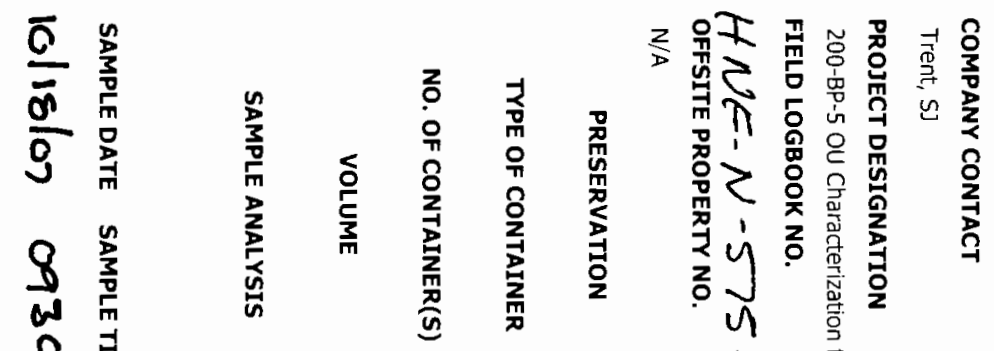
$\mathfrak{N}^{\prime} \quad \stackrel{\substack{0 \\ \sum_{0}^{0}}}{\underline{0}}$

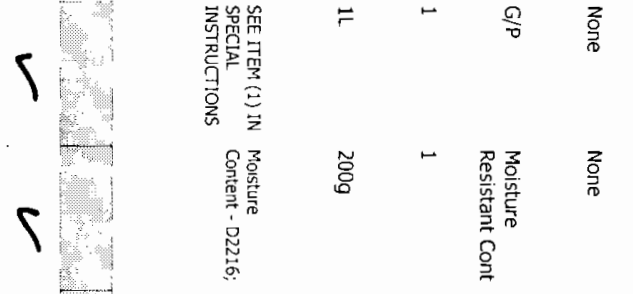




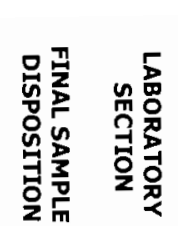

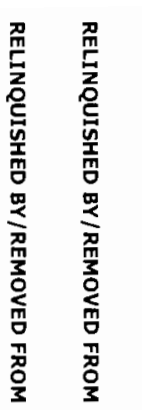

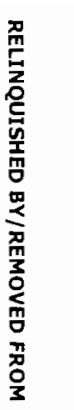

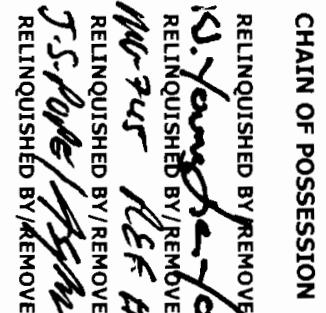

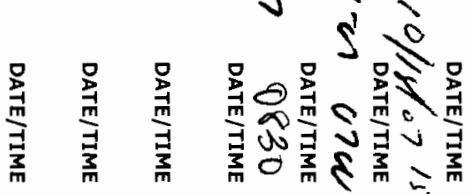

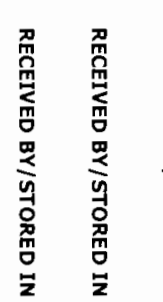

के
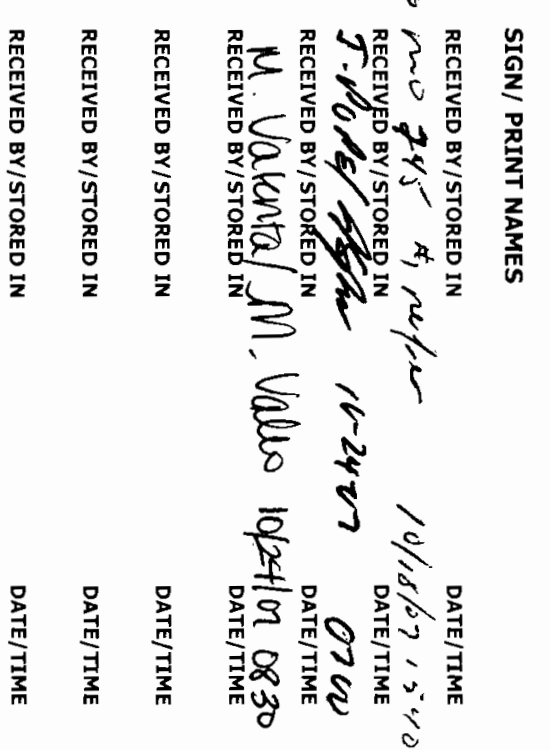

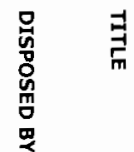

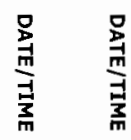

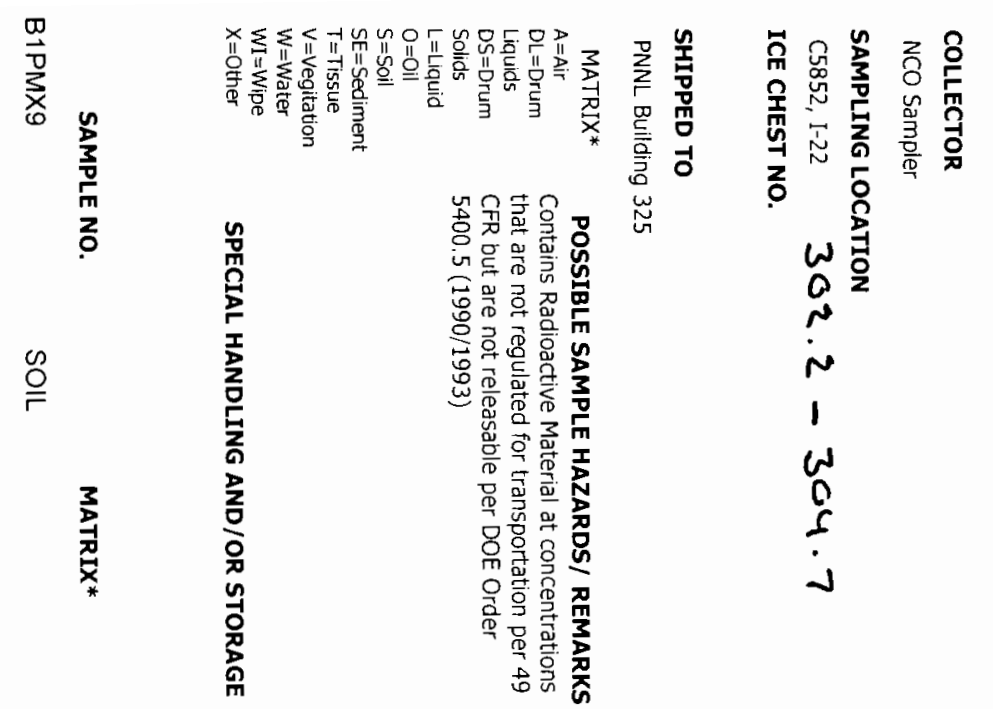

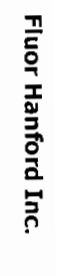

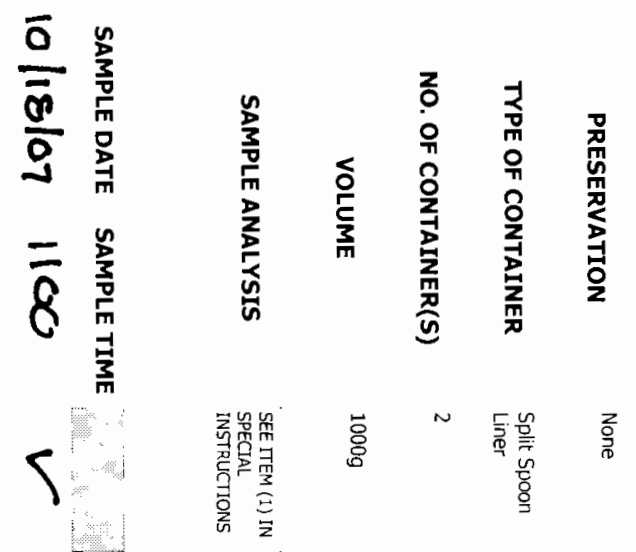

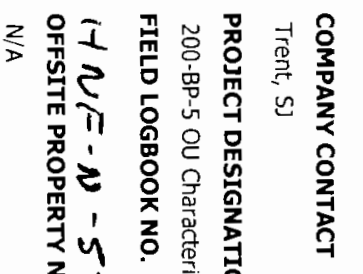

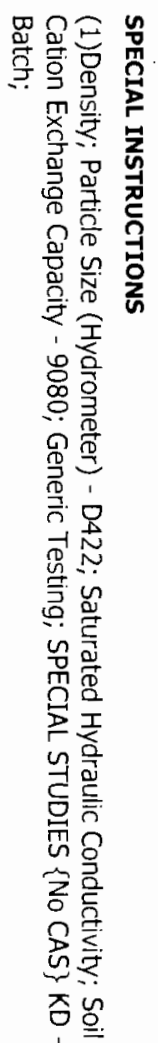

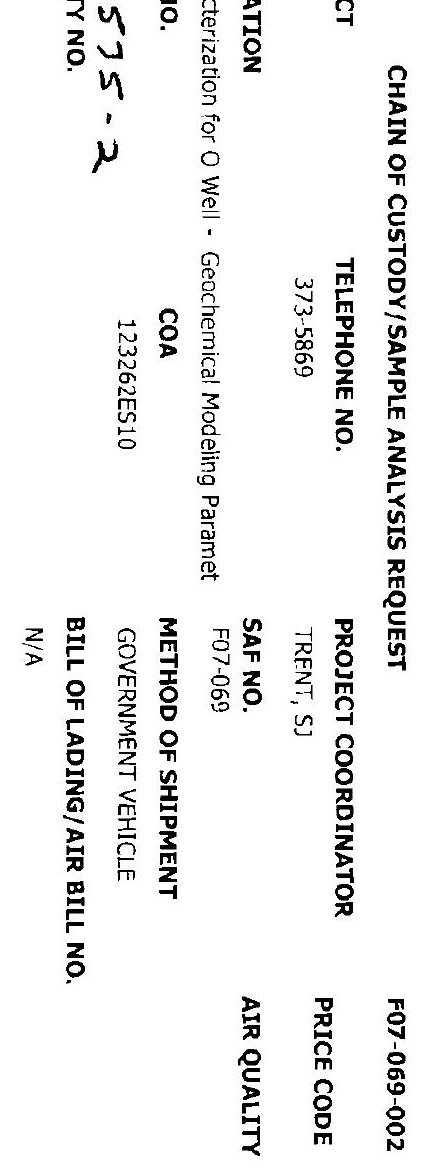

$\frac{\infty}{2}$ 
镐
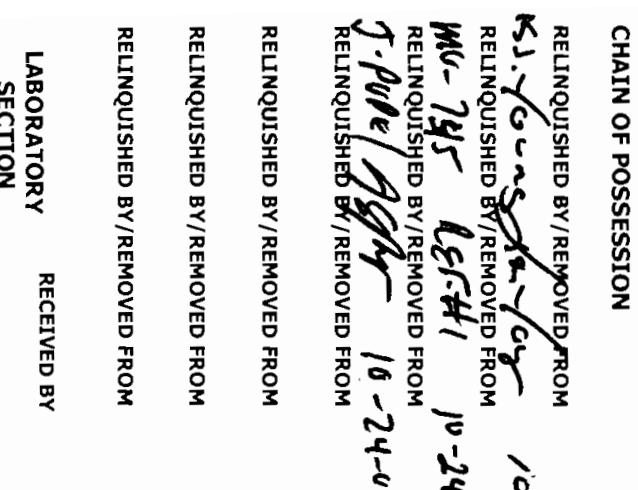

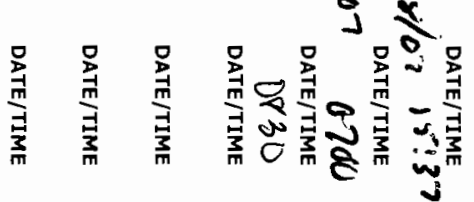

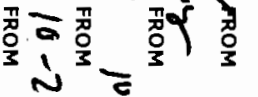

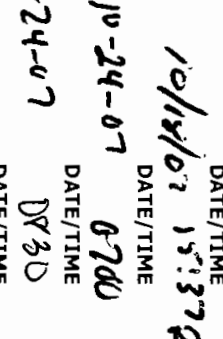
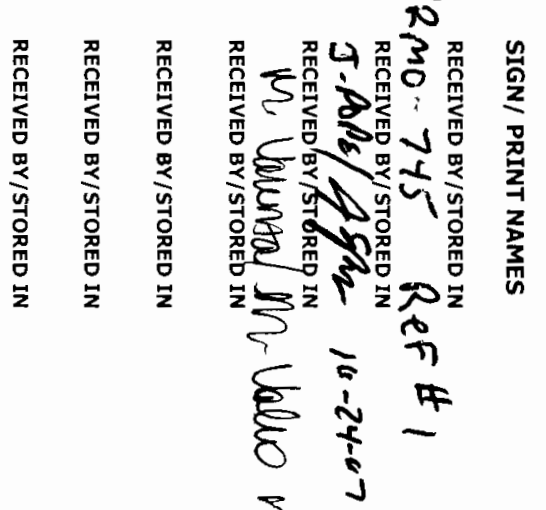

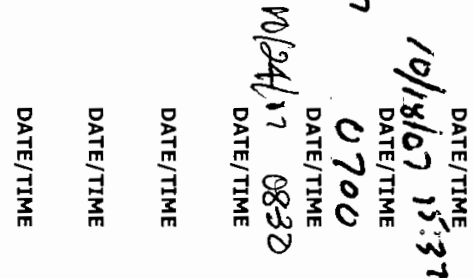
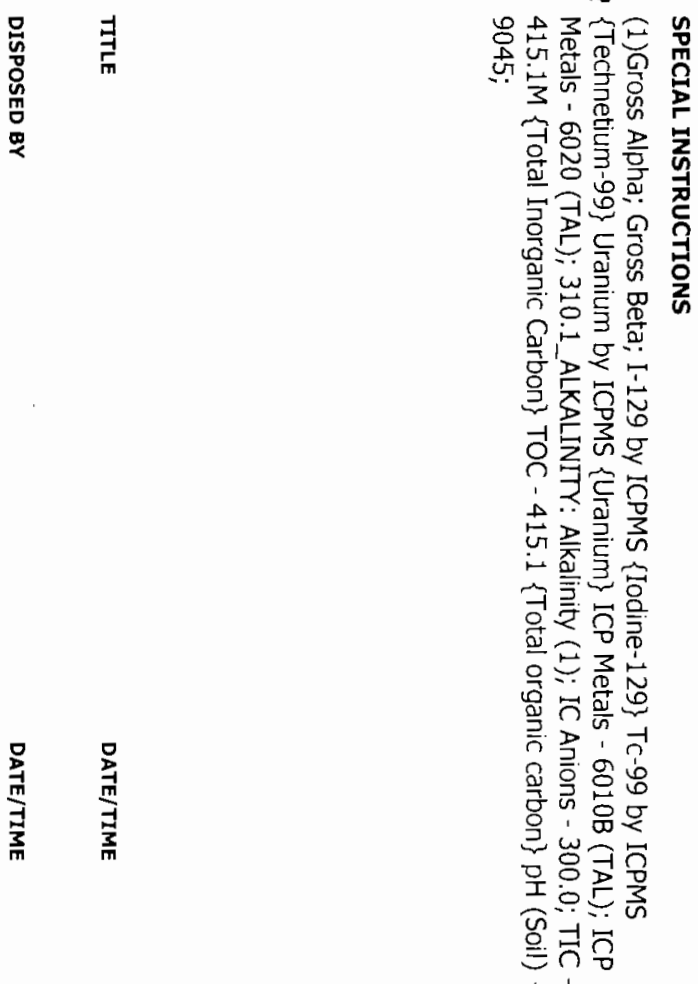

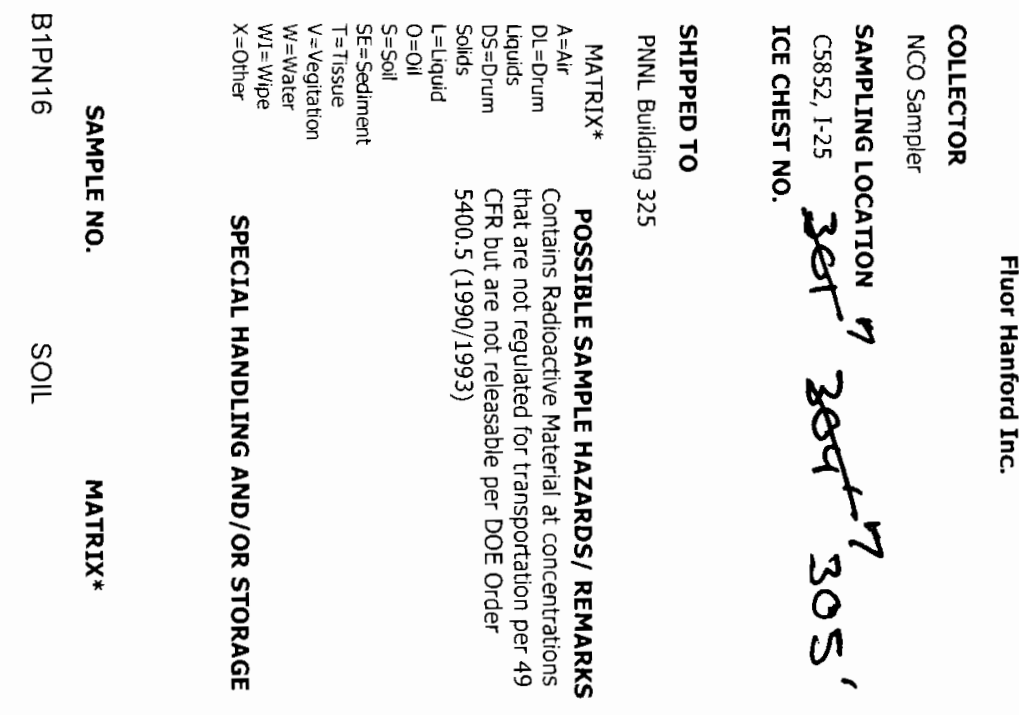

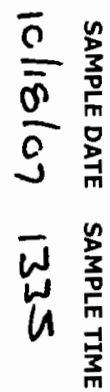

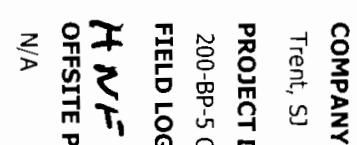

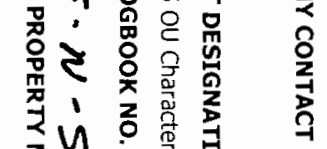

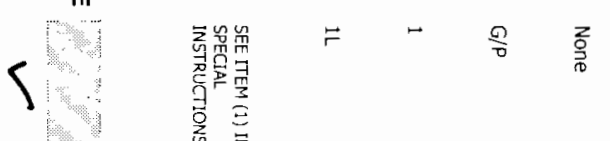
zy

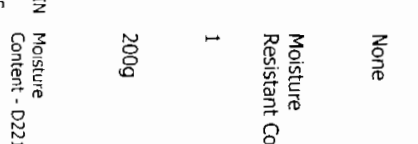
U $\dot{H} \stackrel{\substack{0 \\ \text { D }}}{=}$

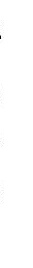

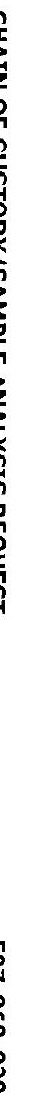

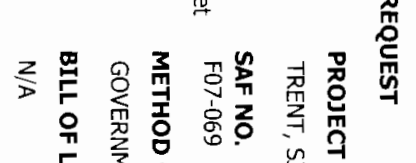

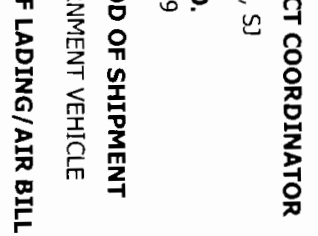

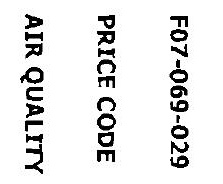

$\stackrel{\infty}{2}$

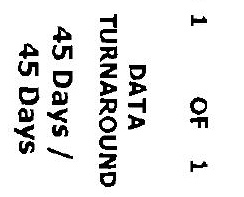




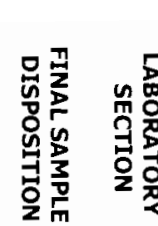

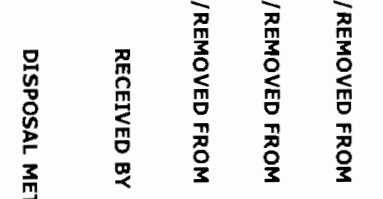

अं के
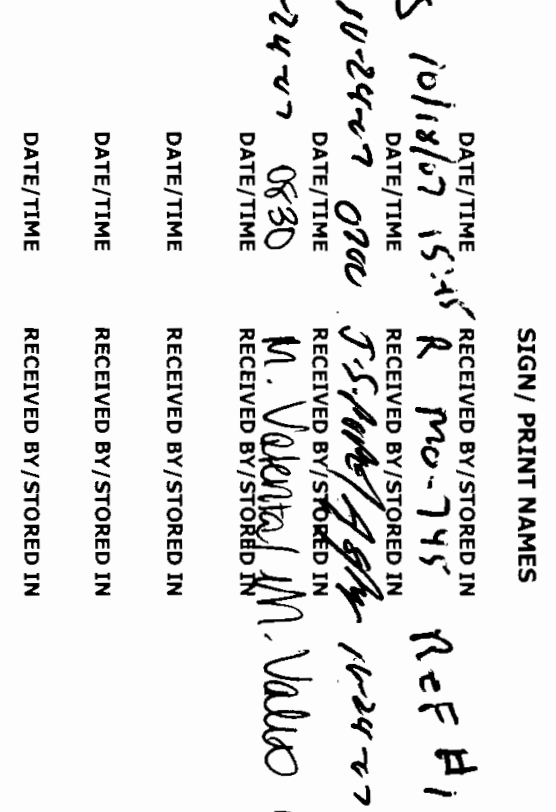

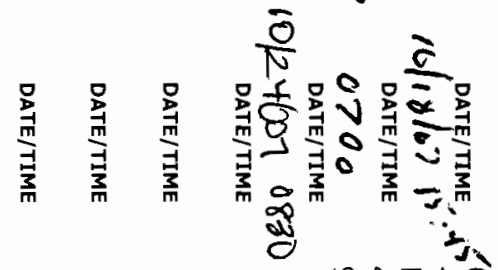

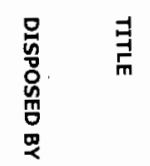

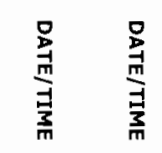

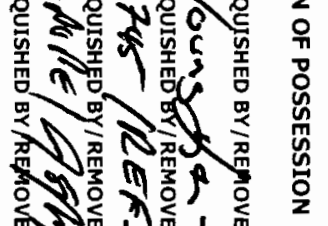

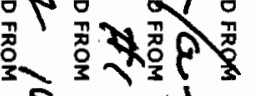
ई ई ई

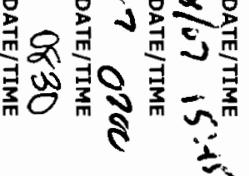

\section{黯}

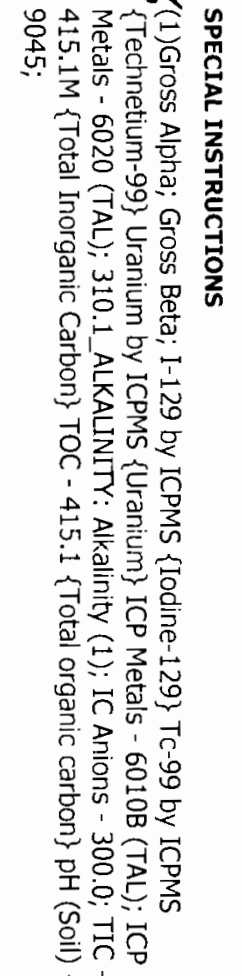

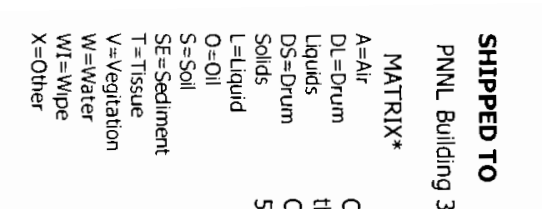

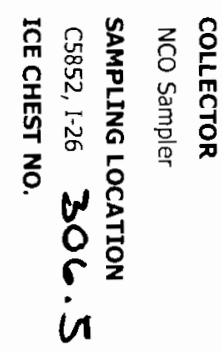

$\stackrel{c}{\circ}$

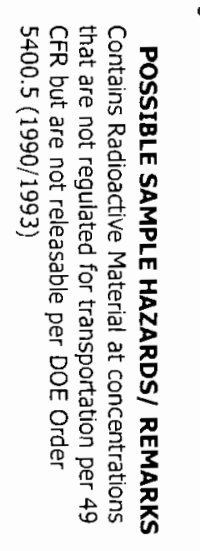

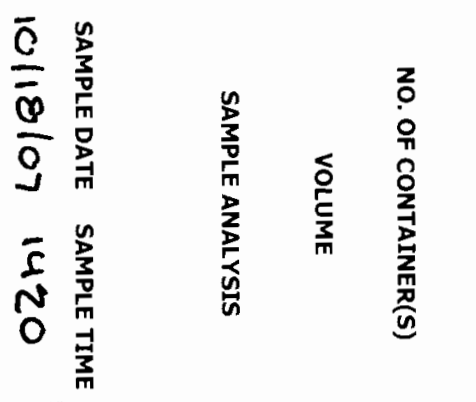

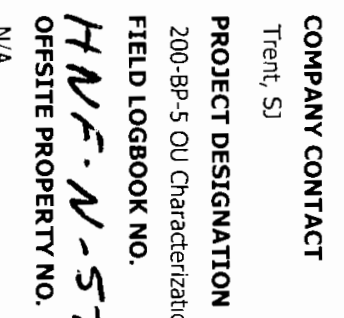

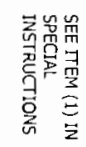

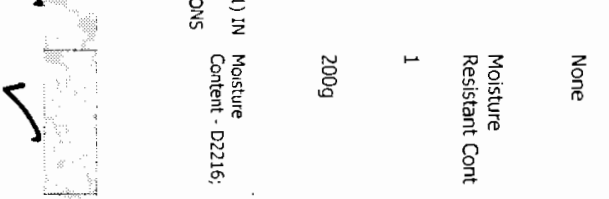

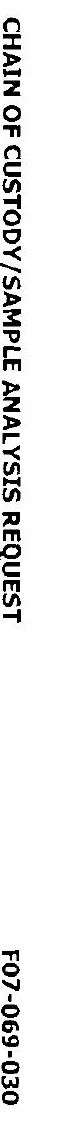

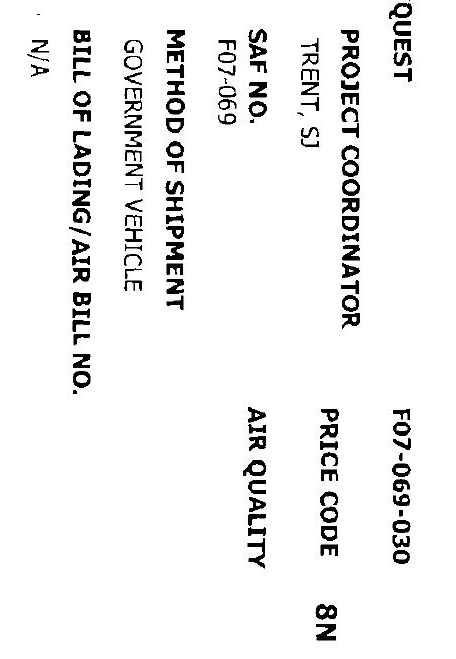




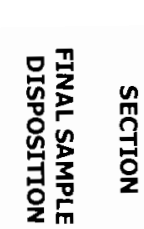

旁高

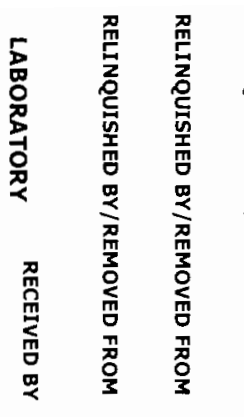
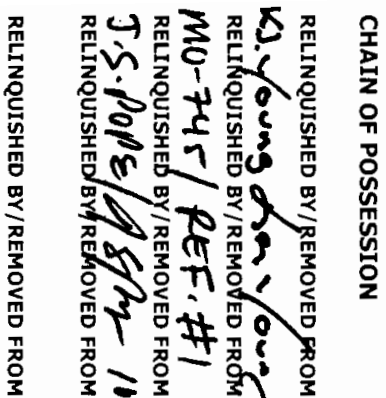

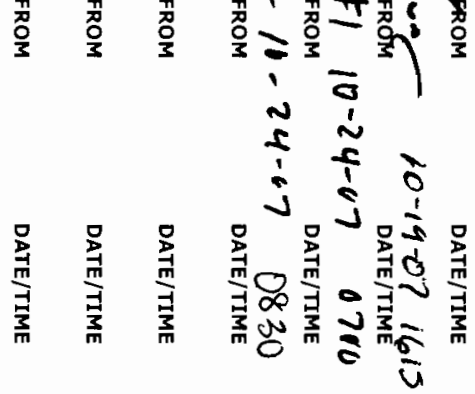
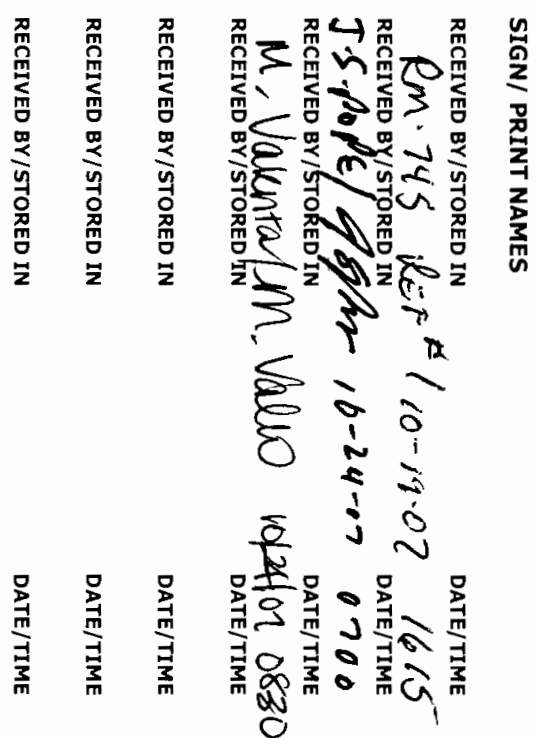

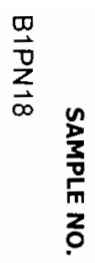

$\stackrel{g}{F}$

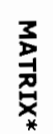

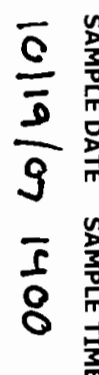

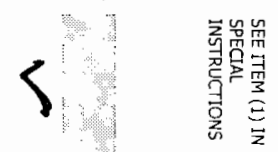

5

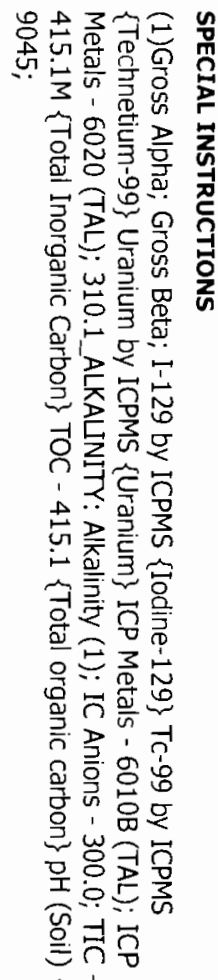

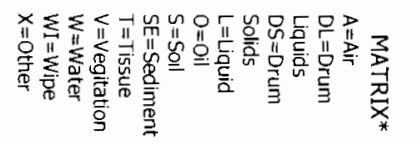

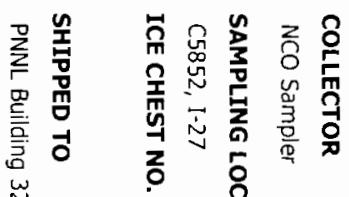

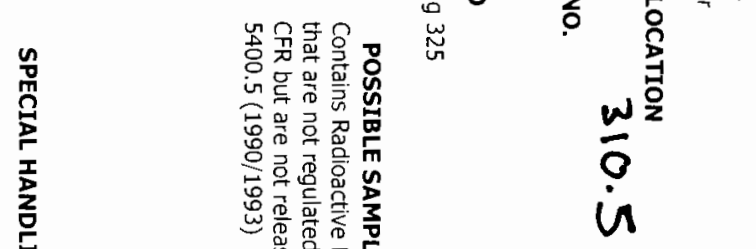

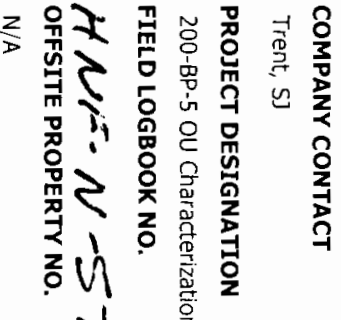

8 울

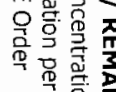

娄言

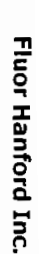

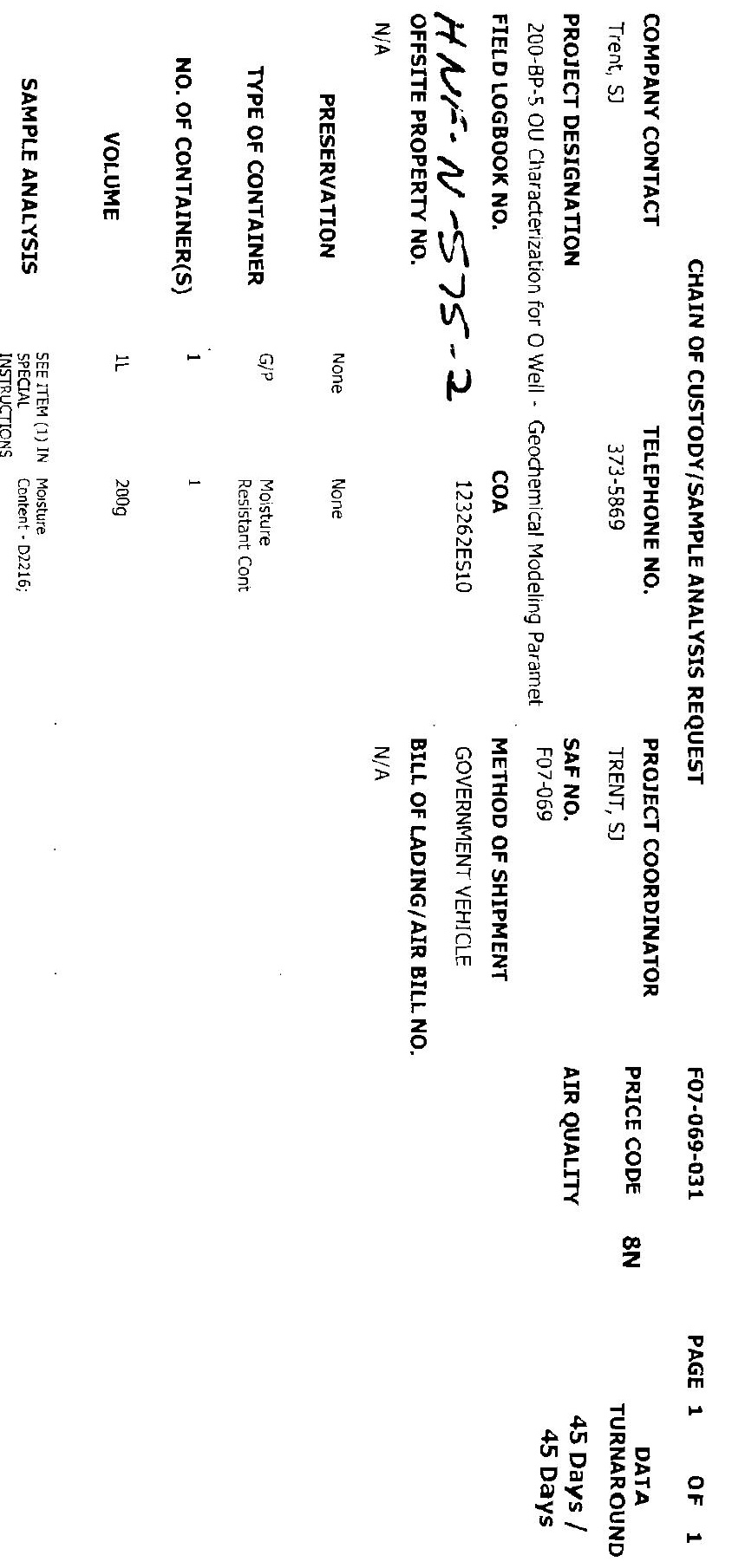




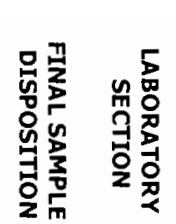

量

器

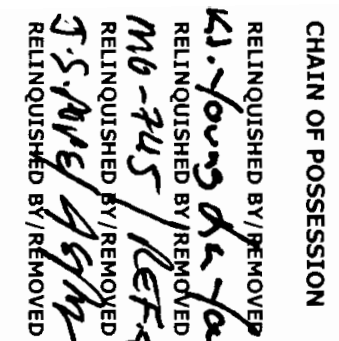

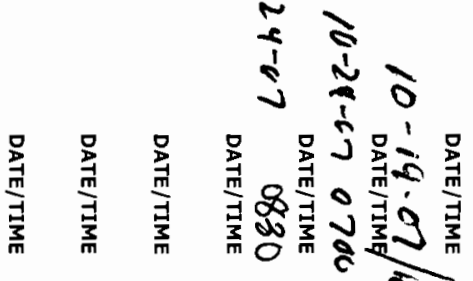
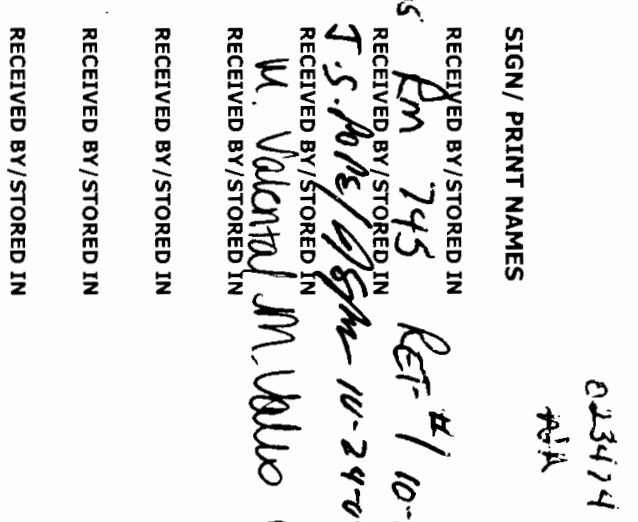

ह $j$

尊是

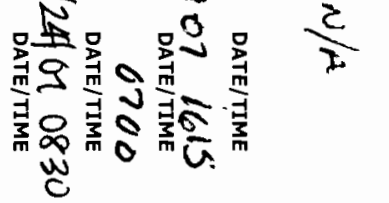<smiles>[CH-]C[I-]</smiles>

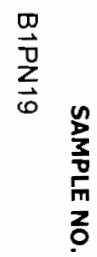

$\stackrel{\mathscr{O}}{ }$

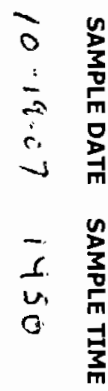

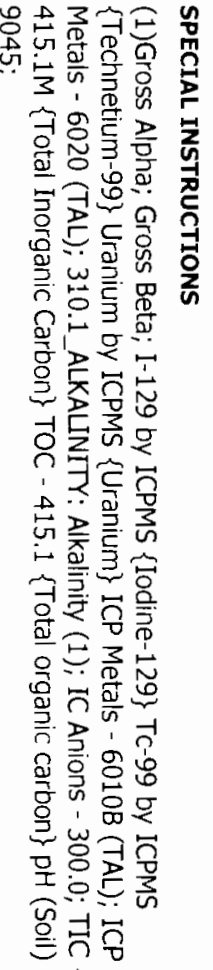

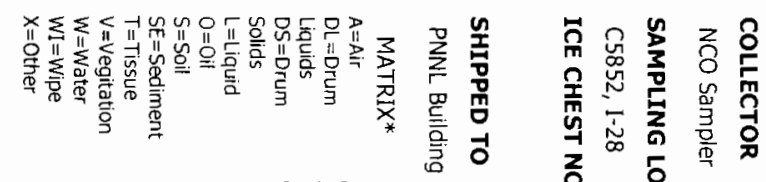
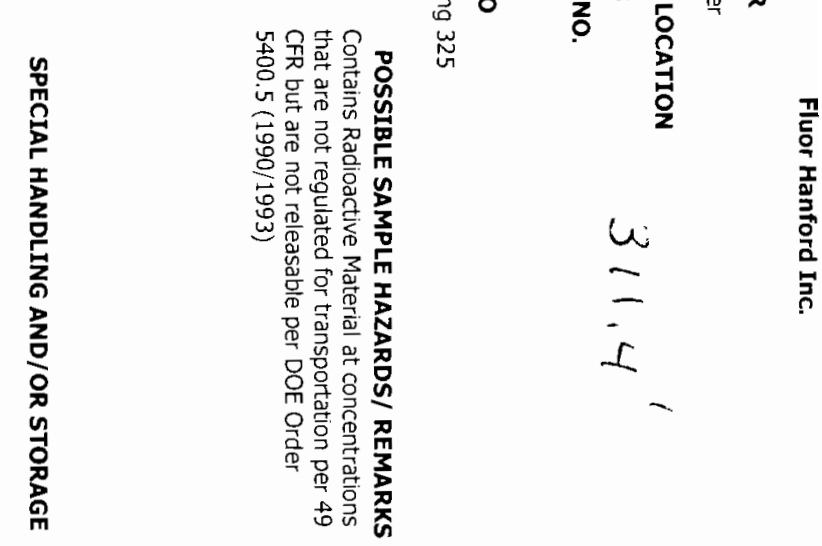

$c$

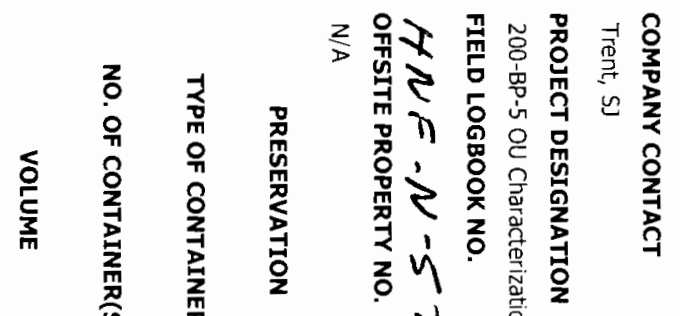

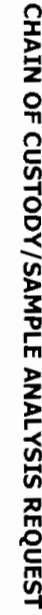
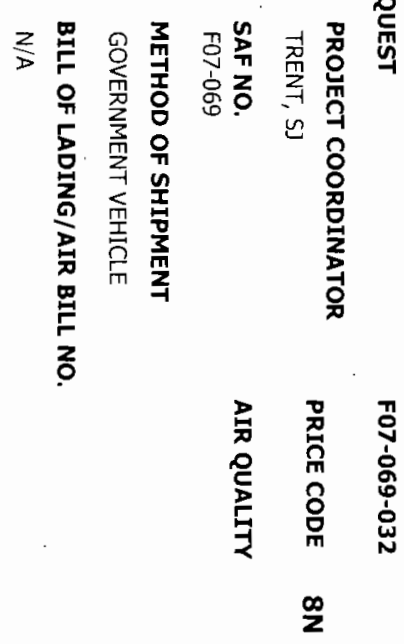

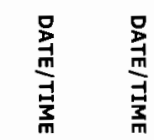




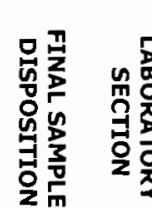

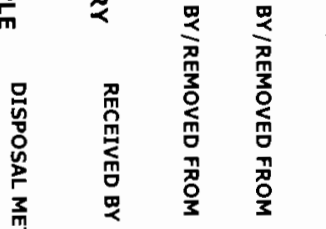

꽁

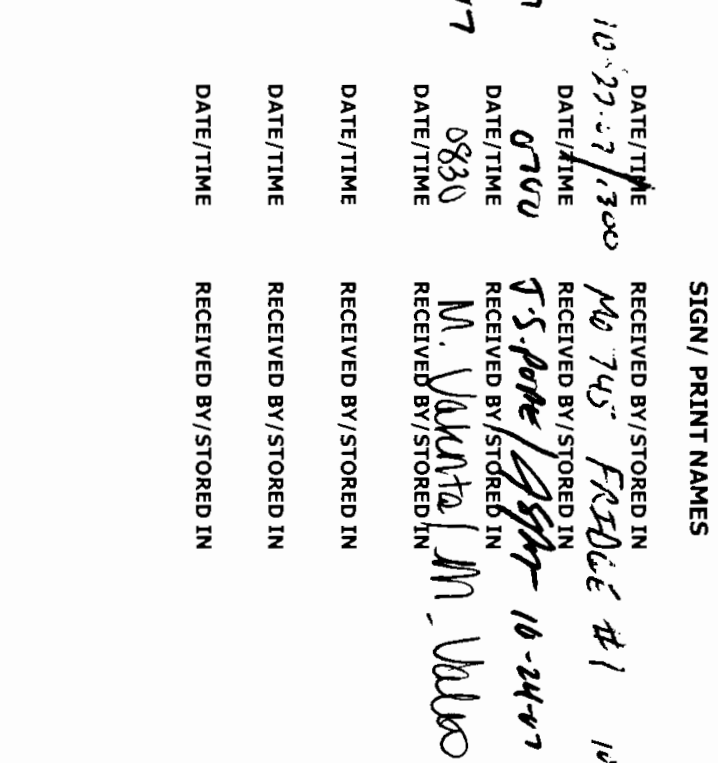

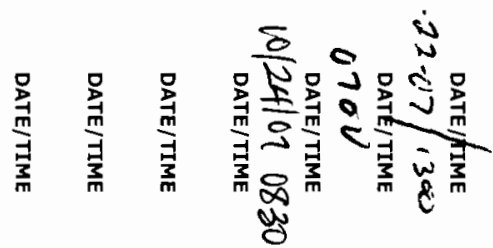

音

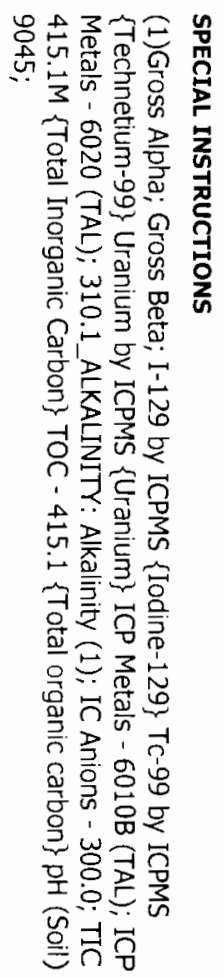

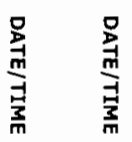

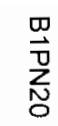

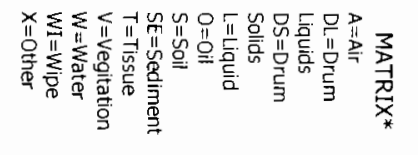

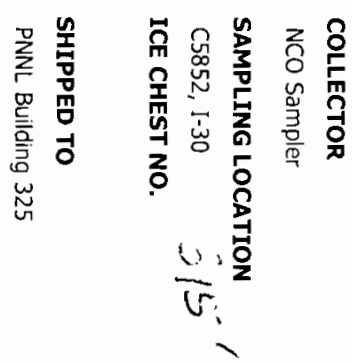

$\stackrel{\mathscr{O}}{F}$

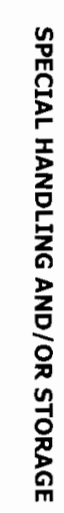

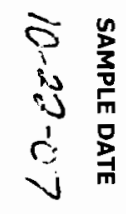

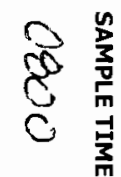

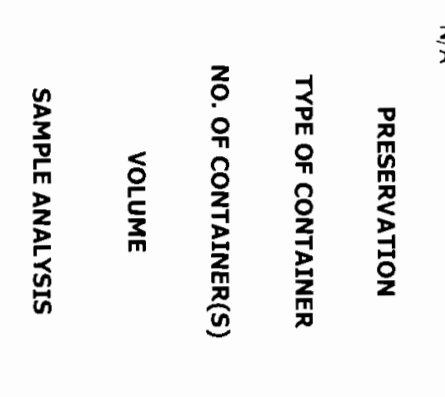

㢣

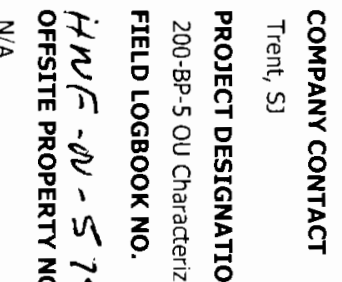

วั่

N $\stackrel{?}{\circ}$

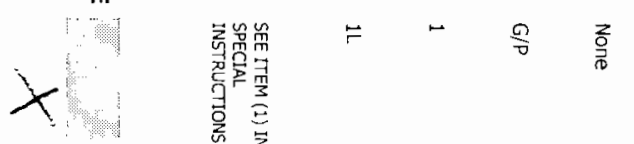

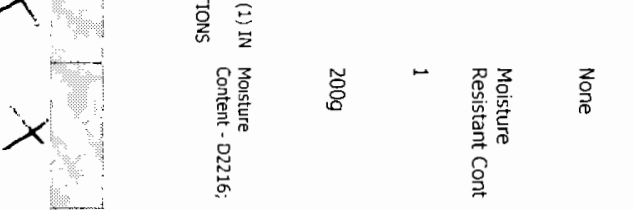

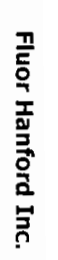

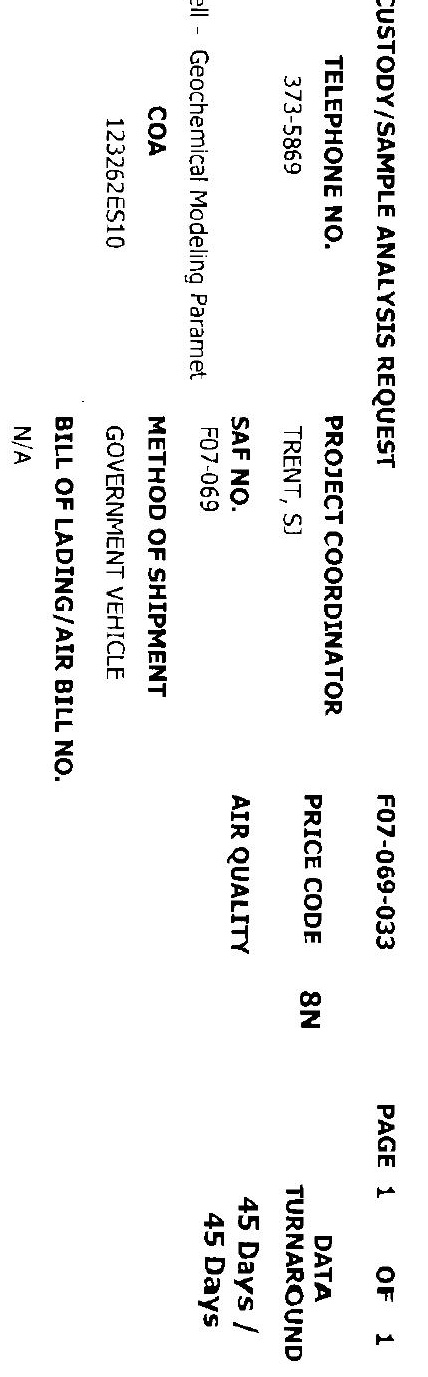




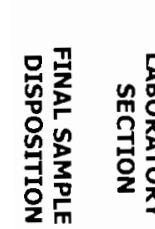

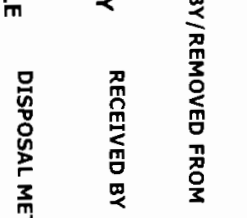
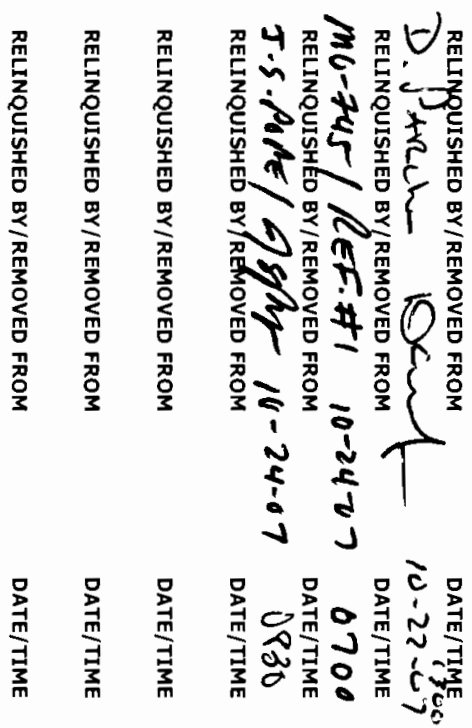

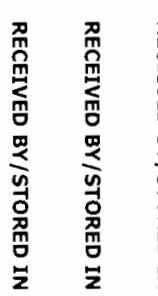

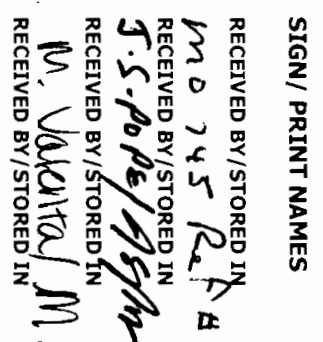

总 者

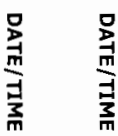

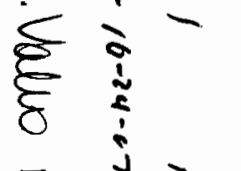

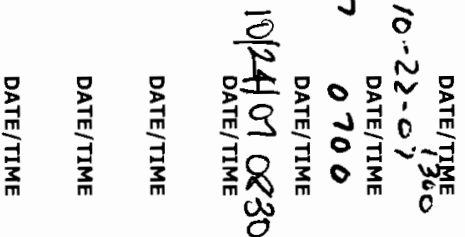

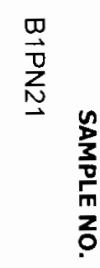

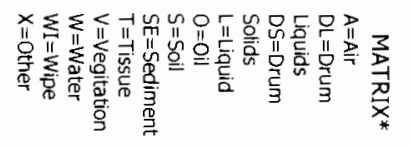

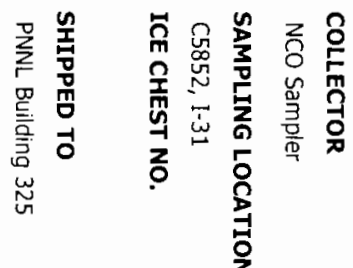

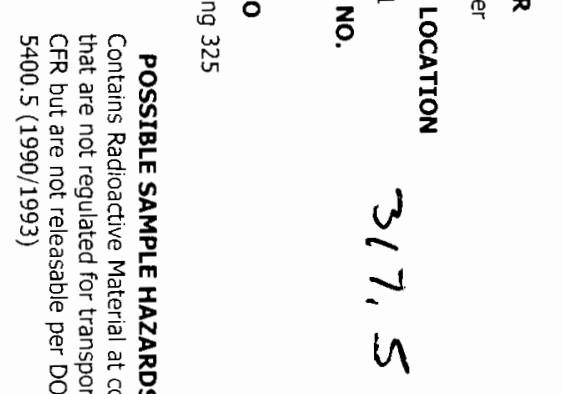

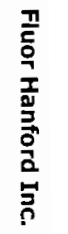

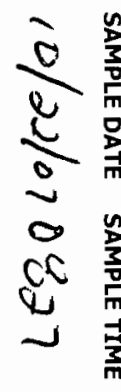

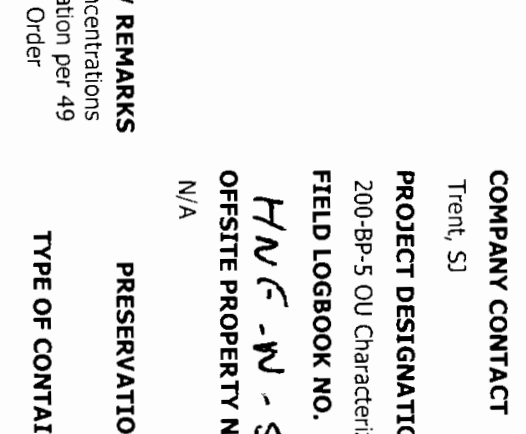

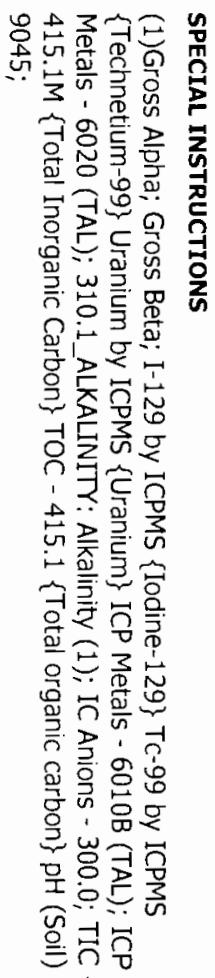

$\stackrel{\infty}{\stackrel{0}{r}}$

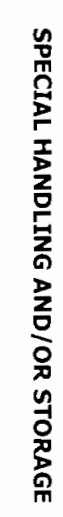

管

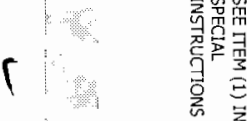

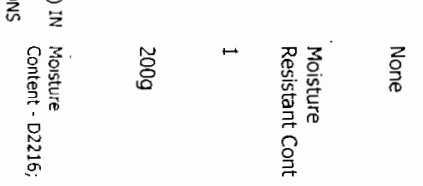

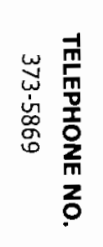

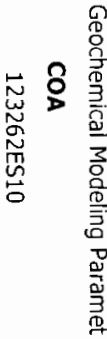

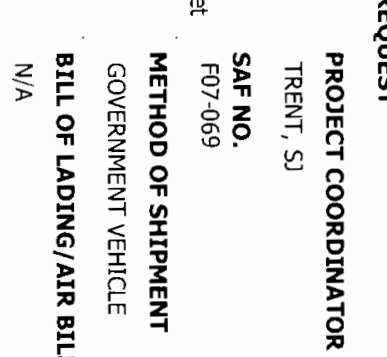

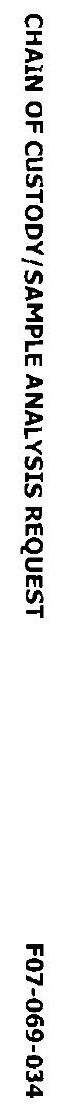

串
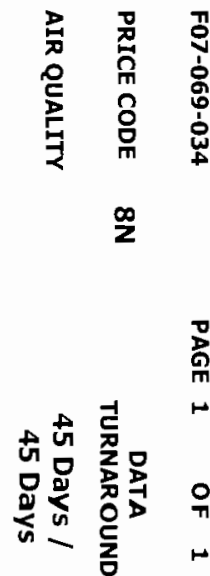


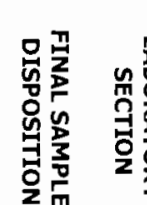

竞
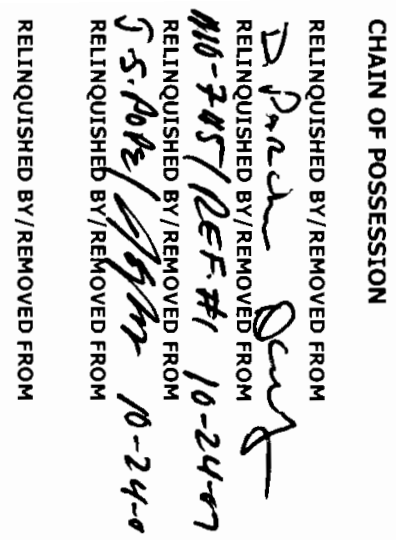

$\underset{\frac{2}{2}}{\frac{2}{m}}$
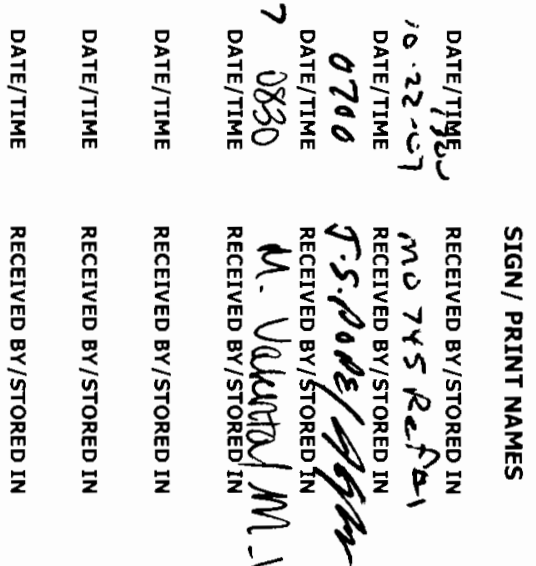

$$
\text { 瓷 }
$$

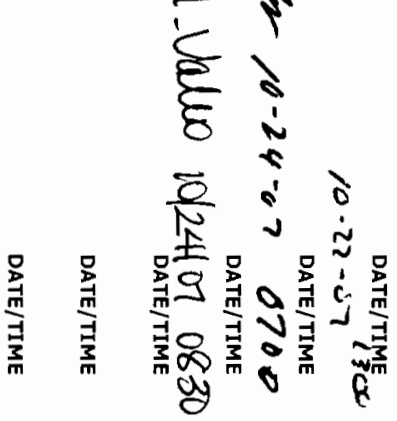

$\stackrel{\mathfrak{O}}{=}$
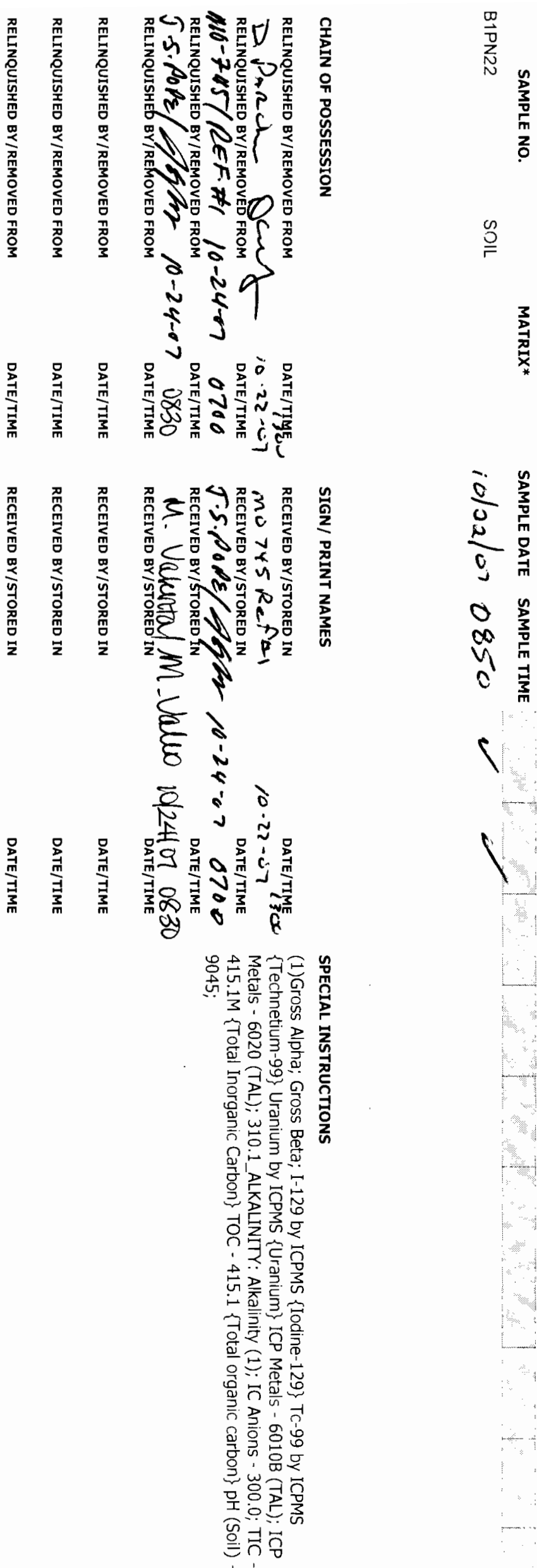

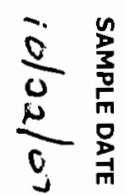

$\begin{array}{ll}0 & n \\ \infty & \frac{3}{3} \\ 0 & \frac{\pi}{\pi} \\ 0 & \frac{7}{3}\end{array}$

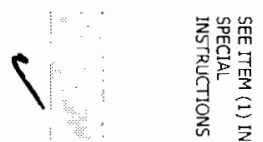

$\frac{0}{\pi} i$ i

猽

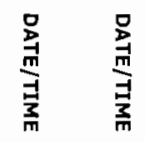

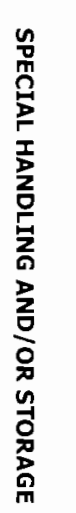

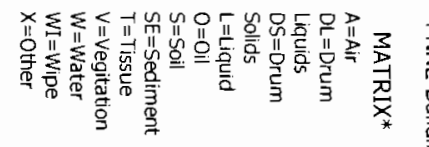

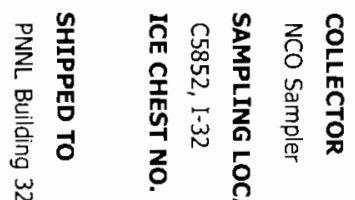

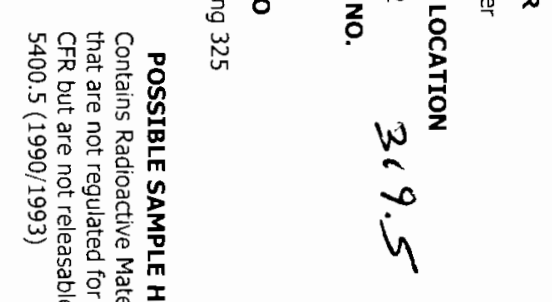

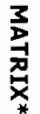

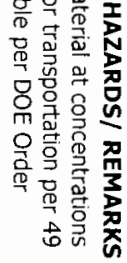

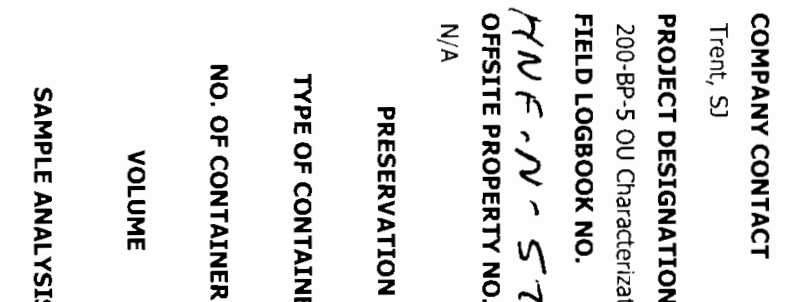




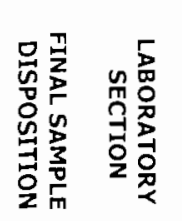

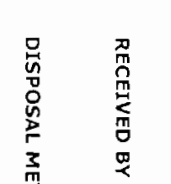

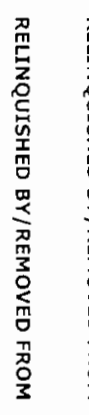

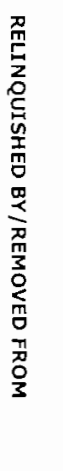

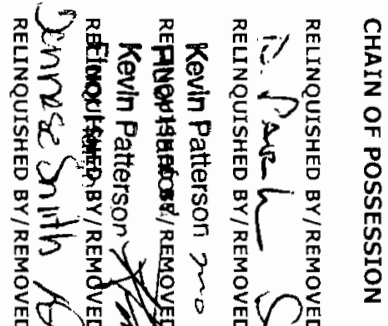

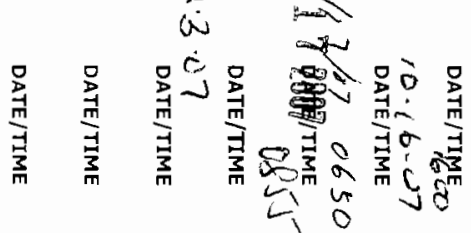

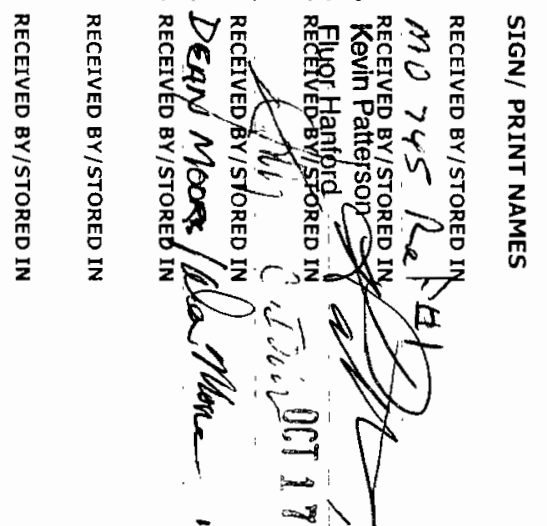

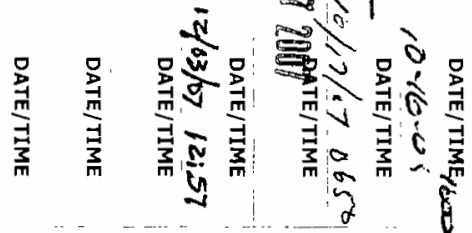
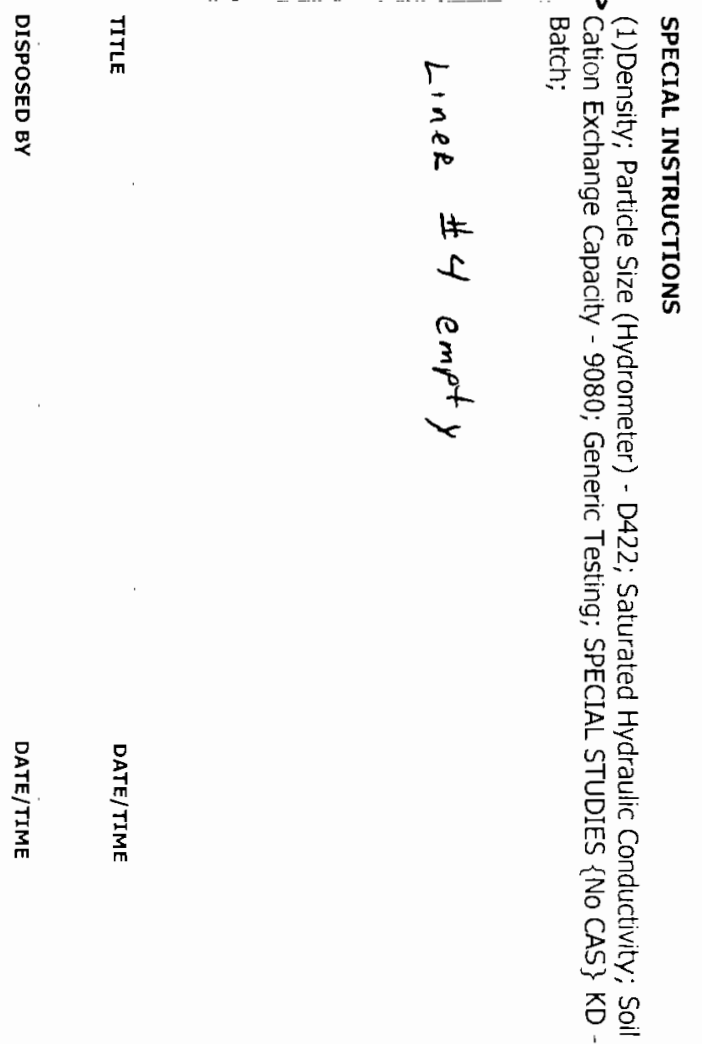

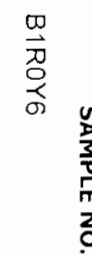

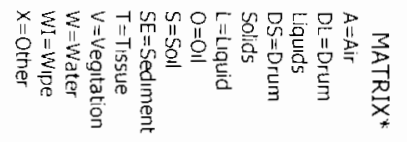

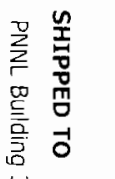

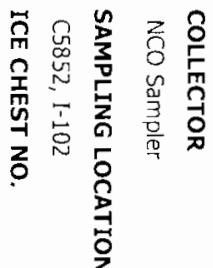

$\frac{\infty}{O}$

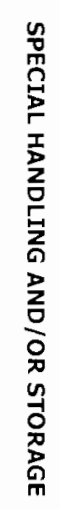

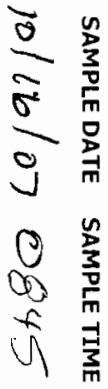

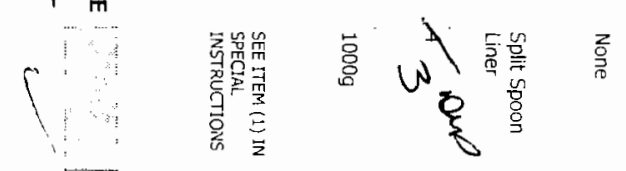

$z$

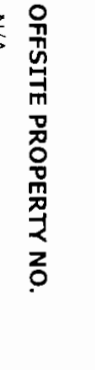

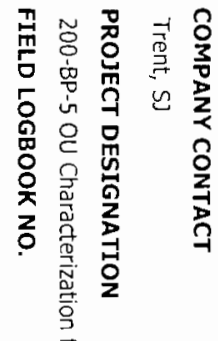

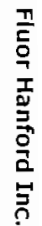

or

$v$

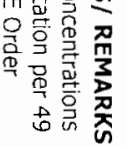

4

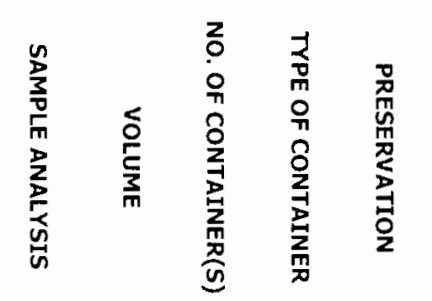<smiles>CCC</smiles>

愛

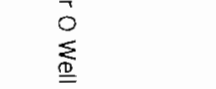

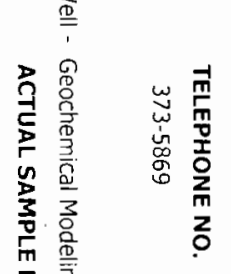

品

举

궁

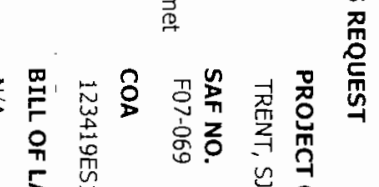

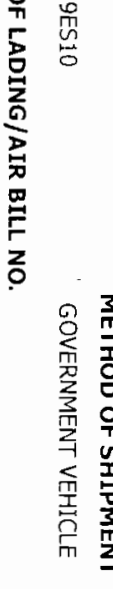

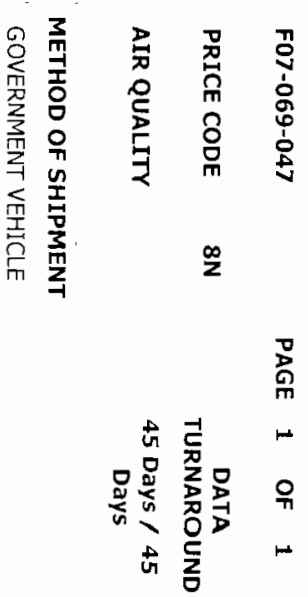




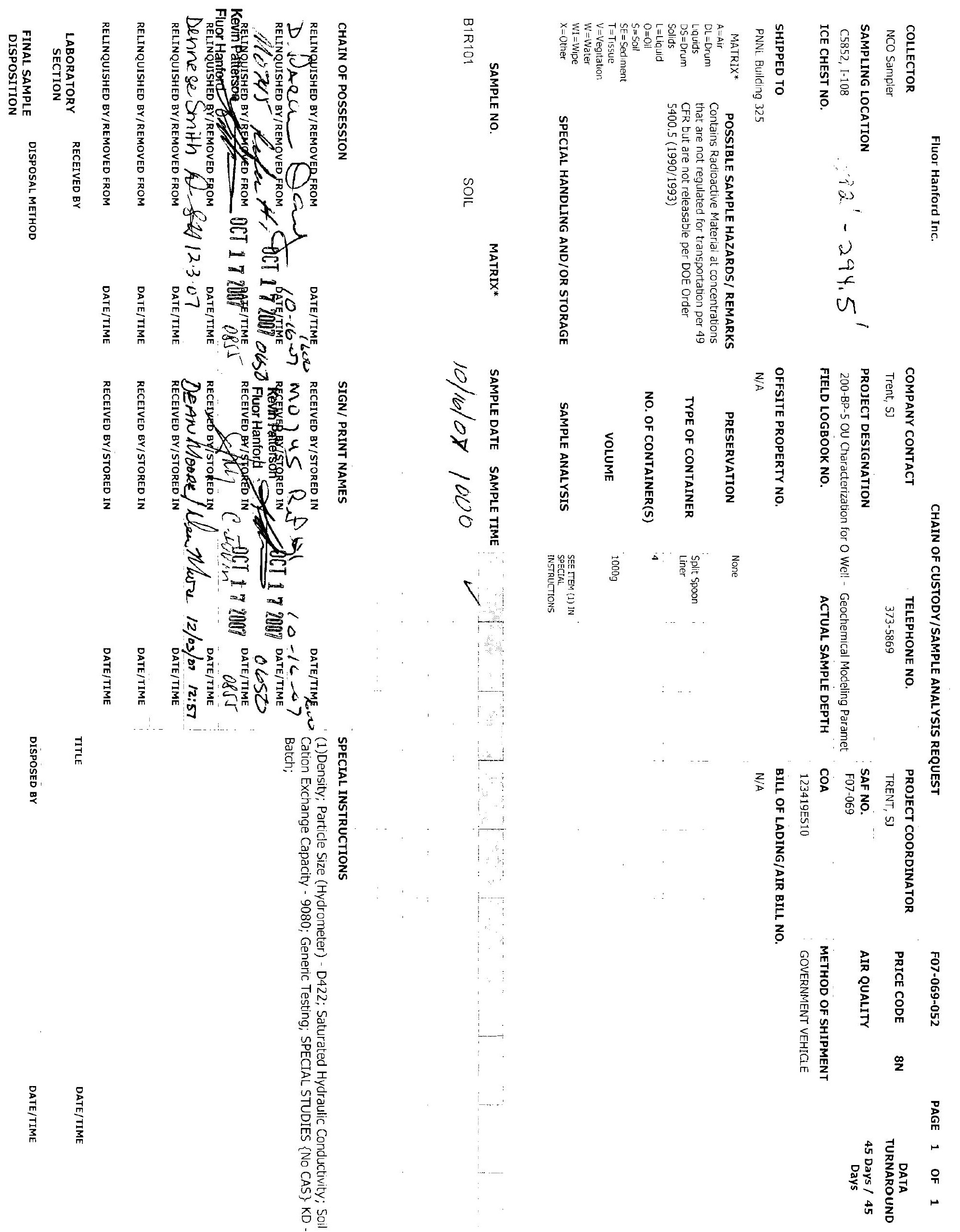




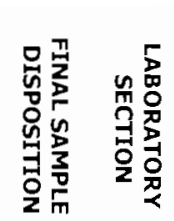

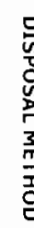

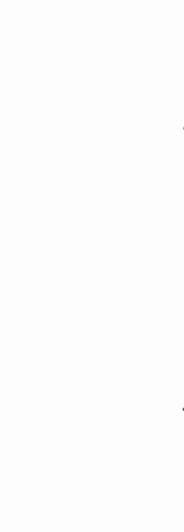

言<smiles>[14CH]1=[14CH][14CH]=[14CH]1</smiles>

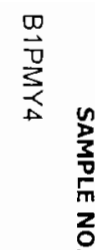

$\stackrel{0}{\stackrel{0}{ }}$

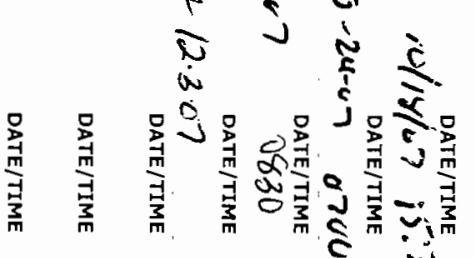

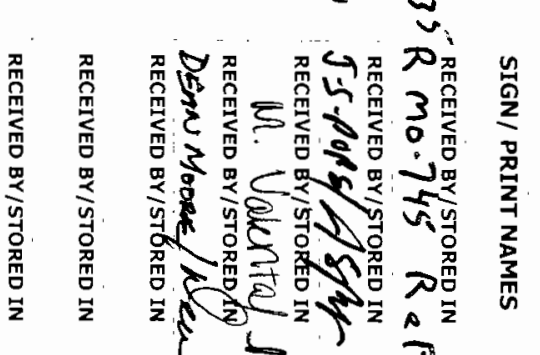

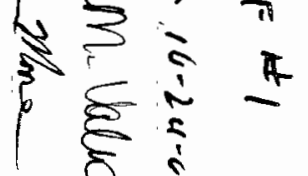

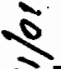

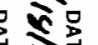

啇

w

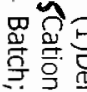

㽚器

语

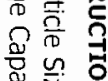

芦离

嗃

寄

D.

足

岕

婴高

폰

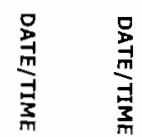

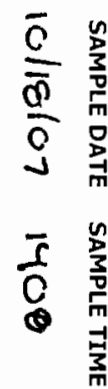

$\zeta$

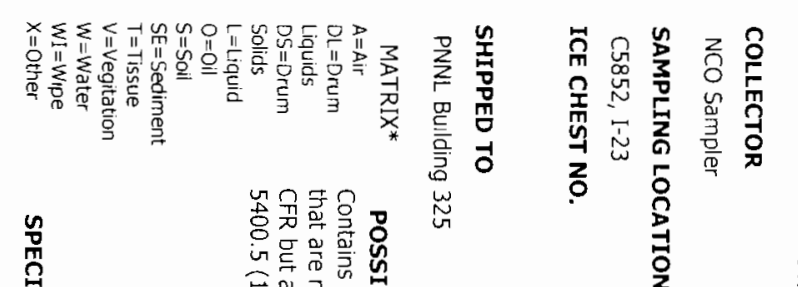

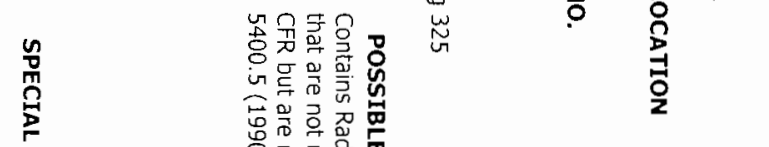

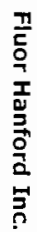

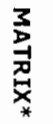

总

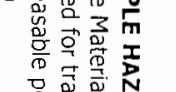

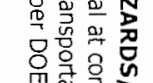

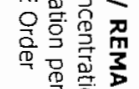

古空

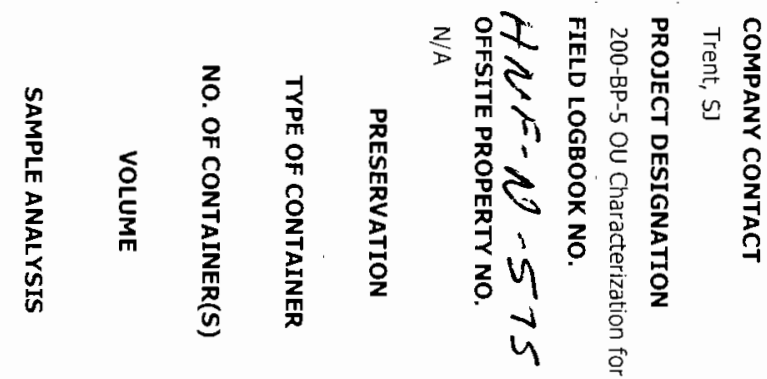

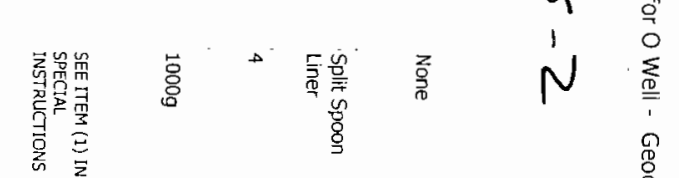

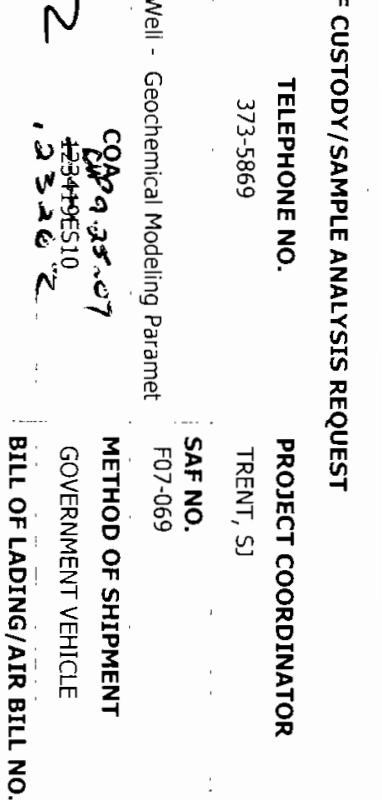

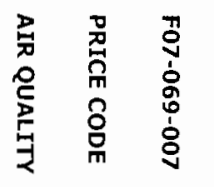

$\stackrel{\infty}{2}$

ग

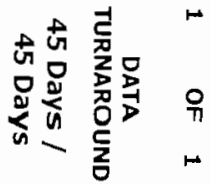




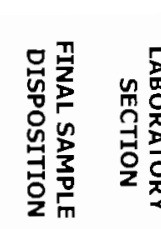

屖

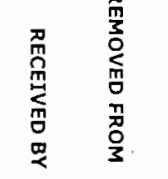

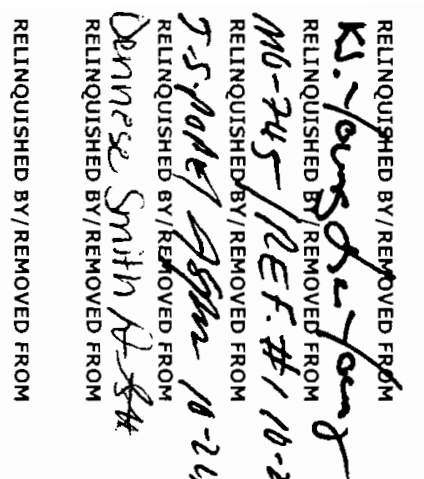

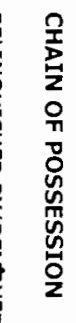

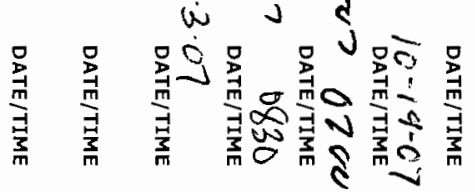
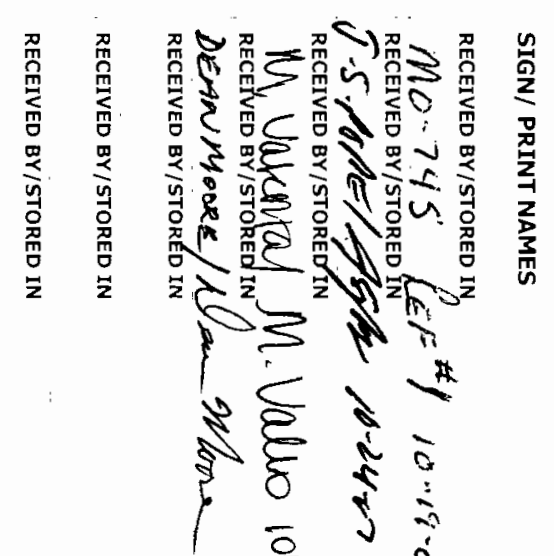

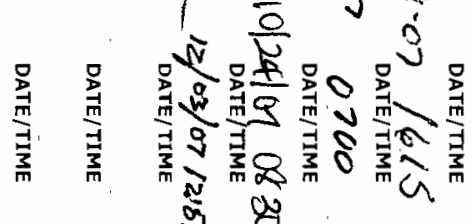

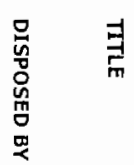

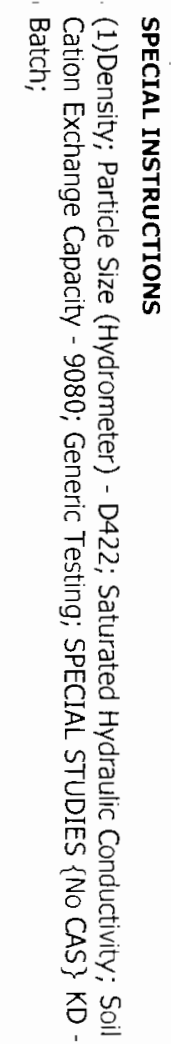

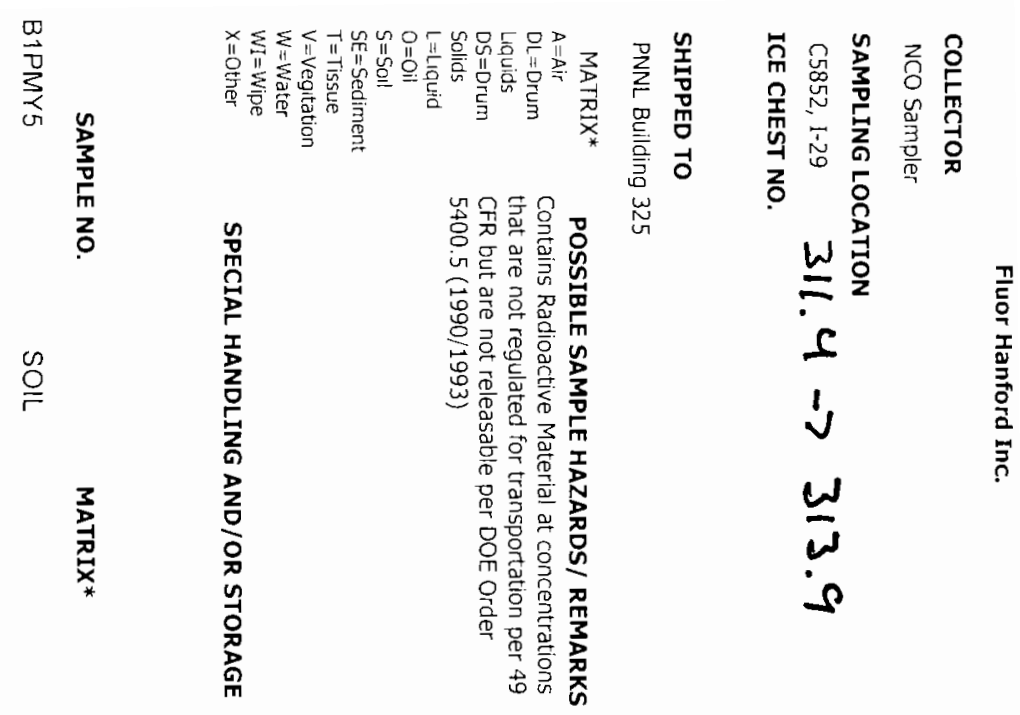

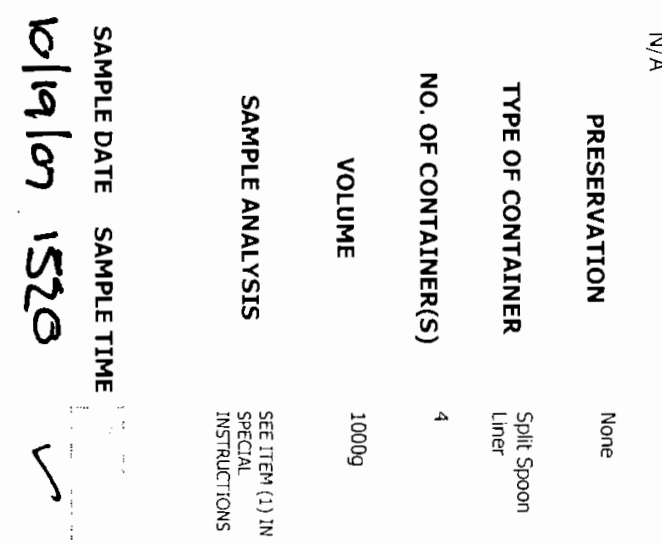

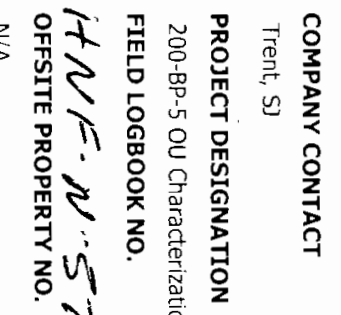

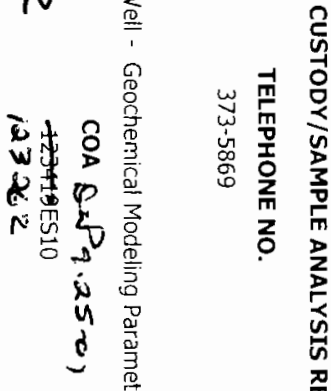

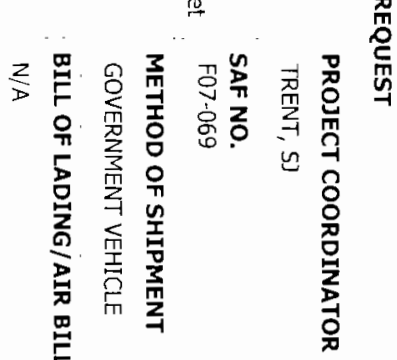

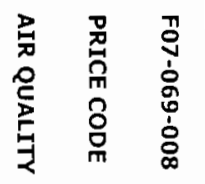

$\stackrel{\infty}{2}$

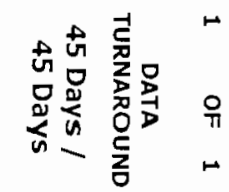




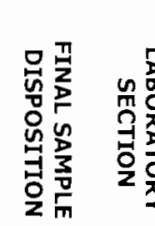
资高
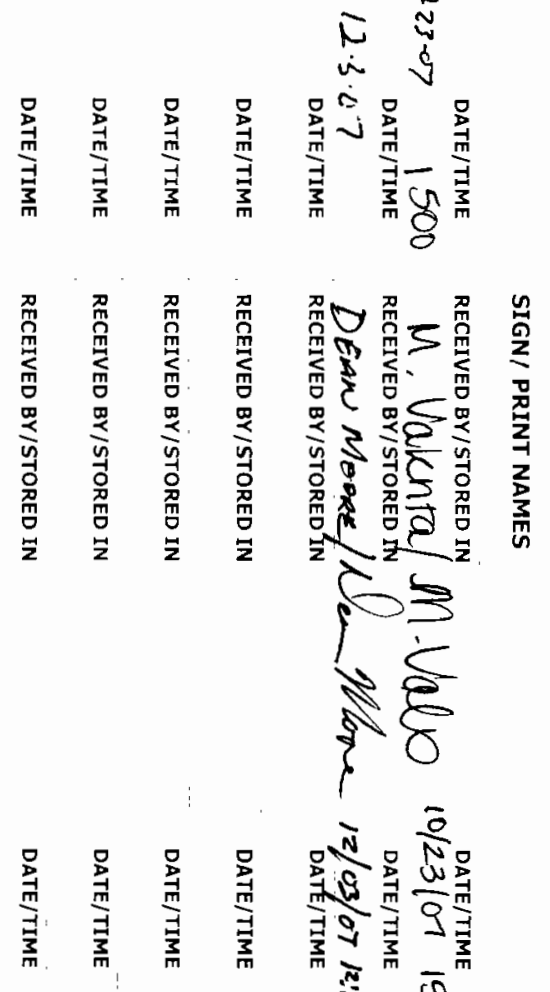

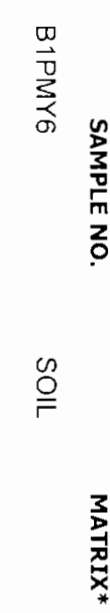

$\bar{d}$
$w$
1
$\dot{v}$

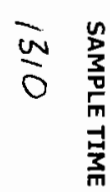

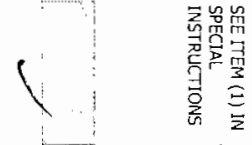

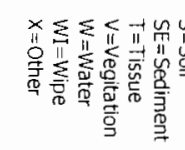

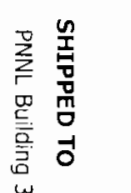

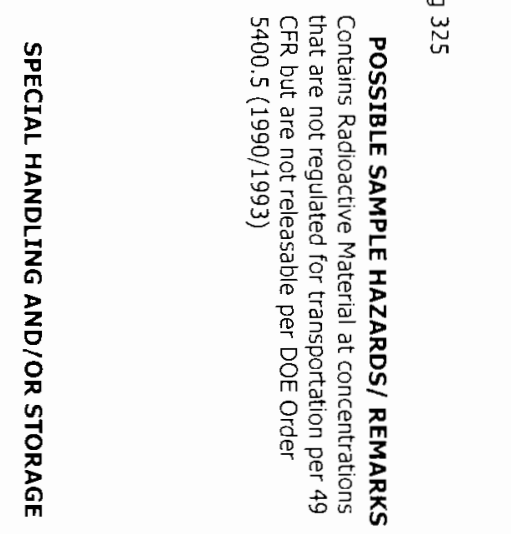

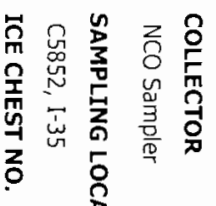

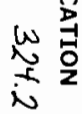

a



$\infty$

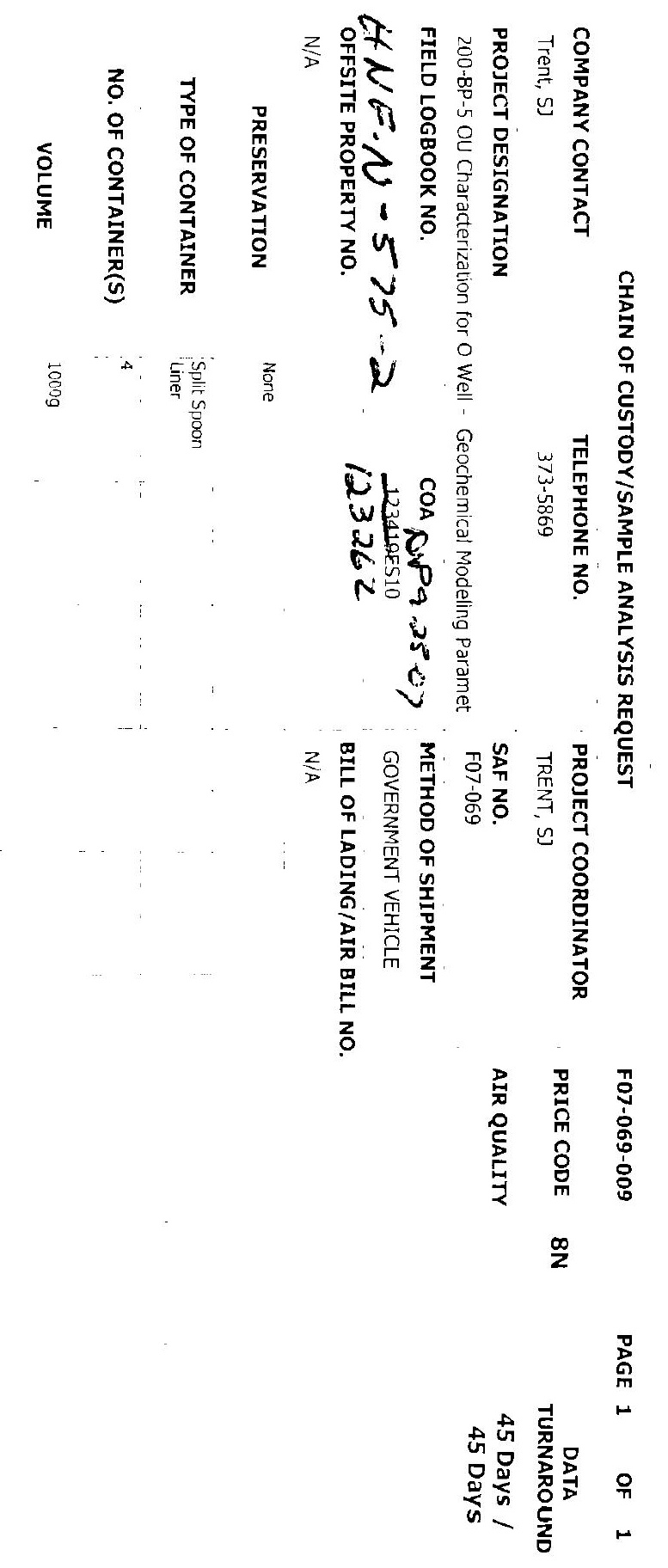




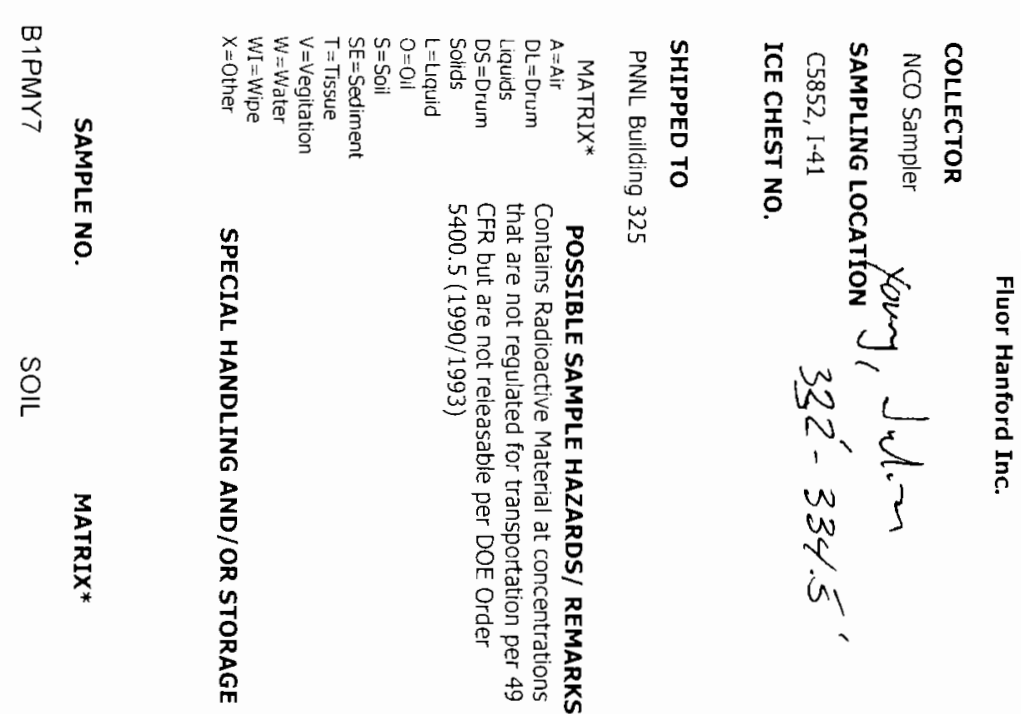

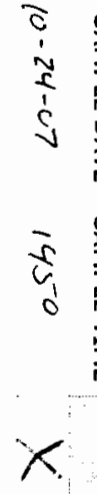

녹육

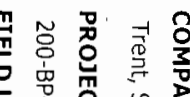

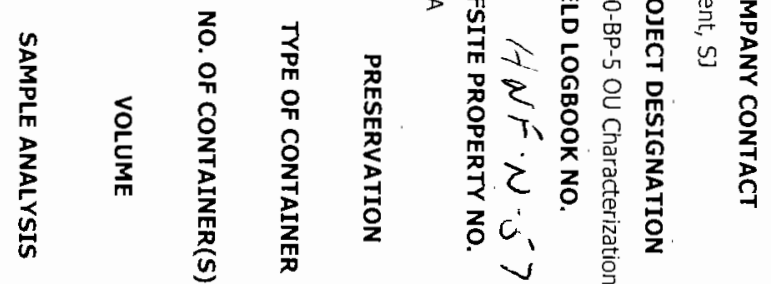

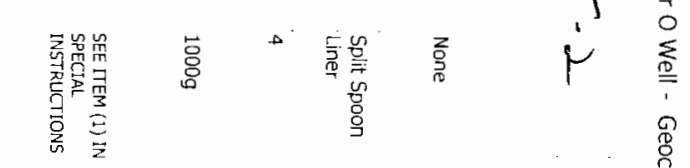

올

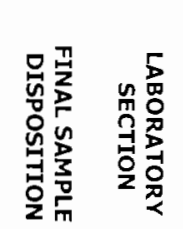

$: \frac{\overline{2}}{\frac{2}{2}}$

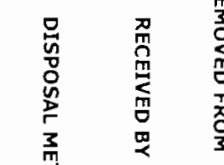
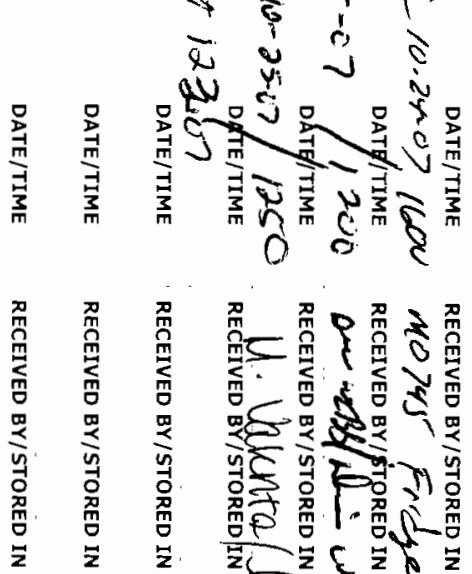

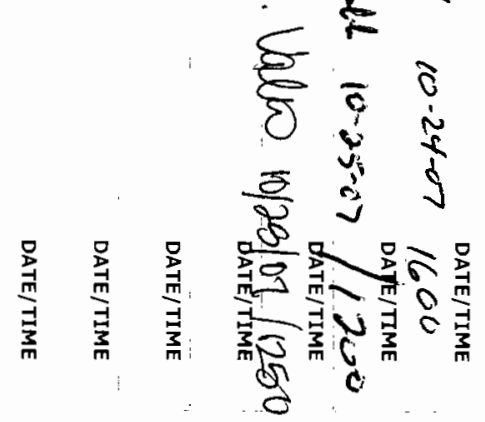

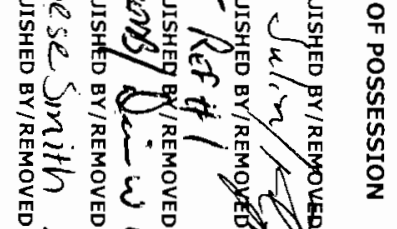

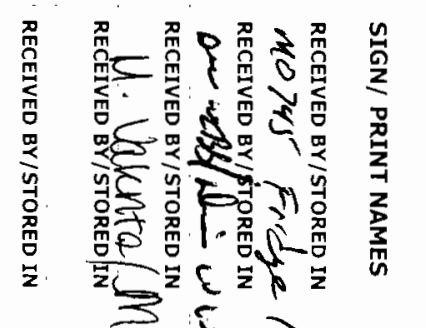

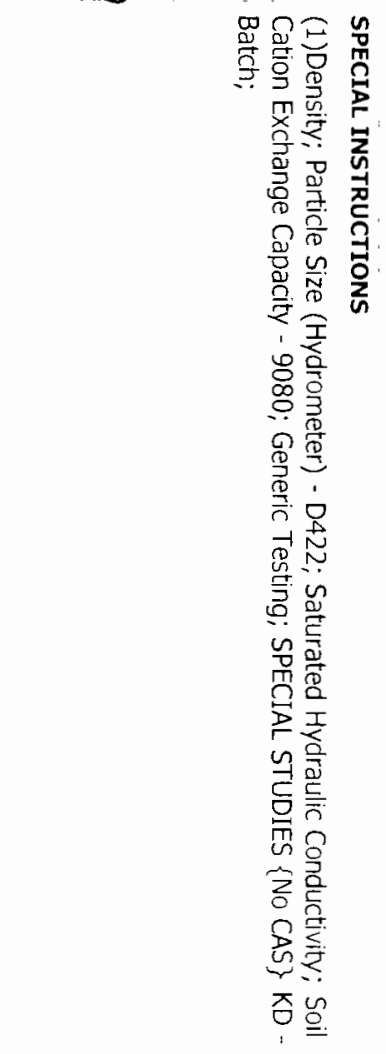

1
量 素
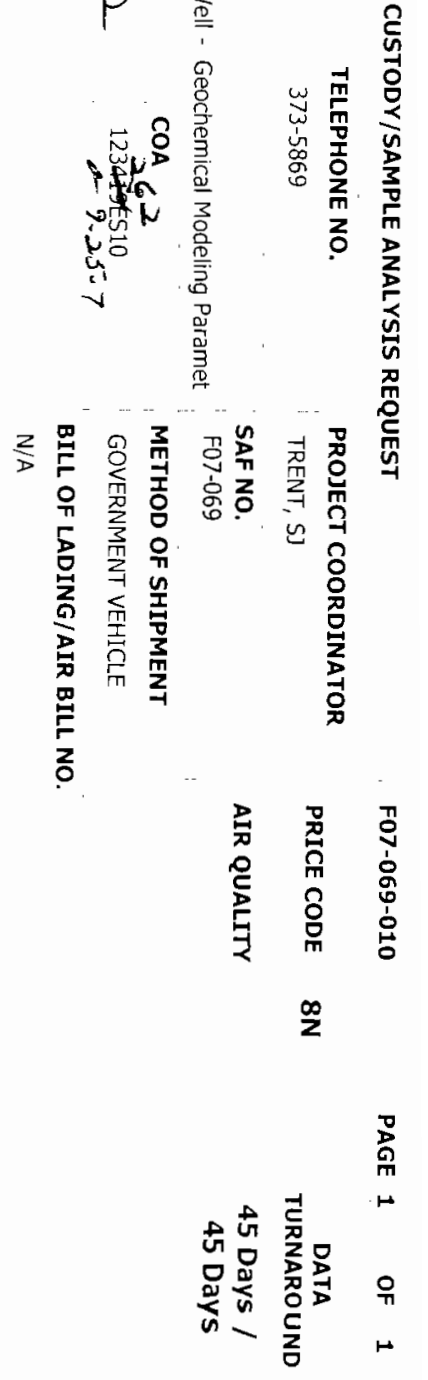
$\|$

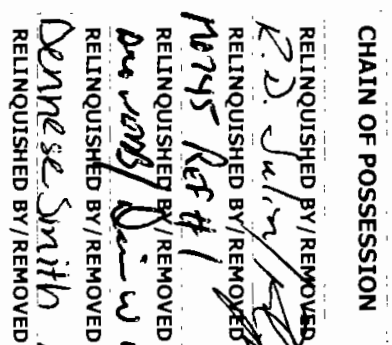

$\frac{2}{\frac{2}{2}}$
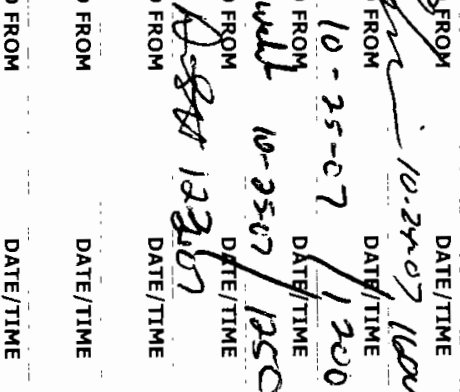

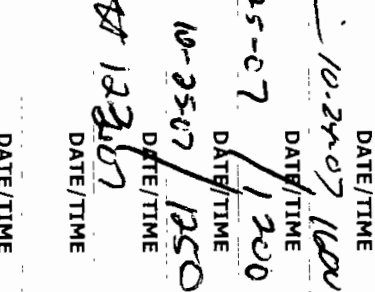
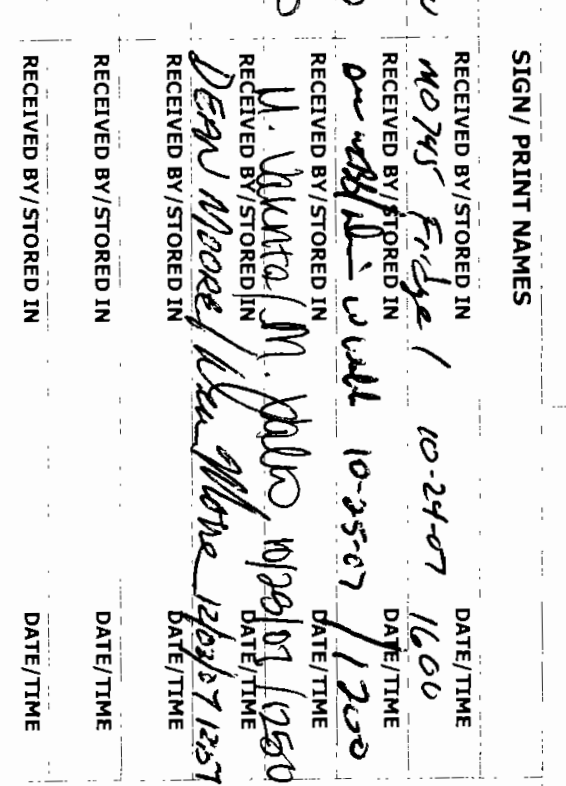

言

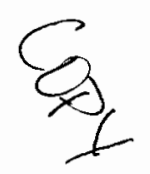

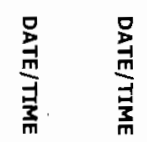
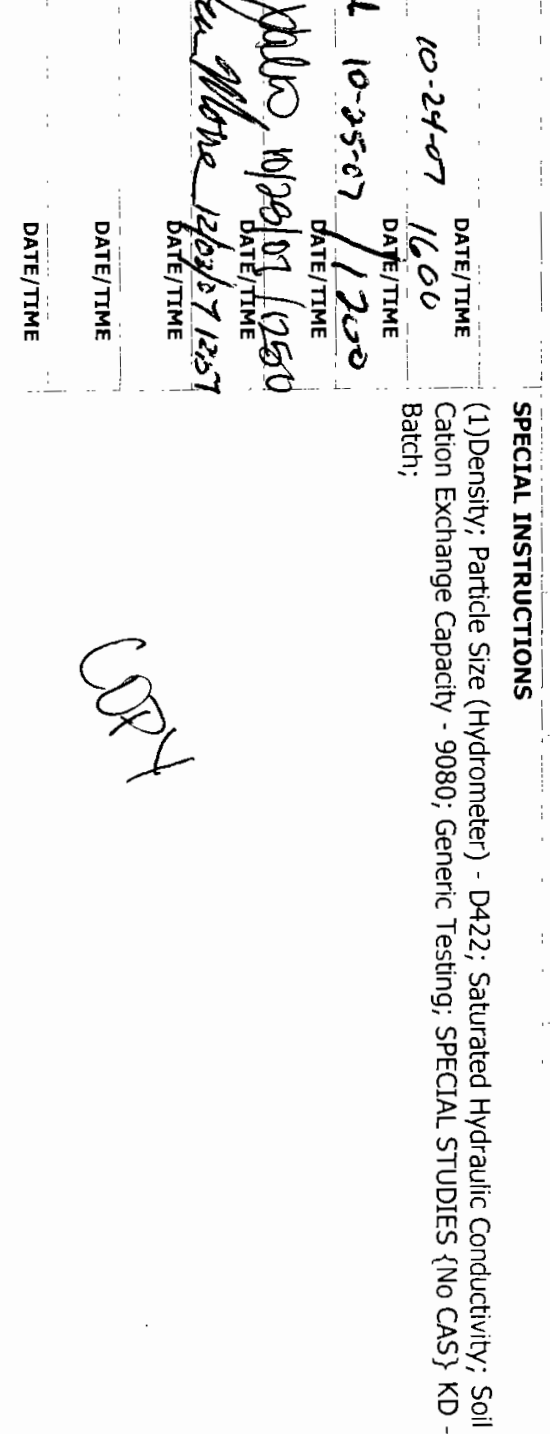

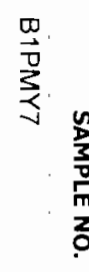

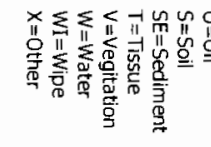

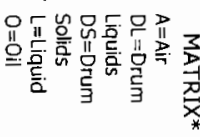

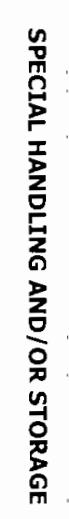

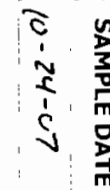

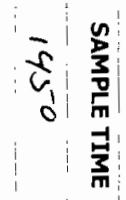

$\times$

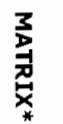

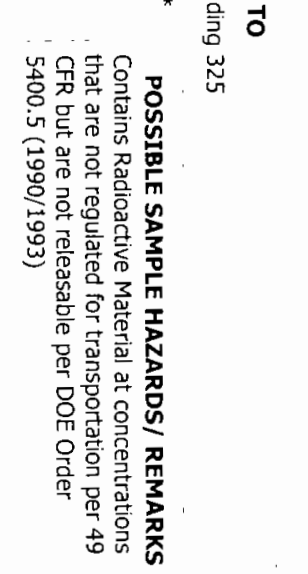

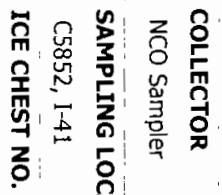

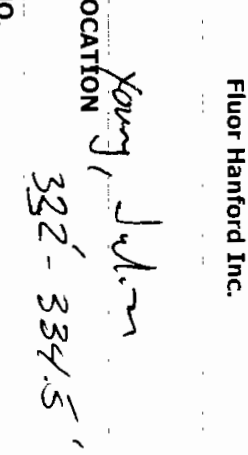

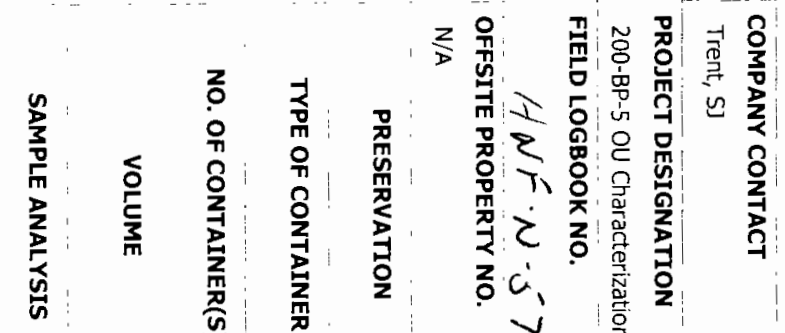

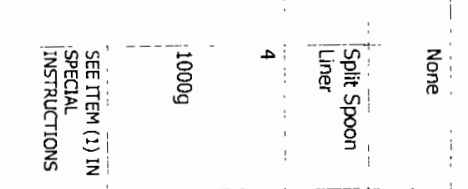

$$
\text { M }
$$

i $\sum_{i=\frac{0}{1}}^{0}$

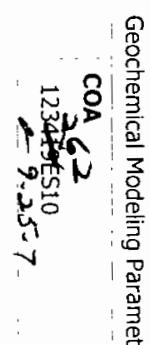

z

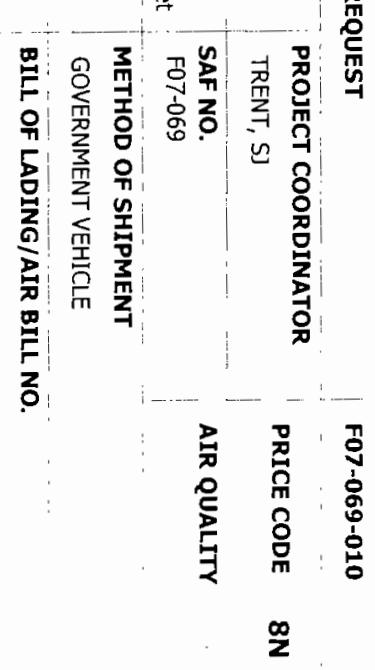

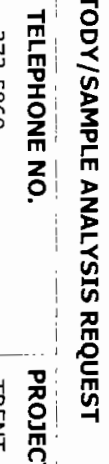
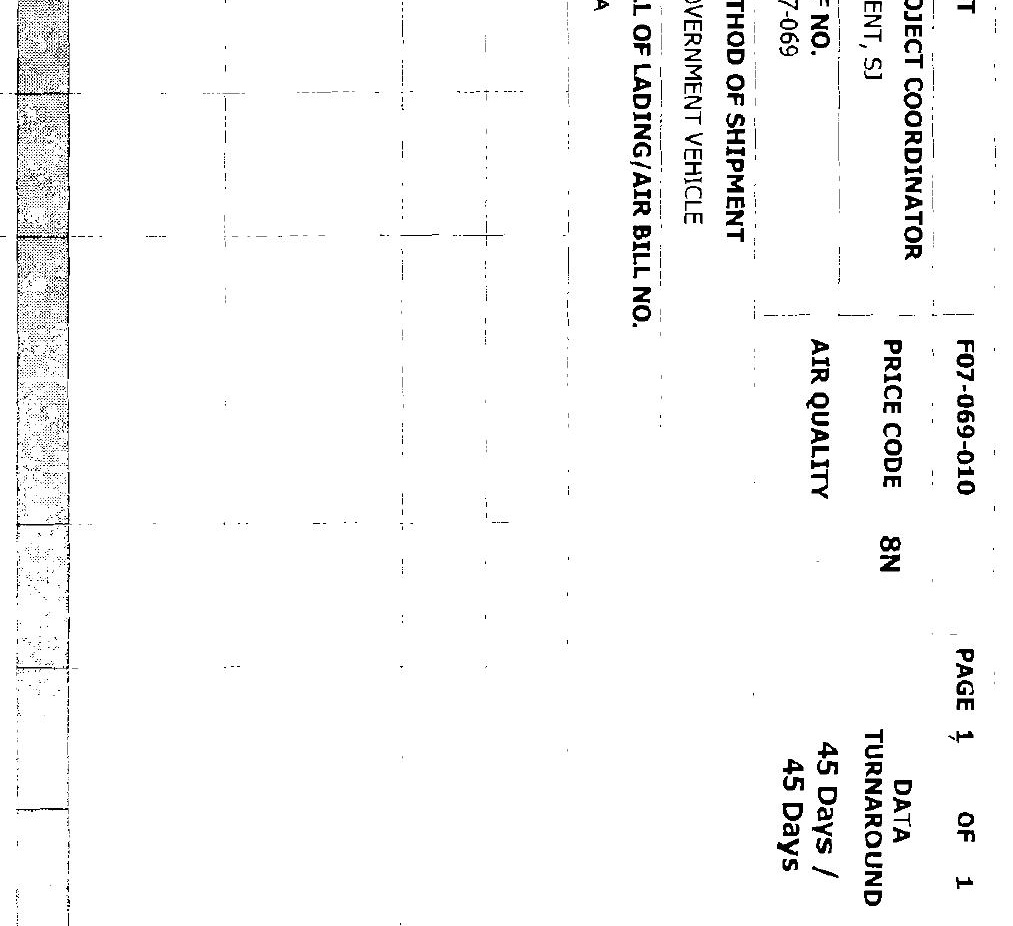


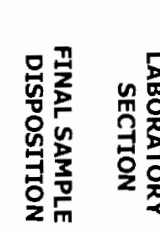
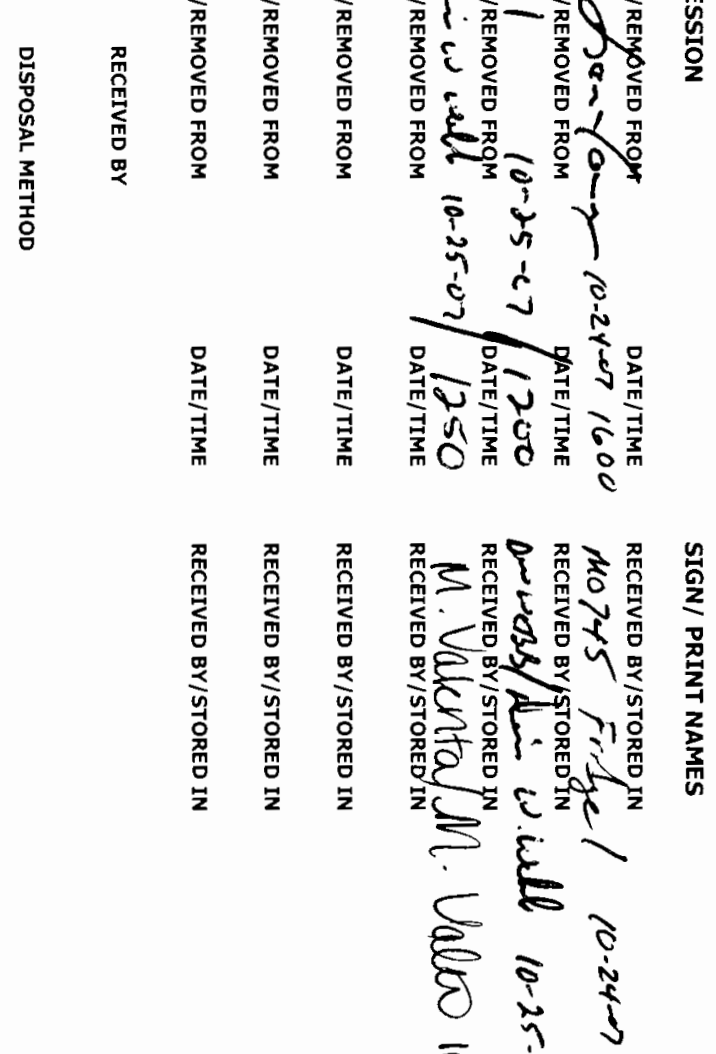

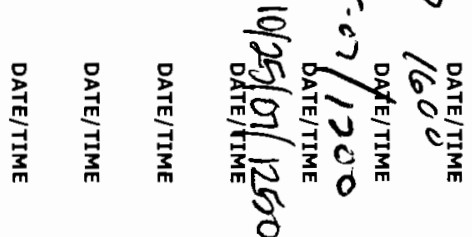

$\underset{m}{\vec{n}}$

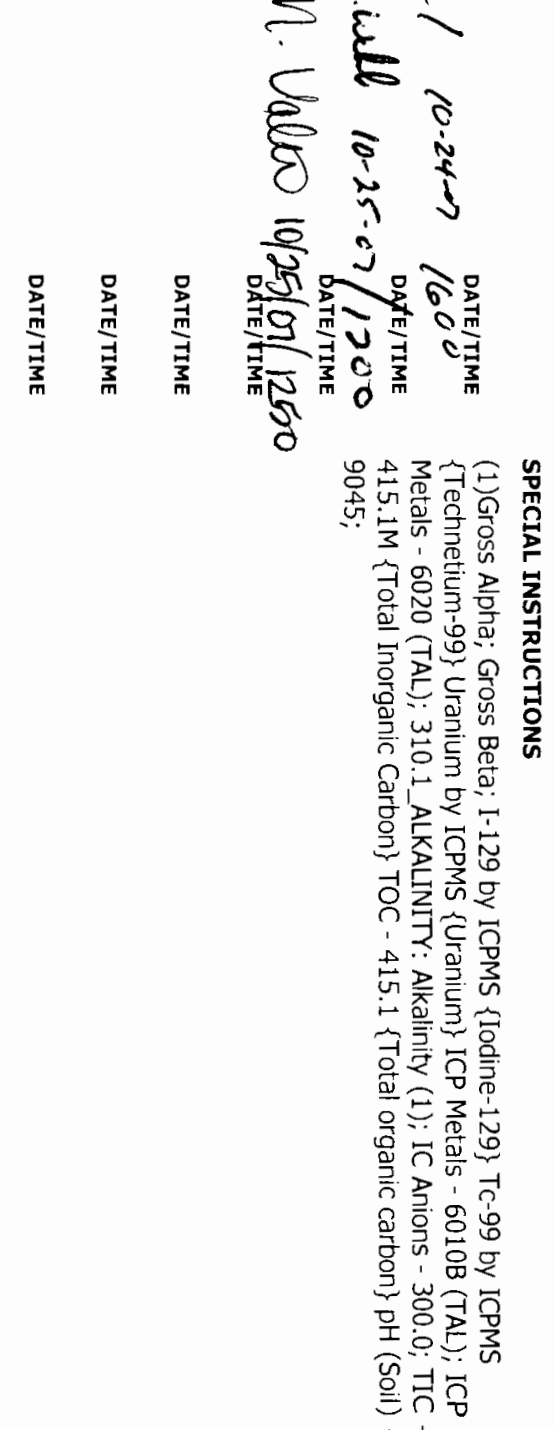

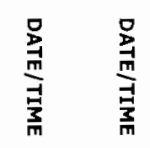

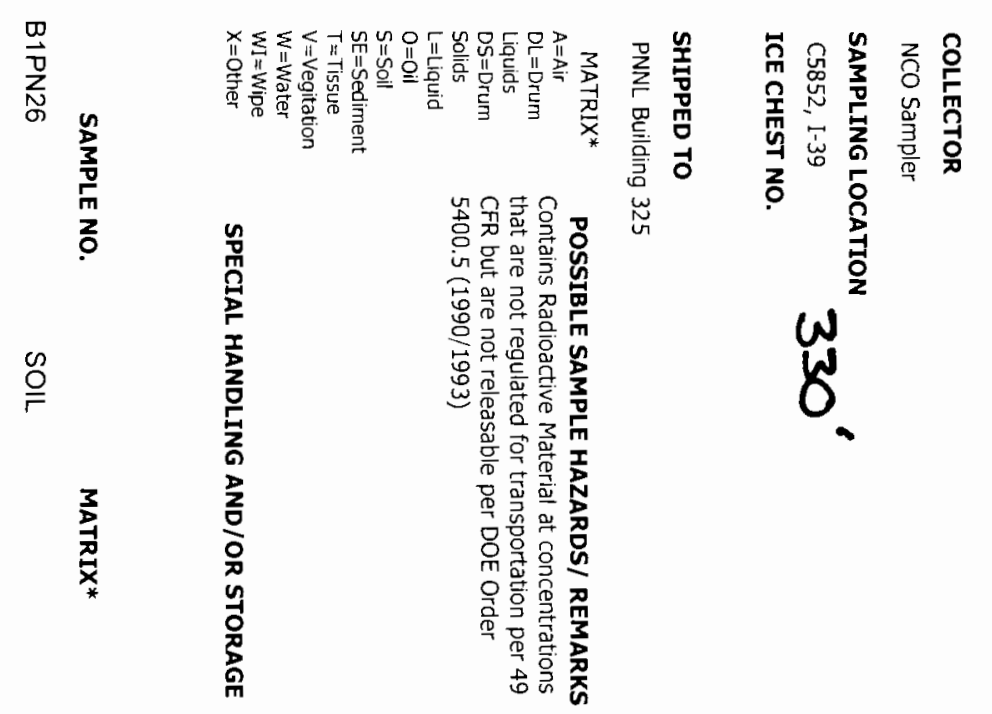

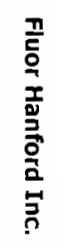

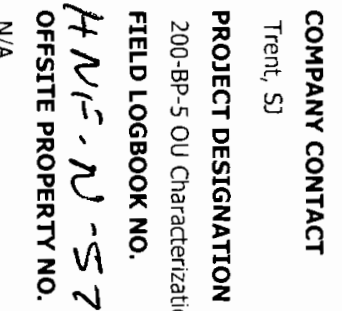
u $\overrightarrow{0}$

N

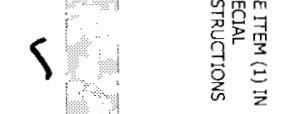

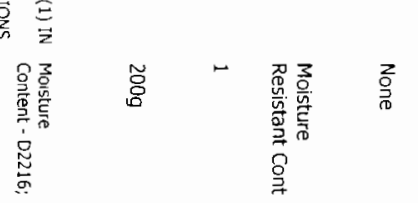

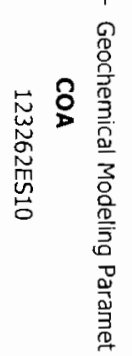
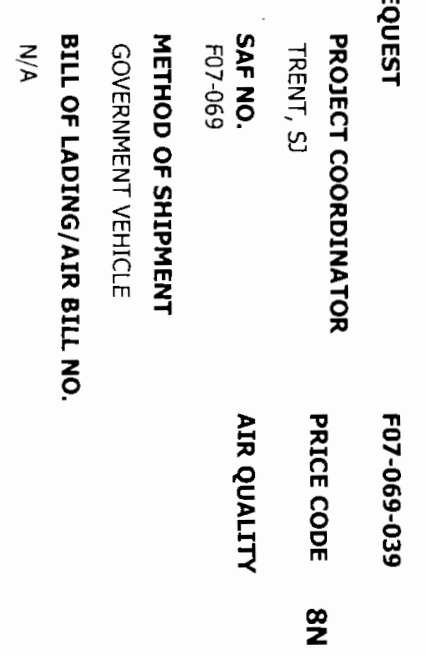


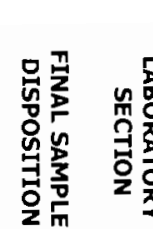
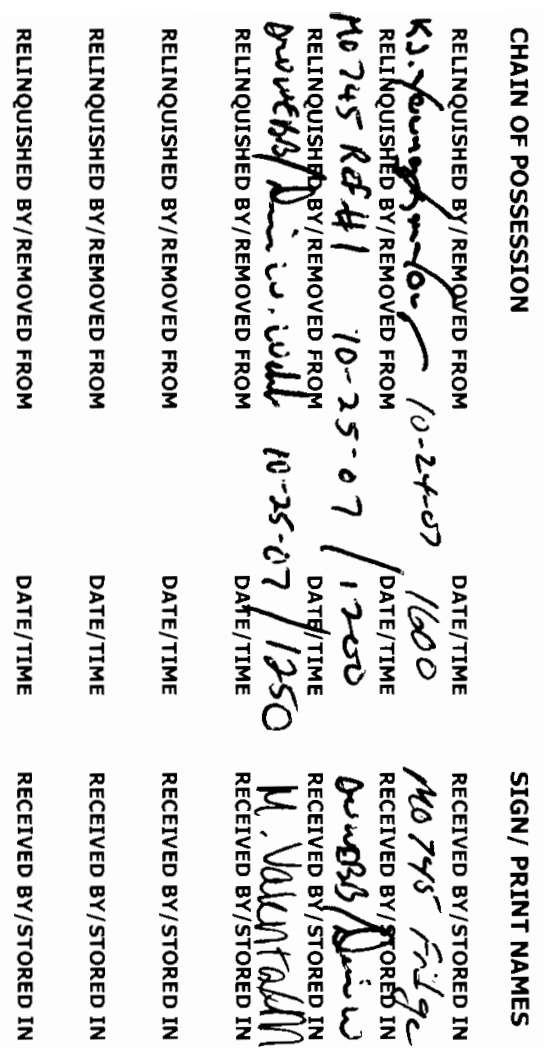

$\varepsilon \xi$

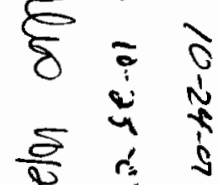

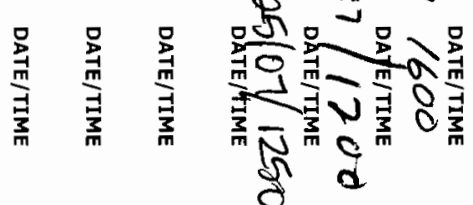

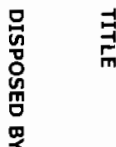

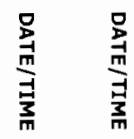

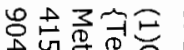

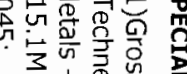

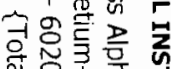

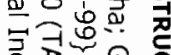

过起象高

言. 㟧言罗

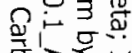

至宫它

으을

录突

茄品蛋

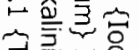

월유.

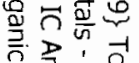

ญิ

它它畐

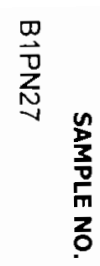

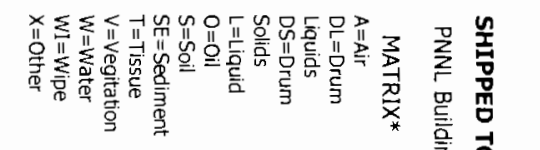
产

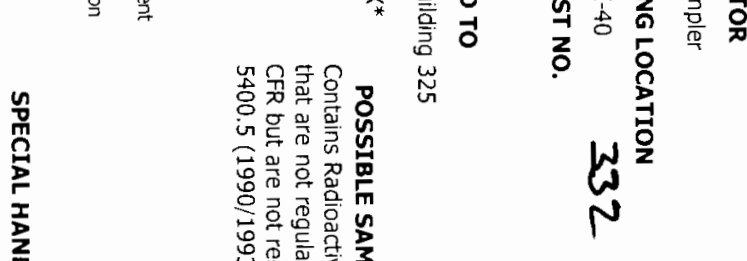

$\stackrel{0}{\stackrel{0}{ }}$

递 $\bar{\Phi}$

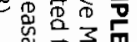

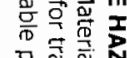

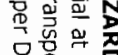

品实

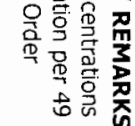

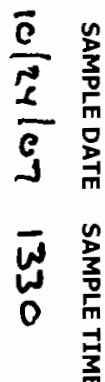

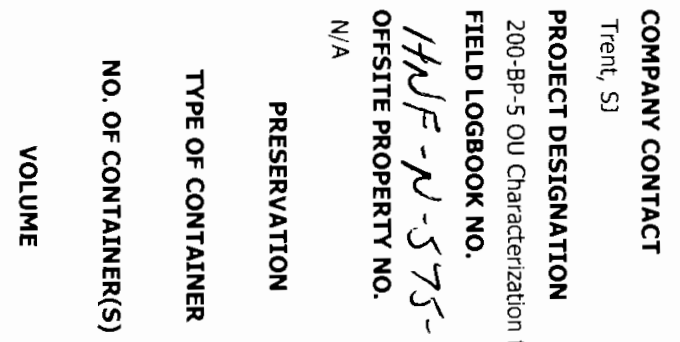

SU⿻

$+-\frac{2}{2} \frac{0}{0}$

\& $\overrightarrow{0}$

言资要

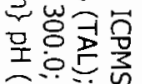

응

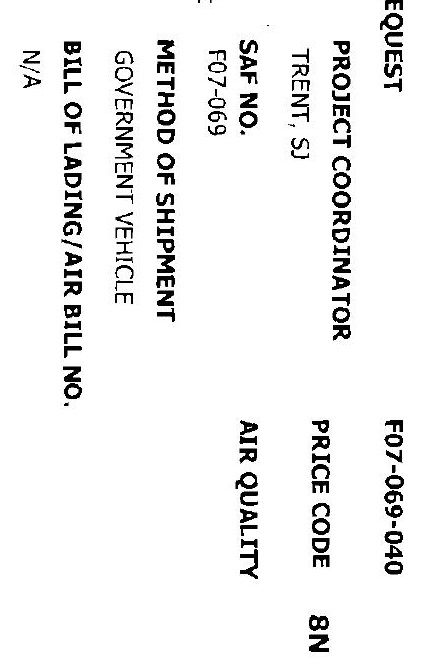

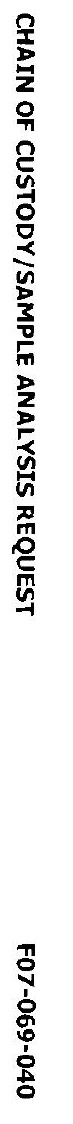




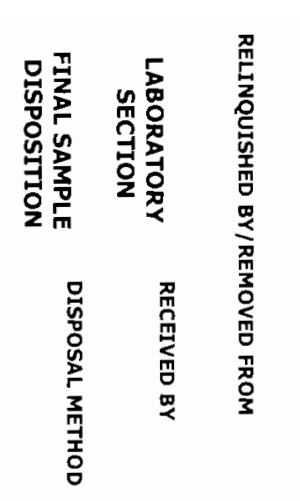
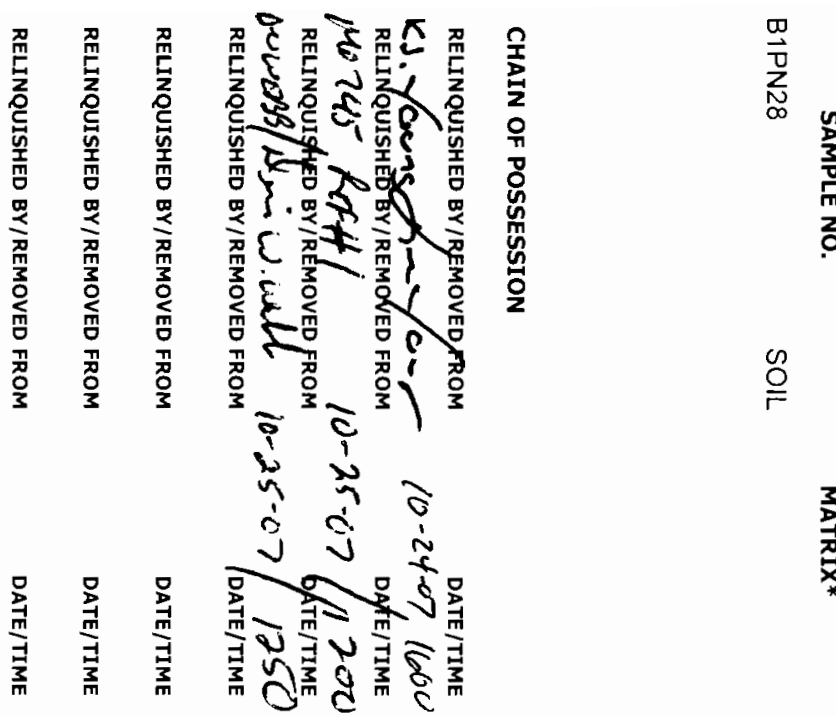

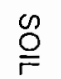
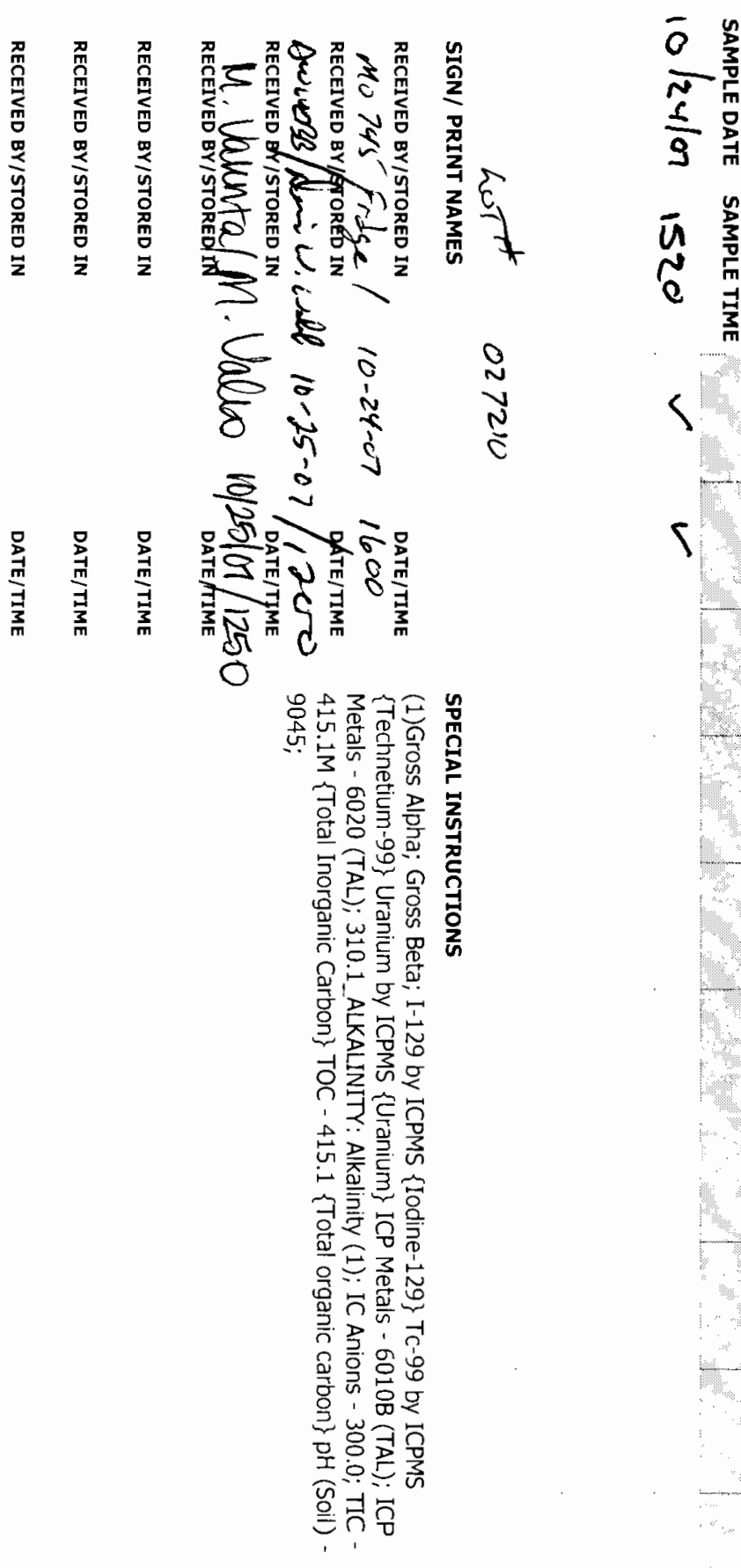

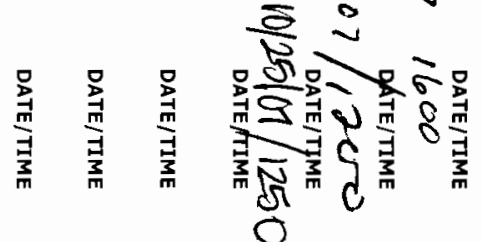

$\frac{3}{7}$

$\begin{array}{ll}\frac{9}{2} & \frac{2}{2} \\ \frac{m}{m} & \frac{m}{3} \\ \frac{7}{3} & \frac{1}{3} \\ \frac{m}{m} & \frac{m}{m}\end{array}$

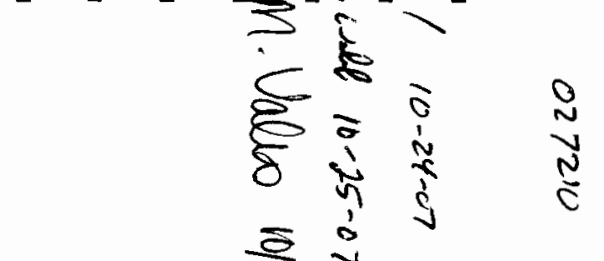

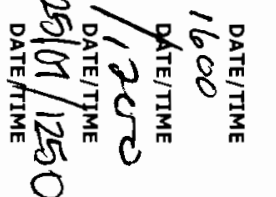

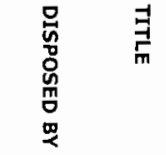

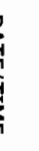

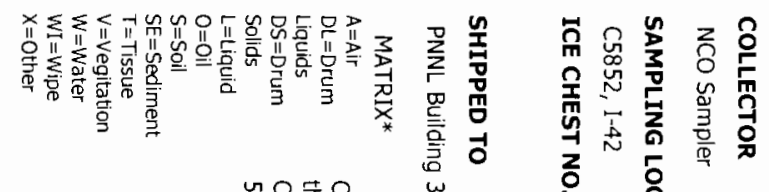

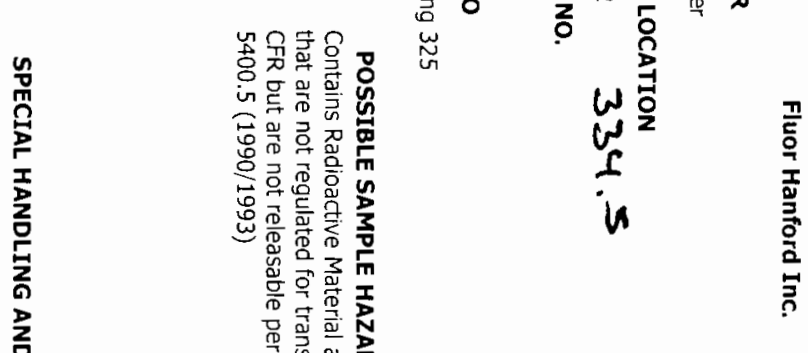

$\underset{*}{\stackrel{3}{2}}$

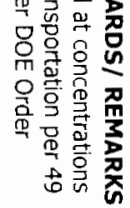

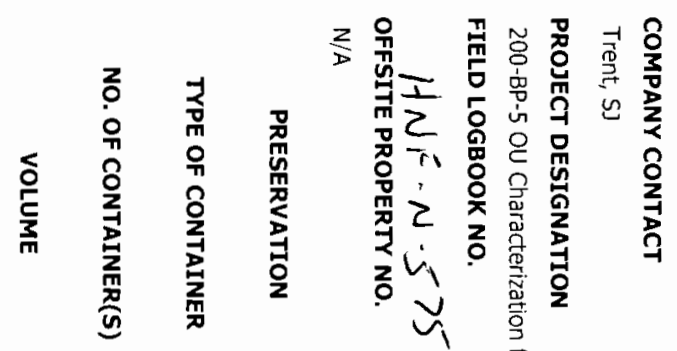

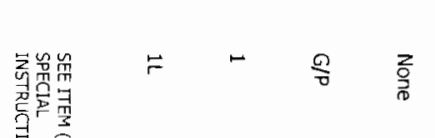

总芯

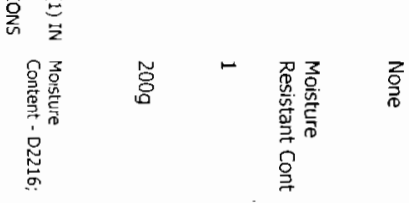




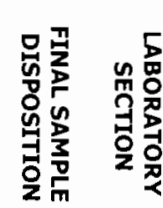

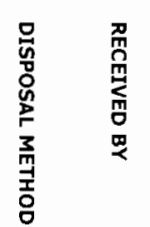

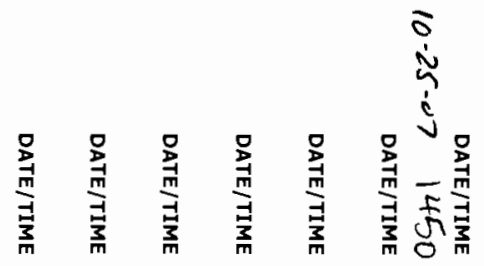
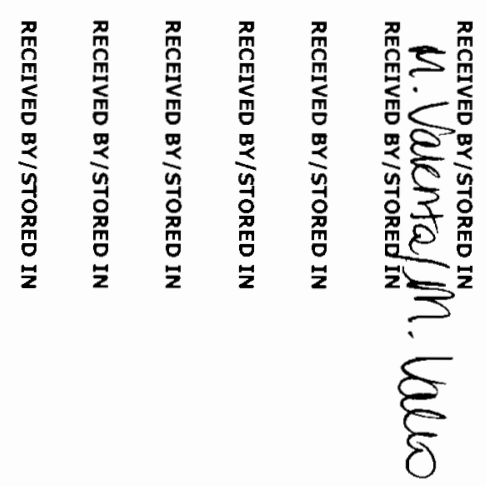

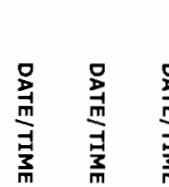

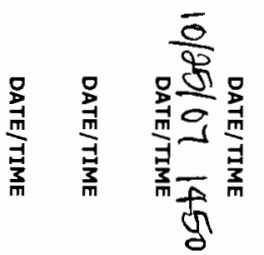

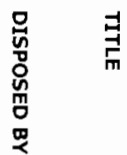

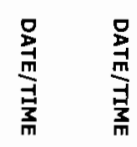

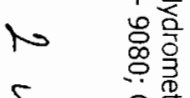

乞

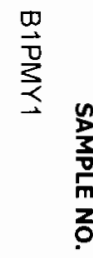

$\stackrel{\text { O }}{\rightleftharpoons}$

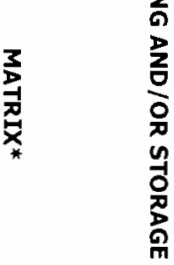

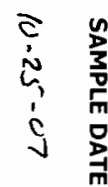

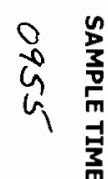

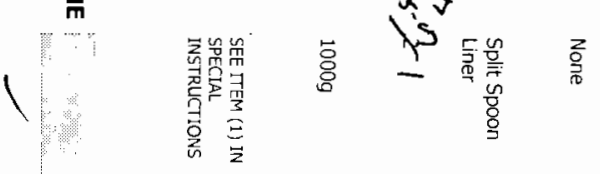

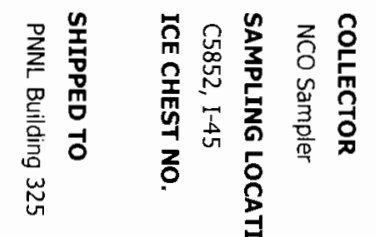
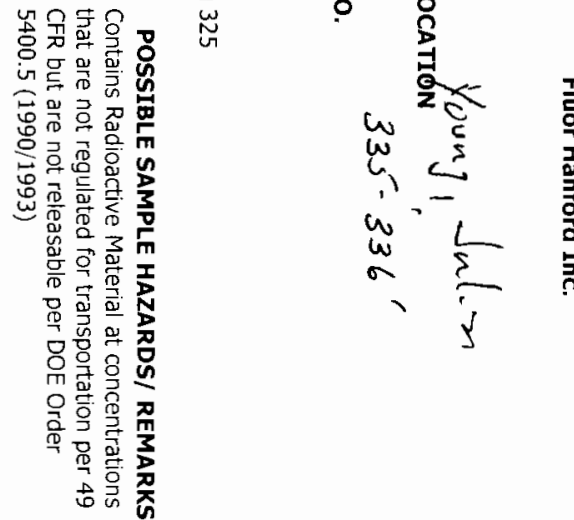

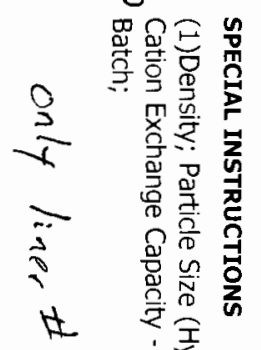

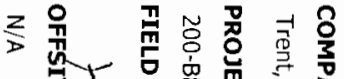

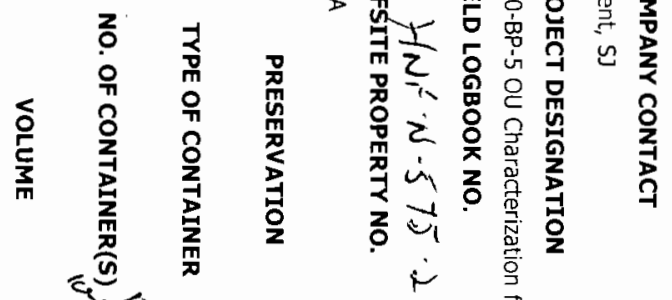

㞼

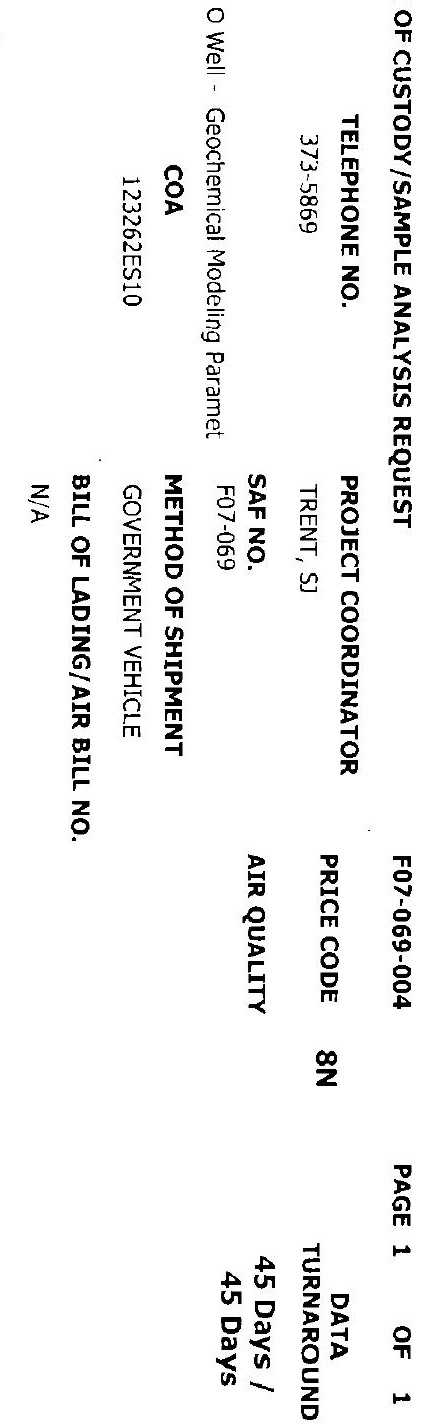




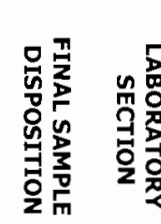

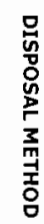
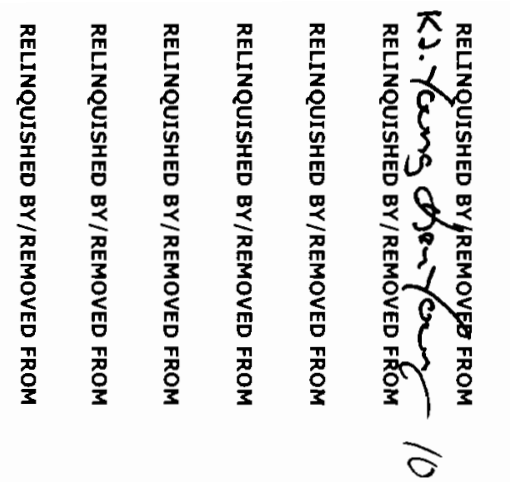

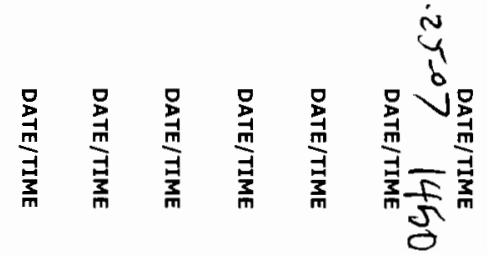

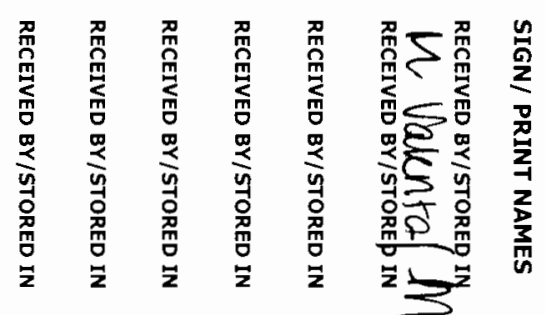

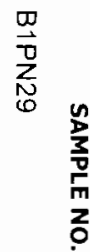

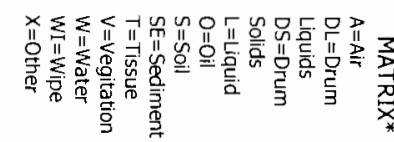

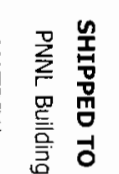

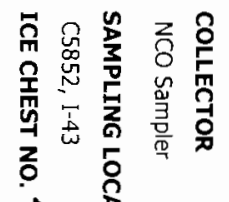

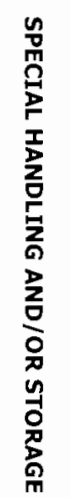

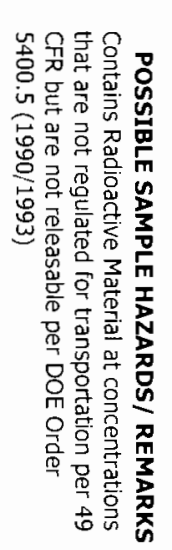

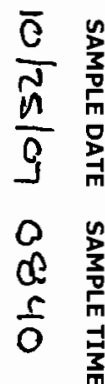

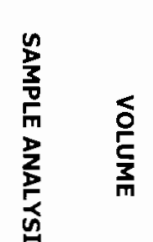

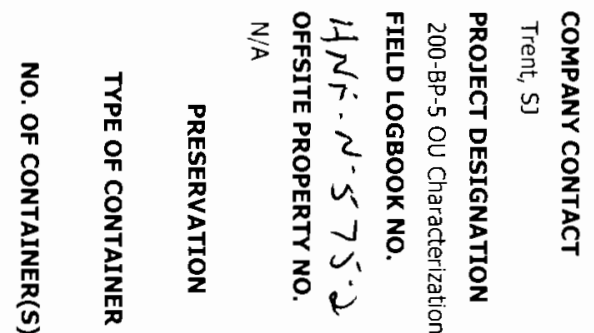

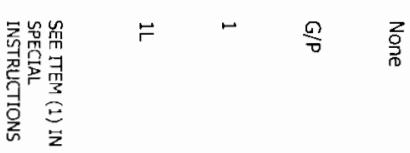

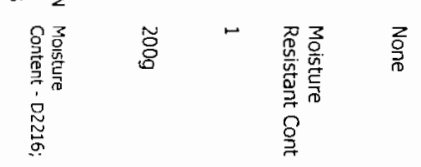

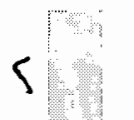

$\stackrel{\rho}{\circ}$

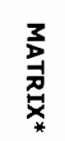
ज

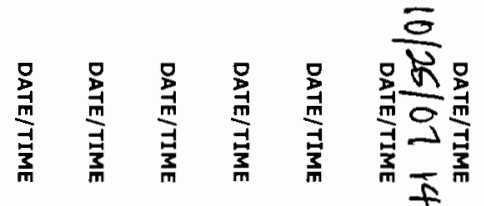

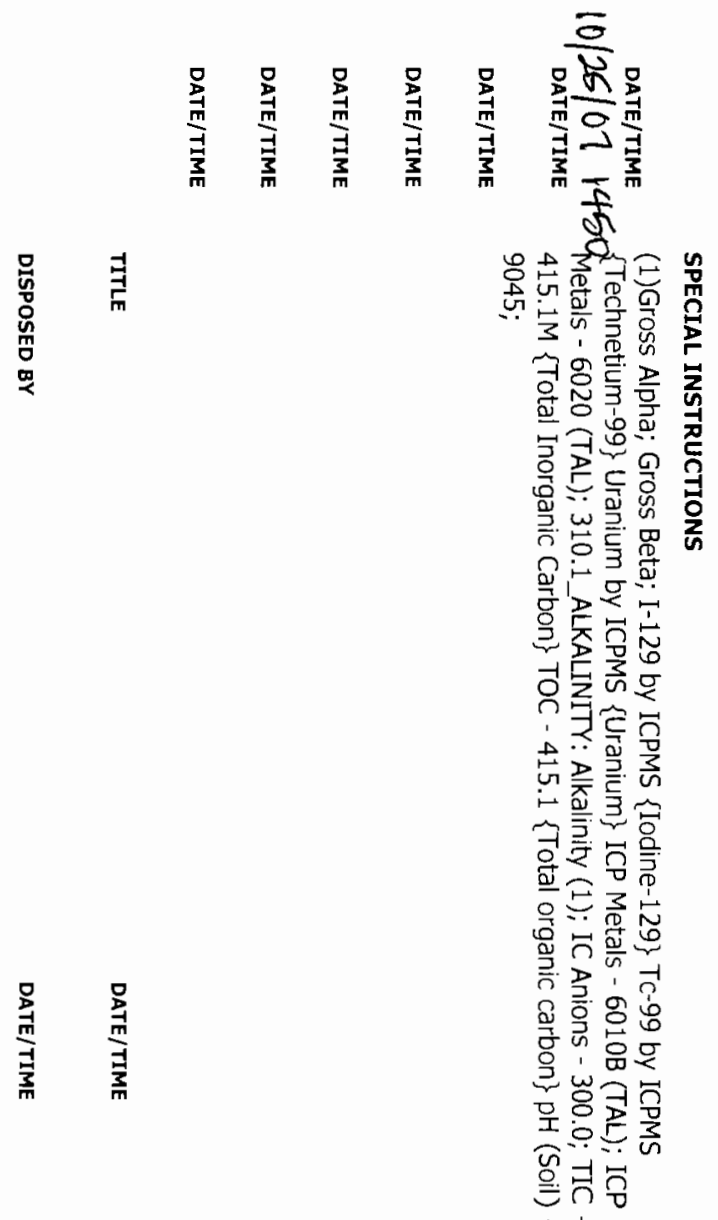

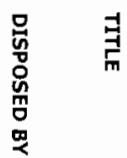

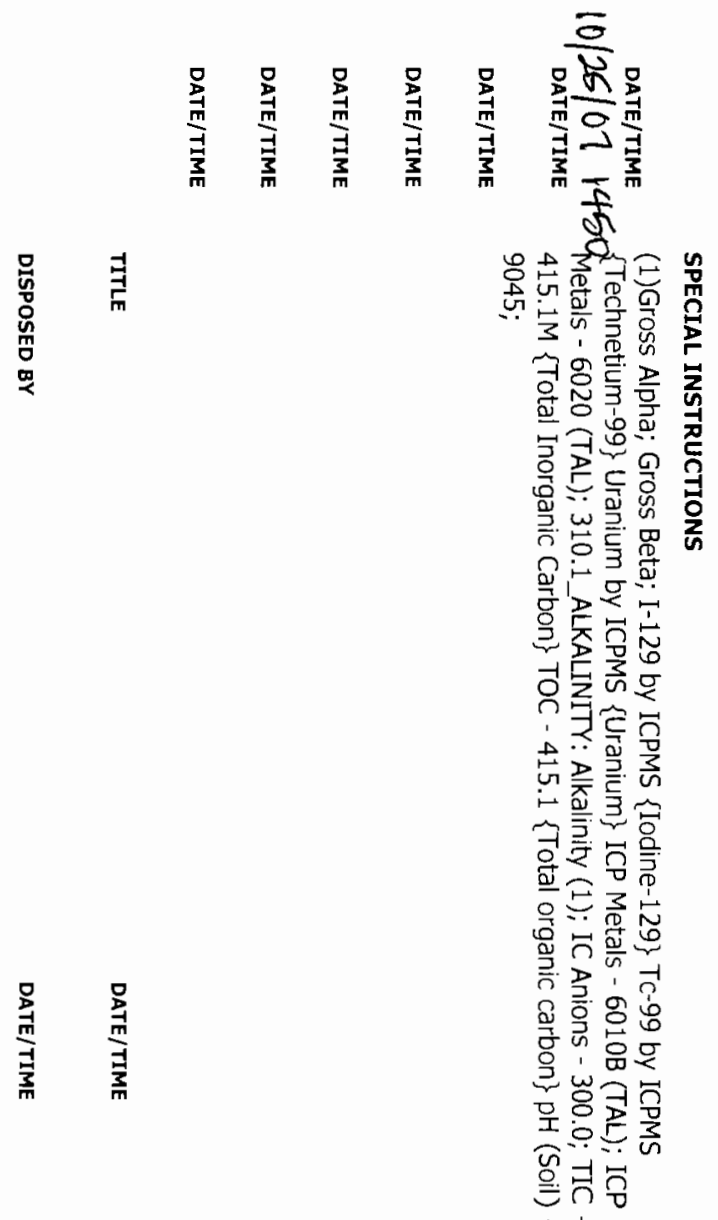

है

s

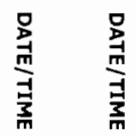




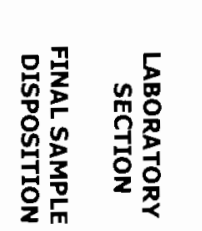

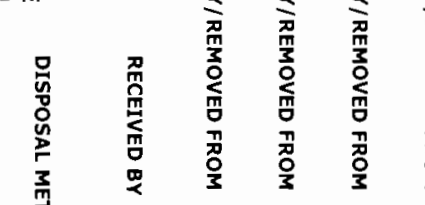
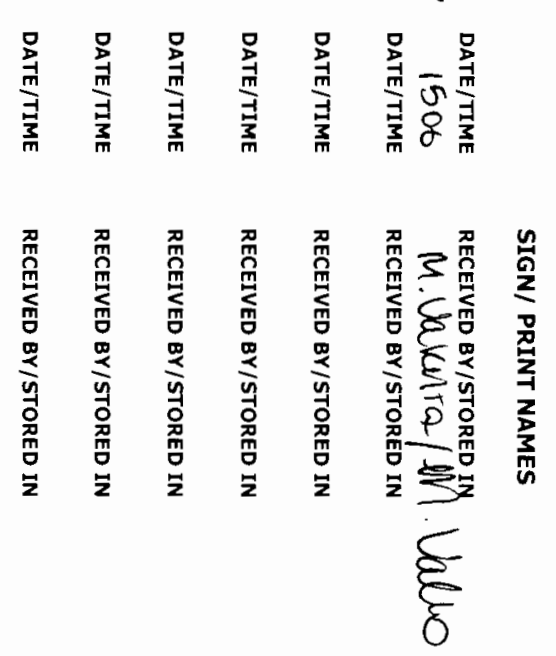

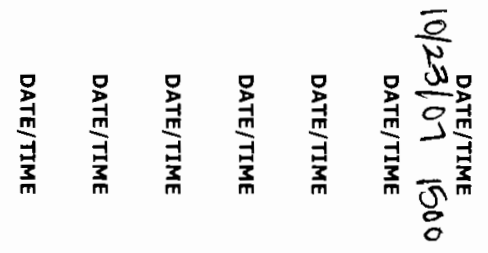

善

咅

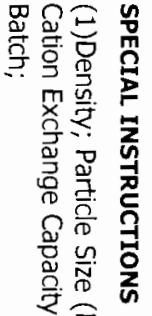

웅

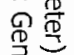

号家

需

管

设

폰

윽

등

용

응

点主

증 识
ழํํํ
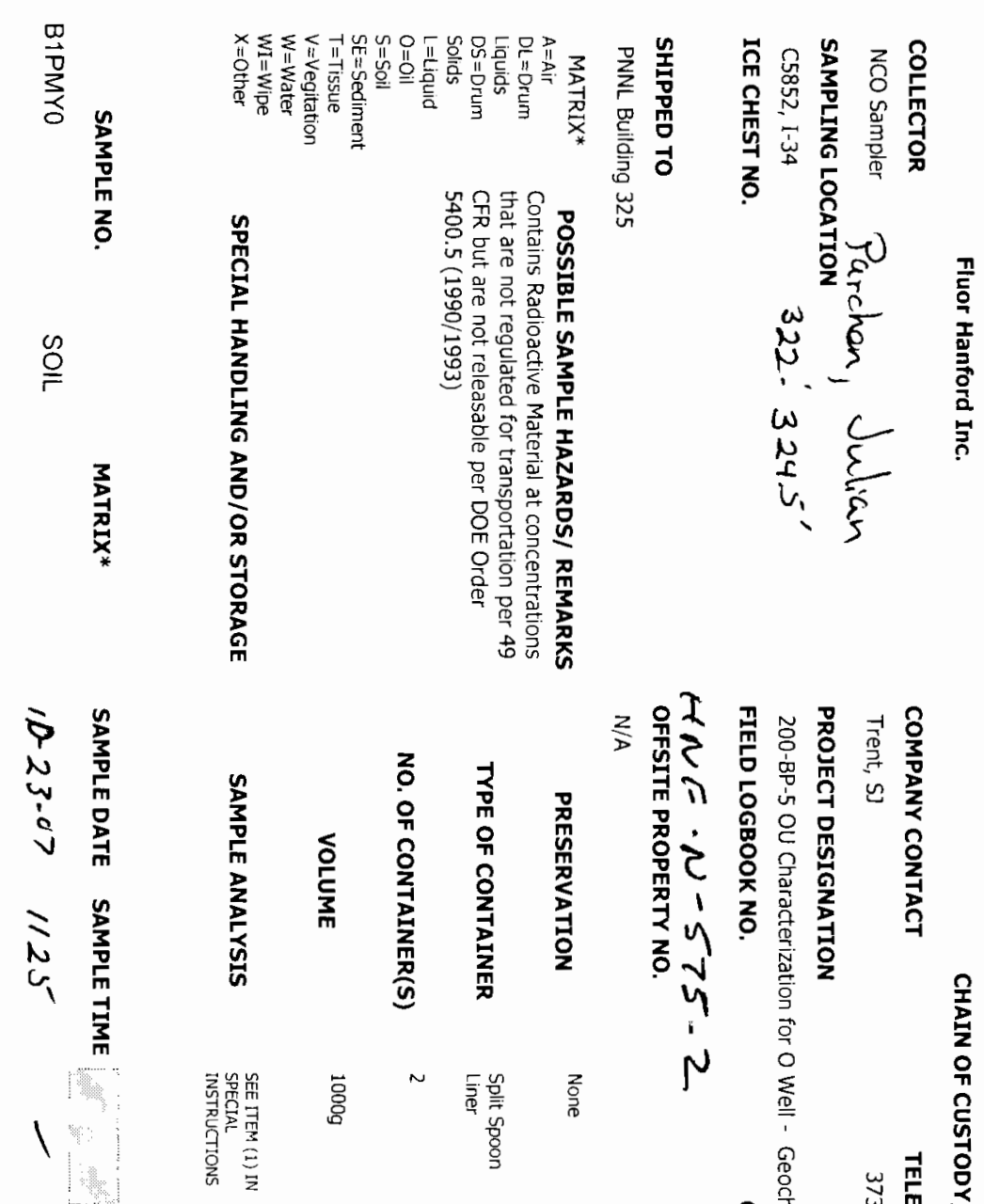

눈

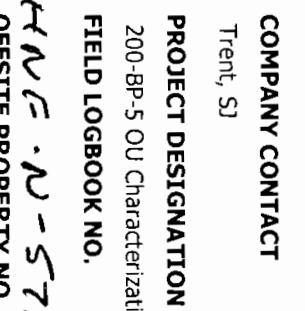




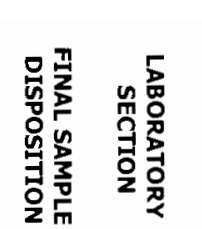

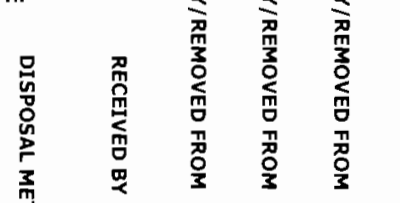

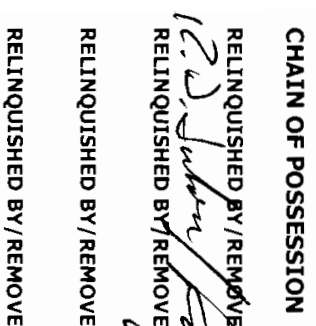

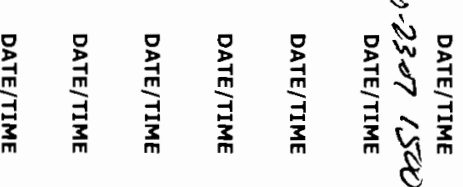
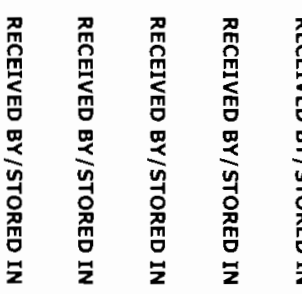

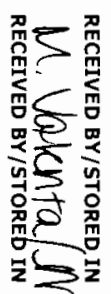

$\sum_{i} \quad 0$

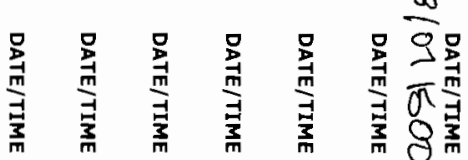

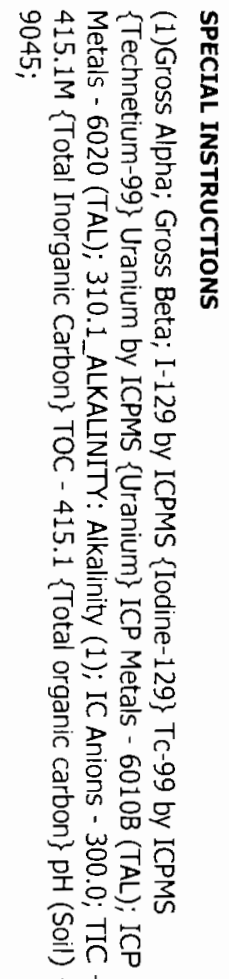

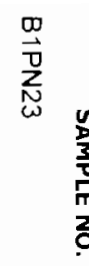

$\stackrel{\mathscr{O}}{\rightleftharpoons}$

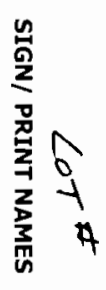

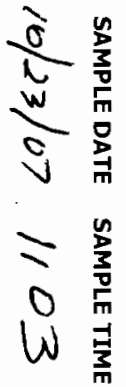

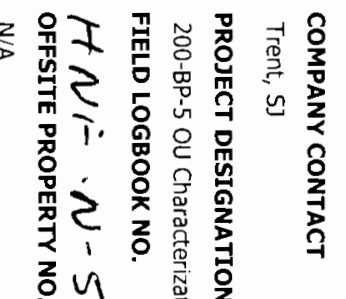

C.

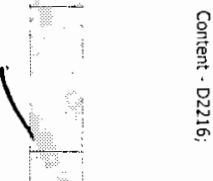

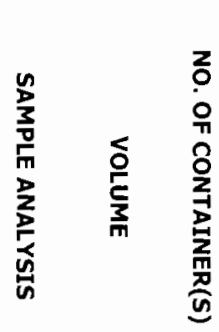

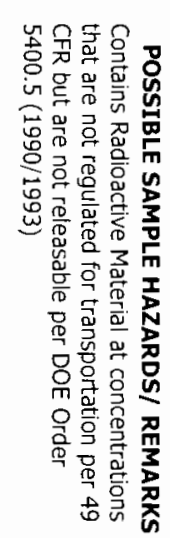

言

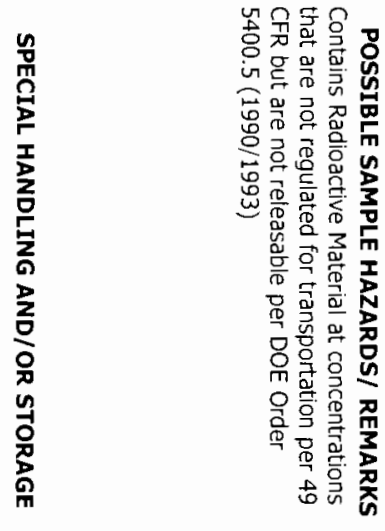

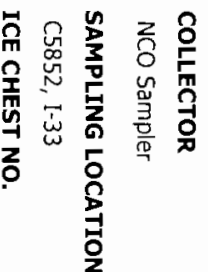

$\dot{w}$
$v$

$\frac{\pi}{\bar{E}}$

․

i

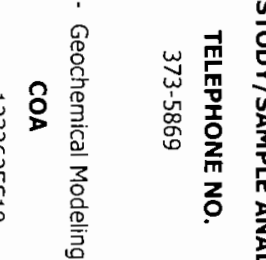

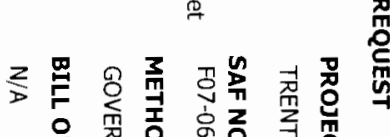

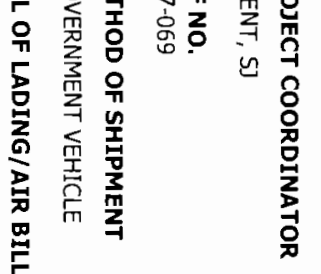

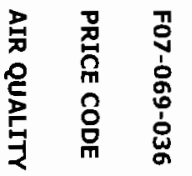

$\stackrel{\infty}{2}$

疍易

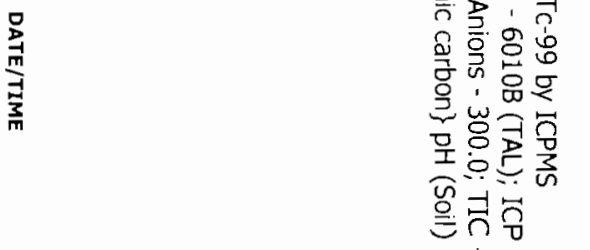




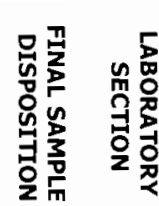

量亭

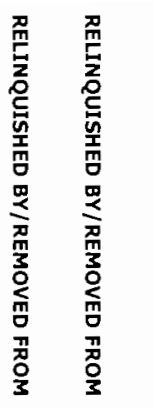

$I$

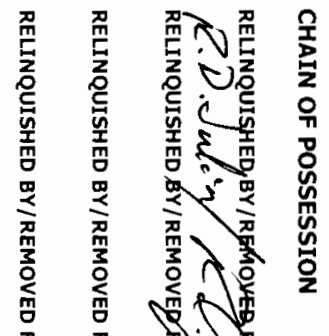

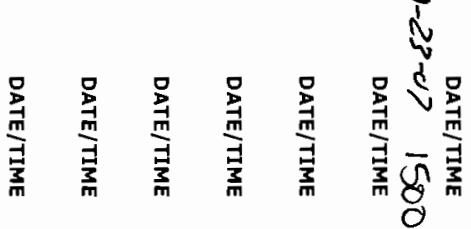

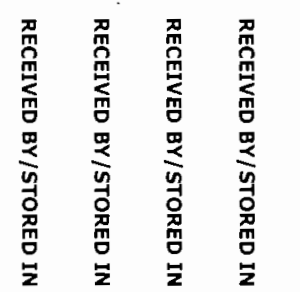

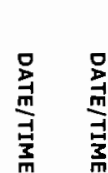

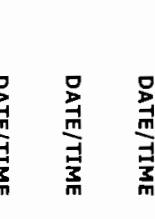

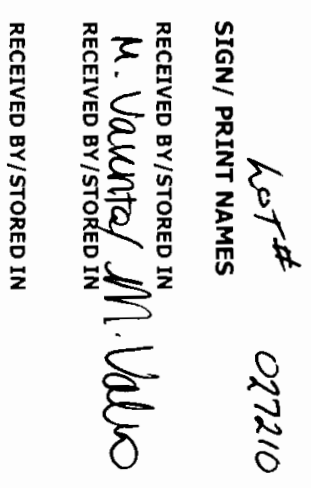

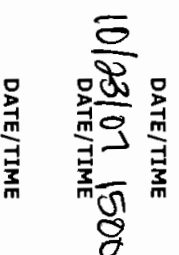

品 豆

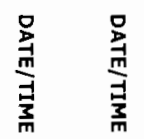

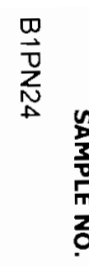

$\stackrel{\mathscr{\rho}}{=}$
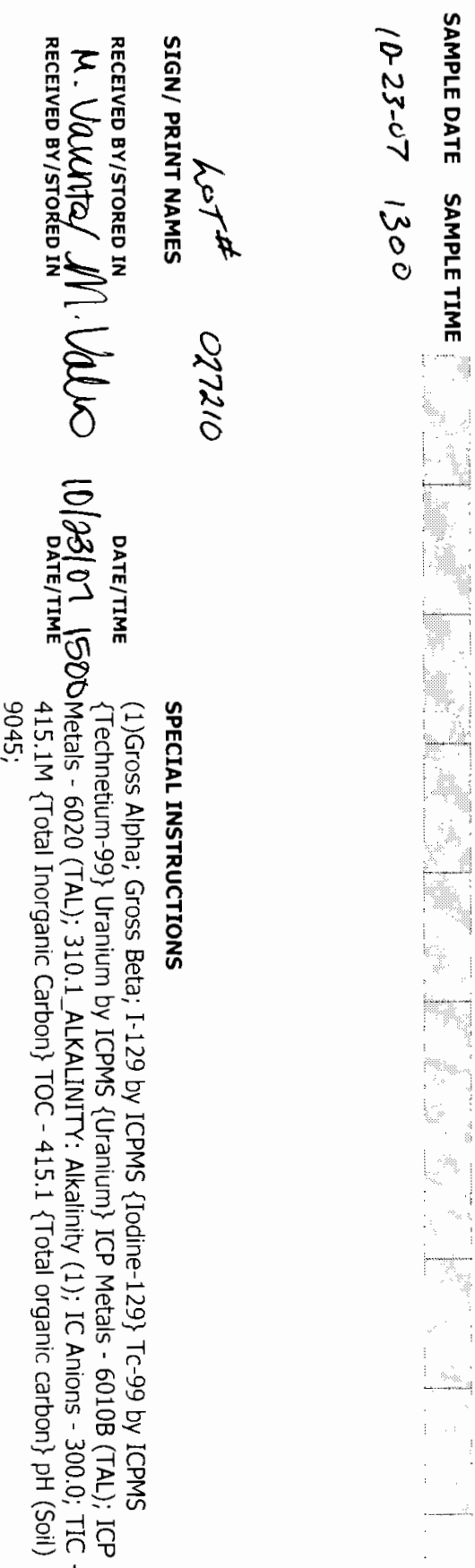

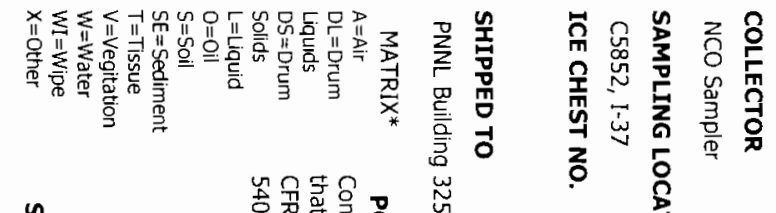
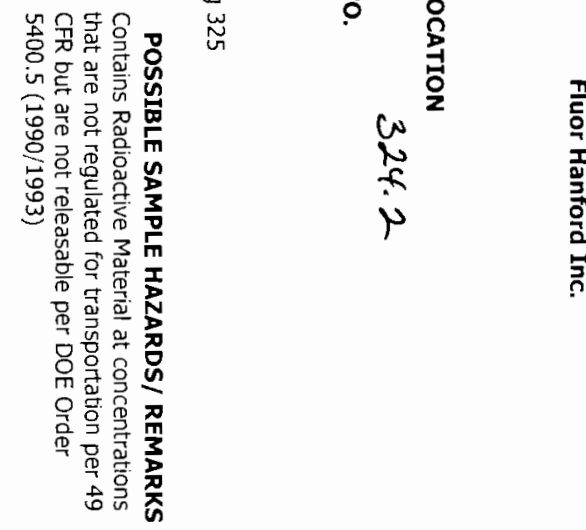

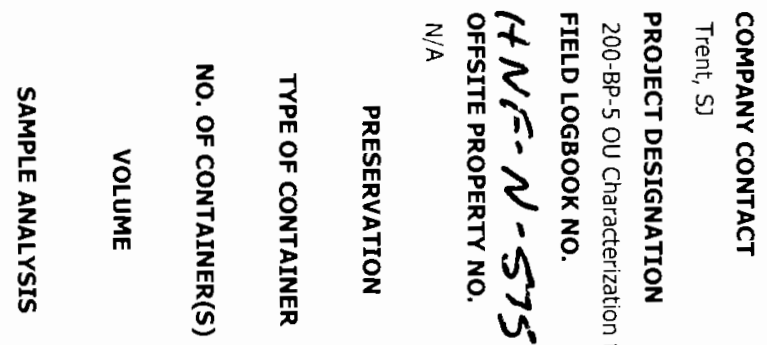

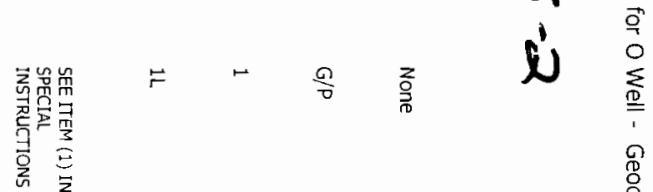

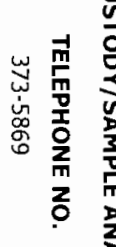

올

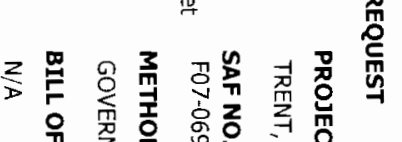

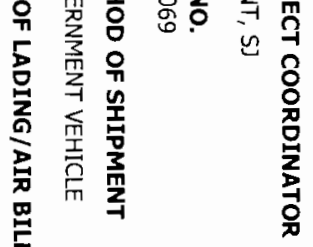

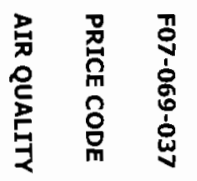

悉

高 


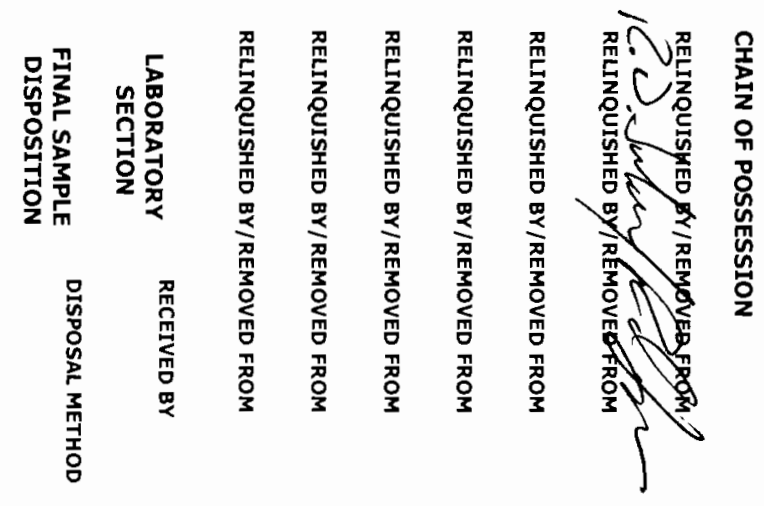

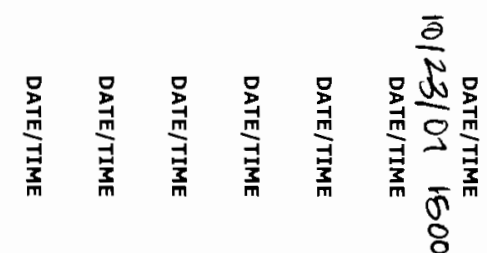

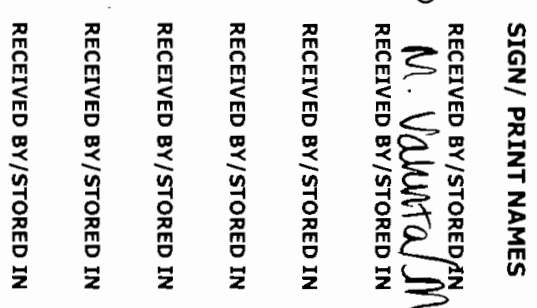

है

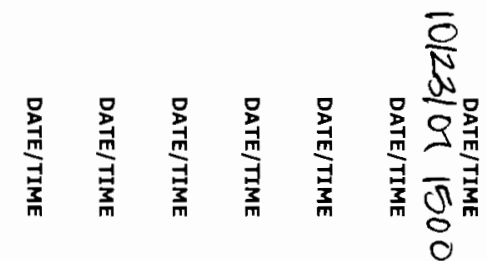

春

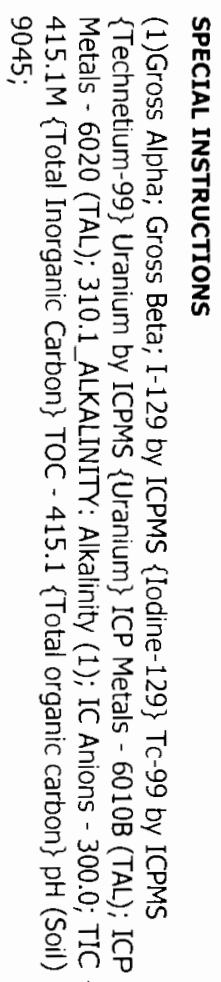

品是
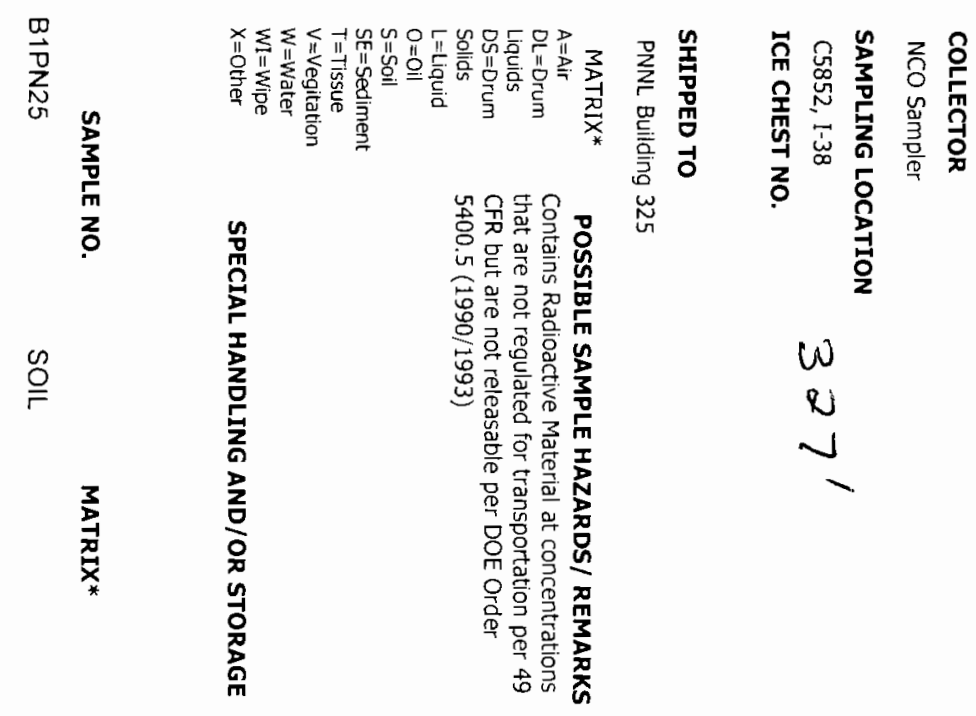

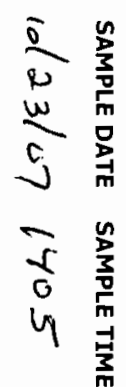

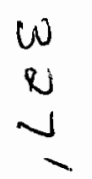

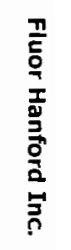
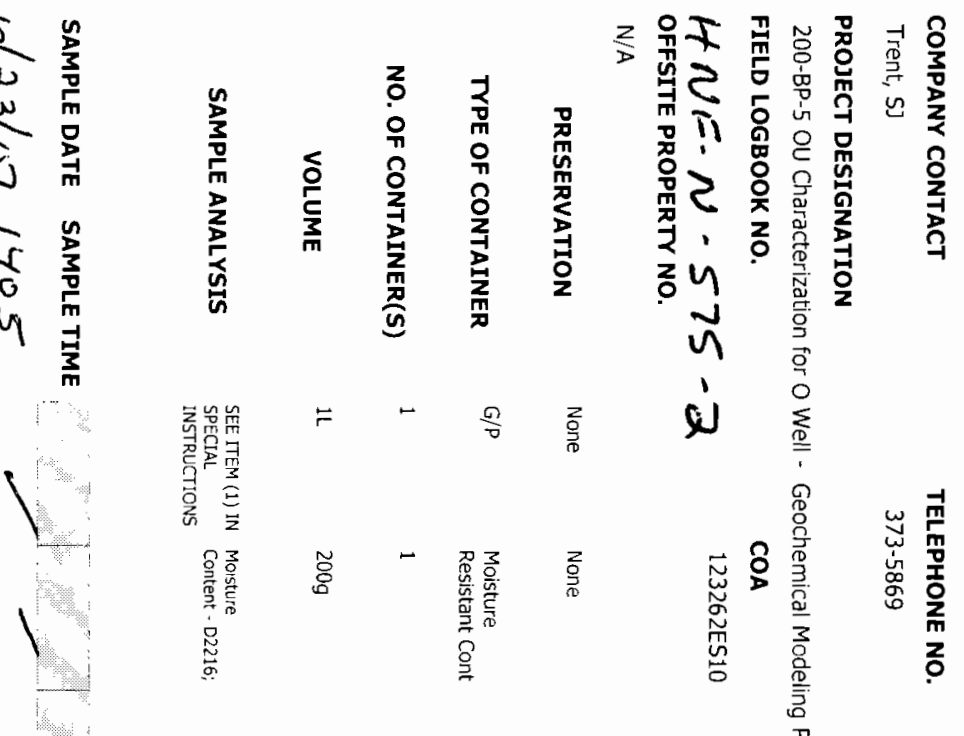

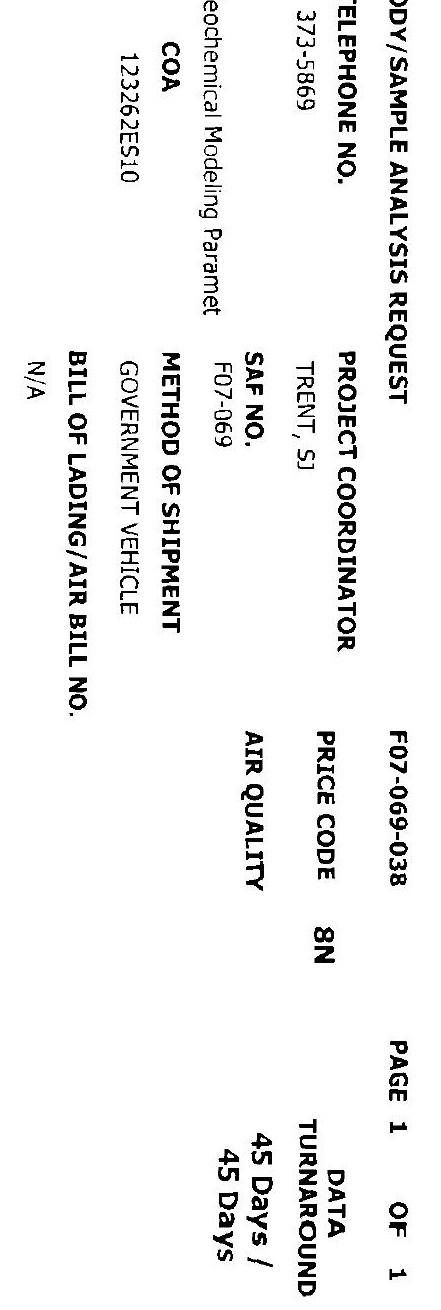

